\title{
WELL-CONSTRUCTION, WATER-QUALITY, AND WATER-LEVEL DATA, AND POND-INFILTRATION ESTIMATES, FOR THREE GROUND-WATER SUBBASINS, RIVERSIDE COUNTY, CALIFORNIA
}

By Carmen A. Burton, Charles A. Kaehler, and Allen H. Christensen

U.S. GEOLOGICAL SURVEY

Water-Resources Investigations Report 96-4294

Prepared in cooperation with the

EASTERN MUNICIPAL WATER DISTRICT

ஸे
g̊
유 


\section{U.S. DEPARTMENT OF THE INTERIOR BRUCE BABBITT, Secretary}

\section{U.S. GEOLOGICAL SURVEY \\ Gordon P. Eaton, Director}

Any use of trade, product, or firm names in this publication is for descriptive purposes only and does not imply endorsement by the U.S. Government.

For sale by the

U.S. Geological Survey

Information Services

Box 25286

Denver Federal Center

Denver, CO 80225

For additional information write to:

District Chief

U.S. Geological Survey

Placer Hall

6000 J Street

Sacramento, CA 95819-6129 


\section{CONTENTS}

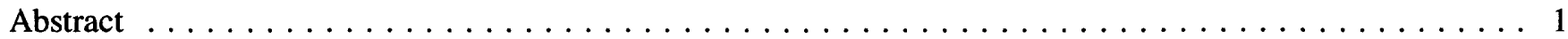

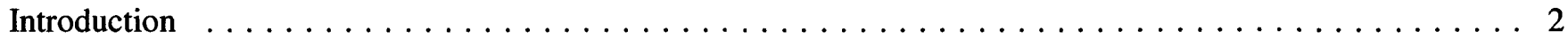

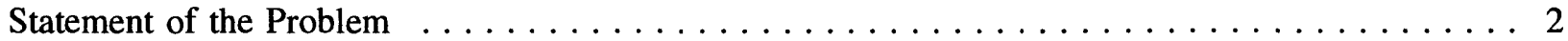

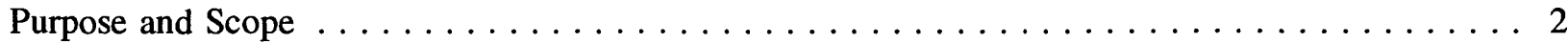

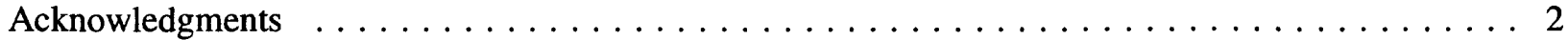

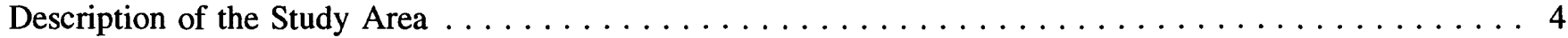

Existing Well-Construction, Water-Quality, and Water-Level Data $\ldots \ldots \ldots \ldots \ldots \ldots \ldots \ldots \ldots$

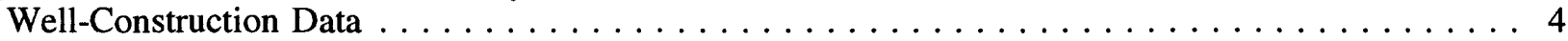

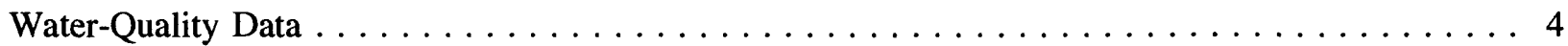

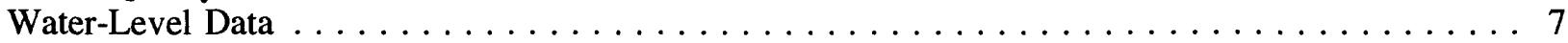

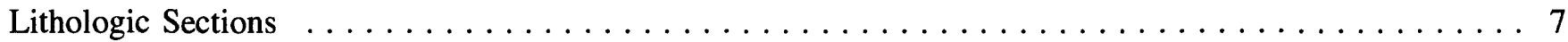

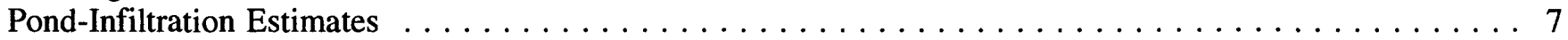

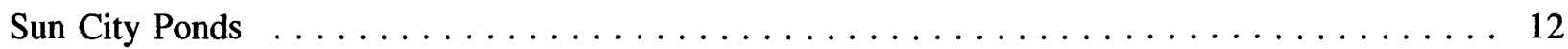

Trumble Road Pond $\ldots \ldots \ldots \ldots \ldots \ldots \ldots \ldots \ldots \ldots \ldots \ldots \ldots \ldots$

Winchester Ponds . . . . . . . . . . . . . . . . . . . . . . . . 20

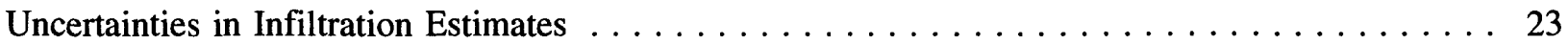

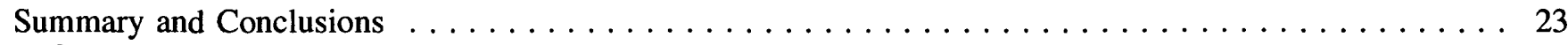

References Cited ... . . . . . . . . . . . . . . . . . . . . . . . . . . . . . . 24

\section{FIGURES}

1-4. Maps showing:

1. Location of study area and selected wells in the Winchester, Menifee, and south Perris

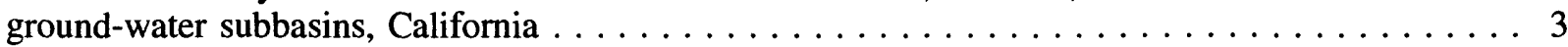

2. Water levels in the Winchester, Menifee, and south Perris ground-water subbasins, California, March 31 to June $6,1995 \ldots \ldots \ldots \ldots \ldots \ldots \ldots \ldots$

3. Water levels in the Winchester, Menifee, and south Perris ground-water subbasins, California,

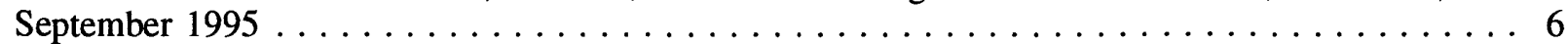

4. Location of lithologic sections $A-A^{\prime}, B-B^{\prime}$, and $C-C^{\prime}$ in the Winchester, Menifee, and south Perris ground-water subbasins, California

5-7. Lithologic sections of the Winchester, Menifee, and south Perris ground-water subbasins, California:

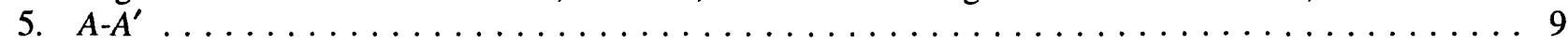

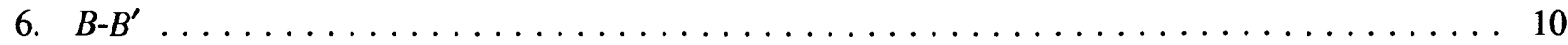

7. $C-C^{\prime} \ldots \ldots \ldots \ldots \ldots \ldots \ldots \ldots \ldots \ldots \ldots \ldots \ldots \ldots \ldots \ldots$

8. Maps showing pond sites, ground-water-level changes, and location of observation wells and

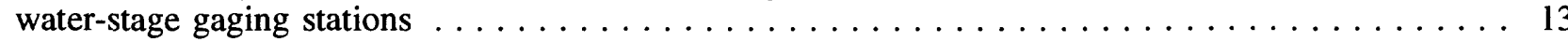

9. Graph showing water-level altitudes in Sun City pond no. 9, August 3 to September 15, 1995 . . . 14

10-11. Maps showing water-level altitudes at Sun City, Trumble Road, and Winchester sites:

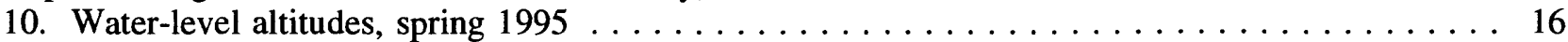

11. Water-level altitudes, autumn $1995 \ldots \ldots \ldots \ldots \ldots \ldots \ldots \ldots \ldots \ldots$

12-16. Graphs showing water-level altitudes in:

12. Sun City pond no. 9 for three 5 -day periods $\ldots \ldots \ldots \ldots \ldots \ldots \ldots \ldots \ldots \ldots \ldots$

13. Trumble Road pond, July 22 to September $27,1995 \ldots \ldots \ldots \ldots \ldots \ldots \ldots \ldots$

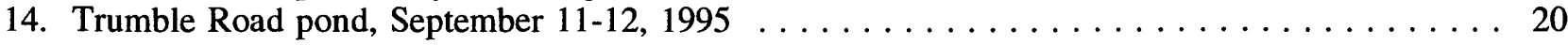

15. Winchester pond B, June 21 to September $6,1995 \ldots \ldots \ldots \ldots \ldots \ldots \ldots \ldots \ldots \ldots \ldots$

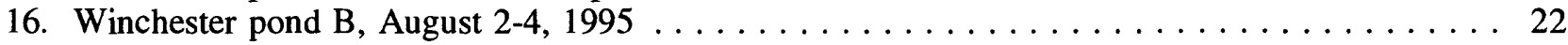




\section{TABLES}

1. Well-construction data for selected existing wells in the Winchester, Menifee, and south Perris

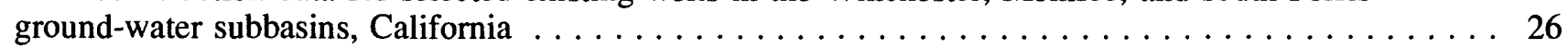

2. Historical water-quality data for selected wells in the Winchester, Menifee, and south Perris

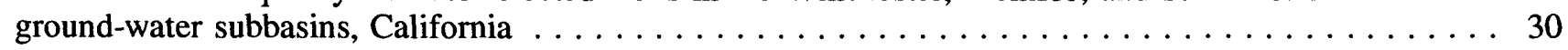

3. Water-level data for selected wells in the Winchester, Menifee, and south Perris gound-water

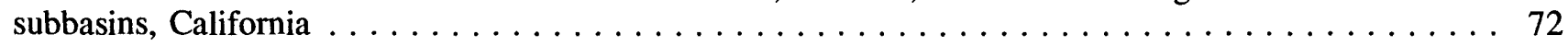

4. Water-level altitudes for Sun City storage pond no. 9, August-September $1995 \ldots \ldots \ldots$. . . . . . 90

5. Water-level altitudes for Sun City and Winchester observation wells, April-September 1995 . . . . . 96

6. Conversion of water-level altitude to pond volume for Pond No. 9, Sun City Regional Water

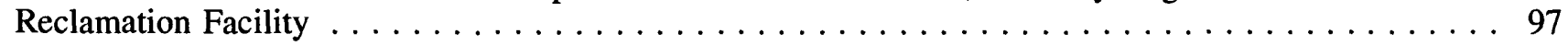

7. Daily timing of peak water levels for Sun City pond no. 9 and Trumble Road pond for selected periods, August-September $1995 \ldots \ldots \ldots \ldots \ldots \ldots \ldots \ldots \ldots \ldots \ldots \ldots \ldots$

8. Water-level altitudes for Trumble Road pond, July-September $1995 \ldots \ldots \ldots$. . . . . . . . . . . 99

9. Water-level altitudes for Winchester pond B (west pond), June-September $1995 \ldots \ldots \ldots 7$

\section{CONVERSION FACTORS, VERTICAL DATUM, WATER-QUALITY INFORMATION, ABBREVIATIONS, AND WELL-NUMBERING SYSTEM}

\section{Conversion Factors}

\begin{tabular}{rcl}
\hline Multiply & By & To obtain \\
\hline acre-foot (acre-ft) & 1,233 & cubic meter \\
acre-foot per year (acre-ft/yr) & 1,233 & cubic meter per year \\
acre-foot per day (acre-ft/d) & 1,233 & cubic meter per day \\
foot (ft) & 0.3048 & meter \\
gallon (gal) & 3.785 & liter (L) \\
inch (in.) & 2.54 & centimeter \\
inch (in.) & 25.4 & millimeter \\
mile (mi) & 1.609 & kilometer \\
square mile (mi $\left.{ }^{2}\right)$ & 2.590 & square kilometer \\
\hline
\end{tabular}

Temperature is given in degrees Celsius $\left({ }^{\circ} \mathrm{C}\right)$, which can be converted to degrees Fahrenheit $\left({ }^{\circ} \mathrm{F}\right)$ by the following equation:

$$
{ }^{\circ} \mathrm{F}=1.8\left({ }^{\circ} \mathrm{C}\right)+32 \text {. }
$$




\section{Vertical Datum}

Sea Level: In this report, "sea level" refers to the National Geodetic Vertical Datum of 1929—a geodetic datum derived from a general adjustment of the first-order level nets of the United States and Canada, formerly called Sea Level Datum of 1929.

\section{Water-Quality Information}

Chemical concentrations are given in milligrams per liter $(\mathrm{mg} / \mathrm{L})$ or micrograms per liter $(\mu \mathrm{g} / \mathrm{L})$. Milligrams per liter is approximately equivalent to parts per million; micrograms per liter is approximately equivalent to parts per billion.

\section{Abbreviations}

EMWD Eastern Municipal Water District

MWD Metropolitan Water District

USGS U.S. Geological Survey

RCFCD Riverside County Flood Control and Water Conservation District

RWRF Regional Water-Reclamation Facility

$\mu \mathrm{S} / \mathrm{cm} \quad$ microsiemen per centimeter at $25^{\circ} \mathrm{Celsius}$

$\mathrm{gal} / \mathrm{min} \quad$ gallons per minute

$\mathrm{gal} / \mathrm{d} \quad$ gallons per day

$\mathrm{ft} / \mathrm{d} \quad$ foot per day 


\section{Well-Numbering System}

Wells are identified and numbered according to their location in the rectangular system for the subdivision of public lands. Identification consists of the township number, north or south; the range number, east or west; and the section numbers. Each section is divided into sixteen 40 -acre tracts lettered consecutively (except I and $\mathrm{O}$ ), beginning with " $\mathrm{A}$ " in the northeast corner of the section and progressing in a sinusoidal manner to "R" in the southeast corner. Within the 40 -acre tract, wells are sequentially numbered in the order they are inventoried. The final letter refers to the base line and meridian. In California, there are three base lines and meridians; Humboldt (H), Mount Diablo (M), and San Bernardino (S). All wells in the study area are referenced to the San Bernardino base line and meridian (S). Well numbers consist of 15 characters and follow the format $005 \mathrm{~S} 003 \mathrm{~W} 32 \mathrm{~A} 001 \mathrm{~S}$. In this report, well numbers are abbreviated and written $5 \mathrm{~S} / 3 \mathrm{~W}-32 \mathrm{~A} 1$. Wells in the same township and range are referred to by only their section designation, 32A1. The following diagram shows how the number for well $5 \mathrm{~S} / 3 \mathrm{~W}-32 \mathrm{~A} 1$ is derived.

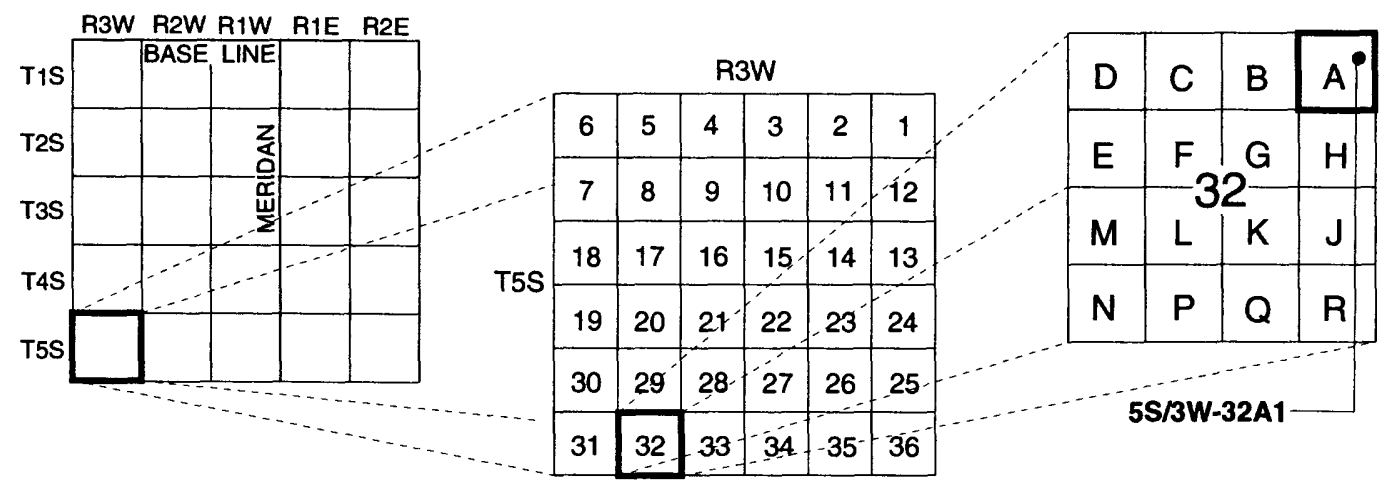




\title{
WELL-CONSTRUCTION, WATER-QUALITY, AND WATER-LEVEL DATA, AND POND-INFILTRATION ESTIMATES, FOR THREE GROUND-WATER SUBBASINS, RIVERSIDE COUNTY,
}

\section{CALIFORNIA}

\author{
By Carmen A. Burton, Charles A. Kaehler, and Allen H. Christensen
}

\begin{abstract}
Reclaimed water in the Eastern Municipal Water District of Riverside County, California, is used within the service area for agricultural irrigation. Owing to the seasonal demand for reclaimed water, storage/infiltration ponds were constructed in the Winchester, Menifee, and south Perris subbasins. Reclaimed water infiltrates from these ponds and enters the ground-water system. Little is known of the effects of the reclaimed water on ground-water quality. In cooperation with the Eastern Municipal Water District, the U.S. Geological Survey began a study in 1995 to determine the quantity and fate of reclaimed water percolating from these storage ponds. Data compiled during the first phase of this study are presented in this report.
\end{abstract}

Field reconnaissance of the Winchester, Menifee, and south Perris subbasins indicated the existence of many wells. Well-construction data for 115 of these wells were tabulated. Available historical water-quality and waterlevel data for 178 wells in the subbasins also were tabulated. In addition, water levels in 86 wells were measured during the spring and autumn of 1995. On the basis of these data, water-level contour lines were drawn and the direction of ground-water flow was determined. Three lithologic sections through the subbasins were constructed from drillers' logs of 26 wells.
Water-level data were collected from one pond at each of the three storage-pond sites. Water-level data and evaporation data, for periods of no pumped inflow or pumped outflow, were used to estimate an infiltration rate of -0.18 to -0.15 acre-feet/day (upward percolation) for Sun City pond no. 9 during August 3-September 15, 1995, and a rate of 0.72 acre-feet/day for Trumble Road pond during September 11-12, 1995. For Winchester pond $B$, a rate of -5.35 acre-feet/day was estimated for July 28-August 19, 1995, during which time pumped inflow and outflow were measured. The Trumble Road pond estimate was made on the basis of nighttime measurements and assumes negligible evaporation.

The upward percolation of water in Sun City pond no. 9 and Winchester pond B probably resulted from the pumped lowering of the water level in the ponds to an altitude below the local water-table altitude, followed by a gradual rise as the pond level equilibrated with the water-table altitude. The range of values and direction of infiltration may be indicative both of the variability of infiltration rates at different sites (and under different conditions) and the possible error ranges of the measured parameters used in calculating infiltration. At any of the sites, infiltration rates during times when the ponds are at high stage are likely to be different from the rates estimated using primarily low-stage data. 


\section{INTRODUCTION}

Artificial recharge of ground water with reclaimed water has been an increasingly important topic for study in recent years as the need for reuse of water has grown. Two common mechanisms of artificial recharge of ground water are percolation of reclaimed water from infiltration ponds and percolation of reclaimed water applied as irrigation.

Several studies have shown that the effects of artificial recharge with reclaimed water on ground-water conditions vary from site to site. In a study in the Livermore-Amador Valley in Alameda County, California, ground-water quality was found to be degraded in all areas where reclaimed water was applied (Sylvester, 1983). In an aquifer near Tallahassee, Florida, increases in nitrate and chloride concentrations were attributed to irrigation with reclaimed water (Pruitt and others, 1988). Surfactant-derived residues (that is, methylene blue activated substances) also have been detected in ground water affected by recharge areas (Field and others, 1992a,b). In contrast, results of a study at East Meadow, Long Island, New York, suggest that recharging with reclaimed water decreased concentrations of nitrate-nitrogen and several low-molecular-weight hydrocarbons, thereby improving ground-water quality (Schneider and others, 1987).

\section{Statement of the Problem}

The Eastern Municipal Water District (EMWD), located in southwestern Riverside County, California, serves a population of 350,000 in a service area that exceeds $550 \mathrm{mi}^{2}$. The EMWD provides both domestic and irrigation water and operates five regional water-reclamation facilities (RWRF's). In 1995, the five RWRF's had a combined reclaimed-water production of 31,900 acre$\mathrm{ft} / \mathrm{yr}$, all of which is used within the EMWD service area-mostly for agricultural irrigation or irrigation of golf courses. Because demand for the reclaimed water is seasonal, three storage/ infiltration-pond sites have been constructed: near the town of
Winchester; at the Sun City RWRF; and at the Perris RWRF (including the Trumble Road pond) (fig.1). It is estimated by EMWD that about 13,000 acre- $\mathrm{ft}$ of reclaimed water is lost annually, incidental to the operation of the storage ponds, as a result of evaporation and percolation to the ground-water system. The ponds near Winchester and at Sun City RWRF are located in subbasins in which the ground water is characterized by high dissolvedsolids concentration. The quality of reclaimed water percolating from those ponds is better than that of the ground water.

\section{Purpose and Scope}

In 1995, the U.S. Geological Survey (USGS), in cooperation with EMWD, began a study to determine the quantity and fate of water percolating from the reclaimed-water storage/infiltration ponds to the ground-water system. Although some of the ponds have been in operation for 33 years, little is known about the effects of recharge from the ponds on ground water. The purposes of this report are to present (1) data on historical water quality, historical and current water levels, and construction of existing wells; (2) borehole-lithologic descriptions and depth-to-bedrock data; and (3) pond water-level data and infiltration estimates. These data will be useful for future interpretative reports from this study. In addition, general directions of ground water flow in the three subbasins were determined, on the basis of waterlevel data collected during the study. Water-level contour maps are presented in this report.

\section{Acknowledgments}

The authors gratefully acknowledge the help of Jeffrey Hale, Alfred Javier, Stephen Crombie, and other EMWD personnel for their help in compiling the data presented in this report, for permitting use of EMWD equipment, and for providing access to EMWD facilities. The authors also acknowledge the many well owners for their cooperation in obtaining water-level measurements and collecting water samples. 


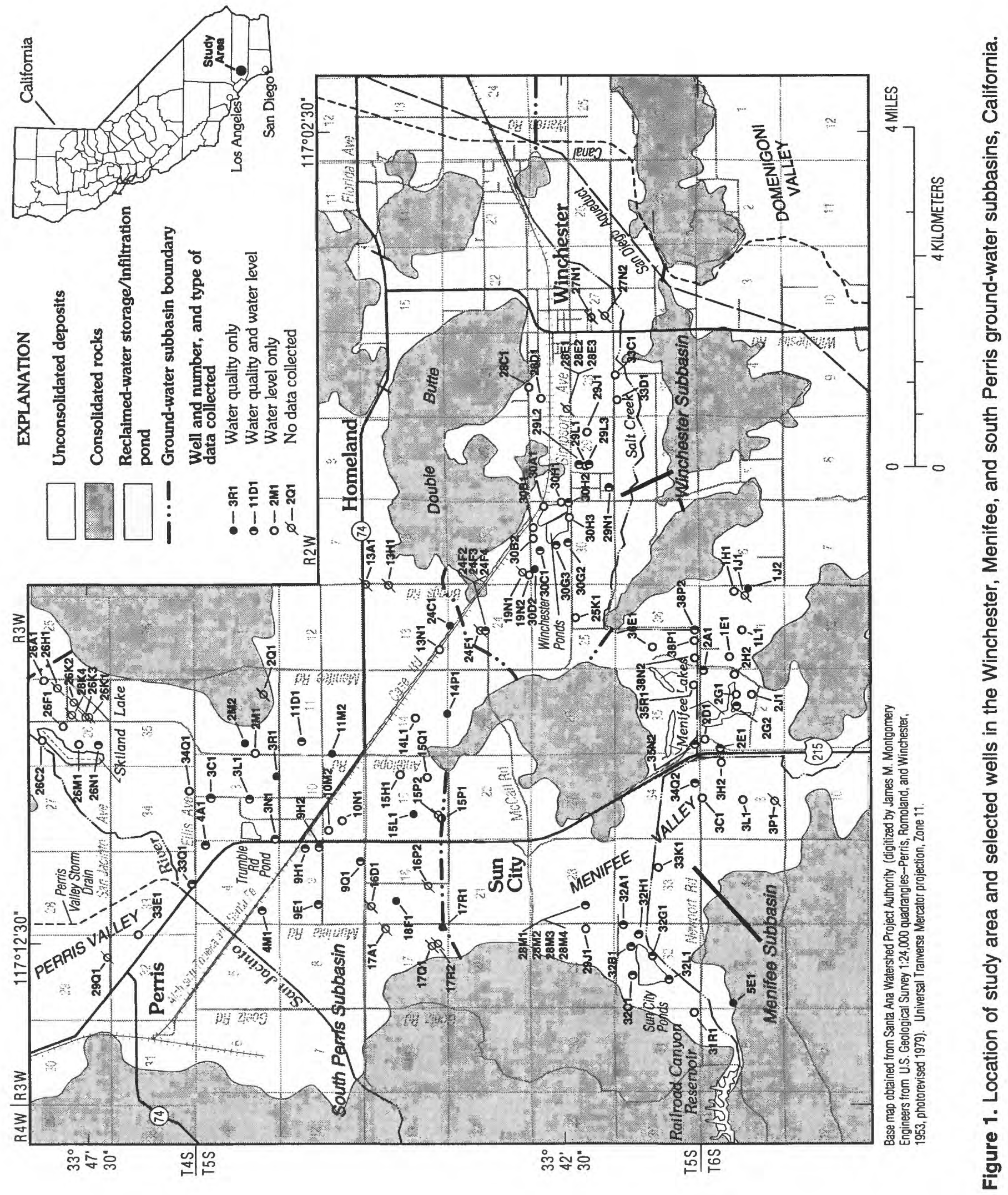




\section{DESCRIPTION OF THE STUDY AREA}

The study area includes all or part of the ground-water subbasins in which the three storage ponds are located: the Winchester subbasin, the Menifee subbasin, and the south Perris subbasin (fig.1). The Winchester ground-water subbasin is about $25 \mathrm{mi}$ southeast of Riverside, California, in the upper Santa Ana River drainage basin. The 13$\mathrm{mi}^{2}$ subbasin (California Department of Water Resources, 1979) is bounded by granitic and undifferentiated metamorphic rocks (California Department of Water Resources, 1959). The boundaries of the Winchester subbasin coincide with surface-water-drainage divides, except in areas where valley-floor alluvium is contiguous with neighboring basins. The boundary with the south Perris subbasin to the northwest is arbitrarily selected (California Department of Water Resources, 1979) as the valley constriction west of Double Butte. The boundary with the Menifee subbasin to the southwest is the valley constriction $3.5 \mathrm{mi}$ west of Winchester and about $1 \mathrm{mi}$ northeast of Menifee Lakes (fig.1) (California Department of Water Resources, 1979). Saturated alluvium that fills the constrictions connects the subbasins hydraulically in the subsurface. Alluvium in the Winchester subbasin is estimated to be as much as 500 to $800 \mathrm{ft}$ thick in places (Biehler and Lee, 1994; California Department of Water Resources, 1978). Depth to water generally is about 15 to 75 $\mathrm{ft}$ (California Department of Water Resources, 1978).

Surface water in the Winchester subbasin drains to ephemeral Salt Creek, which is one of few well-defined drainages in the upper Santa Ana River basin. Salt Creek flows westward from the Winchester subbasin, through the Menifee subbasin, and into Railroad Canyon Reservoir.

The $40-\mathrm{mi}^{2}$ Menifee subbasin is bounded by metamorphic rocks on the west and by igneous rocks on the south and east (NBS/Lowry, 1987). The boundary with the Winchester subbasin is to the northeast. The boundary with the south Perris subbasin to the north is arbitrarily set about $1 \mathrm{mi}$ north of Sun City (California Department of Water Resources, 1979). Alluvium in the Menifee subbasin is about $600 \mathrm{ft}$ thick in the Sun City area and more than $800 \mathrm{ft}$ thick in the vicinity of Menifee Lakes (Biehler and Lee, 1995; NBS/Lowry, 1987).
The 50-mi ${ }^{2}$ south Perris subbasin is bounded on the west and east by mostly granitic mountains (Lang, 1979). The south Perris subbasin extends north to the north Perris subbasin, approximately 3 mi north of the study area. The boundaries with the Menifee and Winchester subbasins are to the south and southeast, respectively. Alluvium in the south Perris subbasin is more than $800 \mathrm{ft}$ thick in places, on the basis of drill logs of wells in the subbasin (table 1) and a geophysical study (Biehler and Lee, University of California, Riverside, written commun., 1996). The San Jacinto River, an ephemeral stream, runs southwestward through the subbasin and into the northern end (not shown in fig. 1) of Railroad Canyon Reservoir.

\section{EXISTING WELL-CONSTRUCTION, WATER-QUALITY, AND WATER-LEVEL DATA}

\section{Well-Construction Data}

The location of 115 wells identified in field reconnaissance of the study area in 1995 is shown in figure 1. Available well-construction information for these wells and depth-to-bedrock data, where applicable, are given in table 1 . Because most wells end in alluvium and do not reach bedrock, determination of depth to bedrock solely on the basis of well-log data is difficult. Most wells for observation or for domestic use were constructed with plastic casings, and wells used for irrigation, now or in the past, were constructed with steel casings.

\section{Water-Quality Data}

Historical water-quality data compiled from available USGS, EMWD, and well-owner records are given in table 2. The data include physical properties and concentrations of major ions, nutrients, and trace metals. Many of these wells no longer exist and are not shown in figure 1. Generally, only one set of data per site per year from non-USGS sources is given in table 2.

Water-quality analyses of ground-water samples collected from different zones were done at some sites. These data were obtained by two methods. The first method involved the use of 


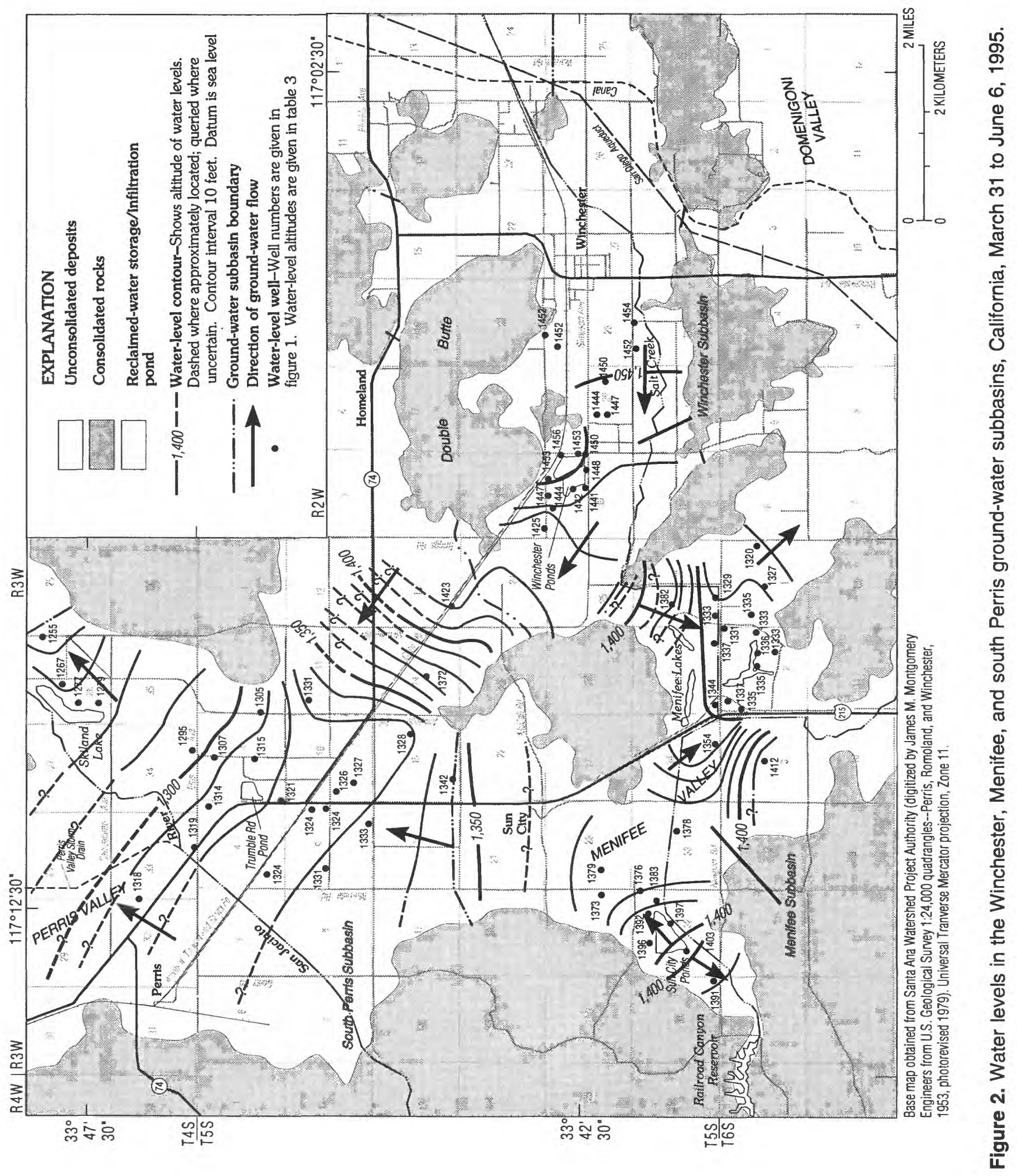




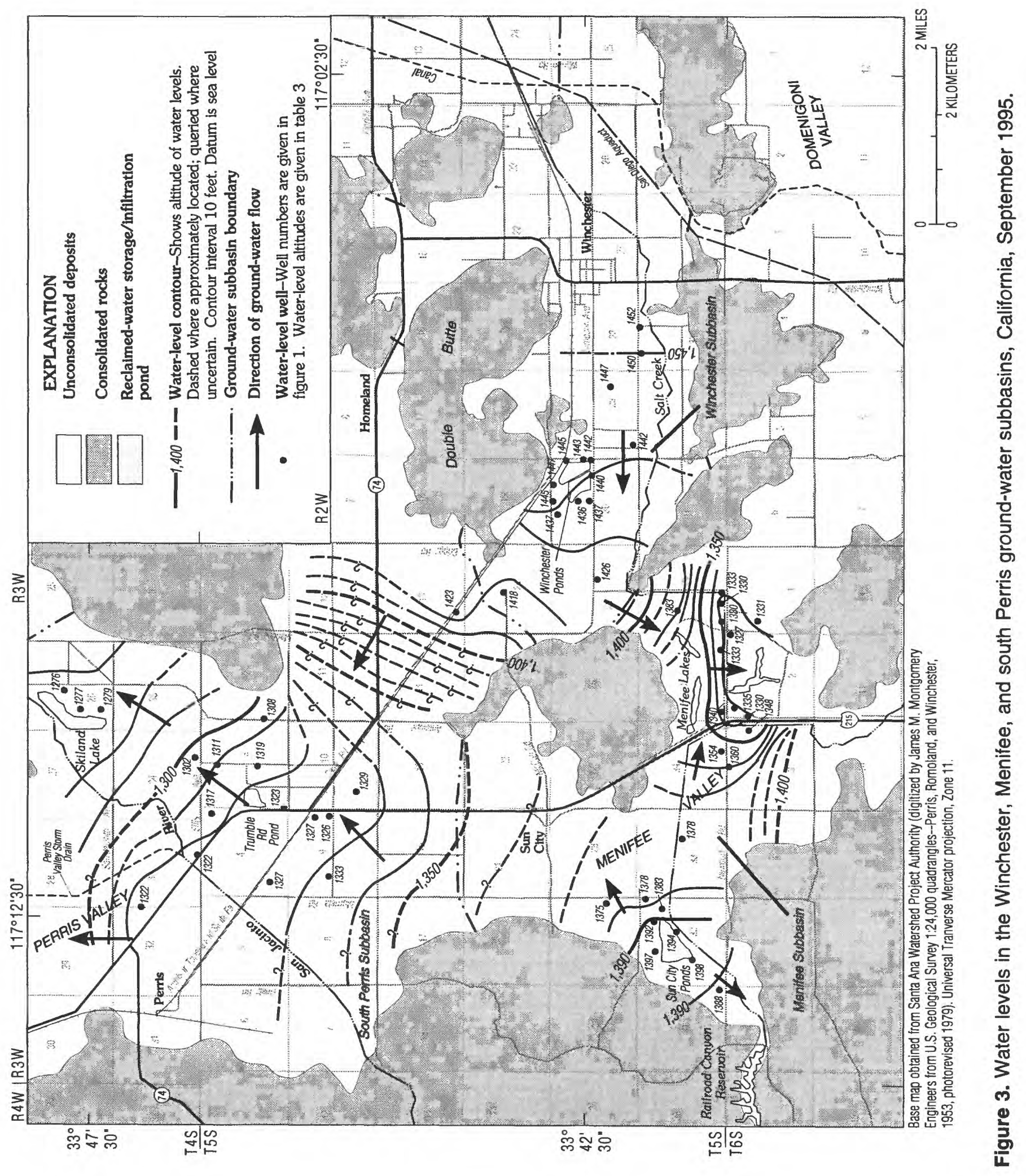


nested piezometers. Three or four 2-inch-diameter well casings with screened intervals at different depths (separated by bentonite grout) were placed in the same borehole. This method was used by the USGS at two sites: wells 5S/3W-28M1, -28M2, $-28 \mathrm{M} 3$, and $-28 \mathrm{M} 4$, and wells 5S/3W-24F2, $-24 \mathrm{~F} 3$, and $-24 \mathrm{~F} 4$. The second method involved the use of inflatable packers placed in multiple-screened casings above and below the desired zone or zones. This method was used by EMWD at five sites: wells $5 \mathrm{~S} / 3 \mathrm{~W}-35 \mathrm{~N} 2$ and $-36 \mathrm{P} 2$, and wells $6 \mathrm{~S} / 3 \mathrm{~W}-$ $2 \mathrm{Al},-2 \mathrm{D} 1$ and $-2 \mathrm{E} 1$. Bentonite grout was used in the annulus to separate the screened intervals in wells $5 \mathrm{~S} / 3 \mathrm{~W}-35 \mathrm{~N} 2$ and $-36 \mathrm{P} 2$. For wells $6 \mathrm{~S} / 3 \mathrm{~W}$ $2 \mathrm{~A} 1,-2 \mathrm{D} 1$, and $-2 \mathrm{E} 1$ the screened intervals were connected by gravel in the annulus.

\section{Water-Level Data}

Water levels in 86 wells were measured during reconnaissance of the study area in 1995 using a steel tape or a calibrated electric sounder. These data, along with historical water-level data collected over several years, are given in table 3 . Generally, for wells that had numerous historical measurements, only one or two measurements per year (usually spring and (or) autumn) are included.

Water levels in wells measured during March 31-June 6, 1995, and again in September 1995, were used to prepare water-level-contour maps (figs. 2 and 3). These contour maps then were used to determine the direction of ground-water flow. Flow is northwestward from the the Winchester subbasin into the south Perris subbasin and southwestward into the Menifee subbasin toward Menifee Lakes. From Menifee Lakes, flow is southeastward. From a ground-water divide near the southwest corner of Sun City storage/infiltration ponds in the Menifee subbasin, ground water flows northward toward the south Perris subbasin and southwestward toward Railroad Canyon Reservoir. In the south Perris subbasin, flow is northward into north Perris subbasin and northeastward into the Lakeview subbasin (north of study area; not shown in figures). Very few differences, other than seasonal changes in altitude of the water-level surface, were found between the two sets (MarchJune 1995 and September 1995) of data.

\section{LITHOLOGIC SECTIONS}

Three generalized lithologic sections (line of sections shown in fig. 4) were constructed to gain a better understanding of the stratigraphy and thickness of alluvial deposits in the Winchester, Menifee, and south Perris subbasins. Alluvial sediment in driller's log descriptions of cuttings was grouped into four categories that are similar to the categories used by Rees and others (1995): (1) fine-grained deposits-including sandy soil, conglomerate, clay with gravel, cemented sand or gravel, and topsoil; (2) sand deposits-including silty sand, silty sand and gravel, and sand with gravel; (3) gravel deposits-including rock and sand, coarse sand and gravel, cobbles, and boulders; (4) lenses of clay, sand, and gravel-including interbedded layers of clay, sand, and (or) gravel. Underlying weathered and unweathered bedrock were grouped into two additional categories, respectively: (5) decomposed granite and (6) granitic basement rock.

The lithology of selected wells along three sections in the study area is shown in figures 5,6 , and 7. Lithologic section $A-A^{\prime}$ (fig. 5) extends from the southwest corner of the Menifee subbasin northeastward through the south Perris subbasin; section $B-B^{\prime}$ (fig. 6) extends from the northwest part of the south Perris subbasin southeastward through the Winchester subbasin; and section $C-C^{\prime}$ (fig. 7) extends west to east through the Menifee subbasin.

\section{POND-INFILTRATION ESTIMATES}

A water-budget method was used to estimate the quantity of water infiltrating from selected storage/infiltration ponds. Measurement of unsaturated-zone moisture content, change in unsaturated- and saturated-zone storage, and aquifer specific yield beneath the ponds would have provided information on the rate that water moves through the unsaturated zone (if present), but such work was beyond the scope, instrumentation, and time limitations of the study.

Water-level data were collected from one pond at each of the three storage-pond sites, primarily during July-September 1995. The data- 


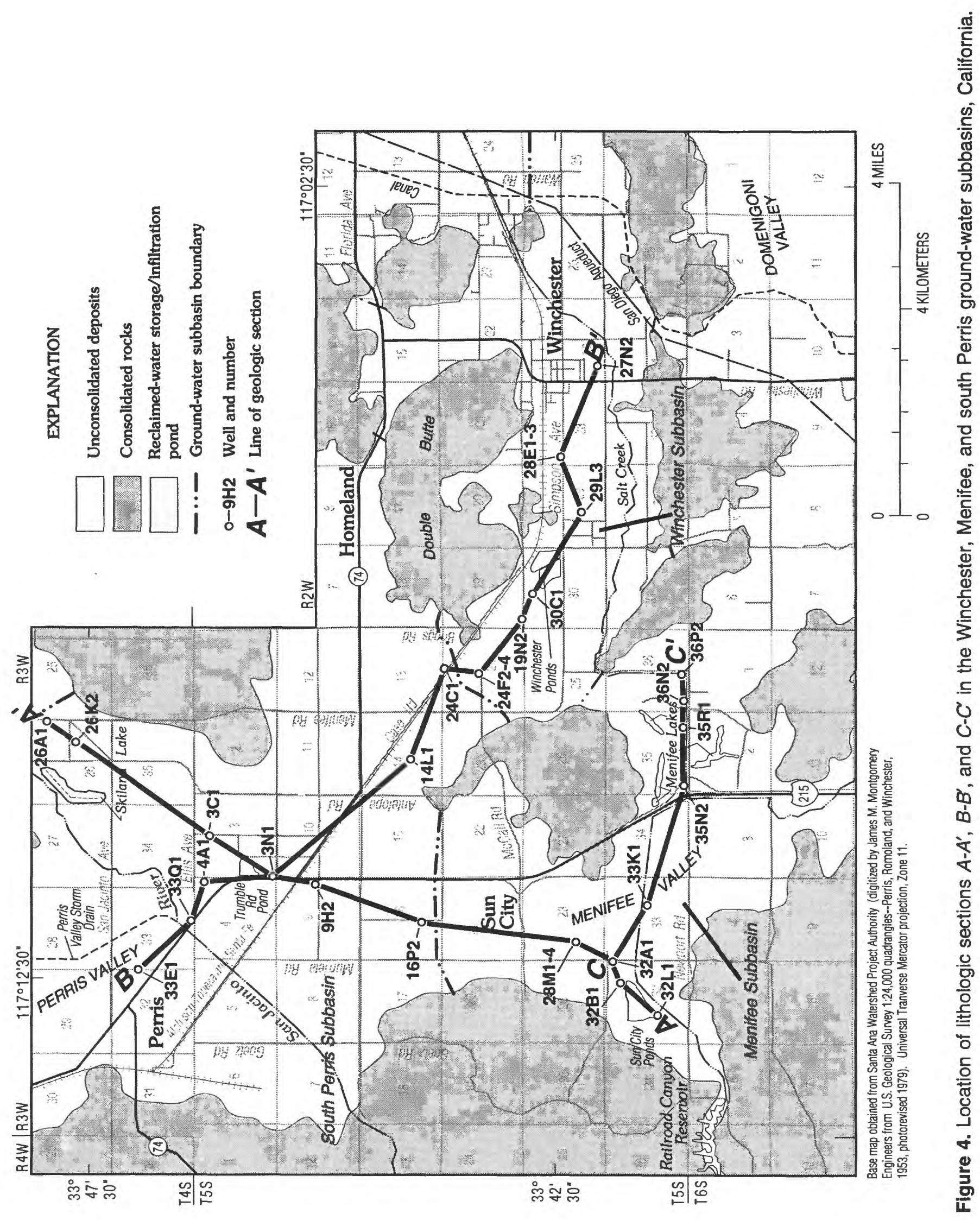




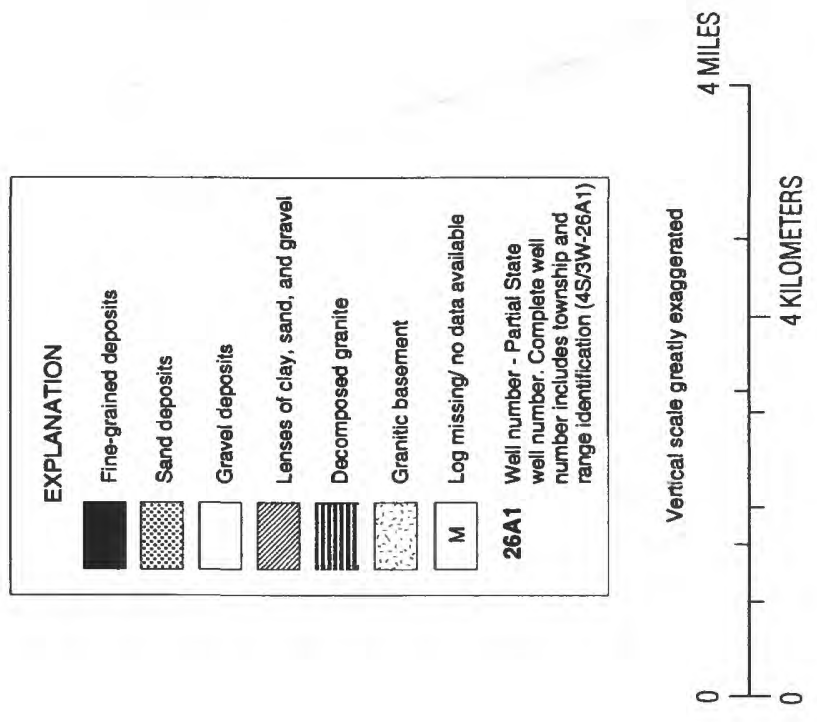

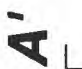

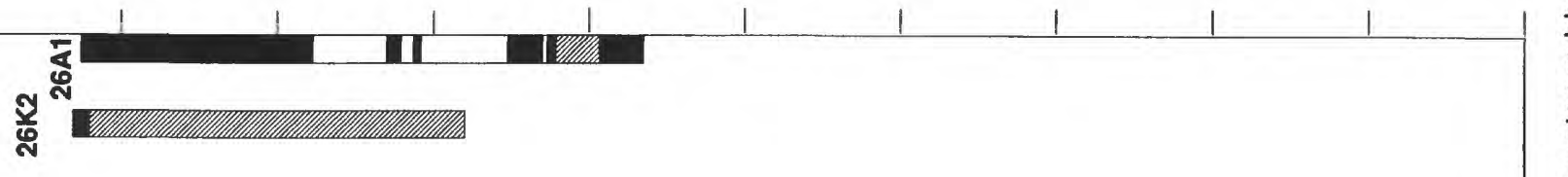

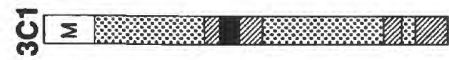

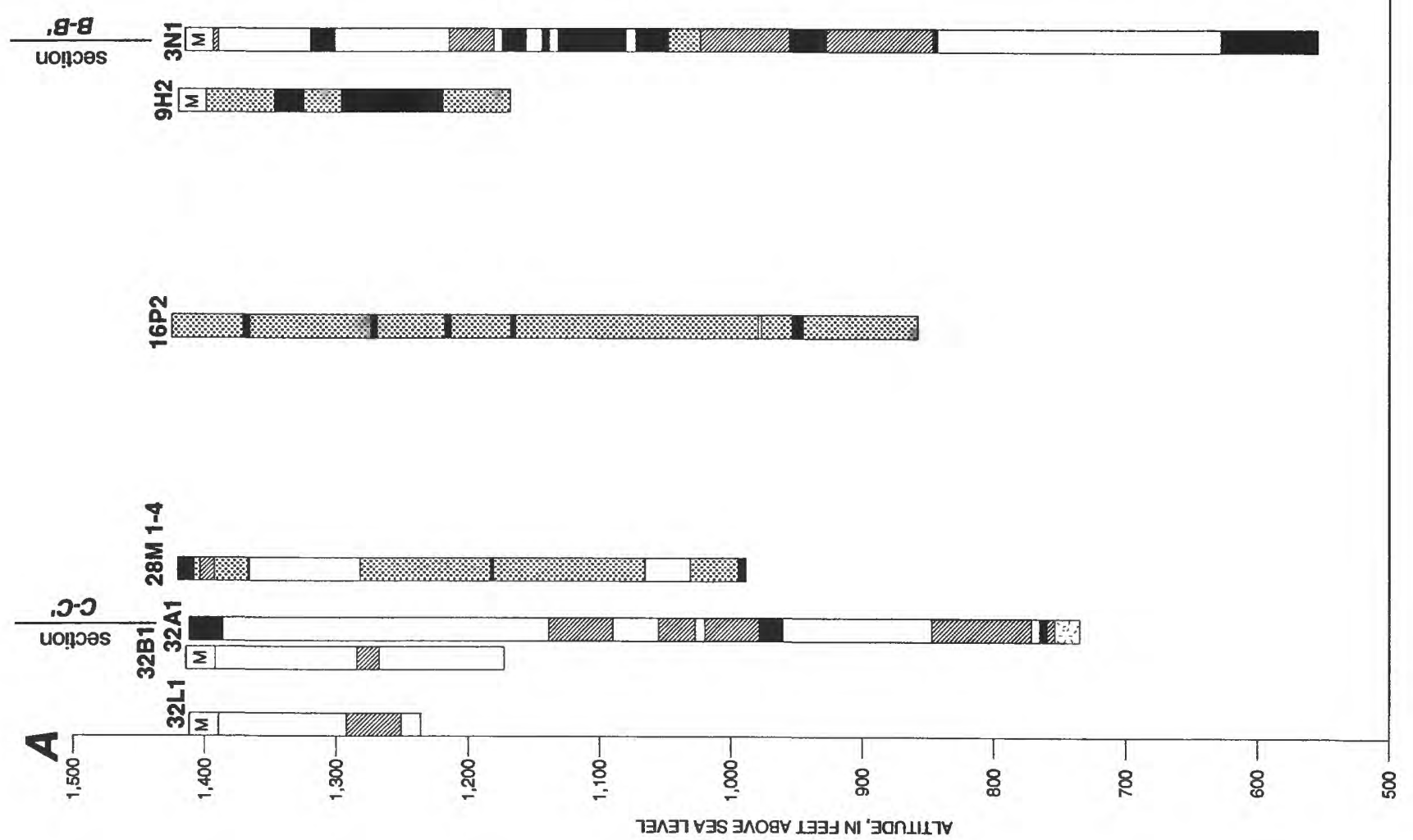

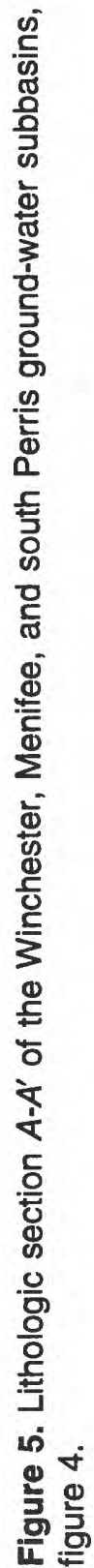



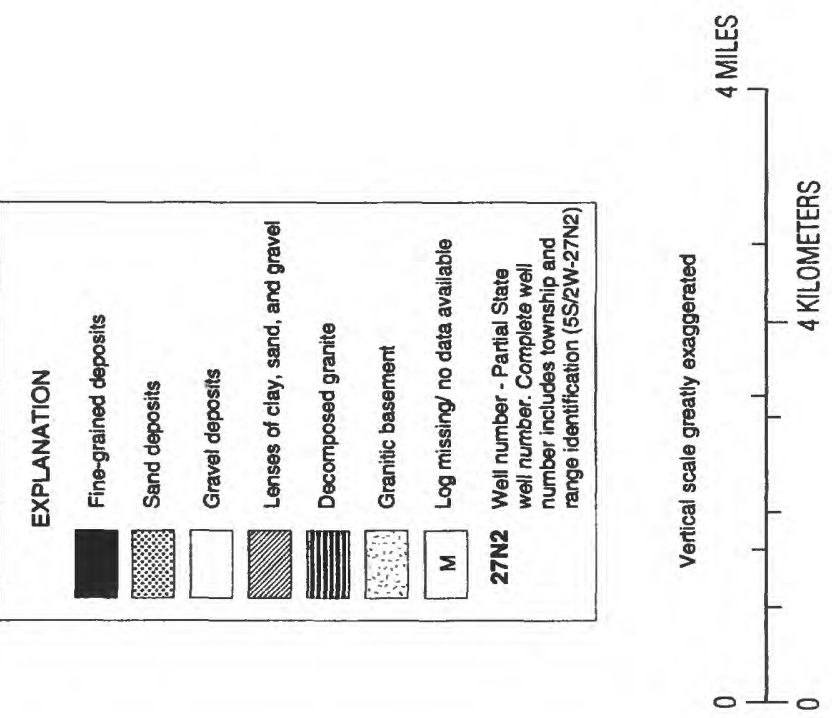

in
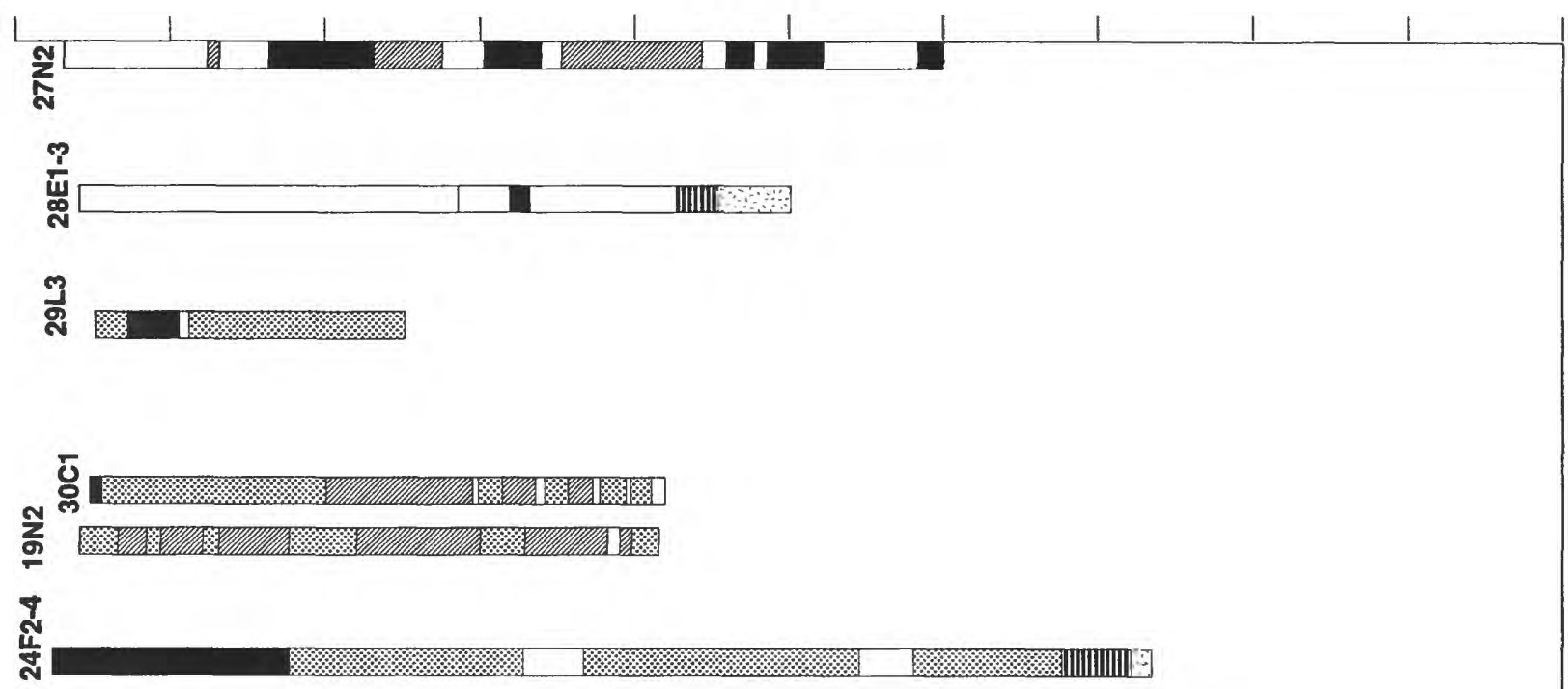

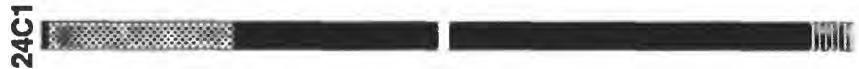

פ
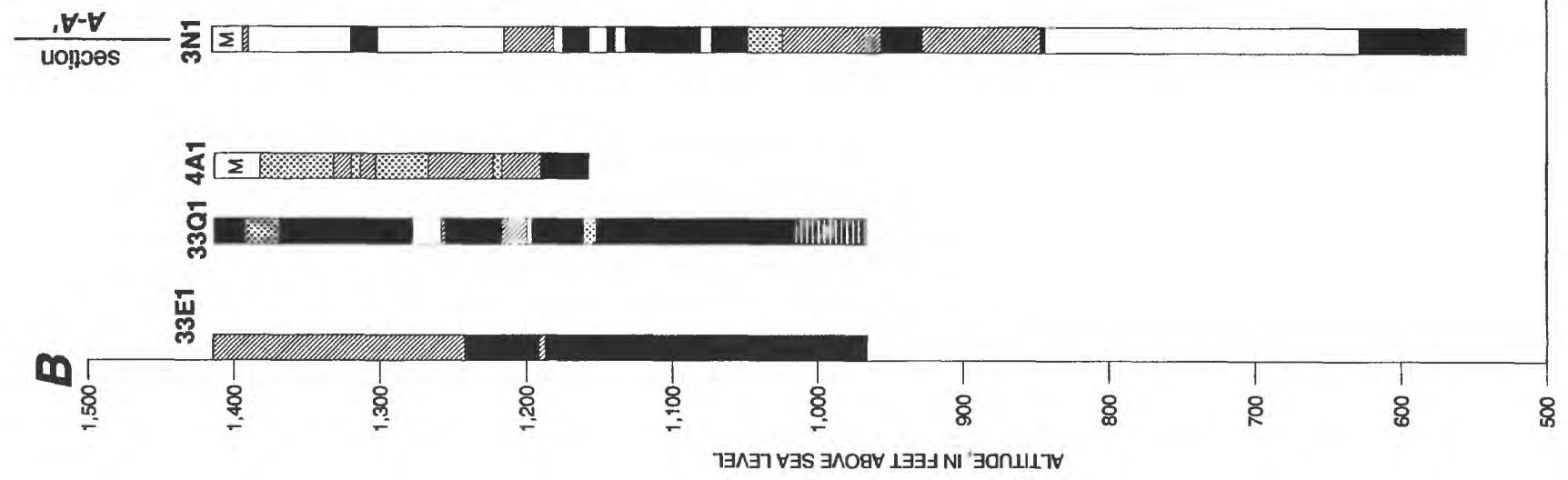

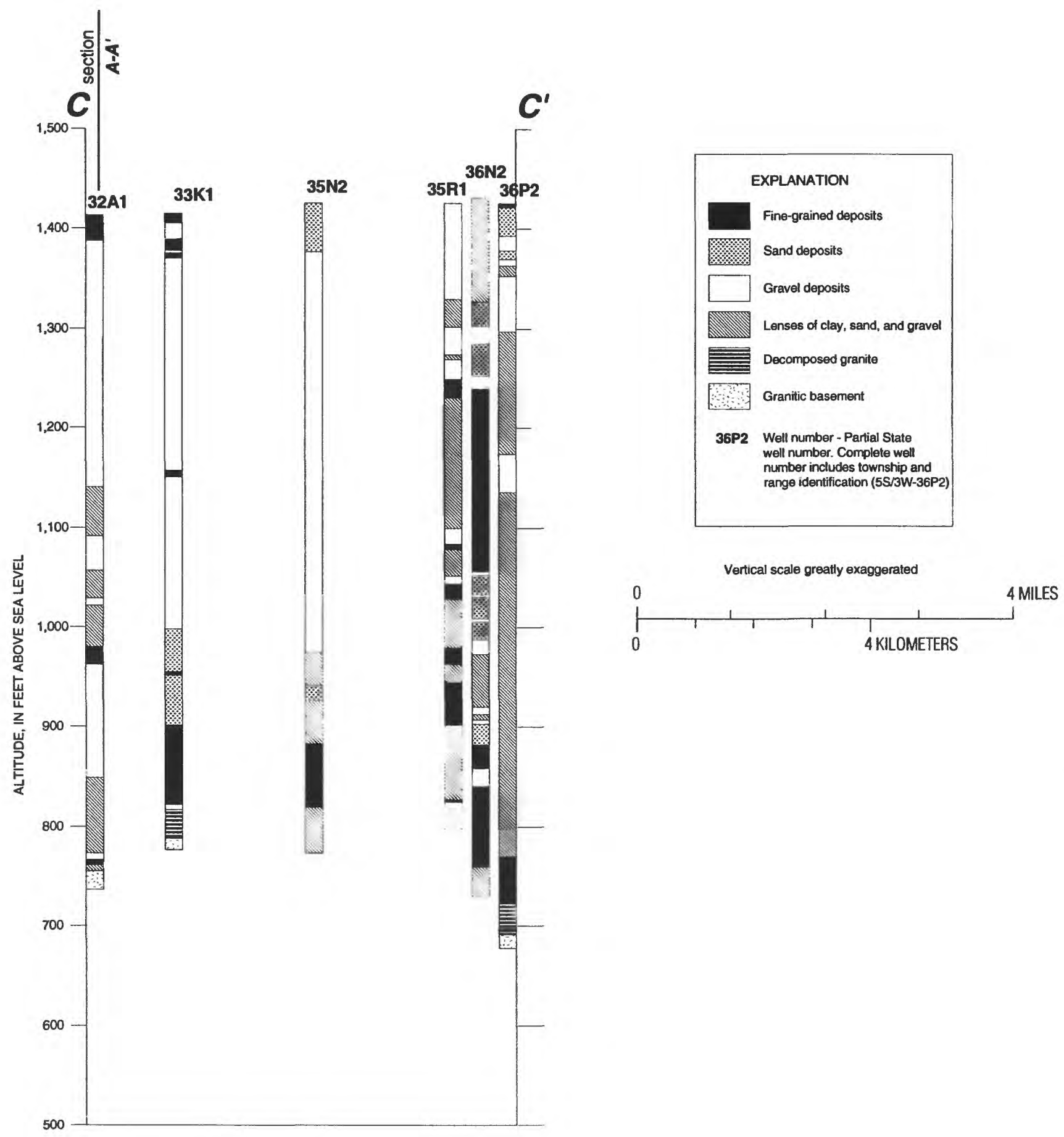

Figure 7. Lithologic section $C-C$ in the Winchester, Menifee, and south Perris ground-water subbasins, California. Line of section is shown in figure 4. 
collection time period was limited by the brief timespan of the study. These data were collected using Rittmeyer submersible pressure transducers installed in 2-inch-diameter stilling wells and connected to Stevens AXSYS SDI-12 data loggers. The transducer data were calibrated against periodic staff-gage measurements of pond levels. Pond infiltration for a chosen time period was estimated using a water-balance equation:

Volume infiltrated $=[$ (starting volume in pond $)$

- (ending volume in pond) $]+[$ (volume pumped

into pond) - (volume pumped out of pond)]

- (evaporation) + (rainfall) .

Starting and ending volumes were determined using the measured pond water levels in conjunction with pond-level/pond-volume conversion tables or graphs provided by EMWD. Volumes pumped into and out of ponds also were measured by EMWD. Evaporation was measured near Lake Skinner at Tucalota Creek, 8 mi south of Winchester, by Metropolitan Water District of Southern California (MWD) using a U.S. Weather Bureau Class A pan. A pan coefficient of 0.7 was applied to convert the measured pan value to an estimated value for a lake or reservoir, as is common practice (Veihmeyer, 1964, p. 11-7).

The methods used to calculate the components of equation 1 varied slightly for each of the three sites owing to differences in pond operating procedures and available data. The three sites are discussed individually below. In addition, the pond sites differ somewhat in construction. The Sun City and Trumble Road ponds have unlined bottoms and sides, whereas the Winchester ponds have unlined bottoms and compacted-clay or soilcement sides. Sun City pond no. 9 and the Winchester ponds are about $20 \mathrm{ft}$ deep, and the Trumble Road pond is about $60 \mathrm{ft}$ deep.

\section{Sun City Ponds}

Pond no. 9 at the Sun City RWRF (fig. 8A) was instrumented with a Rittmeyer Series MPSDI submersible pressure transducer placed in a 2-inchdiameter stilling well and connected to a Stevens AXSYS SDI-12 data logger in June 1995. This pond was chosen because during normal operation the pond is not pumped, and the only inflow it receives is occasional overflow from pond no. 8 during the winter months; thus, the number of variables needed to calculate outflow in the form of infiltration to ground water is reduced. However, at about the same time the instrumentation was installed, the pond was pumped (June 1-July 24, 1995) to lower the water level of the pond and the immediately adjacent water table in preparation for construction of a buried pipeline next to the pond.

Pond water levels, measured in pond no. 9 beginning 10 days after pumping ceased, rose 0.68 ft during August 3-September 15, 1995 (fig. 9 and table 4). During this period, no water was pumped into or out of the pond; therefore, the rise in water level represents the combined effects of upward percolation of ground water, minus evaporation, plus rainfall. The upward percolation of water probably is the result of the lowering of the water level in pond no. 9, during pumping, to an altitude below the local water-table altitude (figs. $10 \mathrm{~A}$ and $11 A$ ), followed by a gradual rise as the pond level equilibrated with the water-table altitude.

The local water table (and pond no. 9 water level) probably was influenced by infiltration of water from pond no. 8 and pond no. 7 (fig. $8 A$ ), which were kept relatively full. Generally, treated water is pumped from the RWRF to ponds nos. 1-4, and then pumped to pond no. 7 approximately twice per week. During the winter, some of this water cascades, at times, into pond no. 8 and pond no. 9 . Shown in table 5 are water-level altitudes in observation wells near the Sun City ponds for April-September 1995. It should be noted that although these wells are perforated at a depth of 200 to $220 \mathrm{ft}$, which is about $180 \mathrm{ft}$ below the water table, the water levels nevertheless might be representative of the water table rather than a deeper confined aquifer (except for well $5 \mathrm{~S} / 3 \mathrm{~W}$ $32 \mathrm{~A} 1$, which is perforated at a depth of 560 to 580 $\mathrm{ft}$ ). The decline in water level in well $5 \mathrm{~S} / 3 \mathrm{~W}-32 \mathrm{~L} 1$ (table 5) probably was influenced by the pumped decline in water level in pond no. 9; conversely, the rise in water level in well $5 \mathrm{~S} / 3 \mathrm{~W}-32 \mathrm{C} 1$ probably is related to the twice-weekly addition of treated water to pond no. 7. (These additions kept the pond relatively full.) The second largest decline in water level, in well 5S/3W-32Gl (table 5; fig. 8A), probably reflects the dry status of pond no. 1 and pond no. 5 during the measurement period. The rise in water level of $1.61 \mathrm{ft}$ in well $5 \mathrm{~S} / 3 \mathrm{~W}-32 \mathrm{~A} 1$ may be indicative of regional change in a deeper aquifer. 

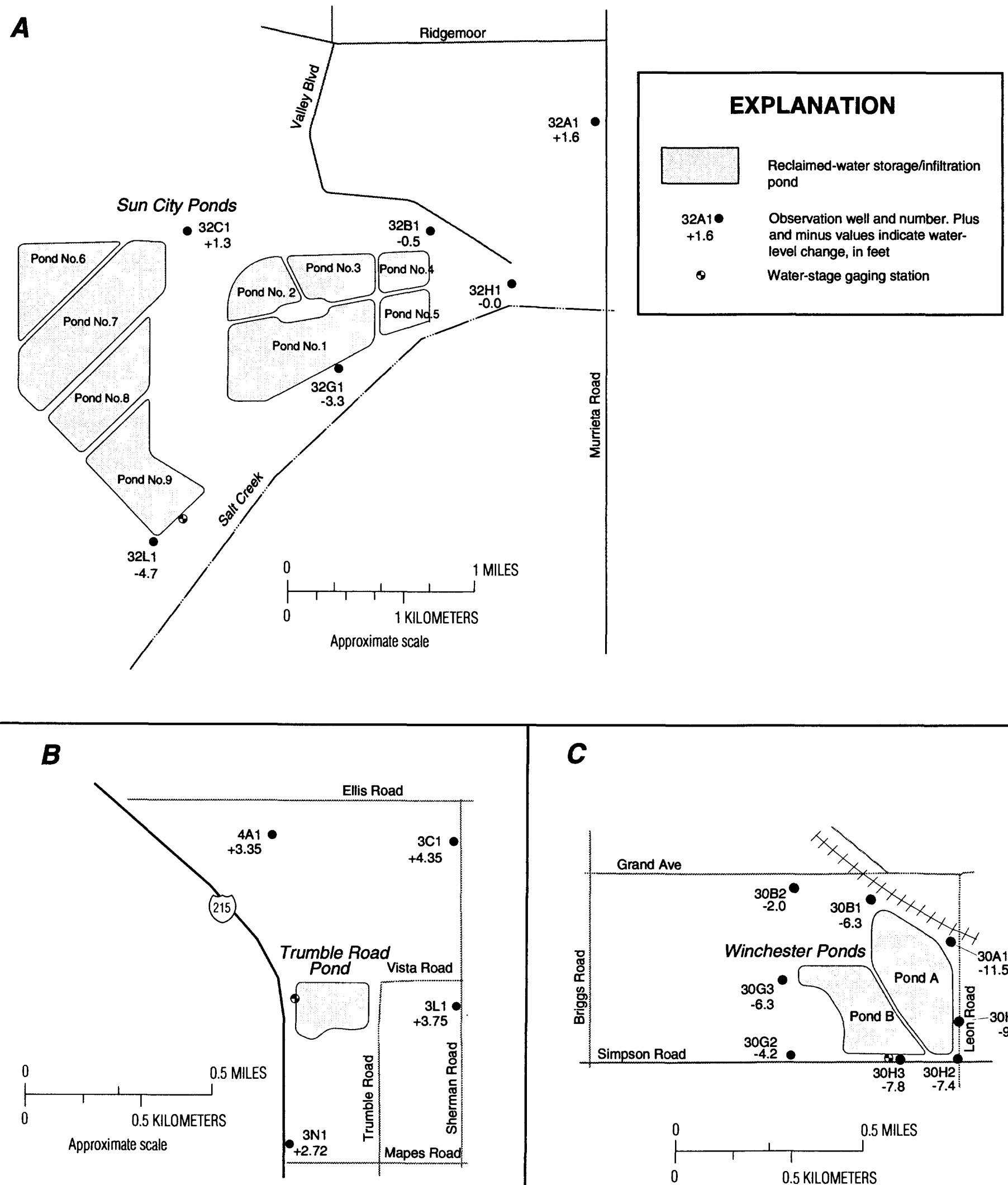

\section{C}
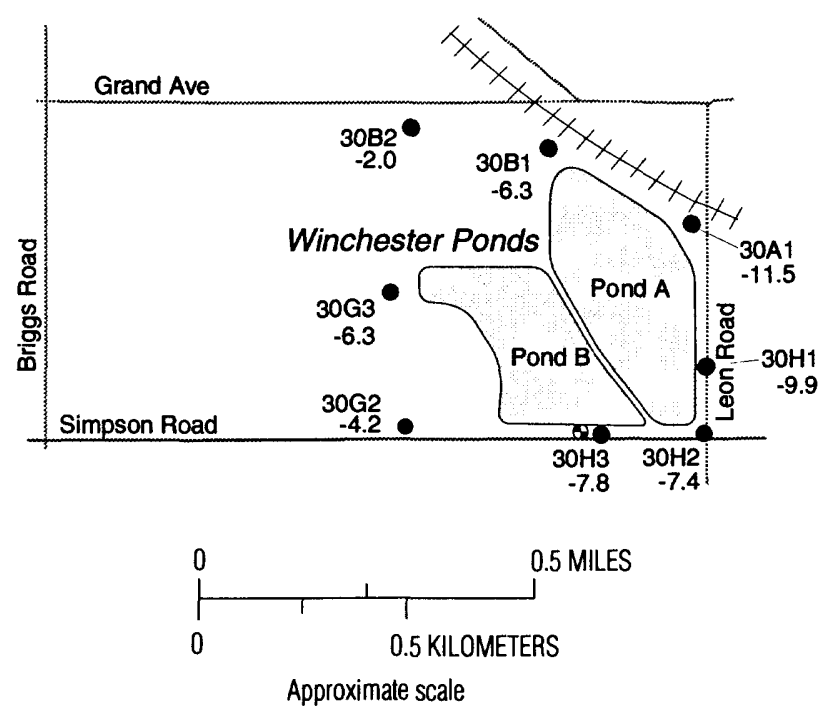

Figure 8. Pond sites, ground-water-level changes, and location of observation wells and water-stage gaging stations. A. Sun City site; ground-water-level change from 4/25/95 to 9/13/95; B. Trumble Road site; groundwater-level change from $3 / 31 / 95$ or $4 / 14 / 95$ to $9 / 11 / 95$ or $9 / 13 / 95$; C. Winchester site; ground-water-level change from $5 / 11 / 95$ or $5 / 23 / 95$ to $9 / 11 / 95$ or $9 / 13 / 95$. (See figure 1 for site locations.) 


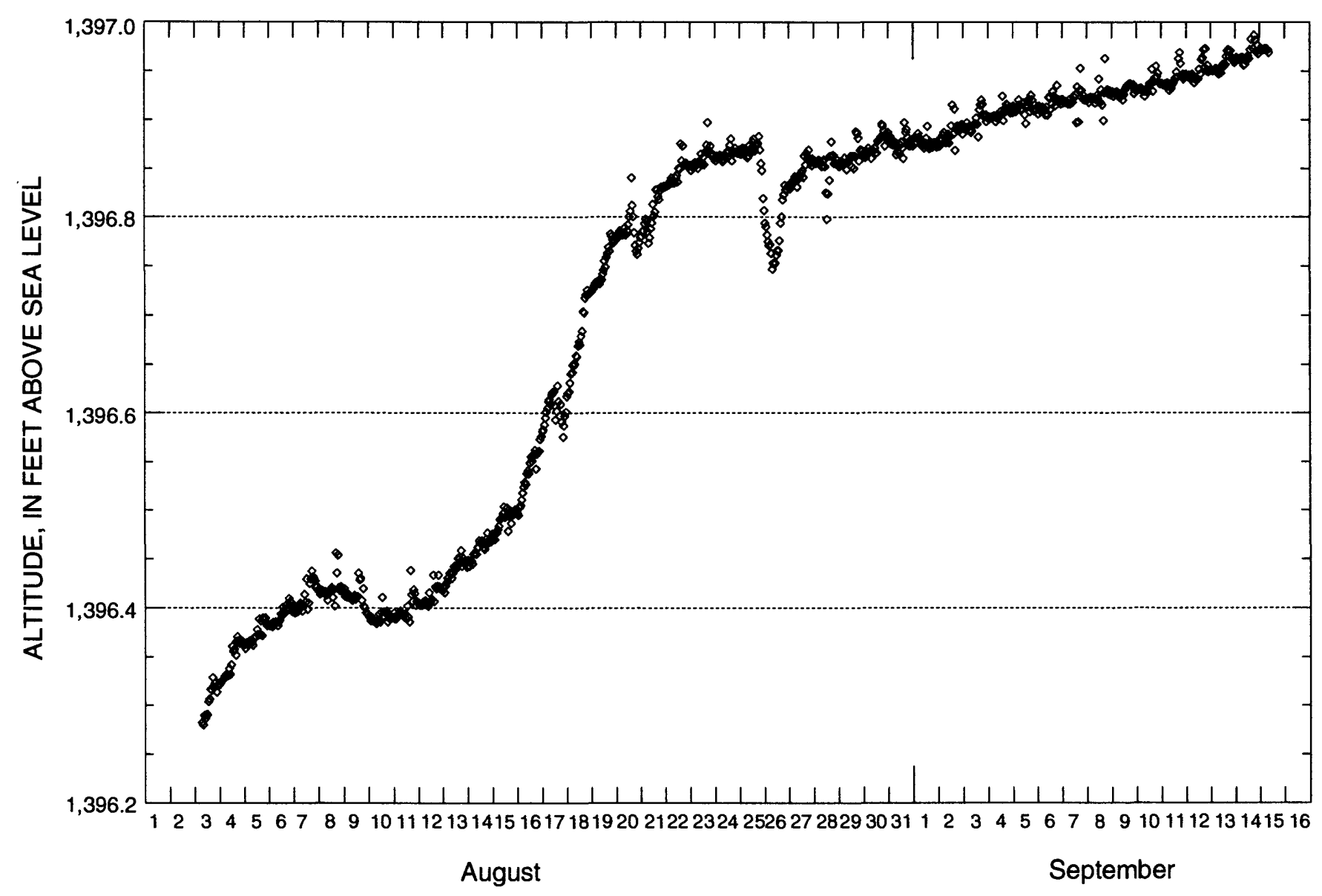

1995

Figure 9. Water-level altitudes in Sun City pond no. 9, August 3 to September 15, 1995.

The purpose in measuring water levels in pond no. 9 was to use the change in volume of water in a water-balance equation, and to solve for the volume of water that infiltrated from the pond. For the period August 3 to September 15, 1995 (fig. 9 ), no water was pumped into or out of the pond and there was no rainfall; therefore, equation 1 becomes:

Volume infiltrated $=[$ (starting volume in pond $)$

- (ending volume in pond)] - (evaporation).

Water levels in the pond were converted to volumes using a pond-stage/pond-volume conversion table (table 6) provided by EMWD (Alfred Javier, EMWD, written commun., 1996):

Water level on August 3, 1995 (8 a.m.) = $1,396.28 \mathrm{ft}$;

water level on September 15, 1995 (8 a.m.) = $1,396.97 \mathrm{ft}$.
From table 6: For pond stage of $1,396.5 \mathrm{ft}$, pond volume $=10,500,000 \mathrm{gal}=1,403,700 \mathrm{ft}^{3}$; and for pond stage of $1,396.0 \mathrm{ft}$, pond volume $=9,600,000 \mathrm{gal}=1,283,400 \mathrm{ft}^{3}$.

Rate of change $=120,300 \mathrm{ft}^{3}$ per $0.5 \mathrm{ft}$ of water-level change $=240,600 \mathrm{ft}^{3} / \mathrm{ft}$ of water-level change.

Therefore, pond volume at a pond stage of $1,396.28 \mathrm{ft}=\left(1,283,000 \mathrm{ft}^{3}\right)+(0.28 \mathrm{ft} \times$ $\left.240,600 \mathrm{ft}^{3} / \mathrm{ft}\right)=1,350,000 \mathrm{ft}^{3}$.

From table 6: For pond stage of $1,397.0 \mathrm{ft}$, pond volume $=11,300,000 \mathrm{gal}=1,511,000 \mathrm{ft}^{3}$; and for pond stage of $1,396.5 \mathrm{ft}$, pond volume $=10,500,000 \mathrm{gal}=1,404,000 \mathrm{ft}^{3}$.

Rate of change $=107,000 \mathrm{ft}^{3}$ per $0.5 \mathrm{ft}$ of water-level change $=214,000 \mathrm{ft}^{3} / \mathrm{ft}$ of water-level change. 
Therefore, pond volume at a pond stage of $1,396.97 \mathrm{ft}=\left(1,511,000 \mathrm{ft}^{3}\right)-(0.03 \mathrm{ft} \times$ $\left.214,000 \mathrm{ft}^{3} / \mathrm{ft}\right)=1,505,000 \mathrm{ft}^{3}$.

Evaporation for August 3 to September 15, 1995, was estimated by multiplying the summed daily pan- evaporation values, measured by Metropolitan Water District (MWD) at Tucalota Creek near Lake Skinner (about 13 mi southeast of Sun City), by the appropriate rate of volume change at the prevailing water-level altitude in pond no. 9 and by a pan coefficient of 0.7 :

Feet of evaporation $=(14.23$ in $) \times(0.7)=$ $9.96 \mathrm{in}=0.83 \mathrm{ft}$;

Rate of pond-volume change at 1,396 to $1,397 \mathrm{ft}$ altitude $=\left(240,600 \mathrm{ft}^{3} / \mathrm{ft}+\right.$ $\left.214,000 \mathrm{ft}^{3} / \mathrm{ft}\right) / 2$;

Volume evaporated $=(0.83 \mathrm{ft}) \times$

$\left[\left(240,600 \mathrm{ft}^{3} / \mathrm{ft}+214,000 \mathrm{ft}^{3} / \mathrm{ft}\right) / 2\right]=$ $188,700 \mathrm{ft}^{3}$.

Substitution into equation 2 gives:

Volume infiltrated $=\left[\left(1,350,000 \mathrm{ft}^{3}\right)\right.$ -

$\left.\left(1,505,000 \mathrm{ft}^{3}\right)\right]-\left(0.83 \mathrm{ft} \times 227,300 \mathrm{ft}^{3} / \mathrm{ft}\right)$

$=\left[-155,000 \mathrm{ft}^{3}\right]-\left(188,700 \mathrm{ft}^{3}\right)=$

$-343,700 \mathrm{ft}^{3}=-7.89$ acre-ft.

The average rate of infiltration for August 3September 15 is -0.18 acre-ft/d (upward infiltration.) The average rate for August 28 to September 15 , a period of constant rate of water-level change (fig. 9), is -0.15 acre-ft/d. The above infiltration rates were estimated using data collected during conditons of low pond stage and probably are not representative of conditions at higher stage. Ranges in possible error in the estimates are discussed in the section "Uncertainties in Infiltration Estimates."

The calculation of infiltration rate is dependent, in part, on the accuracy of the evaporation estimates. As an alternative to using pan-evaporation rates, water-level altitudes for three 5-day periods for pond no. 9 were examined to see if the daytime/nighttime difference in rate of waterlevel change could be used to estimate evaporation. The data for three 5-day periods (fig. 12; table 7) did not show the expected daily increased rate of water-level rise at night when evaporation might be considered to be negligible (or at least greatly reduced). Instead, the peak daily water-level altitudes usually occurred between 1300 and 2000, a period that generally includes the hottest and windiest times of day. Therefore, this method for estimating evaporation was not used. Possible theoretical explanations (and arguments against them) for the observed timing of daily peak water levels may be (1) water added to other ponds at the site once per day (water was added only three times [to pond no. 7] during the 15 days); (2) rainfall at about the same time every day (there was no rainfall on any of the days); (3) wind-driven water "piling up" toward one side of the pond; (4) transducer error caused by heating of the cable by air-temperature increases (peaks do not correspond as closely as would be expected to daylight hours); (5) tidal effects (the response does not appear to be diurnal); or, (6) barometric-pressure effects. In essence, an adequate explanation for the observed pattern of daily water-level rises has not been found, but some effect related to temperature or wind appears to be most likely.

\section{Trumble Road Pond}

The single pond at the Trumble Road site (fig. 8B) was instrumented with a Rittmeyer Series MPSDI submersible pressure transducer placed in a 2-inch-diameter stilling well and connected to a Stevens AXSYS SDI-12 data logger, and waterlevel data were collected during July 22-September 27,1995 . This period was chosen, in part, because no water was pumped into the pond after the beginning of July. The data (fig. 13; table 8) show a decline in water level of about $30 \mathrm{ft}$ during the period. The drawdown was caused in large part by continuous pumping of the pond at a rate of approximately 960 to $1,030 \mathrm{gal} / \mathrm{min}$. Direction of ground-water movement in the vicinity of the pond did not vary seasonally as much as at the Sun City and Winchester sites (figs. 10 and 11).

The exception to continuous pumping was one 24-hour period-from 0914 on September 11, 1995, to 0930 on September 12, 1995-when the pond operators were able to shut down the pump. Water-level data for this period of reduced number of inflow and outflow variables can be seen as a relatively flat section in the graph in figure 13 and are shown in detail in figure 14. The rate of infiltration, estimated on the basis of the slope of line segment CD (12 hours) in figure 14 , is 0.72 acre-ft/d (equivalent to $260 \mathrm{acre}-\mathrm{ft} / \mathrm{yr}$ and 235,000 $\mathrm{gal} / \mathrm{d}$ ), and was estimated as follows: 
A
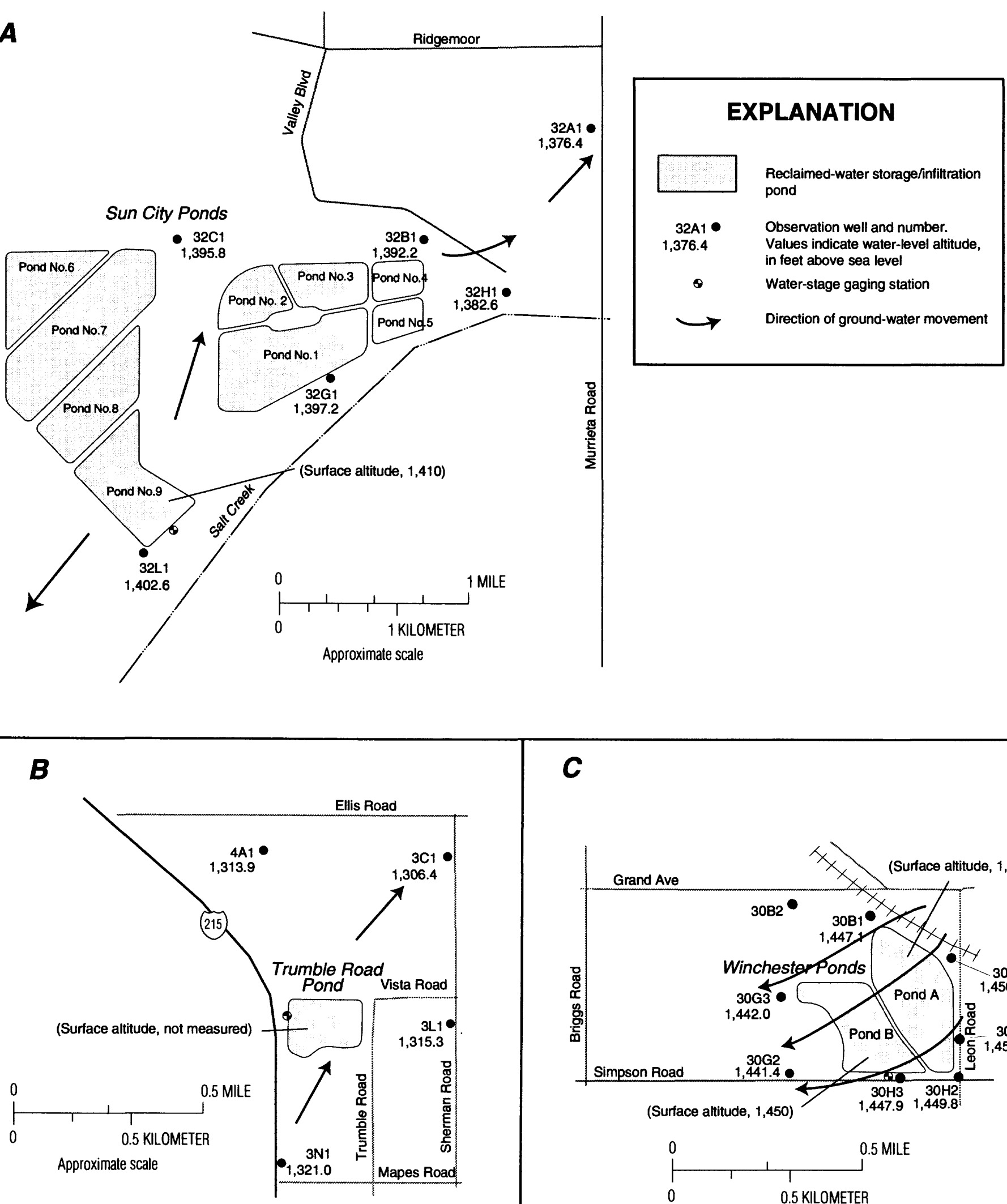

C
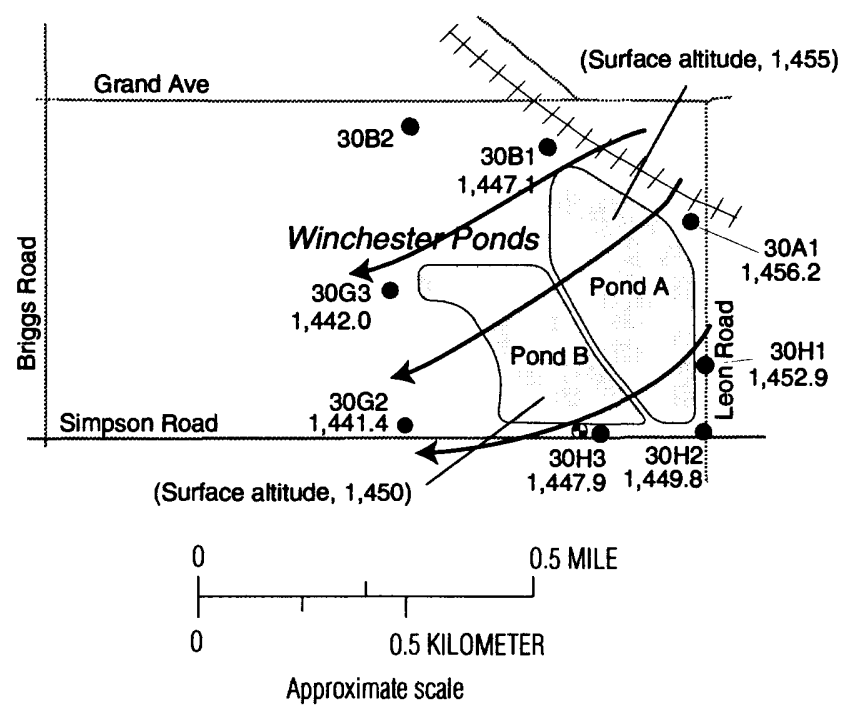

Figure 10. Water-level altitudes, spring 1995. A. Sun City site, 4/25/95; B. Trumble Road site, 3/31/95; C. Winchester site, 5/11/95 to 5/23/95. (See figure 1 for site locations.) 
$\boldsymbol{A}$
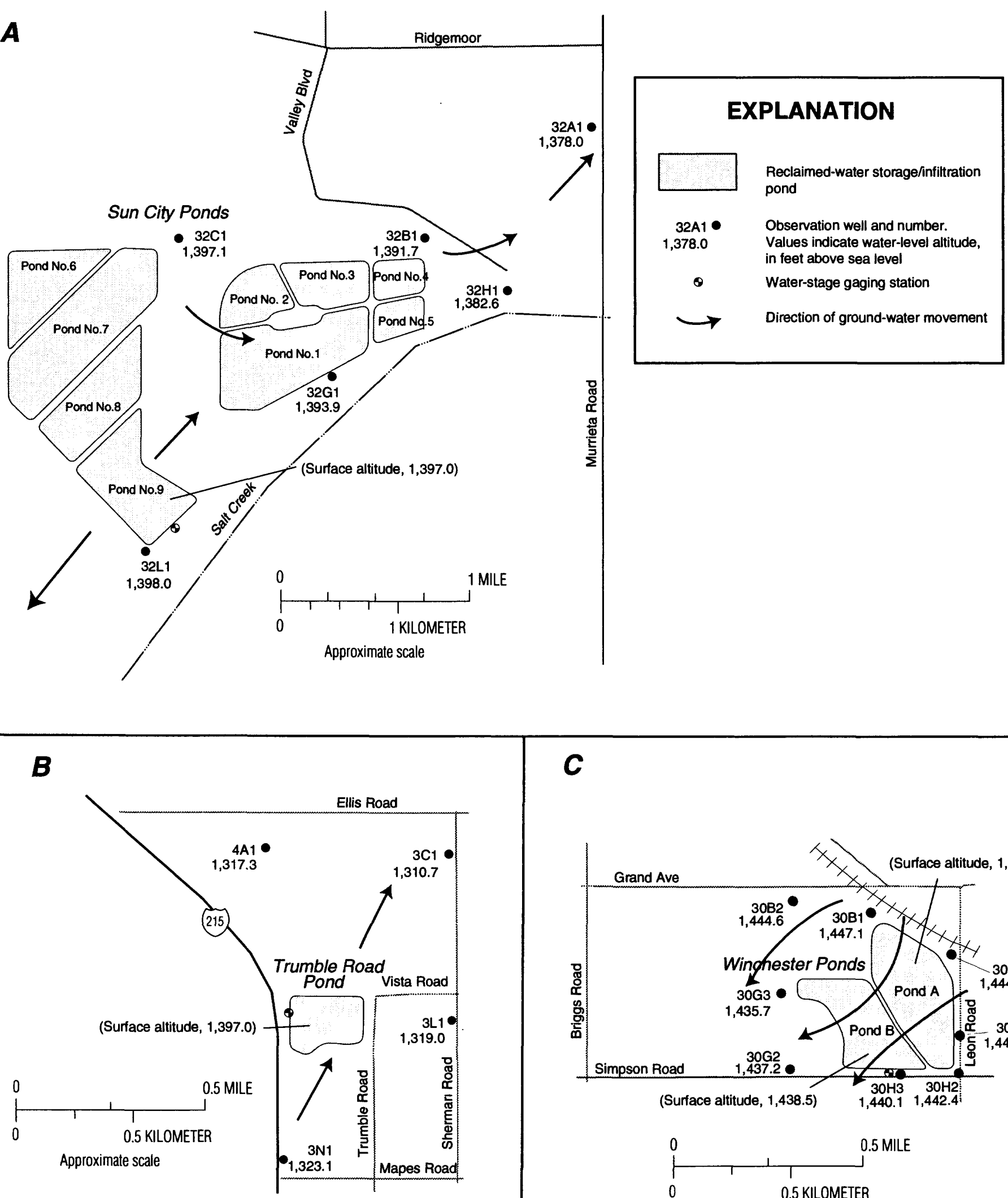

\section{C}

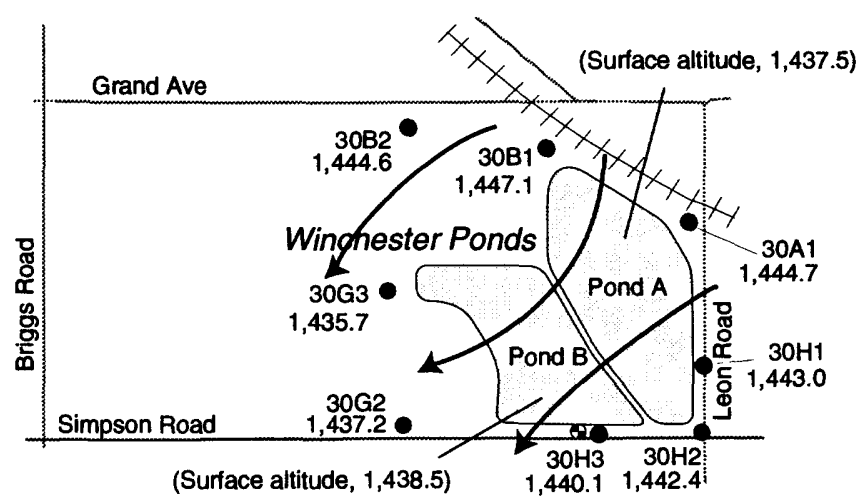

(Surface altitude, 1,438.5)

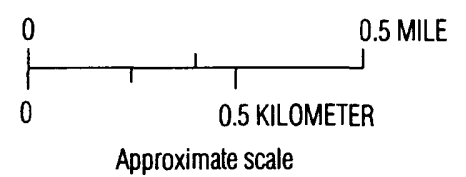

Approximate scale

Figure 11. Water-level altitudes, autumn 1995. A. Sun City site, 9/13/95; B. Trumble Road site, 9/11/95; C. Winchester site, $9 / 11 / 95$ to $9 / 13 / 95$. (See figure 1 for site locations.) 


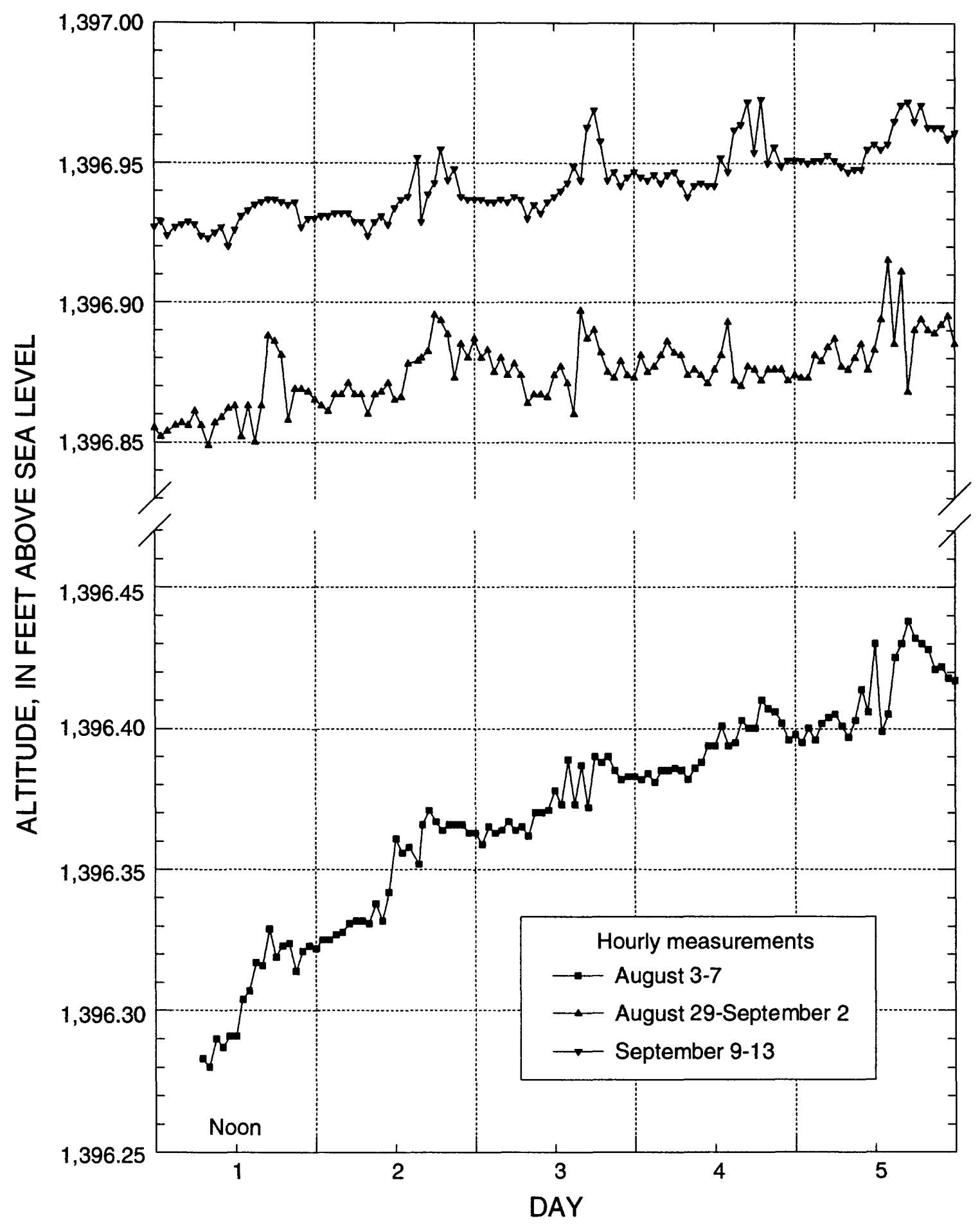

Figure 12. Water-level altitudes in Sun City pond no. 9 for three 5-day periods. 


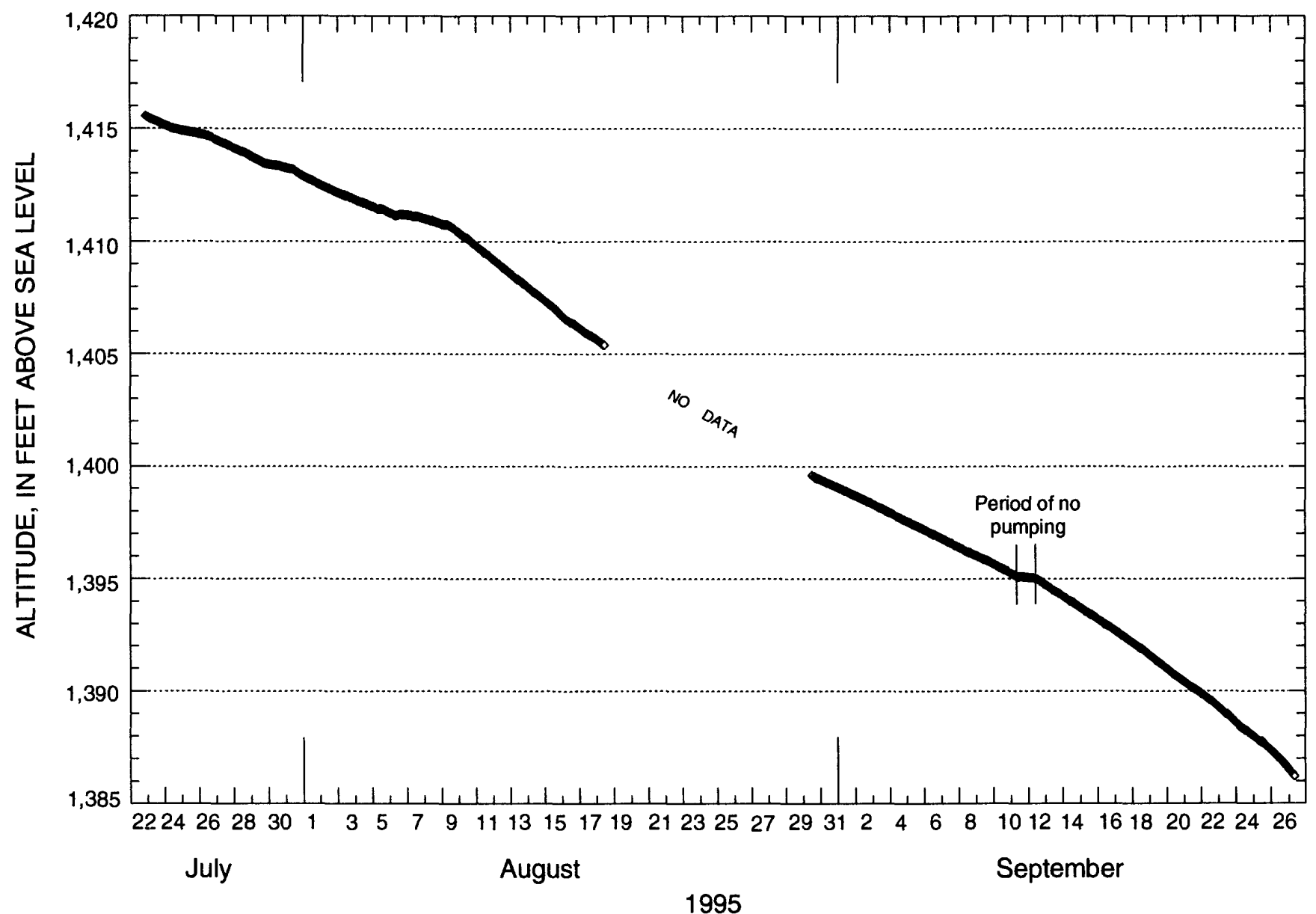

Figure 13. Water-level altitudes in Trumble Road pond, July 22 to September 27, 1995.

12-hour change in pond level $=$

$(1,395.08 \mathrm{ft}-1,395.04 \mathrm{ft})=0.04 \mathrm{ft}$.

Rate of change in volume to change in water level: Volume at water level $1,395.6 \mathrm{ft}=1.100 \times$ $10^{8} \mathrm{gal}$, and volume at water level $1,394.0 \mathrm{ft}=$ $1.053 \times 10^{8}$ gal (Alfred Javier, EMWD, written commun., 1996);

Rate $=\left(4.75 \times 10^{6} \mathrm{gal} / 1.6 \mathrm{ft}\right) \times(0.04 \mathrm{ft} / 0.5 \mathrm{~d})$ $\mathrm{x}(1 \mathrm{acre}-\mathrm{ft} / 325,000 \mathrm{gal})=0.72 \mathrm{acre}-\mathrm{ft} / \mathrm{d}$.

Segment $C D$, which represents change in water level between 1800 and 0600 , is best suited for estimating infiltration rate because the effect of evaporation is assumed to be minimized. Segments $\mathrm{AB}$ and $\mathrm{FG}$ (fig. 14) represent the rate of waterlevel change while the pond was being pumped, and segment DE may include the effect of increased evaporation after sunrise.
The above rates calculated using segment CD are overestimated if evaporation was responsible for a significant part of the water-level decline observed between 1800 and 0600 ; calculations were made using the assumption that evaporation was negligible. The average pan-evaporation rate, measured $11 \mathrm{mi}$ southeast of the pond, for September 11, 1995 ( 24 hours), was $0.02 \mathrm{ft} / \mathrm{d}$ (Richard Morton, EMWD, written commun., 1996) after application of a pan coefficient of 0.7 . The pan coefficient is used to convert observed pan-evaporation values to estimated values for reservoirs or lakes, and it takes into account the thermal effects of the large volume of pond water in comparison with the small volume of the pan. Reported pan coefficients vary from 0.6 to 0.8 (Veihmeyer, 1964, p. 11-7). For the evening and nighttime period represented by segment $C D$, the adjusted 24-hour average pan-evaporation rate is greater than actual evaporation from the pond surface because the pond measurements were made 


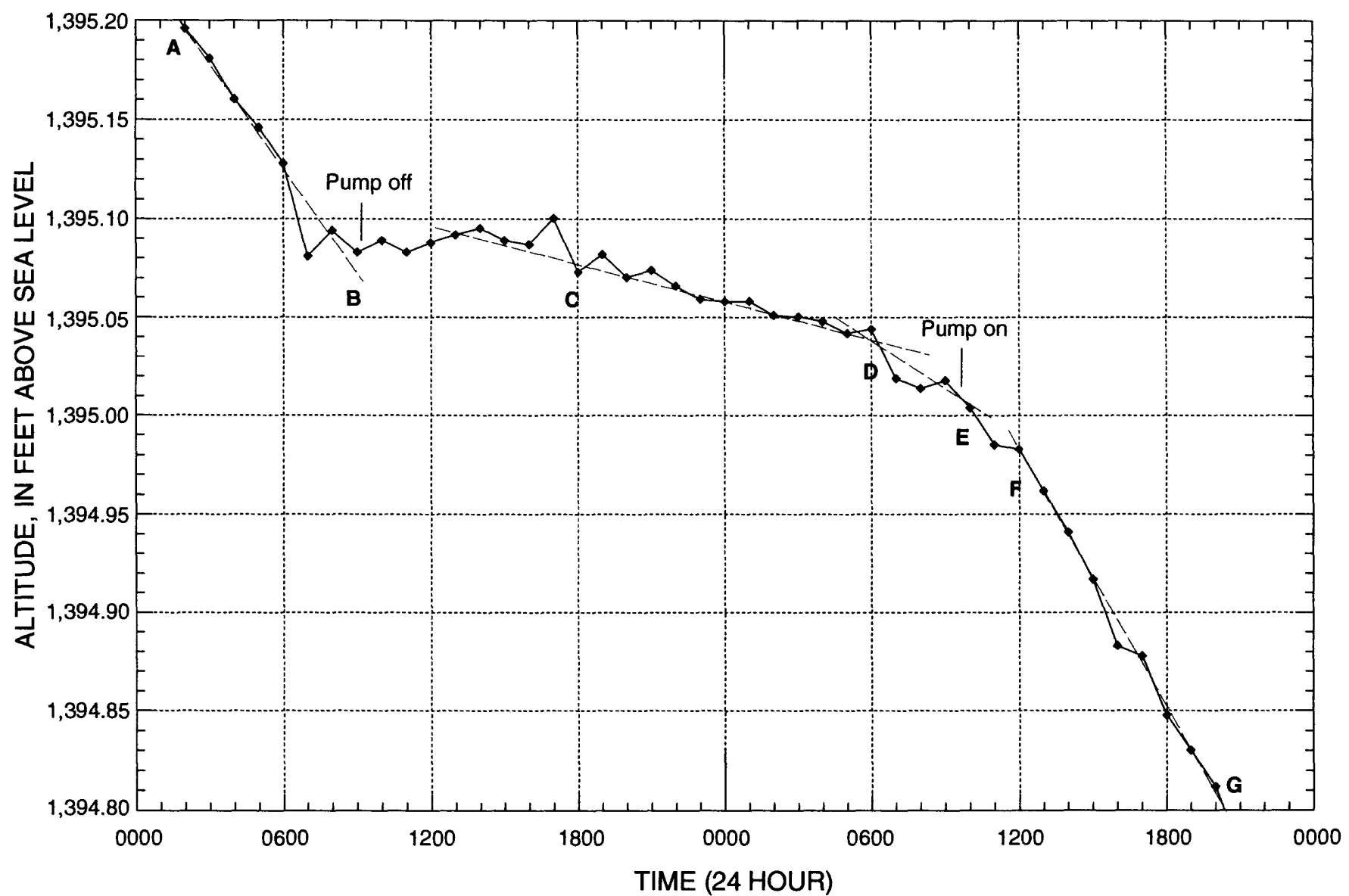

September 11

September 12

Figure 14. Water-level altitudes in Trumble Road pond, September 11-12, 1995.

in the evening and nighttime hours when evaporation presumably is minimized, and because the pan is not sheltered by tall berms (as is the pond). Inclusion of evaporation (from adjusted panevaporation) in the estimate yields a minimum value of infiltration rate of -0.39 acre- $\mathrm{ft} / \mathrm{d}$ $(-140 \mathrm{acre}-\mathrm{ft} / \mathrm{yr})$. This value probably is not valid given the use of 24-hour pan evaporation rate in place of nighttime actual pond evaporation rate. In addition, it should be noted that the rate of infiltration may be different at different pond stages owing to larger or smaller head differentials between the pond surface and the water table.

\section{Winchester Ponds}

Pond B (west pond) at the Winchester pond site (fig. 8C) was instrumented with a Rittmeyer Series MPSDI submersible pressure transducer placed in a 2-inch-diameter stilling well and connected to a Stevens AXSYS SDI-12 data logger, and water-level data were collected for the period June 21-September 6, 1995. Water-level altitudes, shown in figure 15 and table 9 , generally declined steadily during July and August, and the total decline for this period was about $10 \mathrm{ft}$. Treated water was pumped both into and out of the pond on an almost daily basis during the measurement period.

The water-balance equation (eq. 1) was used to estimate infiltration from pond B for July 28-August 19, 1995 (the period with a complete set of inflow/outflow measurements). More water was pumped out (252.7 acre-ft) of the pond than was pumped in (35.28 acre-ft). Water levels in the pond were converted to volumes using a pond-level/pondvolume conversion table provided by EMWD (Alfred Javier, EMWD, written commun., 1996): 


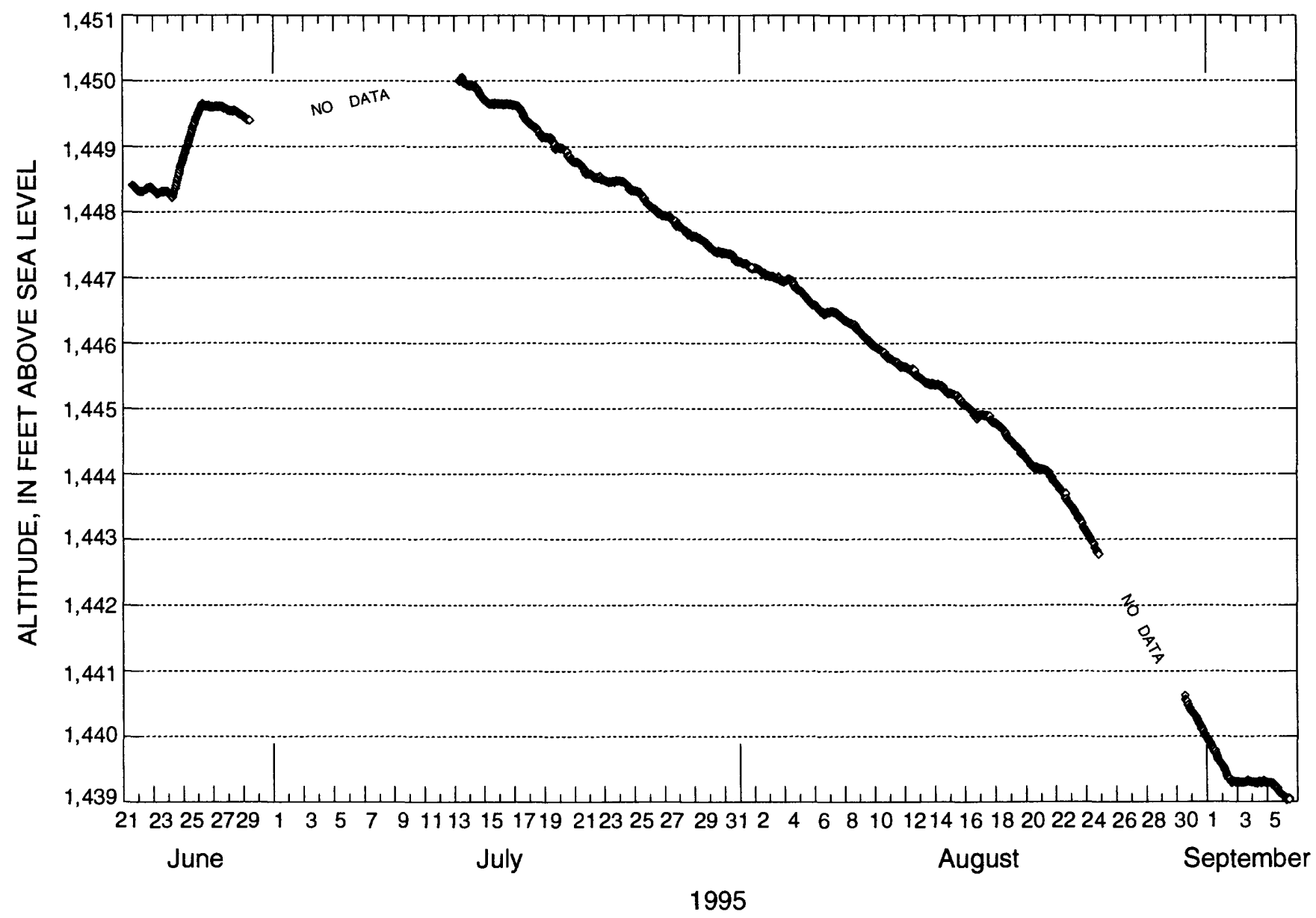

Figure 15. Water-level altitudes in Winchester pond B, June 21 to September 6, 1995.

Pond volume on July $28=130.9 \times 10^{6} \mathrm{gal}=$ $17.50 \times 10^{6} \mathrm{ft}^{3}$, and pond volume on August $19=95.79 \times 10^{6} \mathrm{gal}=$ $12.81 \times 10^{6} \mathrm{ft}^{3}$.

Change in pond volume $=4.69 \times 10^{6} \mathrm{ft}^{3}$.

Evaporation was estimated by multiplying the summed daily pan-evaporation values, measured by MWD at Tucalota Creek near Lake Skinner (about $8 \mathrm{mi}$ south of Winchester), by the appropriate rate of volume change at the prevailing water-level altitude in pond $B$ and by a pan coefficient of 0.7 :

Feet of evaporation $=(6.45 \mathrm{in}) \times(0.7)=$ 4.52 in $=0.377 \mathrm{ft}$;

Rate of pond-volume change at $1,447.7$ to $1,444.4 \mathrm{ft}$ altitude $=\left(4.69 \times 10^{6} \mathrm{ft}^{3}\right) / 3.3 \mathrm{ft}=$ $1.42 \times 10^{6} \mathrm{ft}^{3} / \mathrm{ft}$.

Volume evaporated $=(0.377 \mathrm{ft}) \times(1.42 \times$ $\left.10^{6} \mathrm{ft}^{3} / \mathrm{ft}\right)=0.535 \times 10^{6} \mathrm{ft}^{3}$.
Substitution into equation 1 gives:

Volume infiltrated $=\left[\left(17.50 \times 10^{6} \mathrm{ft}^{3}\right)-\right.$ $\left(12.81 \times 10^{6} \mathrm{ft}^{3}\right]+\left[\left(1.537 \times 10^{6} \mathrm{ft}^{3}\right)-\right.$ $\left.\left(11.07 \times 10^{6} \mathrm{ft}^{3}\right)\right]-\left(0.535 \times 10^{6} \mathrm{ft}^{3}\right)+(0)=$ $-5.38 \times 10^{6} \mathrm{ft}^{3}=-123$ acre-ft.

The average rate of infiltration from pond $\mathrm{B}$ for July 28-August 19 is -5.35 acre- $\mathrm{ft} / \mathrm{d}$ (upward infiltration). As at the Sun City site, the apparent net flow of ground water into the pond may be the result of equilibration of the water-table altitude (which previously had been raised by downward infiltration of pond water and percolation of rainfall) with the altitude of the pond's water level (which had been lowered relatively quickly by netoutward pumping). During the winter months, water-level altitudes in the pond usually are higher than the water table, and downward infiltration is likely to take place. 


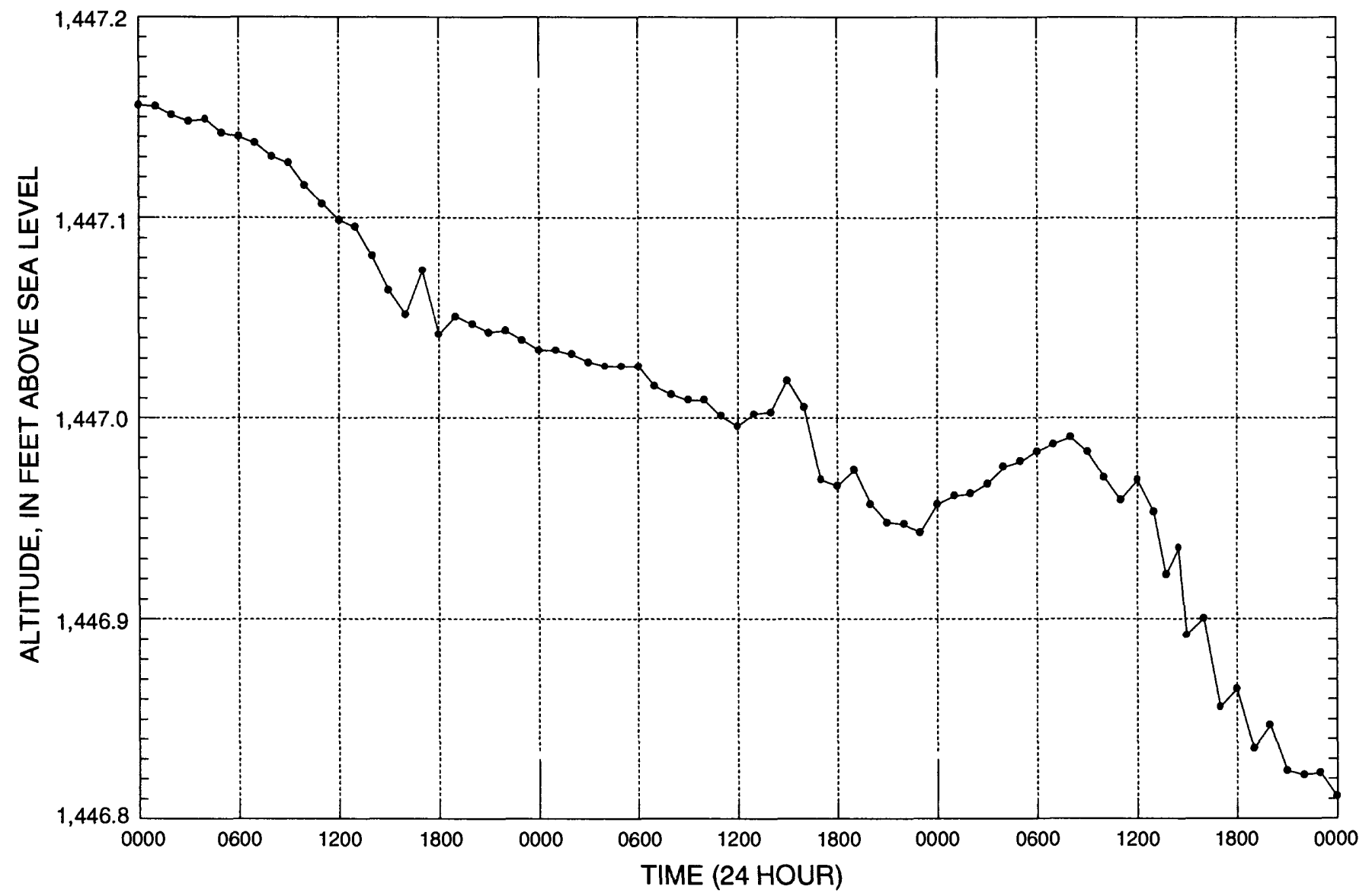

August 2

August 3

August 4

Figure 16. Water-level altitudes in Winchester pond B, August 2-4, 1995.

Records indicate that August 3-4, 1995, was a period of zero measured inflow- or outflowpumping. The exact times that the pumps were turned off and on are not known; but, on the basis of the time of day the meters were read, both pumps were off for a period greater than the span of 1030 on August 3 to 0940 on August 4, 1995. Water-level altitudes in pond B for this 23-hour period (fig. 16) generally declined gradually from 1030 to 2300 on August 3, and then rose gradually until about 0900 on August 4. The early-morning rise may reflect the upward infiltration of ground water into the pond during the nighttime period of reduced evaporation. The average infiltration rate calculated on the basis of data from this 23-hour period, using equation 2 , is 0.75 acre- $\mathrm{ft} / \mathrm{d}$ (downward infiltration).

Comparison of this 1-day value with the longer term infiltration rate for Winchester pond and with infiltration values calculated for the Sun City and Trumble Road sites shows the uncertainties caused by both the use of short-term water-level data and pan-evaporation data. In addition, the values may reflect spatial and temporal variability of infiltration rates. At any of the sites, infiltration rates during times when the ponds are at high stage are likely to be different from the rates estimated above using primarily low-stage data.

Over the longer term (May-September), water levels in shallow observation wells adjacent to the Winchester ponds (fig. 8; table 5) generally reflect the decline in water level in the ponds. During May-September, water levels declined about $11 \mathrm{ft}$ in Pond $\mathrm{B}$ and about $17 \mathrm{ft}$ in Pond A (table 5). The water level in the observation well closest to Pond B (30H3) declined $7.8 \mathrm{ft}$; water levels in wells near Pond A $(30 \mathrm{H} 2,30 \mathrm{H} 1$, and $30 \mathrm{~A} 1)$ declined 7.4 to $11.5 \mathrm{ft}$ (fig. 8C). The water-level altitudes in the closest observation wells, all of which are perforated a minimum of about 9 to $37 \mathrm{ft}$ below the bottom of the ponds, were within less than 1 to $5 \mathrm{ft}$ of the adjacent pond water-level altitudes for the 
dates given in table 5. The magnitude of waterlevel change (decline) is related to distance of the observation well from the ponds. As a comparison, water levels in two unused wells $(5 \mathrm{~S} / 3 \mathrm{~W}-25 \mathrm{~K} 1$ and $5 \mathrm{~S} / 2 \mathrm{~W}-29 \mathrm{~J} 1$ ) located about $1 \mathrm{mi}$ southwest [-25K1] and about $1 \mathrm{mi}$ southeast [-29J1] of the ponds (fig. 1) declined 2.3 and $3.3 \mathrm{ft}$, respectively (table 3).

As the ponds filled in winter, a ground-water mound developed; during summer and autumn, as the pond levels were lowered by pumping, evaporation, and infiltration, the water level in pond $B$ became lower than immediately adjacent groundwater levels. In general, water levels in the observation wells east and south (generally upgradient) of the ponds were a little below the pond water levels during spring (fig. $10 C$; table 5) and early summer, and were a little above the pond water levels during late summer and autumn (fig. $11 C$; table 5). Therefore, seasonal upward infiltration of ground water into pond B occurs primarily from the east side of the pond and, to a lesser extent, the south side. West of the ponds (downgradient), ground-water levels were lower than pond water levels for all the measurement dates; the slightly greater distance of these wells from the ponds also is a factor.

\section{Uncertainties in Infiltration Estimates}

Correctness of the calculated infiltration rate, and possibly of the indicated direction of infiltration, is dependent on the accuracy of the measurements of pumped inflow and outflow, the validity of the panevaporation measurements, and the accuracy of the conversion of pond water-level values to pond volumes. The pumped inflow and outflow values are subject to meter error of as much as 5 percent (Michael Garner, EMWD, oral commun., 1995). Using the Winchester site as an example, 5 percent of the total pumped inflow and outflow for the period of interest for Pond B is 1.8 acre- $\mathrm{ft}$ and 13 acre-ft, respectively. Each pond-volume calculation at the Winchester site has a possible error of about $1.5 \mathrm{acre}-\mathrm{ft}$, and pond-evaporation calculations have a possible error of about 0.2 acre- $\mathrm{ft}$, on the basis of significant figures of the values reported in the pond-water level/pond volume conversion table. The sum of these possible errors is about 17 acre-ft; most of the possible error is associated with the inflow and outflow meters. The possible error in the difference between measured pan evaporation and actual evaporation from the pond surface at sites several miles away, and any error resulting from selection of a pan coefficient of 0.7 , cannot be assessed within the scope of this project.

Possible error for the estimates made in this report for the Sun City and Trumble Road sites is less than that for the Winchester site because inflow and outflow measurements were not used at the Sun City and Trumble Road sites. The possible error instead is primarily related to the pond-water level/pond volume conversion values and errors associated with estimation of actual evaporation.

More reliable estimates of infiltration rates could be obtained by collecting pond water-level data during periods of no pumped inflow or outflow lasting 1 week or more and combining those data with onsite measurements of evaporation. If inflow and outflow pumping cannot be turned off for at least a week, accurate measurement of pumped flows would be a necessity. Understanding of pond infiltration also would be improved through data collection and analysis for all seasons; infiltration rates during times when the ponds are at high stage are likely to be different from the rates estimated using primarily low-stage data because the high stages create substantially greater head differentials between the ponds and the water table. Transferability of infiltration rates could be investigated through data collection and estimation of infiltraton for more than one pond at a site to determine both the variation of rates for different ponds and the effect of infiltration of water from adjacent ponds. The relation between pond levels and ground-water levels could be better defined through the installation of monitoring wells located near the Trumble Road and Sun City ponds and screened a short distance below the water table.

An alternative method of monitoring infiltration from the ponds would involve instrumentation of the unsaturated zone in the vicinity of the ponds to measure moisture content. If an unsaturated zone is present beneath the ponds, position and speed of a downward-moving front could be determined. This information could be used in combination with estimation of change in ground-water storage to determine infiltration rates if the amount of water infiltrating is greater than the error range of the estimated volume of water in storage in the basin.

\section{SUMMARY AND CONCLUSIONS}

During the preliminary phase of a study to determine the quantity and fate of reclaimed water that percolates from storage ponds to the ground- 
water system, 115 wells were located and inventoried in parts of the Perris, Sun City, and Winchester subbasins. Well-construction, historicalwater-quality, and historical-water-level data were compiled for those wells for which data could be obtained. In addition, during the study, water levels were measured in 86 of the wells, and water-levelcontour maps were prepared. The water-level contours indicate flow from the Winchester subbasin northwestward to the south Perris subbasin and southwestward to the Menifee subbasin. From a ground-water divide near the southwest part of the Menifee subbasin, ground water flows north toward the south Perris subbasin and southwestward toward Railroad Canyon Reservoir. In the eastern part of the Menifee subbasin, ground water flows southeastward.

On the basis of information from drillers' logs, three generalized lithologic sections were constructed: from the southwest corner of the Menifee subbasin northeastward through the south Perris subbasin; from the northwest part of the south Perris subbasin southeastward through the Winchester subbasin; and west to east through the Menifee subbasin.

Water-level data were collected from one pond at each of the three storage-pond sites. Water-level data and evaporation data, for periods of no pumped inflow or pumped outflow, were used to estimate an infiltration rate of -0.18 to $-0.15 \mathrm{acre}-\mathrm{ft} / \mathrm{d}$ (upward percolation) for Sun City pond no. 9 during August 3 to September 15, 1995, and a rate of 0.72 acre$\mathrm{ft} / \mathrm{d}$ for Trumble Road pond during September 1112, 1995. For Winchester pond B, a rate of -5.35 acre-ft/d was estimated for July 28 to August 19 , 1995, during which time pumped inflow and outflow were measured. The Trumble Road pond estimate was made on the basis of nighttime measurements and assumes negligible evaporation.

The upward percolation of water in Sun City pond no. 9 and in Winchester pond B probably resulted from the lowering of the water level in the ponds to an altitude below the local water-table altitude, followed by a gradual rise as the pond level equilibrated with the water-table altitude. The range of values and direction of infiltration may be indicative both of the variability of infiltration rates at different sites (and under different conditions) and the possible error ranges of the measured parameters used in calculating infiltration. The parameters subject to the greatest inaccuracy are evaporation and pumped inflow and outflow. Conversion from pond stage to pond volume, and selection of a proper pan coefficient also are possible sources of error. At any of the sites, infiltration rates during times when the ponds are at high stage are likely to be different from the rates estimated using primarily low-stage data.

More reliable estimates of infiltration rates could be obtained by collecting pond water-level data during periods of no pumped inflow or outflow lasting 1 week or more and combining those data with onsite measurements of evaporation. If inflow and outflow pumping cannot be turned off for at least a week, accurate measurement of pumped flows would be a necessity.

\section{References Cited}

Biehler, Shawn and Lee, T.C., 1994, Subsurface structure of the Winchester basin, Riverside County, California: Riverside, Calif., Department of Earth Sciences, University of California, $47 \mathrm{p}$.

Biehler, Shawn and Lee, T.C., 1995, Subsurface structure of the Menifee basin, Riverside County, California: Riverside, Calif., Department of Earth Sciences, University of California, $59 \mathrm{p}$.

California Department of Water Resources, 1959, Santa Ana River investigation: California Department of Water Resources Bulletin No. 15, 207 p.

California Department of Water Resources, 1978, Water resources evaluation of the San Jacinto area: California Department of Water Resources District Report, $81 \mathrm{p}$.

California Department of Water Resources, 1979, Ground water quality conditions in Menifee, Winchester, and South Perris subareas: California Department of Water Resources District Report, $82 \mathrm{p}$.

Field, J.A., Barber, L.B., II, Thurman, E. M., Moore, B.L., Lawrence, D.L., and Peake, D.A., 1992a, Fate of alkylbenzenesulfonates and dialkyltetralinsulfonates in sewage-contaminated groundwater: Environmental Science and Technology, v. 26, p. 1140-1148.

Field J.A., Leenheer, J.A., Thorn, K.A., Barber, L.B, II, Rostad, C., Macalady, D.L., and Daniel, S.R., $1992 \mathrm{~b}$, Identification of persistent anionic surfactant-derived chemicals in sewage effluent and groundwater: Journal of Contaminant Hydrology, v. 9, p. 55-78.

Lang, D.J., 1979, Water-resources data, 1970-75, for Perris Valley and vicinity, Riverside County, California: U.S. Geological Survey Open-File Report 79-1256, 127 p. 
NBS/Lowry, 1987, Feasibility of well recharge of reclaimed water and well extraction of groundwater for Menifee Village irrigation: Irvine, Calif., NBS/Lowry, Engineers and Planners.

Pruitt, J.B., Elder, J.F., and Johnson, I.K., 1988, Effects of treated municipal effluent irrigation on ground water beneath sprayfields, Tallahassee, Florida: U.S. Geological Survey Open-File Report 884092, $35 \mathrm{p}$.

Rees, T.F., Bright, D.J., Fay, R.G., Christensen, A.H., Anders, R., Baharie, B.S., and Land, M.T., 1994 [1995], Geohydrology, water quality, and nitrogen geochemistry in the saturated and unsaturated zones beneath various land uses, Riverside and San Bernardino Counties, California, 1991-93: U.S. Geological Survey Water-Resources Investigations Report 94-4127, $267 \mathrm{p}$.
Schneider, B.J., Ku, H.F.H., and Oaksford, E.T., 1987, Hydrologic effects of artificial-recharge experiments with reclaimed water at East Meadow, Long Island, New York: U.S. Geological Survey Water-Resources Investigations Report 85-4323, 79 p.

Sylvester, M.A., 1983, Land application of wastewater and its effect on ground-water quality in the Livermore-Amador Valley, Alameda County, California: U.S. Geological Survey WaterResources Investigation Report 82-4100, 53 p., 2 pls.

Veihmeyer, F.J., 1964, Chapter 11--Evapotranspiration, in Chow, V. T., ed., Handbook of Applied Hydrology: New York, McGraw-Hill Book Company, p. 11-1 to 11-38. 


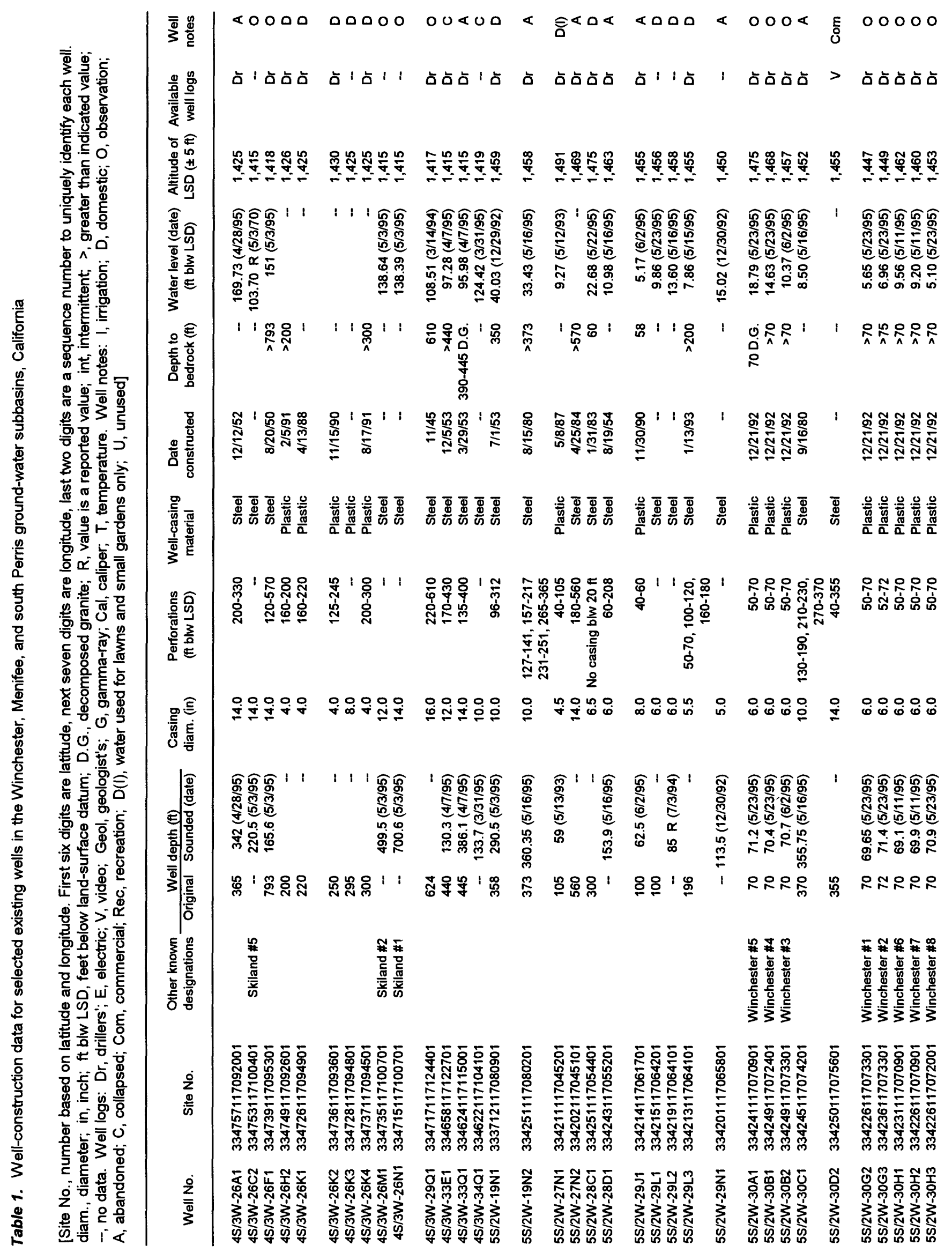




\begin{tabular}{|c|c|c|c|c|c|c|c|c|c|}
\hline 弯 & $\ll$ & $\ll \Delta \Delta \ll$ & ००००० & -0000 & $0-\ll<0$ & --00 & -๑০০ฮิ & ฮัด ०-- & $0-$ \\
\hline 产 & $\grave{a}$ & 方 & 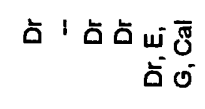 & 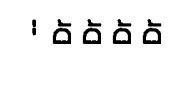 & 5 & '方方 & |' | & 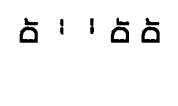 & ' \\
\hline 焉 & $\stackrel{\bar{g}}{-}$ & 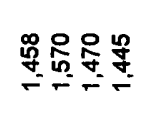 & 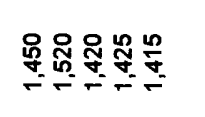 & 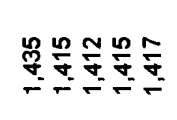 & ঙ্ণি & 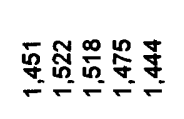 & 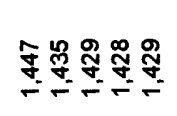 & 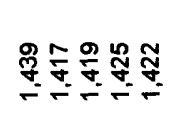 & 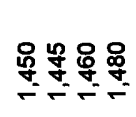 \\
\hline 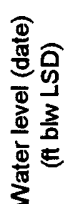 & 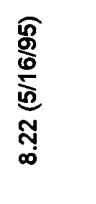 & 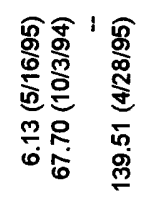 & 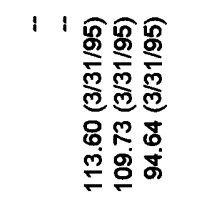 & 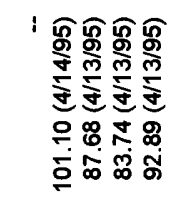 & 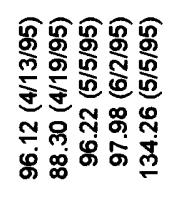 & 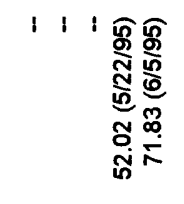 & 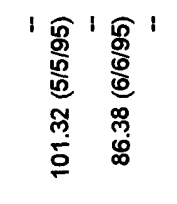 & 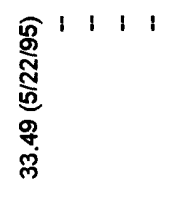 & 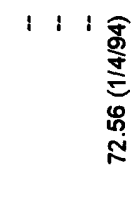 \\
\hline 送 & 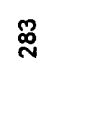 & $\underset{N}{\stackrel{R}{N}-\varphi}$ & " & ' & 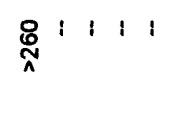 & 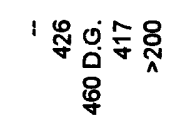 & 1 : & 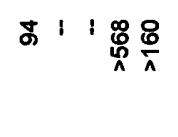 & 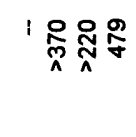 \\
\hline 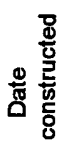 & $\underset{\stackrel{\circ}{N}}{\stackrel{\text { N }}{N}}$ & 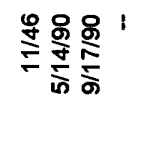 & 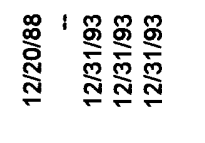 & 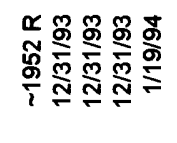 & 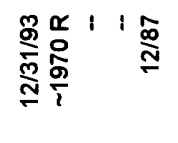 & ' & 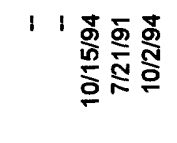 & 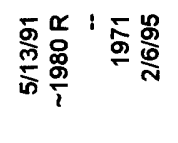 & 哭' \\
\hline $\bar{\sigma}$ & $\frac{\bar{\Phi}}{\bar{\omega}}$ & 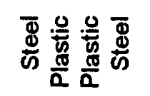 & 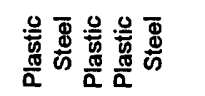 & 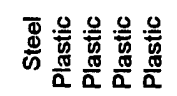 & 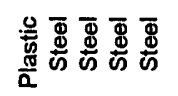 & 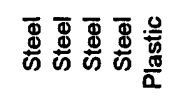 & 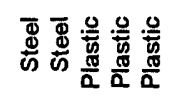 & 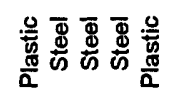 & 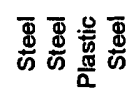 \\
\hline 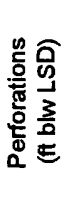 & 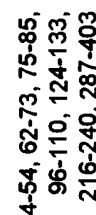 & 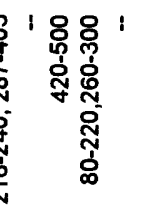 & 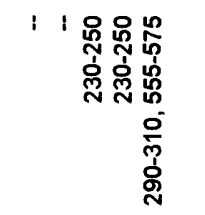 & 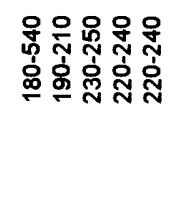 & 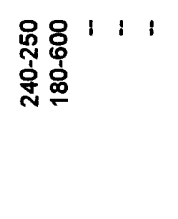 & ' & 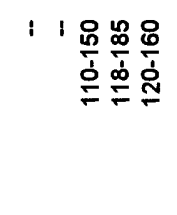 & 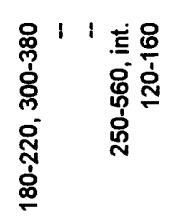 & 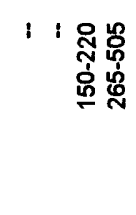 \\
\hline$\Xi$ & $\stackrel{\circ}{\stackrel{丶}{ }}$ & 욲 우⼴ & 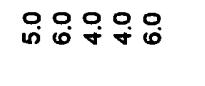 & 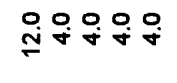 & 吠哭怘 10 & 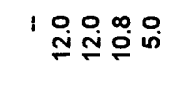 & 10000 & 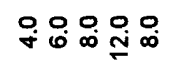 & 电 \\
\hline 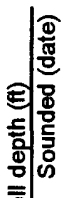 & 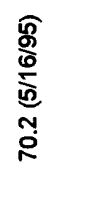 & 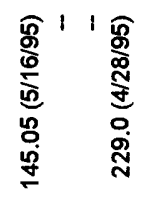 & 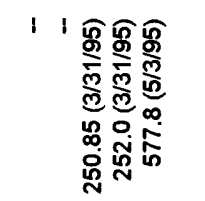 & 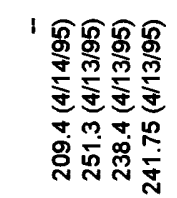 & 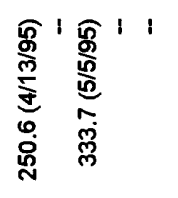 & 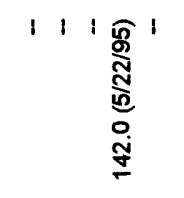 & 11111 & 11111 & $1: 1: 1$ \\
\hline $3\left|\begin{array}{c}\overline{0} \\
3\end{array}\right| \overline{\underline{\sigma}}$ & $\stackrel{\infty}{\%}$ & 오윰용요 & 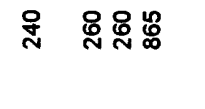 & 家 : & 足㤂' ' స̃ & 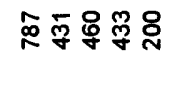 & 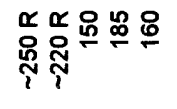 & 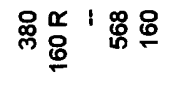 & 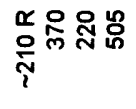 \\
\hline 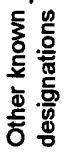 & & & 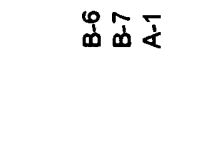 & 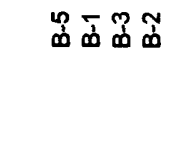 & Фా & & & & \\
\hline $\begin{array}{l}\sum_{0}^{0} \\
\dot{0} \\
i=5\end{array}$ & 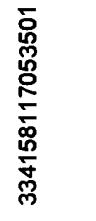 & 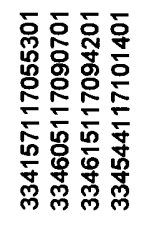 & 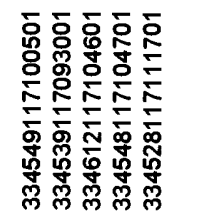 & 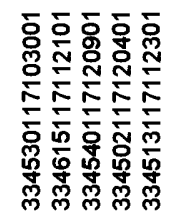 & 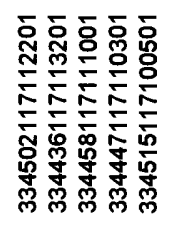 & 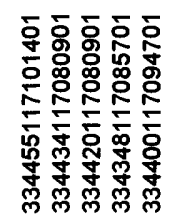 & 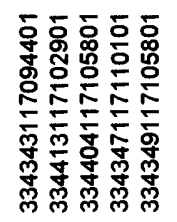 & 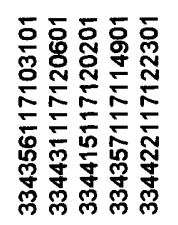 & 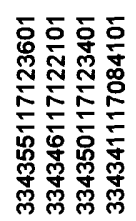 \\
\hline$\frac{2}{\bar{a}}$ & 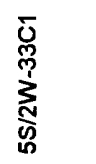 & 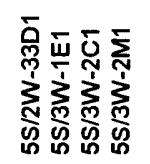 & 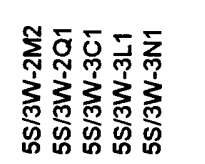 & 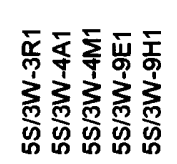 & 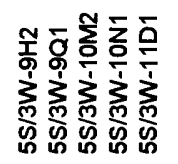 & 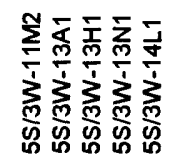 & 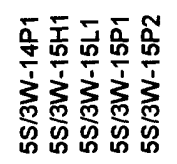 & 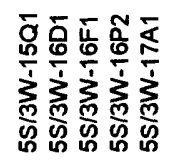 & 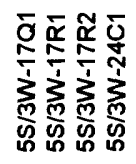 \\
\hline
\end{tabular}




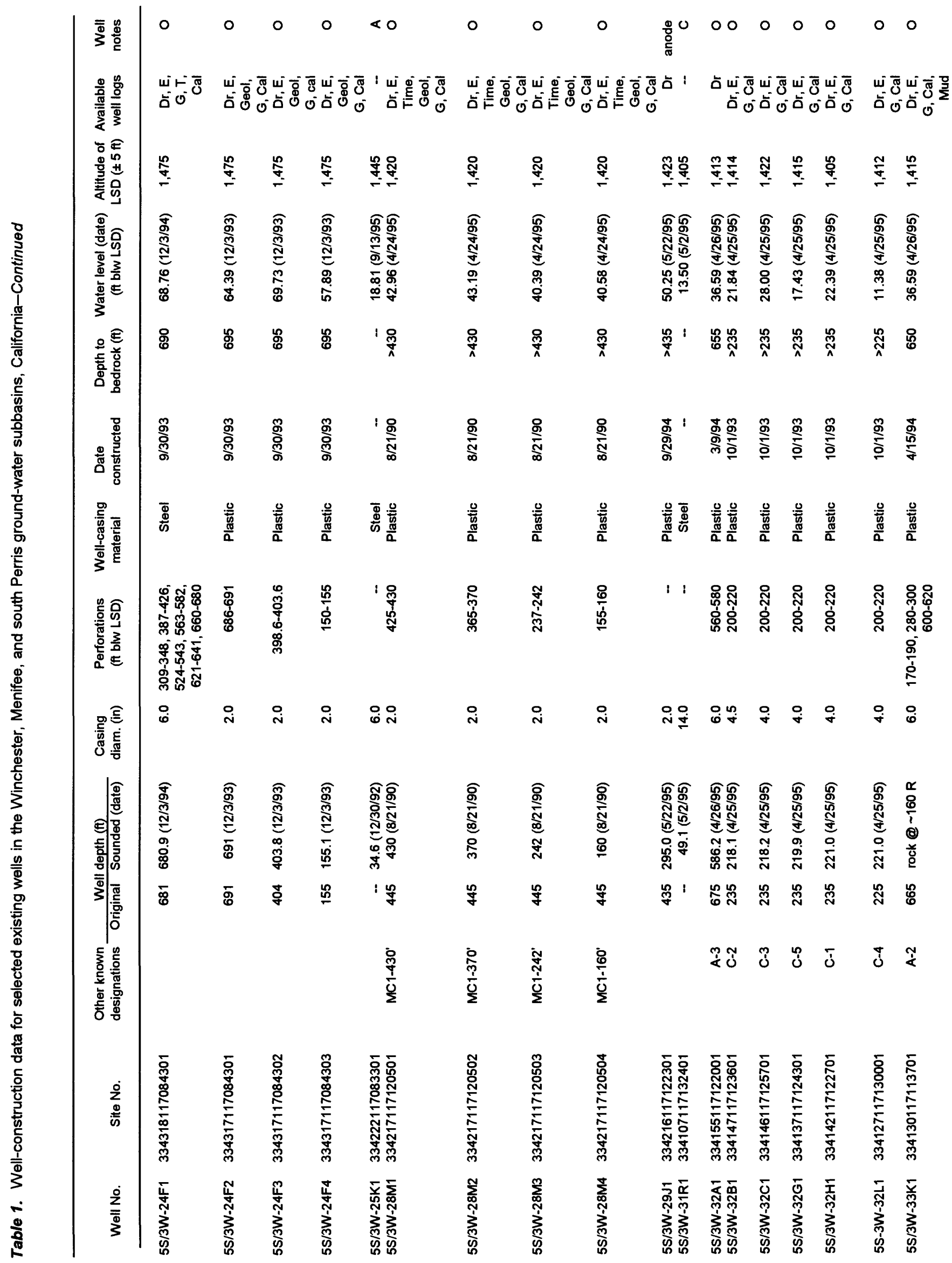




\begin{tabular}{|c|c|c|c|c|c|c|c|c|c|c|c|c|}
\hline 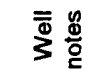 & $\varangle 0 \quad 0$ & 0000 & כ & $\ll<\underset{\mathscr{\alpha}}{\mathbb{\alpha}} 00$ & $\circ$ & 0 & $\underset{\mathscr{Q}}{\mathscr{\mathscr { O }}}$ & $\underset{\mathscr{Q}}{\mathscr{8}}$ & $\underset{\mathscr{\alpha}}{\mathscr{8}}$ & $\stackrel{8}{\longleftarrow}$ & $\varangle$ & ४०० \\
\hline 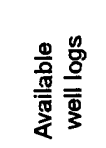 & 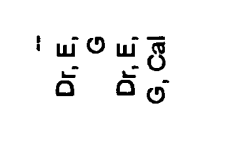 & | & ¿ & U: 1: & 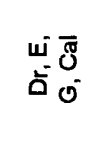 & 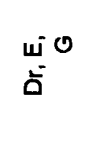 & 出家 & 嵌 & 㟧 & i̊ & ¿ & 11 \\
\hline 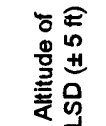 & 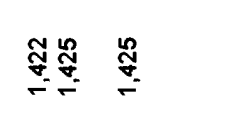 & 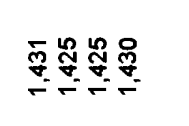 & 糫 & 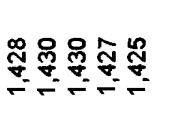 & $\stackrel{\mathscr{q}}{\stackrel{q}{*}}$ & $\stackrel{\mathscr{q}}{\stackrel{\leftrightarrow}{-}}$ & 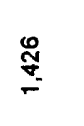 & $\stackrel{\infty}{\underset{\sim}{*}}$ & $\stackrel{\infty}{\stackrel{\sim}{*}}$ & $\stackrel{\infty}{\underset{\sim}{\sim}}$ & $\stackrel{\mathscr{q}}{\stackrel{q}{*}}$ & 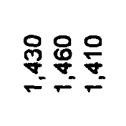 \\
\hline 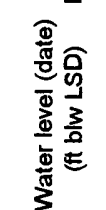 & 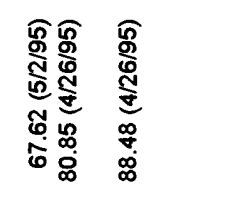 & 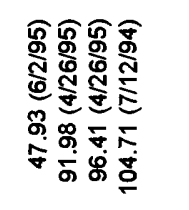 & 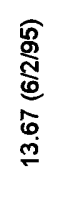 & 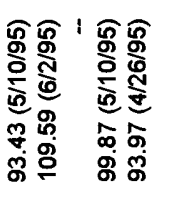 & 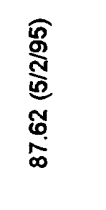 & 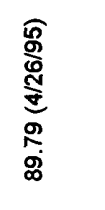 & 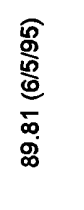 & 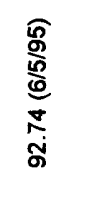 & 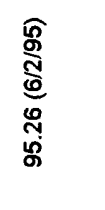 & 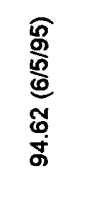 & 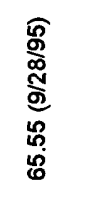 & 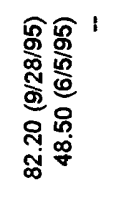 \\
\hline 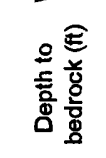 & '吕 & 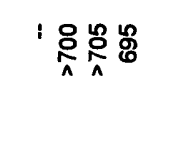 & $\underline{\underline{g}}$ & $111: 8$ & $\stackrel{\mathscr{g}}{\circ}$ & 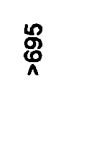 & 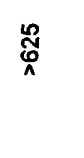 & : & $\begin{array}{l}\dot{0} \\
\dot{0} \\
0 \\
0\end{array}$ & & ఫ & $: 1$ \\
\hline 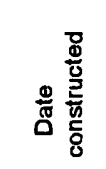 & ' & 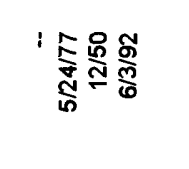 & 商 & : : : & 粱 & 兽 & 离 & $\frac{\infty}{\frac{\infty}{5}}$ & 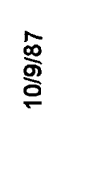 & 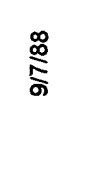 & 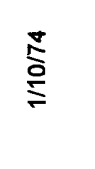 & i \\
\hline 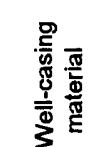 & 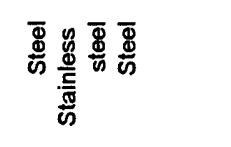 & 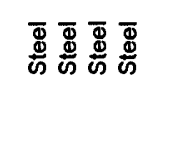 & $\overline{\bar{\Phi}}$ & 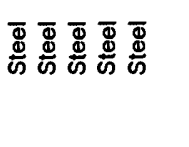 & $\frac{\bar{\Phi}}{\bar{n}}$ & $\frac{\bar{\Phi}}{\omega}$ & $\stackrel{\bar{\Phi}}{\grave{\omega}}$ & $\frac{\bar{\Phi}}{\bar{\omega}}$ & $\stackrel{\bar{\Phi}}{\tilde{\omega}}$ & 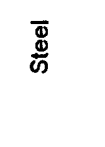 & $\frac{\overline{\mathrm{g}}}{\frac{\mathrm{w}}{\omega}}$ & 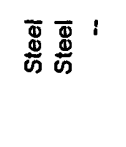 \\
\hline 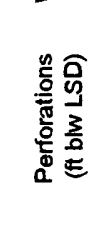 & 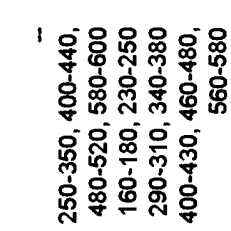 & 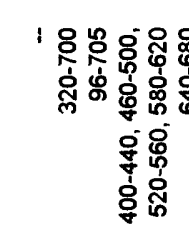 & & 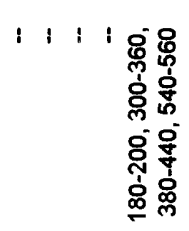 & & 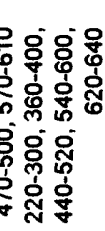 & 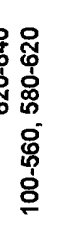 & ' & ! & ' & 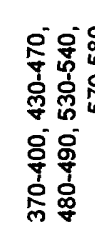 & \\
\hline 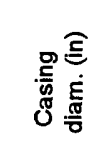 & 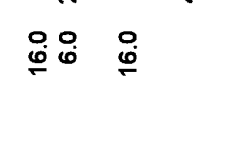 & 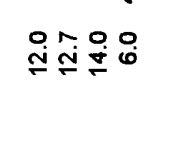 & $\ddot{\circ}$ & 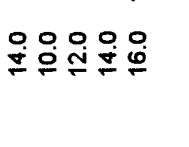 & $\stackrel{\circ}{\circ}$ & $\stackrel{\circ}{\circ}$ & $\stackrel{\circ}{\circ}$ & $\stackrel{\circ}{\circ}$ & $\stackrel{\circ}{\circ}$ & ' & $\stackrel{q}{\ddagger}$ & 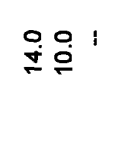 \\
\hline 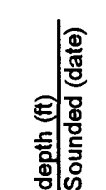 & 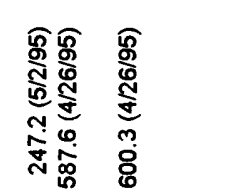 & 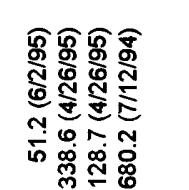 & & 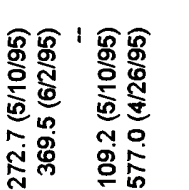 & 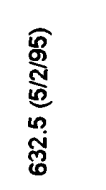 & 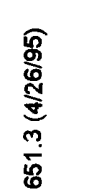 & : & ' & ! & & 1 & 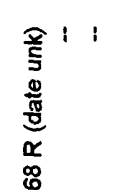 \\
\hline 3 & ! 足 吕 & 옷옷몽온 & 움 & : : : ৪ : \& & $\mathscr{\mathscr { E }}$ & $\stackrel{\mathscr{g}}{\mathscr{g}}$ & $\mathscr{\sigma}$ & สี & 吕 & $\frac{5}{5}$ & ¿ & 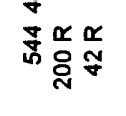 \\
\hline 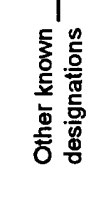 & 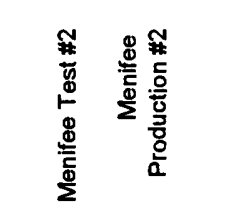 & 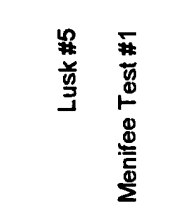 & & 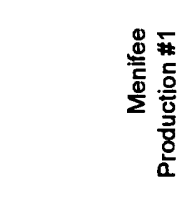 & 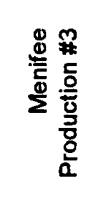 & 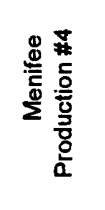 & & & & 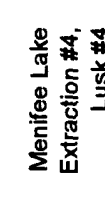 & & \\
\hline $\begin{array}{l}\frac{\partial}{2} \\
\frac{0}{\bar{\omega}}\end{array}$ & 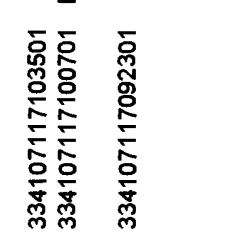 & 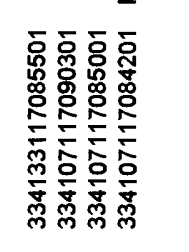 & & 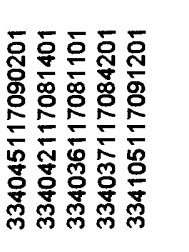 & 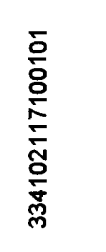 & 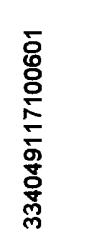 & 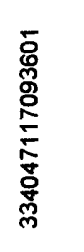 & 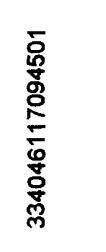 & 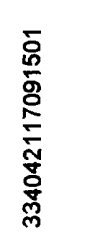 & 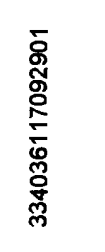 & 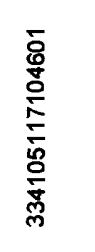 & 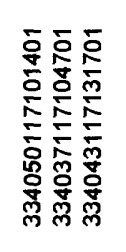 \\
\hline$\frac{\dot{s}}{\frac{2}{\bar{s}}}$ & 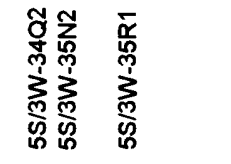 & 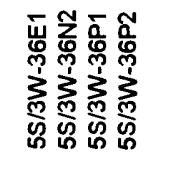 & 品 & 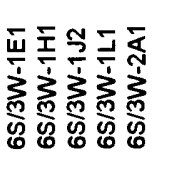 & $\begin{array}{l}\overline{\hat{N}} \\
\text { 商 } \\
\text { con }\end{array}$ & 离 & 突 & 蒿 & 弪 & 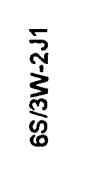 & $\begin{array}{l}\overline{0} \\
\text { 商 } \\
\frac{10}{6}\end{array}$ & 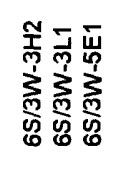 \\
\hline
\end{tabular}


Table 2. Historical water-quality data for selected wells in the Winchester, Menifee, and south Perris ground-water subbasins, California

[wells not shown in figure 1 were not located in the field or have been destroyed; $\mathrm{ft}$, foot; $\mathrm{ft}$ blw LSD, feet below land-surface datum; $\mu \mathrm{S} / \mathrm{cm}$, microsiemens per centimeter at $25^{\circ} \mathrm{C} ;{ }^{\circ} \mathrm{C}$, degrees Celsius; $\mathrm{mg} / \mathrm{L}$, milligrams per liter; $\mu \mathrm{g} / \mathrm{L}$, micrograms per liter; unsat., unsaturated; RCFCD, Riverside County Flood Control and Water Conservation District; EMWD, Eastern Municipal Water District; DPH, California Department of Public Health; DWR, California Department of Water Resouces; WA, Western Analytical Laboratories; Babcock, E.S. Babcock and Sons, Inc.; WNTL, Watercheck National Testing Laboratories; asterisk( $\left(^{*}\right)$ indicates additional data are available; $<$, less than; --, no data]

\begin{tabular}{|c|c|c|c|c|c|c|c|c|}
\hline \multirow[t]{2}{*}{ Well No. } & \multirow[t]{2}{*}{ Date } & \multirow{2}{*}{$\begin{array}{l}\text { Water level } \\
\text { (ft blw LSD) }\end{array}$} & \multirow{2}{*}{$\begin{array}{c}\text { Well depth } \\
\text { (ft) }\end{array}$} & \multirow{2}{*}{$\begin{array}{c}\text { Altitude } \\
\text { of LSD (ft) }\end{array}$} & \multicolumn{2}{|c|}{ Specific conductance $(\mu \mathrm{S} / \mathrm{cm})$} & \multirow{2}{*}{$\begin{array}{c}\text { pH (lab) } \\
\text { (Std. units) } \\
\end{array}$} & \multirow{2}{*}{$\begin{array}{c}\text { Water } \\
\text { temperature } \\
\left({ }^{\circ} \mathrm{C}\right)\end{array}$} \\
\hline & & & & & Fieid & Lab & & \\
\hline \multirow[t]{8}{*}{$4 S / 3 W-26 C 2$} & $8 / 26 / 88$ & -- & 220.5 & 1,415 & -- & 5,450 & 7.4 & - \\
\hline & $1 / 20 / 89$ & -- & & & - & 6,800 & 8.1 & -- \\
\hline & $4 / 18 / 89$ & - & & & -- & 4,100 & 8.3 & - \\
\hline & $4 / 20 / 89$ & - & & & - & 5,000 & 7.6 & -- \\
\hline & $7 / 6 / 89$ & -- & & & -- & 4,350 & 8.2 & -- \\
\hline & $10 / 12 / 89$ & - & & & - & 5,200 & 8.7 & -- \\
\hline & $12 / 26 / 89$ & -- & & & -- & 4,750 & 8.8 & - \\
\hline & $7 / 14 / 92$ & -- & & & -- & 4,900 & 7.4 & -- \\
\hline \multirow[t]{5}{*}{$4 S / 3 W-26 F 1$} & $10 / 29 / 69$ & -- & 165.6 & 1,418 & -- & 7,180 & 7.7 & 24.5 \\
\hline & $4 / 16 / 70$ & - & & & -- & 7,170 & 7.1 & 23.5 \\
\hline & $5 / 11 / 71$ & - & & & - & 8,660 & 7.8 & 25.5 \\
\hline & $8 / 26 / 88$ & - & & & -- & 4,620 & 7.7 & - \\
\hline & $1 / 20 / 89$ & - & & & -- & - & -- & - \\
\hline \multirow[t]{8}{*}{ 4S/3W-26J1 } & $5 / 8 / 58$ & - & 470 & 1,445 & -- & 1,390 & 7.6 & 24.5 \\
\hline & $10 / 14 / 58$ & -- & & & -- & 1,720 & 7.3 & 26.5 \\
\hline & 3/31/59 & -- & & & -- & 1,660 & 8.0 & 26.0 \\
\hline & $4 / 28 / 69$ & - & & & -- & 1,990 & 7.7 & 26.5 \\
\hline & $11 / 20 / 70$ & -- & & & -- & 2,150 & 7.9 & 26.5 \\
\hline & $5 / 11 / 71$ & -- & & & - & 1,730 & 7.9 & 28.0 \\
\hline & $5 / 17 / 72$ & -- & & & -- & 2,440 & 7.9 & 26.5 \\
\hline & $5 / 2 / 73$ & -- & & & -- & 2,990 & 7.7 & 26.5 \\
\hline \multirow[t]{2}{*}{$4 S / 3 W-26 K 3$} & $5 / 23 / 89$ & - & 295 & 1,425 & - & 11,600 & -- & - \\
\hline & $11 / 20 / 91$ & - & & & -- & 16,200 & 7.2 & - \\
\hline \multirow[t]{10}{*}{$4 S / 3 W-26 M 1$} & $8 / 26 / 88$ & - & 499.5 & 1,415 & -- & 2,860 & 8.5 & - \\
\hline & $9 / 16 / 88$ & - & & & -- & 2,940 & 8.6 & - \\
\hline & $1 / 12 / 89$ & - & & & -- & 3,000 & 7.9 & - \\
\hline & $4 / 20 / 89$ & - & & & - & 1,680 & 8.2 & - \\
\hline & $7 / 11 / 89$ & - & & & -- & 2,200 & 8.2 & -- \\
\hline & $10 / 11 / 89$ & - & & & -- & - & -- & - \\
\hline & $12 / 20 / 89$ & - & & & -- & 2,600 & 7.8 & - \\
\hline & $8 / 8 / 90$ & - & & & -- & 2,090 & 8.1 & - \\
\hline & $6 / 10 / 91$ & - & & & -- & - & -- & -- \\
\hline & $7 / 9 / 92$ & -- & & & -- & 1,100 & 8.5 & - \\
\hline \multirow[t]{8}{*}{$4 S / 3 W-26 N 1$} & $8 / 26 / 88$ & - & 700.6 & 1,415 & - & 9,680 & 8.7 & - \\
\hline & $9 / 16 / 88$ & - & & & -- & 9,460 & 6.8 & - \\
\hline & $12 / 29 / 88$ & -- & & & - & 29,000 & 7.6 & -- \\
\hline & $4 / 21 / 89$ & -- & & & -- & 17,500 & 7.4 & -- \\
\hline & $7 / 12 / 89$ & -- & & & - & 17,000 & 7.4 & -- \\
\hline & $10 / 10 / 89$ & -- & & & - & 30,000 & 7.6 & - \\
\hline & $1 / 24 / 91$ & -- & & & -- & 23,000 & 7.1 & - \\
\hline & $7 / 7 / 92$ & -- & & & -- & 13,000 & 7.2 & -- \\
\hline \multirow[t]{4}{*}{$4 S / 3 W-28 C 1$} & $3 / 15 / 54$ & - & 300 & 1,421 & -- & 2,310 & 7.7 & 20.0 \\
\hline & $8 / 9 / 55$ & - & & & - & - & -- & 21.0 \\
\hline & $10 / 16 / 58$ & - & & & - & 1,900 & 7.7 & 22.0 \\
\hline & $3 / 31 / 59$ & - & & & - & 1,810 & 7.4 & 22.0 \\
\hline \multirow[t]{3}{*}{$4 \mathrm{~S} / 3 \mathrm{~W}-28 \mathrm{H} 1$} & $5 / 3 / 65$ & - & 42 & 1,419 & - & 2,800 & 7.5 & -- \\
\hline & $7 / 19 / 68$ & - & & & -- & 2,860 & 7.6 & - \\
\hline & $10 / 17 / 68$ & -- & & & -- & 3,500 & 7.8 & 30.0 \\
\hline $4 S / 3 W-29 C 3$ & $4 / 19 / 77$ & -- & - & - & -- & 1,800 & 7.0 & - \\
\hline $4 S / 3 W-29 F 3$ & $4 / 19 / 77$ & -- & -- & - & - & 1,730 & 7.1 & - \\
\hline \multirow[t]{2}{*}{$4 S / 3 W-29 G 2$} & $4 / 16 / 70$ & - & 595 & 1,420 & -- & 2,450 & 7.4 & 21.0 \\
\hline & $5 / 11 / 71$ & -- & & & -- & 2,420 & 8.1 & 22.0 \\
\hline
\end{tabular}


Table 2. Historical water-quality data for selected wells in the Winchester, Menifee, and south Perris ground-water subbasins, California-Continued

\begin{tabular}{|c|c|c|c|c|c|c|c|c|}
\hline \multirow[t]{2}{*}{ Well No. } & \multirow{2}{*}{$\begin{array}{c}\text { Hardness } \\
\left(\mathrm{mg} / \mathrm{L} \text { as } \mathrm{CaCO}_{3}\right) \\
\end{array}$} & \multirow{2}{*}{$\begin{array}{c}\text { Calcium, } \\
\text { dissolved } \\
(\mathrm{mg} / \mathrm{L})\end{array}$} & \multirow{2}{*}{$\begin{array}{c}\text { Magnesium, } \\
\text { dissolved } \\
(\mathrm{mg} / \mathrm{L})\end{array}$} & \multirow{2}{*}{$\begin{array}{c}\text { Sodium, } \\
\text { dissolved } \\
(\mathrm{mg} / \mathrm{L})\end{array}$} & \multirow{2}{*}{$\begin{array}{c}\text { Potassium, } \\
\text { dissolved } \\
\text { (mg/L as K) }\end{array}$} & \multirow{2}{*}{$\begin{array}{c}\text { Bicarbonate } \\
\left(\mathrm{mg} / \mathrm{L} \text { as } \mathrm{HCO}_{3}\right)\end{array}$} & \multicolumn{2}{|c|}{ Alkalinity $\left(\mathrm{mg} / \mathrm{L}\right.$ as $\left.\mathrm{CaCO}_{3}\right)$} \\
\hline & & & & & & & Field & Lab \\
\hline \multirow[t]{6}{*}{$4 S / 3 W-26 C 2$} & 690 & 260 & 9.0 & 730.0 & 10 & 24 & - & 20 \\
\hline & 1,300 & 450 & 20.0 & 860.0 & 10 & 58 & - & - \\
\hline & 770 & 220 & 4.5 & 690.0 & 8 & 23 & - & -- \\
\hline & 680 & 260 & 6.0 & 640.0 & 7 & 15 & - & -- \\
\hline & 600 & 240 & 2.8 & 600.0 & 8 & 15 & - & -- \\
\hline & 750 & 200 & 60.0 & 800.0 & 3 & 230 & - & 190 \\
\hline \multirow[t]{3}{*}{$4 S / 3 W-26 F 1$} & 2,300 & 670 & 150.0 & 740.0 & 16 & 79 & - & 65 \\
\hline & 2,100 & 670 & 110.0 & 660.0 & 20 & 91 & - & 75 \\
\hline & 2,300 & 740 & 98.0 & 830.0 & 13 & 58 & -- & 58 \\
\hline \multirow{6}{*}{$4 \mathrm{~S} / 3 \mathrm{~W}-26 \mathrm{~J} 1$} & 370 & 110 & 24.0 & 170.0 & - & 67 & -- & -- \\
\hline & 230 & 84 & 4.0 & 280.0 & 6 & 70 & -- & 57 \\
\hline & 270 & 110 & 2.9 & 290.0 & 5 & 63 & - & 52 \\
\hline & 190 & 68 & 3.6 & 270.0 & 4 & 45 & - & 45 \\
\hline & 330 & 130 & 5.6 & 340.0 & 5 & 62 & - & 51 \\
\hline & 418 & 160 & 5.6 & 420.0 & 7 & 60 & - & 50 \\
\hline \multirow[t]{2}{*}{$4 S / 3 W-26 K 3$} & 6,000 & 2,120 & 160.0 & 1260.0 & 29 & 31 & - & 25 \\
\hline & - & -- & - & - & - & -- & - & -- \\
\hline \multirow[t]{4}{*}{$4 S / 3 W-26 M 1$} & 400 & 150 & 7.0 & 400.0 & 6 & 15 & - & 33 \\
\hline & 520 & 190 & 10.0 & 400.0 & 6 & 12 & - & 25 \\
\hline & 540 & 200 & 8.0 & 370.0 & 4 & 46 & - & - \\
\hline & 150 & 54 & 2.4 & 270.0 & 2 & 66 & - & -- \\
\hline \multirow{6}{*}{$4 S / 3 W-26 N 1$} & 7,100 & 2,500 & 95.0 & 2620.0 & 29 & 19 & - & -- \\
\hline & 3,800 & 1,400 & 49.0 & 1440.0 & 16 & 50 & - & -- \\
\hline & 4,200 & 1,580 & 58.0 & 1500.0 & 18 & 41 & - & - \\
\hline & 6,000 & 2,220 & 100.0 & 2800.0 & 35 & 13 & - & - \\
\hline & 7,600 & 2,850 & 120.0 & 2600.0 & 44 & 61 & - & 50 \\
\hline & 3,580 & 1,340 & 54.0 & 1360.0 & 18 & 43 & - & 35 \\
\hline \multirow[t]{4}{*}{$4 S / 3 W-28 C 1$} & - & 260 & 66.0 & 120.0 & 7 & 100 & - & - \\
\hline & -- & 210 & 42.0 & 120.0 & 6 & 110 & - & - \\
\hline & 700 & 190 & 53.0 & 110.0 & -- & 130 & - & -- \\
\hline & 610 & 170 & 46.0 & 100.0 & - & 130 & - & - \\
\hline \multirow[t]{3}{*}{$4 S / 3 W-28 H 1$} & 1,100 & 340 & 62.0 & 240.0 & 4 & 330 & - & -- \\
\hline & 960 & 240 & 87.0 & 250.0 & 3 & 230 & - & 190 \\
\hline & 1,200 & 290 & 120.0 & 220.0 & 5 & 290 & -- & 230 \\
\hline $4 S / 3 W-29 C 3$ & 650 & 160 & 62.0 & 120.0 & 3 & 210 & - & -- \\
\hline $4 S / 3 W-29 F 3$ & 650 & 170 & 57.0 & 130.0 & 6 & 230 & - & - \\
\hline \multirow[t]{2}{*}{$4 S / 3 W-29 G 2$} & 600 & 190 & 31.0 & 230.0 & 5 & 96 & - & 79 \\
\hline & 610 & 180 & 37.0 & 240.0 & 6 & 79 & - & 79 \\
\hline
\end{tabular}


Table 2. Historical water-quality data for selected wells in the Winchester, Menifee, and south Perris ground-water subbasins, California--Continued

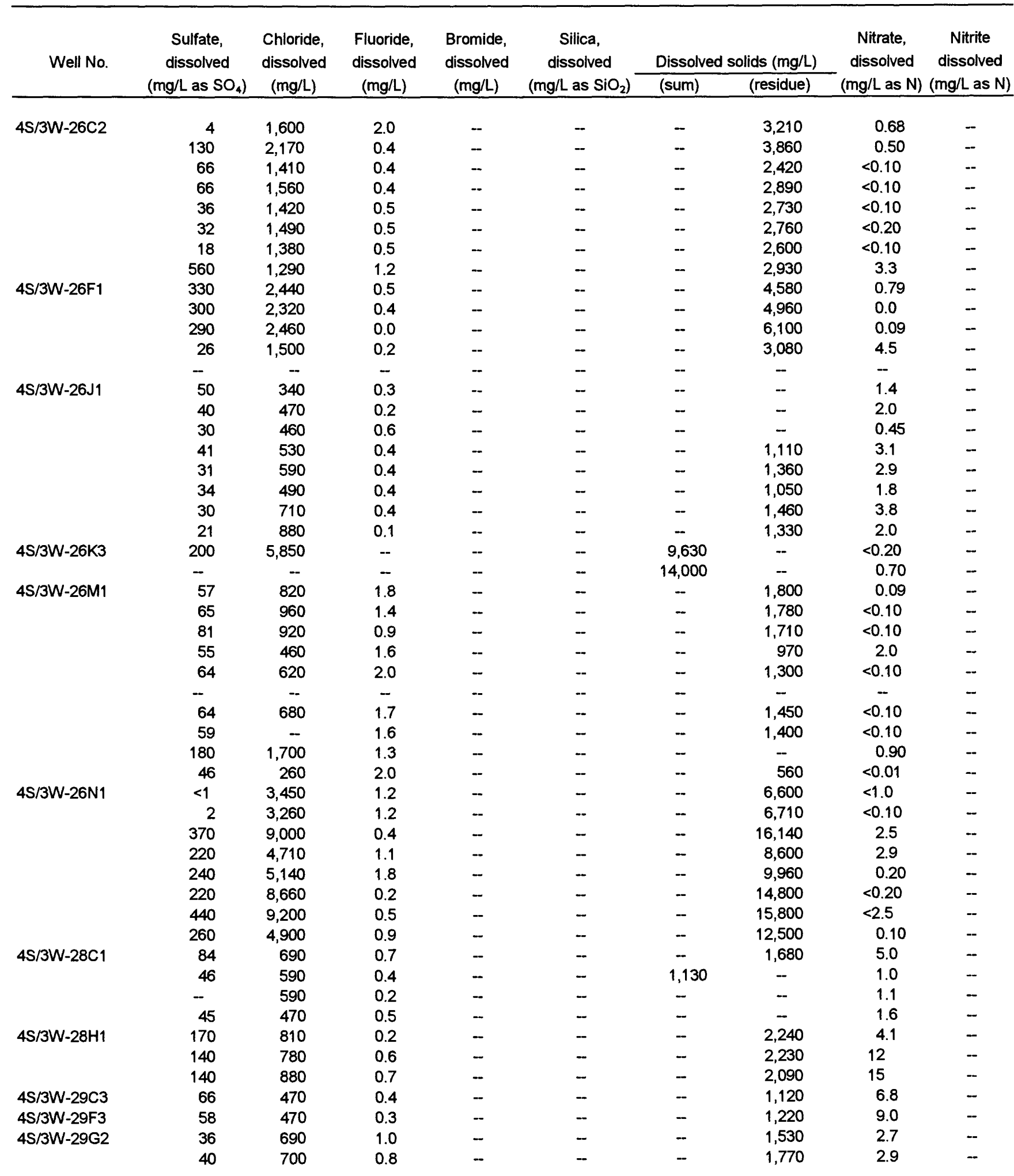


Table 2. Historical water-quality data for selected wells in the Winchester, Menifee, and south Perris ground-water subbasins, California-Continued

\begin{tabular}{|c|c|c|c|c|c|c|c|c|c|}
\hline Well No. & $\begin{array}{c}\mathrm{NO}_{3}+\mathrm{NO}_{2} \\
\text { dissolved } \\
(\mathrm{mg} / \mathrm{L} \text { as } \mathrm{N})\end{array}$ & $\begin{array}{c}\text { Nitrogen } \\
\text { ammonia, } \\
\text { dissolved } \\
\text { (mg/L as } \mathrm{N} \text { ) }\end{array}$ & $\begin{array}{c}\text { Phosphorus, } \\
\text { dissolved } \\
(\mathrm{mg} / \mathrm{L})\end{array}$ & $\begin{array}{c}\text { Phosphorus } \\
\text { ortho, } \\
\text { dissolved } \\
\text { (mg/L as P) }\end{array}$ & $\begin{array}{c}\text { Arsenic, } \\
\text { dissolved } \\
(\mu \mathrm{g} / \mathrm{L})\end{array}$ & $\begin{array}{c}\text { Banium, } \\
\text { dissolved } \\
(\mu g / L)\end{array}$ & $\begin{array}{c}\text { Beryllium, } \\
\text { dissolved } \\
(\mu \mathrm{g} / \mathrm{L})\end{array}$ & $\begin{array}{c}\text { Boron, } \\
\text { dissolved } \\
(\mu \mathrm{g} / \mathrm{L})\end{array}$ & $\begin{array}{c}\text { Cadmium, } \\
\text { dissolved } \\
(\mu g / L)\end{array}$ \\
\hline \multirow[t]{8}{*}{$4 S / 3 W-26 C 2$} & - & - & - & -- & $<10$ & - & $<10$ & 200 & $<10$ \\
\hline & - & - & - & - & - & -- & - & 1,100 & 7.0 \\
\hline & -- & - & - & -- & $<10$ & -- & $<100$ & 700 & $<5$ \\
\hline & - & - & - & - & - & -- & - & 700 & $<5$ \\
\hline & -- & - & - & - & - & -- & - & 700 & $<5$ \\
\hline & -- & 0.40 & - & - & $<10$ & - & $<10$ & 700 & $<1$ \\
\hline & - & -- & - & -- & - & - & - & 600 & $<5$ \\
\hline & -- & $<0.078$ & -- & -- & $<10$ & -- & $<10$ & 3,100 & $<1$ \\
\hline \multirow[t]{5}{*}{$4 S / 3 W-26 F 1$} & -- & - & - & - & - & - & - & 1,500 & - \\
\hline & - & - & - & - & -- & - & -- & 1,600 & -- \\
\hline & - & - & - & -- & -- & - & - & 1,300 & -- \\
\hline & -- & - & - & - & $<10$ & - & $<10$ & 200 & $<10$ \\
\hline & - & - & -- & - & $<10$ & -- & $<10$ & -- & 10 \\
\hline \multirow[t]{8}{*}{ 4S/3W-26J1 } & -- & - & - & - & -- & - & - & 300 & - \\
\hline & - & - & - & -- & - & - & - & 500 & - \\
\hline & - & -- & -- & - & -- & -- & - & 600 & - \\
\hline & -- & -- & - & - & - & -- & - & 410 & - \\
\hline & - & -- & - & -- & - & - & - & 440 & - \\
\hline & - & - & - & -- & - & - & - & 320 & - \\
\hline & - & - & - & -- & - & - & - & 460 & - \\
\hline & - & - & - & -- & -- & - & -- & 200 & - \\
\hline \multirow[t]{2}{*}{$4 S / 3 W-26 K 3$} & - & - & - & - & $<10$ & - & - & - & - \\
\hline & - & - & - & - & -- & - & - & - & - \\
\hline \multirow[t]{10}{*}{$4 \mathrm{~S} / 3 \mathrm{~W}-26 \mathrm{M} 1$} & - & 0.39 & - & - & $<10$ & - & $<10$ & 1,000 & $<10$ \\
\hline & - & 0.20 & - & -- & -- & - & - & 1,200 & - \\
\hline & - & - & - & - & $<10$ & - & $<10$ & 1,300 & 9.0 \\
\hline & - & - & - & -- & $<10$ & - & $<10$ & 1,400 & $<5$ \\
\hline & - & - & - & - & -- & - & - & 1,400 & $<5$ \\
\hline & -- & - & - & - & $<10$ & -- & $<10$ & - & $<1$ \\
\hline & - & - & - & - & - & - & -- & 1,700 & $<5$ \\
\hline & - & 0.31 & -- & - & $<10$ & $<100$ & - & 1,200 & $<1$ \\
\hline & - & - & - & $\cdots$ & - & - & - & - & - \\
\hline & -- & $<0.10$ & -- & - & $<10$ & - & $<10$ & 1,300 & $<1$ \\
\hline \multirow[t]{8}{*}{$4 S / 3 W-26 N 1$} & - & 0.39 & - & - & $<10$ & - & $<10$ & 600 & 10 \\
\hline & - & 13 & -- & -- & - & - & - & 700 & - \\
\hline & - & -- & - & - & $<10$ & - & 10 & 700 & $<10$ \\
\hline & - & - & - & - & 10 & - & $<10$ & 1,300 & $<5$ \\
\hline & - & - & - & - & $<10$ & - & $<10$ & 1,700 & $<5$ \\
\hline & - & 0.16 & - & - & $<10$ & - & $<10$ & 800 & $<1$ \\
\hline & -- & - & - & - & $<10$ & - & $<10$ & 700 & $<10$ \\
\hline & - & - & - & -- & $<10$ & - & $<10$ & 1,000 & $<10$ \\
\hline \multirow[t]{4}{*}{$4 S / 3 W-28 C 1$} & - & - & - & - & -- & -- & - & 280 & - \\
\hline & - & -- & - & - & - & - & - & 450 & - \\
\hline & - & - & - & - & - & - & - & 200 & - \\
\hline & -- & - & - & - & -- & - & - & 500 & - \\
\hline \multirow[t]{3}{*}{$4 \mathrm{~S} / 3 \mathrm{~W}-28 \mathrm{H} 1$} & - & - & - & -- & - & - & - & 370 & - \\
\hline & - & -- & - & -- & - & - & - & 260 & - \\
\hline & - & - & - & -- & -- & - & - & 280 & -- \\
\hline $4 S / 3 W-29 C 3$ & - & - & - & - & - & - & - & 400 & - \\
\hline $4 S / 3 W-29 F 3$ & - & - & -- & - & - & - & - & 400 & - \\
\hline \multirow[t]{2}{*}{$4 S / 3 W-29 G 2$} & - & -- & - & - & - & - & - & 670 & - \\
\hline & - & -- & -- & -- & - & - & - & 580 & - \\
\hline
\end{tabular}


Table 2. Historical water-quality data for selected wells in the Winchester, Menifee, and south Perris ground-water subbasins, California-Continued

\begin{tabular}{|c|c|c|c|c|c|c|c|c|c|}
\hline Well No. & $\begin{array}{l}\text { Chromium, } \\
\text { dissolved } \\
(\mu \mathrm{g} / \mathrm{L}) \\
\end{array}$ & $\begin{array}{c}\text { Cobalt, } \\
\text { dissolved } \\
(\mu g / L)\end{array}$ & $\begin{array}{c}\text { Copper, } \\
\text { dissolved } \\
(\mu g / L) \\
\end{array}$ & $\begin{array}{c}\text { Iron, } \\
\text { dissolved } \\
(\mu \mathrm{g} / \mathrm{L})\end{array}$ & $\begin{array}{c}\text { Lead, } \\
\text { dissolved } \\
(\mu \mathrm{g} / \mathrm{L}) \\
\end{array}$ & $\begin{array}{c}\text { Lithium, } \\
\text { dissolved } \\
(\mu \mathrm{g} / \mathrm{L})\end{array}$ & $\begin{array}{c}\text { Manganese, } \\
\text { dissolved } \\
(\mu \mathrm{g} / \mathrm{L}) \\
\end{array}$ & $\begin{array}{c}\text { Molybdenum, } \\
\text { dissolved } \\
(\mu \mathrm{g} / \mathrm{L}) \\
\end{array}$ & $\begin{array}{c}\text { Nickel, } \\
\text { dissolved } \\
(\mu \mathrm{g} / \mathrm{L}) \\
\end{array}$ \\
\hline \multirow{8}{*}{$4 S / 3 W-26 C 2$} & $<20$ & -- & 10 & -- & $<50$ & -- & - & -- & 10 \\
\hline & -- & - & $<50$ & - & 60 & - & - & - & $<100$ \\
\hline & -- & - & $<10$ & -- & 42 & - & -- & -- & 22 \\
\hline & -- & - & $<10$ & -- & 46 & - & - & - & 35 \\
\hline & -- & $<50$ & - & - & 30 & - & - & -- & 15 \\
\hline & $<10$ & 10 & -- & -- & $<5$ & - & - & -- & $<10$ \\
\hline & - & $<50$ & -- & -- & $<20$ & - & -- & - & $<5$ \\
\hline & $<10$ & - & $<10$ & - & $<5$ & -- & -- & - & $<20$ \\
\hline \multirow[t]{5}{*}{$4 S / 3 W-26 F 1$} & -- & - & - & - & - & - & -- & - & - \\
\hline & - & - & -- & -- & - & - & - & - & - \\
\hline & - & -- & -- & -- & -- & -- & - & - & - \\
\hline & $<20$ & - & 10 & - & $<50$ & - & - & - & 10 \\
\hline & $<20$ & - & - & -- & - & -- & - & - & - \\
\hline \multirow[t]{8}{*}{$4 S / 3 W-26 J 1$} & -- & - & - & - & - & - & - & - & -- \\
\hline & - & - & - & -- & -- & - & - & - & - \\
\hline & - & - & -- & -- & - & - & - & -- & -- \\
\hline & -- & - & - & - & - & - & -- & - & - \\
\hline & - & - & -- & - & - & -- & - & - & - \\
\hline & - & - & -- & -- & - & -- & - & -- & - \\
\hline & - & - & -- & - & - & - & - & -- & - \\
\hline & -- & -- & -- & - & - & -- & -- & - & - \\
\hline \multirow[t]{2}{*}{$4 S / 3 W-26 K 3$} & - & - & -- & - & $<5$ & -- & - & - & - \\
\hline & - & -- & - & - & -- & - & -- & - & - \\
\hline \multirow[t]{10}{*}{$4 S / 3 W-26 M 1$} & $<20$ & - & 10 & - & $<50$ & - & - & - & $<10$ \\
\hline & - & - & - & - & - & - & - & - & - \\
\hline & $<20$ & - & $<10$ & - & 30 & - & - & - & $<10$ \\
\hline & - & - & $<10$ & - & $<20$ & - & - & - & $<5$ \\
\hline & - & $<50$ & - & - & $<20$ & -- & - & - & 10 \\
\hline & $<10$ & $<10$ & - & - & $<5$ & - & - & - & $<10$ \\
\hline & - & $<50$ & - & - & $<20$ & - & -- & -- & $<5$ \\
\hline & $<10$ & -- & $<10$ & - & $<5$ & - & - & - & 20 \\
\hline & -- & -- & - & - & - & - & - & -- & - \\
\hline & $<10$ & -- & $<10$ & - & 5.0 & - & - & -- & $<20$ \\
\hline \multirow[t]{8}{*}{$4 S / 3 W-26 N 1$} & $<20$ & -- & 20 & - & $<50$ & - & - & - & $<10$ \\
\hline & -- & -- & - & - & - & - & - & - & - \\
\hline & $<10$ & - & 60 & - & 40 & -- & - & - & $<10$ \\
\hline & $<20$ & -- & 28 & - & 200 & -- & - & -- & 120 \\
\hline & -- & 110 & - & -- & 290 & - & - & - & 110 \\
\hline & $<10$ & $<10$ & -- & - & $<5$ & - & - & -- & $<10$ \\
\hline & $<10$ & $<10$ & $<10$ & - & $<10$ & -- & - & -- & $<20$ \\
\hline & $<10$ & -- & 30 & - & $<5$ & - & - & - & $<20$ \\
\hline \multirow[t]{4}{*}{$4 S / 3 W-28 C 1$} & -- & - & -- & -- & - & - & -- & - & - \\
\hline & - & -- & -- & -- & -- & -- & -- & -- & -- \\
\hline & -- & -- & -- & - & -- & -- & - & - & -- \\
\hline & - & -- & - & - & - & -- & - & - & - \\
\hline \multirow[t]{3}{*}{$4 \mathrm{~S} / 3 \mathrm{~W}-28 \mathrm{H} 1$} & -- & -- & -- & - & - & - & - & - & - \\
\hline & - & - & - & - & -- & - & - & -- & - \\
\hline & - & - & -- & - & -- & - & - & - & - \\
\hline $4 S / 3 W-29 C 3$ & - & -- & - & -- & -- & - & - & - & - \\
\hline $4 S / 3 W-29 F 3$ & - & - & - & - & - & - & - & - & - \\
\hline \multirow[t]{2}{*}{$4 S / 3 W-29 G 2$} & -- & -- & -- & - & - & - & - & - & - \\
\hline & - & - & -- & -- & -- & - & -- & - & -- \\
\hline
\end{tabular}


Table 2. Historical water-quality data for selected wells in the Winchester, Menifee, and south Perris ground-water subbasins, California-Continued

\begin{tabular}{|c|c|c|c|c|c|c|}
\hline Well No. & $\begin{array}{c}\text { Selenium, } \\
\text { dissolved } \\
(\mu \mathrm{g} / \mathrm{L})\end{array}$ & $\begin{array}{c}\text { Silver, } \\
\text { dissolved } \\
(\mu g / L)\end{array}$ & $\begin{array}{c}\text { Strontium, } \\
\text { dissolved } \\
(\mu g / L)\end{array}$ & $\begin{array}{c}\text { Vanadium, } \\
\text { dissolved } \\
(\mu \mathrm{g} / \mathrm{L})\end{array}$ & $\begin{array}{c}\text { Zinc, } \\
\text { dissolved } \\
(\mu g / L)\end{array}$ & Source of data \\
\hline \multirow[t]{8}{*}{$4 S / 3 W-26 C 2$} & $<5$ & 10 & - & - & 20 & Babcock \\
\hline & - & $<25$ & -- & - & 560 & EMWD \\
\hline & $<5$ & $<25$ & - & - & 450 & EMWD/Babcock \\
\hline & -- & $<25$ & -- & - & 220 & EMWD \\
\hline & - & $<25$ & - & - & $<50$ & EMWD \\
\hline & $<5$ & $<10$ & - & -- & 360 & EMWD/Babcock \\
\hline & -- & $<25$ & - & - & 1,900 & EMWD \\
\hline & 23 & $<10$ & - & - & 480 & Babcock \\
\hline \multirow[t]{5}{*}{$4 S / 3 W-26 F 1$} & - & - & - & - & -- & RCFCD \\
\hline & - & -- & -- & -- & -- & RCFCD \\
\hline & - & -- & - & - & -- & RCFCD \\
\hline & $<5$ & 10 & - & - & 20 & Babcock \\
\hline & $<5$ & - & -- & - & - & Babcock \\
\hline \multirow[t]{8}{*}{ 4S/3W-26J1 } & -- & - & - & -- & - & RCFCD \\
\hline & - & - & - & - & - & RCFCD \\
\hline & -- & -- & - & - & - & RCFCD \\
\hline & - & - & -- & -- & - & RCFCD \\
\hline & - & -- & -- & -- & - & RCFCD \\
\hline & - & - & - & - & - & RCFCD \\
\hline & - & - & -- & - & -- & RCFCD \\
\hline & - & -. & -- & - & -- & RCFCD \\
\hline \multirow[t]{2}{*}{$4 S / 3 W-26 K 3$} & - & -- & -- & - & - & Babcock \\
\hline & - & -- & -- & - & -- & Babcock \\
\hline \multirow[t]{10}{*}{$4 S / 3 W-26 M 1$} & $<5$ & 10 & - & - & 20 & Babcock \\
\hline & - & -- & - & - & - & Babcock \\
\hline & $<5$ & $<25$ & - & - & 400 & EMWD/Babcock \\
\hline & $<5$ & $<25$ & - & - & 650 & EMWD/Babcock \\
\hline & - & $<25$ & - & -- & 90 & EMWD \\
\hline & $<5$ & $<10$ & - & -- & 540 & Babcock \\
\hline & - & $<25$ & - & -- & 80 & EMWD \\
\hline & $<5$ & $<10$ & - & -- & 40 & Babcock \\
\hline & -- & - & - & - & - & Babcock \\
\hline & $<5$ & $<10$ & - & - & 130 & Babcock \\
\hline \multirow[t]{8}{*}{$4 S / 3 W-26 N 1$} & $<5$ & 10 & - & - & 50 & Babcock \\
\hline & - & -- & - & - & - & Babcock \\
\hline & 10 & $<10$ & - & - & 50 & EMWD/Babcock \\
\hline & $<10$ & $<25$ & - & - & 860 & EMWD/Babcock \\
\hline & $<5$ & $<25$ & - & -- & 920 & EMWD/Babcock \\
\hline & $<5$ & $<10$ & - & - & 1,100 & EMWD/Babcock \\
\hline & $<5$ & $<10$ & -- & - & 80 & Babcock \\
\hline & $<25$ & $<10$ & - & - & 180 & Babcock \\
\hline \multirow[t]{4}{*}{$4 S / 3 W-28 C 1$} & - & - & - & - & -- & RCFCD \\
\hline & - & - & - & - & -- & RCFCD \\
\hline & - & - & - & - & - & RCFCD \\
\hline & - & -- & -- & -- & - & RCFCD \\
\hline \multirow[t]{3}{*}{$4 \mathrm{~S} / 3 \mathrm{~W}-28 \mathrm{H} 1$} & -- & - & -- & - & - & RCFCD \\
\hline & - & -- & - & - & -- & RCFCD \\
\hline & - & - & -- & -- & - & RCFCD \\
\hline $4 S / 3 W-29 C 3$ & - & -- & -- & - & -- & EMWD \\
\hline $4 S / 3 W-29 F 3$ & - & - & - & - & -- & EMWD \\
\hline \multirow[t]{2}{*}{$4 S / 3 W-29 G 2$} & - & - & -- & -- & - & RCFCD \\
\hline & - & - & - & - & - & RCFCD \\
\hline
\end{tabular}


Table 2. Historical water-quality data for selected wells in the Winchester, Menifee, and south Perris ground-water subbasins, California-Continued

\begin{tabular}{|c|c|c|c|c|c|c|c|c|}
\hline \multirow[t]{2}{*}{ Well No. } & \multirow[t]{2}{*}{ Date } & \multirow{2}{*}{$\begin{array}{l}\text { Water level } \\
\text { (ft blw LSD) }\end{array}$} & \multirow{2}{*}{$\begin{array}{l}\text { Well depth } \\
\text { (ft) }\end{array}$} & \multirow{2}{*}{$\begin{array}{c}\text { Altitude } \\
\text { of LSD (ft) }\end{array}$} & \multicolumn{2}{|c|}{ Specific conductance $(\mu \mathrm{S} / \mathrm{cm})$} & \multirow{2}{*}{$\begin{array}{c}\text { pH (lab) } \\
\text { (Std. units) }\end{array}$} & \multirow{2}{*}{$\begin{array}{c}\text { Water } \\
\text { temperature } \\
\left({ }^{\circ} \mathrm{C}\right) \\
\end{array}$} \\
\hline & & & & & Field & Lab & & \\
\hline & $6 / 14 / 73$ & -- & & & -- & 2,300 & 8.0 & 21.0 \\
\hline & $5 / 8 / 74$ & -- & & & -- & 3,050 & 7.5 & 21.5 \\
\hline & $5 / 13 / 76$ & -- & & & - & 2,600 & 7.8 & 22.0 \\
\hline & $4 / 20 / 77$ & -- & & & -- & - & - & 22.0 \\
\hline \multirow[t]{2}{*}{$4 S / 3 W-29 K 1$} & $3 / 27 / 63$ & .- & - & -- & -- & 2,400 & 7.4 & - \\
\hline & $4 / 19 / 77$ & .- & & & -- & 2,910 & 7.7 & - \\
\hline \multirow{7}{*}{ 4S/3W-29Q1 } & $3 / 31 / 59$ & -- & 624 & 1,417 & -- & 2,380 & 7.6 & 21.0 \\
\hline & $4 / 10 / 60$ & -- & & & -- & - & 7.2 & - \\
\hline & $4 / 12 / 61$ & -- & & & - & 2,400 & 8.0 & -- \\
\hline & 9/27/61 & -- & & & - & 2,450 & 8.0 & 23.0 \\
\hline & $5 / 4 / 62$ & -- & & & -- & 2,250 & 7.3 & 24.5 \\
\hline & $10 / 3 / 69$ & -- & & & _- & 1,860 & 7.0 & 20.0 \\
\hline & $7 / 14 / 70$ & -- & & & -- & -- & 7.4 & - \\
\hline $4 S / 3 W-32 B 1$ & $3 / 30 / 65$ & -- & -- & -- & .- & 6,200 & 7.3 & -- \\
\hline \multirow[t]{27}{*}{$5 S / 2 W-19 N 1$} & $12 / 18 / 53$ & - & 290.5 & 1,459 & -- & 888 & 7.2 & -- \\
\hline & $12 / 16 / 54$ & -- & & & -- & 867 & 7.2 & -- \\
\hline & $8 / 10 / 55$ & _- & & & -- & 997 & 7.1 & 22.0 \\
\hline & $9 / 18 / 56$ & _- & & & -- & 1,060 & -- & 22.0 \\
\hline & $9 / 18 / 57$ & -- & & & -- & 781 & 8.0 & 22.0 \\
\hline & $10 / 14 / 58$ & -- & & & _- & 738 & 7.7 & 29.0 \\
\hline & $9 / 16 / 59$ & -- & & & -- & 635 & 7.0 & 22.0 \\
\hline & $9 / 14 / 60$ & -- & & & -- & 657 & 8.2 & 23.5 \\
\hline & $10 / 5 / 61$ & -- & & & -- & 658 & 7.9 & - \\
\hline & $9 / 25 / 62$ & -- & & & -- & 668 & 7.0 & 22.0 \\
\hline & $3 / 14 / 63$ & -- & & & -- & 660 & 6.7 & -- \\
\hline & 9/27/63 & -- & & & -- & 698 & 7.0 & 23.5 \\
\hline & $8 / 13 / 64$ & -- & & & -- & 710 & 7.3 & 22.0 \\
\hline & $9 / 27 / 65$ & - & & & -- & 755 & 7.0 & 24.5 \\
\hline & $9 / 15 / 66$ & -- & & & - & 789 & 7.5 & 25.5 \\
\hline & 9/29/67 & -- & & & -- & 848 & 7.9 & 23.5 \\
\hline & $10 / 15 / 68$ & -- & & & -- & 1,040 & 7.7 & 26.5 \\
\hline & $10 / 23 / 69$ & - & & & -- & 990 & 7.6 & 26.5 \\
\hline & $11 / 18 / 70$ & -- & & & -- & 851 & 7.7 & 24.5 \\
\hline & $11 / 4 / 71$ & -- & & & -- & 649 & 8.0 & 24.5 \\
\hline & $5 / 11 / 72$ & -- & & & -- & 637 & 8.2 & 23.0 \\
\hline & $9 / 27 / 73$ & -- & & & -- & 700 & 8.0 & 22.0 \\
\hline & $9 / 20 / 74$ & -- & & & -- & 883 & 8.5 & 27.0 \\
\hline & $9 / 30 / 75$ & -- & & & -- & 888 & 8.2 & 21.5 \\
\hline & $9 / 20 / 76$ & -- & & & -- & 949 & 7.3 & 22.0 \\
\hline & $10 / 28 / 77$ & -- & & & -- & 1,050 & 8.1 & 22.0 \\
\hline & $5 / 23 / 79$ & -. & & & -. & 989 & 7.8 & 17.0 \\
\hline $5 \mathrm{~S} / 2 \mathrm{~W}-21 \mathrm{M} 1$ & $6 / 9 / 93$ & & 260 & 1,480 & 2,150 & 2,120 & 7.2 & 24.0 \\
\hline \multirow[t]{2}{*}{$5 \mathrm{~S} / 2 \mathrm{~W}-21 \mathrm{M} 2$} & $6 / 9 / 93$ & 26.21 & 260 & 1,490 & 3,980 & 3,980 & 6.5 & 23.0 \\
\hline & $1 / 21 / 94$ & -- & & & 3,690 & 3,720 & 6.7 & 22.0 \\
\hline \multirow[t]{2}{*}{$5 S / 2 W-27 N 1$} & 6/8/93 & -- & 59 & 1,491 & 6,780 & 6,720 & 6.3 & 21.5 \\
\hline & $1 / 20 / 94$ & _- & & & 6,630 & 6,690 & 6.7 & 21.0 \\
\hline $5 \mathrm{~S} / 2 \mathrm{~W}-28 \mathrm{E} 1$ & $7 / 13 / 94$ & -- & 400.3 & 1,459 & 14,800 & 14,600 & 6.7 & 22.5 \\
\hline \multirow{3}{*}{$5 \mathrm{~S} / 2 \mathrm{~W}-29 \mathrm{~N} 1$} & $6 / 7 / 93$ & 6.4 & 113.5 & 1,450 & 6,070 & 6,120 & 6.6 & 20.0 \\
\hline & $1 / 20 / 94$ & 8.48 & & & 5,050 & 5,100 & 6.1 & 21.5 \\
\hline & $6 / 24 / 94$ & -- & & & 5,200 & 5,180 & 6 & 22.0 \\
\hline $5 \mathrm{~S} / 2 \mathrm{~W}-30 \mathrm{C} 1$ & $6 / 83$ & -- & 355.7 & 1,452 & -- & -- & - & -- \\
\hline \multirow[t]{4}{*}{$5 \mathrm{~S} / 2 \mathrm{~W}-30 \mathrm{D} 2$} & $11 / 91$ & - & 355 & 1,455 & -- & 4,210 & 7.9 & -. \\
\hline & $6 / 25 / 93$ & -- & & & 4,330 & 4,400 & 6.2 & 22.5 \\
\hline & $1 / 20 / 94$ & -- & & & 4,400 & 4,460 & 6.7 & 22.5 \\
\hline & $6 / 17 / 94$ & - & & & 4,450 & 4,480 & 6.2 & 22.5 \\
\hline $5 \mathrm{~S} / 2 \mathrm{~W}-30 \mathrm{~J} 1$ & $4 / 6 / 75$ & - & 300 & 1,445 & - & 7,800 & 6.8 & - \\
\hline $5 \mathrm{~S} / 2 \mathrm{~W}-31 \mathrm{~A} 1$ & $11 / 6 / 92$ & -- & - & - & -- & 2,000 & 7.6 & -- \\
\hline $5 \mathrm{~S} / 2 \mathrm{~W}-31 \mathrm{~N} 1$ & $4 / 24 / 75$ & -- & 33 & 1,450 & - & 893 & 7.8 & -- \\
\hline
\end{tabular}


Table 2. Historical water-quality data for selected wells in the Winchester, Menifee, and south Perris ground-water subbasins, California-Continued

\begin{tabular}{|c|c|c|c|c|c|c|c|c|}
\hline \multirow[t]{2}{*}{ Well No. } & \multirow{2}{*}{$\begin{array}{c}\text { Hardness } \\
\left(\mathrm{mg} / \mathrm{L} \text { as } \mathrm{CaCO}_{3}\right)\end{array}$} & \multirow{2}{*}{$\begin{array}{c}\text { Calcium, } \\
\text { dissolved } \\
(\mathrm{mg} / \mathrm{L})\end{array}$} & \multirow{2}{*}{$\begin{array}{c}\text { Magnesium, } \\
\text { dissolved } \\
\text { (mg/L) }\end{array}$} & \multirow{2}{*}{$\begin{array}{l}\text { Sodium, } \\
\text { dissolved } \\
(\mathrm{mg} / \mathrm{L})\end{array}$} & \multirow{2}{*}{$\begin{array}{l}\text { Potassium, } \\
\text { dissolved } \\
\text { (mg/L as } \mathrm{K} \text { ) }\end{array}$} & \multirow{2}{*}{$\begin{array}{c}\text { Bicarbonate } \\
\left(\mathrm{mg} / \mathrm{L} \text { as } \mathrm{HCO}_{3}\right)\end{array}$} & \multicolumn{2}{|c|}{ Alkalinity (mg/L as $\mathrm{CaCO}_{3}$ ) } \\
\hline & & & & & & & Field & Lab \\
\hline & 570 & 170 & 37.0 & 220.0 & 3 & 84 & -- & 68 \\
\hline & 70 & 240 & 26.0 & 270.0 & 4 & 87 & - & 71 \\
\hline & 690 & 210 & 38.0 & 230.0 & 6 & 93 & - & 76 \\
\hline & - & -- & -- & -- & - & - & - & -- \\
\hline \multirow[t]{2}{*}{$4 S / 3 W-29 K 1$} & 620 & 200 & 32.0 & 280.0 & 4 & 160 & - & - \\
\hline & 900 & 290 & 43.0 & 240.0 & 10 & 150 & - & 130 \\
\hline \multirow[t]{7}{*}{$4 S / 3 W-29 Q 1$} & 790 & 220 & 60.0 & 170.0 & -- & 92 & - & -- \\
\hline & 690 & 220 & 37.0 & 170.0 & 5 & 100 & - & - \\
\hline & 730 & 260 & 19.0 & 160.0 & 7 & 98 & - & - \\
\hline & 800 & 280 & 24.0 & 190.0 & 11 & 100 & - & -- \\
\hline & 740 & 240 & 31.0 & 170.0 & 6 & 92 & - & -- \\
\hline & 700 & 220 & 38.0 & 97.0 & 5 & 150 & - & -- \\
\hline & 760 & 240 & 37.0 & 118.0 & 5 & 140 & - & -- \\
\hline $4 S / 3 W-32 B 1$ & 2,000 & 640 & 86.0 & 500.0 & 10 & 85 & - & - \\
\hline \multirow[t]{27}{*}{$5 S / 2 W-19 N 1$} & - & 77 & 15.0 & 76.0 & 4 & 180 & - & - \\
\hline & - & 73 & 15.6 & 71.8 & 3 & 160 & - & - \\
\hline & 290 & 82 & 20.0 & 84.0 & 4 & 130 & - & - \\
\hline & 320 & 86 & 26.0 & 95.0 & -- & 150 & - & -- \\
\hline & 230 & 60 & 19.0 & 72.0 & - & 130 & - & - \\
\hline & 180 & 52 & 13.0 & 64.0 & -- & 140 & - & - \\
\hline & 160 & 47 & 10.0 & 58.0 & -- & 120 & - & - \\
\hline & 190 & 54 & 12.0 & 64.0 & 4 & 120 & - & - \\
\hline & 140 & 50 & 4.0 & 55.0 & 6 & 120 & - & - \\
\hline & 170 & 57 & 7.0 & 62.0 & 3 & 120 & -- & -- \\
\hline & 170 & 50 & 10.0 & 70.0 & 2 & 120 & - & - \\
\hline & 160 & 48 & 10.0 & 58.0 & 2 & 110 & - & -- \\
\hline & 210 & 58 & 15.0 & 67.0 & 5 & 110 & - & 90 \\
\hline & 200 & 57 & 13.0 & 69.0 & 3 & 130 & - & 110 \\
\hline & 210 & 59 & 16.0 & 70.0 & 3 & 130 & - & 110 \\
\hline & 220 & 61 & 17.0 & 81.0 & 3 & 150 & - & 120 \\
\hline & 260 & 71 & 20.0 & 99.0 & 2 & 170 & - & 140 \\
\hline & 250 & 69 & 19.0 & 98.0 & 2 & 190 & - & 150 \\
\hline & 240 & 61 & 21.0 & 76.0 & 6 & 160 & - & 130 \\
\hline & 150 & 42 & 11.0 & 61.0 & 3 & 110 & - & 86 \\
\hline & 150 & 39 & 12.0 & 58.0 & 3 & 110 & - & 87 \\
\hline & 190 & 46 & 14.0 & 77.0 & 2 & 120 & - & 99 \\
\hline & 220 & 85 & 1.1 & 88.0 & 9 & 150 & - & 130 \\
\hline & 230 & 67 & 15.0 & 83.0 & 3 & 140 & - & 120 \\
\hline & 240 & 67 & 17.0 & 99.0 & 3 & 150 & - & 120 \\
\hline & 300 & 80 & 24.0 & 120.0 & 3 & 150 & - & 120 \\
\hline & 270 & 75 & 22.0 & 110.0 & 2 & -- & - & 120 \\
\hline $5 S / 2 W-21 M 1$ & 860 & 190 & 93.0 & 120.0 & - & - & 640 & - \\
\hline \multirow[t]{2}{*}{$5 S / 2 W-21 M 2$} & 1,700 & 380 & 190.0 & 140.0 & - & - & 400 & - \\
\hline & 1,700 & 370 & 180.0 & 140.0 & 18 & - & 360 & - \\
\hline \multirow[t]{2}{*}{$5 \mathrm{~S} / 2 \mathrm{~W}-27 \mathrm{~N} 1$} & 1,500 & 370 & 140.0 & 950.0 & - & - & 340 & - \\
\hline & 1,600 & 410 & 150.0 & 940.0 & 9 & - & 350 & -- \\
\hline $5 S / 2 W-28 E 1$ & 1,600 & 490 & 92.0 & 890.0 & 31 & -- & 430 & - \\
\hline \multirow[t]{3}{*}{$5 S / 2 W-29 N 1$} & 1,700 & 380 & 180.0 & 1300.0 & - & -- & 210 & - \\
\hline & 1,700 & 430 & 140.0 & 440.0 & 10 & - & 160 & - \\
\hline & 1,700 & 440 & 150.0 & 480.0 & 10 & -- & 160 & - \\
\hline $5 S / 2 W-30 C 1$ & -- & 96 & 35.4 & 140.0 & 4 & 150 & - & - \\
\hline \multirow[t]{4}{*}{$5 S / 2 W-30 D 2$} & 1,200 & 390 & 61.0 & 270.0 & 8 & 200 & - & 160 \\
\hline & 1,500 & 410 & 120.0 & 290.0 & - & -- & 170 & - \\
\hline & 1,600 & 430 & 120.0 & 280.0 & 7 & - & 160 & - \\
\hline & 1,600 & 410 & 130.0 & 290.0 & 9 & - & 170 & - \\
\hline $5 \mathrm{~S} / 2 \mathrm{~W}-30 \mathrm{~J} 1$ & 1,400 & 330 & 135.0 & 830.0 & 8 & 230 & - & -- \\
\hline $5 S / 2 W-31 A 1$ & -- & 150 & 70.0 & 250.0 & 32 & 340 & - & - \\
\hline $5 S / 2 W-31 N 1$ & 250 & 63 & 22.0 & 79.0 & 6 & 130 & - & 100 \\
\hline
\end{tabular}


Table 2. Historical water-quality data for selected wells in the Winchester, Menifee, and south Perris ground-water subbasins, California-Continued

\begin{tabular}{|c|c|c|c|c|c|c|c|c|c|}
\hline \multirow[t]{2}{*}{ Well No. } & \multirow{2}{*}{$\begin{array}{c}\text { Sulfate, } \\
\text { dissolved } \\
\left(\mathrm{mg} / \mathrm{L} \text { as } \mathrm{SO}_{4}\right)\end{array}$} & \multirow{2}{*}{$\begin{array}{c}\text { Chloride, } \\
\text { dissolved } \\
(\mathrm{mg} / \mathrm{L})\end{array}$} & \multirow{2}{*}{$\begin{array}{c}\text { Fluoride, } \\
\text { dissolved } \\
(\mathrm{mg} / \mathrm{L})\end{array}$} & \multirow{2}{*}{$\begin{array}{c}\text { Bromide, } \\
\text { dissolved } \\
(\mathrm{mg} / \mathrm{L})\end{array}$} & \multirow{2}{*}{$\begin{array}{c}\text { Silica, } \\
\text { dissolved } \\
\left(\mathrm{mg} / \mathrm{L} \text { as } \mathrm{SiO}_{2}\right) \\
\end{array}$} & \multicolumn{2}{|c|}{ Dissolved solids (mg/L) } & \multirow{2}{*}{$\begin{array}{c}\text { Nitrate, } \\
\text { dissolved } \\
\text { (mg/L as } \mathrm{N})\end{array}$} & \multirow{2}{*}{$\begin{array}{c}\text { Nitrite } \\
\text { dissolved } \\
\text { (mg/L as N) } \\
\end{array}$} \\
\hline & & & & & & (sum) & (residue) & & \\
\hline & 35 & 650 & 0.7 & -- & - & -- & 2,040 & 3.3 & - \\
\hline & 40 & 830 & 0.7 & -- & - & - & 1,940 & 2.9 & - \\
\hline & 40 & 750 & 0.9 & - & - & -- & 2,230 & 3.2 & - \\
\hline & -- & - & - & -- & - & - & 1,630 & -- & -- \\
\hline \multirow[t]{2}{*}{$4 S / 3 W-29 K 1$} & 25 & 720 & 0.4 & - & 16 & - & 1,560 & 1.1 & - \\
\hline & 65 & 870 & - & -- & 11 & -- & 2,520 & 6.1 & - \\
\hline \multirow[t]{7}{*}{ 4S/3W-29Q1 } & 45 & 710 & 0.7 & - & -- & - & 1,260 & 1.6 & -- \\
\hline & 38 & 660 & 0.6 & - & - & -. & -- & 3.2 & - \\
\hline & 20 & 720 & 0.4 & - & -- & -- & - & 2.3 & - \\
\hline & 150 & 690 & 0.6 & - & - & - & - & 1.8 & -- \\
\hline & 32 & 710 & 0.4 & -- & -- & -- & - & 1.8 & -- \\
\hline & 47 & 510 & 0.4 & - & - & - & 1,110 & 3.6 & -- \\
\hline & 58 & 580 & 0.3 & -- & - & - & 1,690 & 5.7 & - \\
\hline 4S/3W-32B1 & 35 & 3,000 & 0.4 & - & - & - & 5,340 & 0.45 & -- \\
\hline \multirow[t]{27}{*}{$5 S / 2 W-19 N 1$} & 53 & 150 & 0.3 & - & -- & -- & 600 & 4.3 & - \\
\hline & 56 & 140 & 0.0 & -- & -- & - & 570 & 6.6 & - \\
\hline & 65 & 200 & 0.1 & - & -- & - & 760 & 4.9 & - \\
\hline & 78 & 220 & 0.0 & - & - & -. & -- & 2.0 & - \\
\hline & 55 & 150 & 0.2 & - & - & -- & - & 2.0 & -- \\
\hline & 30 & 110 & 0.0 & - & - & -- & - & 4.5 & -- \\
\hline & 30 & 100 & 0.0 & - & -- & -- & - & 2.0 & -- \\
\hline & 60 & 99 & 0.1 & - & - & -- & - & 4.5 & -- \\
\hline & 35 & 89 & 0.3 & -- & -- & - & -- & 3.6 & - \\
\hline & 36 & 1,220 & 0.1 & - & - & - & 420 & 3.4 & - \\
\hline & 40 & 110 & 0.2 & - & 55 & - & 490 & 5.9 & - \\
\hline & 28 & 120 & 0.2 & -- & - & - & 390 & 2.7 & -- \\
\hline & 40 & 150 & 0.3 & - & - & -- & 460 & 4.1 & - \\
\hline & 36 & 110 & 0.3 & -- & -- & - & 530 & 11 & - \\
\hline & 42 & 120 & 0.3 & -- & -- & - & 470 & 14 & - \\
\hline & 47 & 120 & 0.3 & -- & -- & - & 620 & 17 & - \\
\hline & 84 & 140 & 0.1 & -- & - & - & 600 & 17 & - \\
\hline & 89 & 120 & 0.3 & -- & -- & - & 600 & 9.3 & -- \\
\hline & 70 & 130 & 0.2 & -- & - & - & 500 & 7.2 & -- \\
\hline & 39 & 100 & 0.2 & - & - & - & 440 & 6.8 & -- \\
\hline & 40 & 96 & 0.3 & - & - & -- & 400 & 5.7 & - \\
\hline & 51 & 110 & 0.2 & -- & -- & - & 520 & 7.9 & -- \\
\hline & 72 & 130 & 0.2 & -- & - & - & 590 & 9.0 & - \\
\hline & 76 & 120 & 0.3 & -- & - & - & 600 & 13 & - \\
\hline & 120 & 120 & 0.4 & - & -- & - & 620 & 12 & -- \\
\hline & 180 & 130 & 0.6 & -- & - & - & 850 & 17 & -- \\
\hline & 160 & 130 & 0.4 & -- & - & - & 720 & 15 & -- \\
\hline $5 S / 2 W-21 M 1$ & 140 & 190 & - & -- & 58 & - & - & 35 & $<0.02$ \\
\hline \multirow[t]{2}{*}{$5 S / 2 W-21 M 2$} & 360 & 570 & - & - & 59 & -- & - & 140 & $<0.02$ \\
\hline & 350 & 540 & - & - & 59 & -- & - & 140 & 0.40 \\
\hline \multirow[t]{2}{*}{$5 S / 2 W-27 N 1$} & 1,120 & 1,460 & - & -- & - & -- & -- & 3.3 & $<0.25$ \\
\hline & 1,110 & 1,490 & -- & -- & -- & -- & - & 6.4 & 0.03 \\
\hline $5 \mathrm{~S} / 2 \mathrm{~W}-28 \mathrm{E} 1$ & 930 & 4,800 & $<0.10$ & 9.4 & 57 & 8,020 & -- & 2.2 & $<0.01$ \\
\hline $5 S / 2 W-29 N 1$ & 150 & 110 & -- & - & - & - & - & - & $<0.02$ \\
\hline & 920 & 1,160 & -- & - & 54 & -- & - & 12 & 0.03 \\
\hline & 930 & 1,160 & $<2.5$ & - & 58 & -- & - & 13 & $<0.010$ \\
\hline $5 S / 2 W-30 C 1$ & 51 & 300 & -- & - & 43 & - & - & - & -- \\
\hline $5 S / 2 W-30 D 2$ & 280 & 1,100 & - & - & 69 & - & 3,280 & 12 & - \\
\hline & -- & - & - & -- & 66 & - & -- & - & -- \\
\hline & -- & - & - & - & 63 & -- & - & 12 & \\
\hline & - & - & - & - & 59 & -- & - & 12 & $<0.010$ \\
\hline 5S/2W-30J1 & 710 & 1,630 & 0.3 & - & -- & -- & 4,220 & 6.1 & -- \\
\hline $5 S / 2 W-31 A 1$ & 750 & 100 & 0.7 & - & - & 1,520 & 1,610 & $<0.09$ & - \\
\hline $5 S / 2 W-31 N 1$ & 100 & 120 & 0.3 & - & - & - & 590 & 15 & - \\
\hline
\end{tabular}


Table 2. Historical water-quality data for selected wells in the Winchester, Menifee, and south Perris ground-water subbasins, California--Continued

\begin{tabular}{|c|c|c|c|c|c|c|c|c|c|}
\hline Well No. & $\begin{array}{c}\mathrm{NO}_{3}+\mathrm{NO}_{2} \\
\text { dissolved } \\
(\mathrm{mg} / \mathrm{L} \text { as } \mathrm{N})\end{array}$ & $\begin{array}{c}\text { Nitrogen } \\
\text { ammonia, } \\
\text { dissolved } \\
\text { (mg/L as } \mathrm{N} \text { ) }\end{array}$ & $\begin{array}{l}\text { Phosphorus, } \\
\text { dissolved } \\
\text { (mg/L) }\end{array}$ & $\begin{array}{c}\text { Phosphorus } \\
\text { ortho, } \\
\text { dissolved } \\
\text { (mg/L as P) }\end{array}$ & $\begin{array}{c}\text { Arsenic, } \\
\text { dissolved } \\
(\mu \mathrm{g} / \mathrm{L})\end{array}$ & $\begin{array}{c}\text { Barium, } \\
\text { dissolved } \\
(\mu \mathrm{g} / \mathrm{L})\end{array}$ & $\begin{array}{c}\text { Beryllium, } \\
\text { dissolved } \\
(\mu \mathrm{g} / \mathrm{L})\end{array}$ & $\begin{array}{c}\text { Boron, } \\
\text { dissolved } \\
(\mu \mathrm{g} / \mathrm{L})\end{array}$ & $\begin{array}{c}\text { Cadmium, } \\
\text { dissolved } \\
(\mu \mathrm{g} / \mathrm{L})\end{array}$ \\
\hline & - & - & - & - & - & -- & - & 500 & -- \\
\hline & -- & - & -- & - & - & - & - & 770 & - \\
\hline & - & -- & - & -- & - & - & - & 620 & -- \\
\hline & - & - & - & - & - & - & -- & - & - \\
\hline \multirow[t]{2}{*}{$4 S / 3 W-29 K 1$} & - & -- & -- & -- & -- & - & -- & 690 & - \\
\hline & -- & -- & -- & - & - & -- & - & 200 & - \\
\hline \multirow[t]{7}{*}{$4 S / 3 W-29 Q 1$} & - & -- & - & - & - & - & - & 400 & -- \\
\hline & - & -- & - & -- & - & -. & - & - & - \\
\hline & - & -- & - & - & - & - & - & 200 & -- \\
\hline & - & - & -- & -- & -- & - & -- & 200 & - \\
\hline & - & -- & - & -- & -- & - & -- & 400 & - \\
\hline & - & - & - & - & - & - & - & 230 & - \\
\hline & - & -- & -- & -- & - & - & - & - & - \\
\hline $4 S / 3 W-32 B 1$ & -- & -- & -- & - & - & - & -- & 700 & - \\
\hline \multirow[t]{27}{*}{$5 S / 2 W-19 N 1$} & - & - & -- & - & - & - & -- & 160 & - \\
\hline & - & - & -- & - & -- & - & -- & 150 & -- \\
\hline & - & - & -- & - & -- & - & -- & 0 & - \\
\hline & - & -- & - & - & -- & - & -- & 200 & - \\
\hline & - & - & - & - & - & - & - & 90 & - \\
\hline & - & - & -- & - & - & - & -- & - & - \\
\hline & - & - & -- & - & - & - & - & - & - \\
\hline & - & - & - & -- & - & - & -- & 100 & -- \\
\hline & - & - & -- & -- & - & - & -- & 200 & - \\
\hline & - & -- & - & - & - & - & -- & 100 & - \\
\hline & - & - & - & - & - & - & -- & 60 & - \\
\hline & - & - & - & -- & -. & - & - & 100 & -- \\
\hline & -- & - & - & -- & - & - & - & 300 & - \\
\hline & - & - & -- & -- & - & - & - & 20 & -- \\
\hline & -- & -- & -- & -- & -. & -- & -- & 30 & -. \\
\hline & -- & - & -- & -- & .. & - & -- & 20 & -- \\
\hline & -- & -- & - & -- & -- & - & -- & 10 & -- \\
\hline & - & -- & -- & -- & -. & - & -- & 0 & -- \\
\hline & - & -- & -- & -. & -- & -- & - & 0 & -- \\
\hline & - & - & - & - & -- & -- & - & 20 & -- \\
\hline & - & -- & -- & -- & - & - & - & 60 & -- \\
\hline & -- & -- & -- & - & -- & -- & - & 0 & - \\
\hline & - & -- & - & -- & - & -- & -- & 180 & - \\
\hline & -- & - & - & -- & -- & - & - & 240 & -- \\
\hline & -- & - & - & - & - & - & -- & 0 & - \\
\hline & - & - & -- & -- & -- & -- & -- & 40 & -- \\
\hline & -- & -- & - & -- & -- & - & - & 60 & -- \\
\hline $5 S / 2 W-21 M 1$ & 35 & -- & -- & $<0.4$ & -- & 150 & 4.0 & -- & - \\
\hline \multirow[t]{2}{*}{$5 S / 2 W-21 M 2$} & 140 & - & - & - & - & 190 & 3.0 & - & - \\
\hline & 140 & 0.08 & - & 0.11 & 7.0 & 170 & $<2$ & 230 & -- \\
\hline \multirow[t]{2}{*}{$5 S / 2 W-27 N 1$} & 3.3 & -- & -- & 5.0 & - & $<100$ & $<10$ & - & -- \\
\hline & 6.4 & 0.06 & - & 0.02 & $<1.0$ & -- & -- & 2,500 & - \\
\hline $5 S / 2 W-28 E 1$ & 2.2 & 0.26 & -- & 0.22 & 3.0 & 400 & $<10$ & 100 & - \\
\hline \multirow[t]{3}{*}{$5 S / 2 W-29 N 1$} & $<0.02$ & - & -- & 0.60 & - & $<100$ & $<10$ & - & - \\
\hline & 12 & 0.06 & -- & 0.04 & 1.0 & 23 & $<2$ & 1,300 & - \\
\hline & 13 & 0.03 & - & 0.04 & 1.0 & 23 & $<2$ & 1,400 & - \\
\hline $5 S / 2 W-30 \mathrm{C} 1$ & - & - & -- & $<0.03$ & $<100$ & 50 & $<1$ & $<5$ & $<7$ \\
\hline \multirow[t]{4}{*}{$5 S / 2 W-30 D 2$} & -- & -- & - & -- & - & - & - & 100 & - \\
\hline & - & -- & - & - & -- & 79 & $<2$ & - & - \\
\hline & 12 & 0.06 & - & 0.06 & 1.0 & 74 & $<2$ & 130 & - \\
\hline & 12 & 0.06 & - & 0.07 & 1.0 & 79 & $<2$ & 130 & - \\
\hline $5 \mathrm{~S} / 2 \mathrm{~W}-30 \mathrm{~J} 1$ & - & $<1.0$ & - & - & - & - & - & 700 & - \\
\hline $5 S / 2 W-31 A 1$ & - & - & - & -- & - & - & - & 700 & - \\
\hline $5 S / 2 W-31 N 1$ & -- & -- & -- & - & - & - & - & 50 & - \\
\hline
\end{tabular}


Table 2. Historical water-quality data for selected wells in the Winchester, Menifee, and south Perris ground-water subbasins, California-Continued

\begin{tabular}{|c|c|c|c|c|c|c|c|c|c|}
\hline Well No. & $\begin{array}{c}\text { Chromium, } \\
\text { dissolved } \\
(\mu g / L)\end{array}$ & $\begin{array}{c}\text { Cobalt, } \\
\text { dissolved } \\
(\mu \mathrm{g} / \mathrm{L}) \\
\end{array}$ & $\begin{array}{c}\text { Copper, } \\
\text { dissolved } \\
(\mu \mathrm{g} / \mathrm{L})\end{array}$ & $\begin{array}{c}\text { Iron, } \\
\text { dissolved } \\
(\mu g / L)\end{array}$ & $\begin{array}{c}\text { Lead, } \\
\text { dissolved } \\
(\mu g / L) \\
\end{array}$ & $\begin{array}{l}\text { Lithium, } \\
\text { dissolved } \\
(\mu g / L)\end{array}$ & $\begin{array}{c}\text { Manganese, } \\
\text { dissolved } \\
(\mu g / L) \\
\end{array}$ & $\begin{array}{c}\text { Molybdenum, } \\
\text { dissolved } \\
(\mu \mathrm{g} / \mathrm{L})\end{array}$ & $\begin{array}{c}\text { Nickel, } \\
\text { dissolved } \\
(\mu \mathrm{g} / \mathrm{L})\end{array}$ \\
\hline & -- & -- & -- & -- & -- & -- & -- & -- & - \\
\hline & -- & -- & - & -- & -- & -- & -- & - & -- \\
\hline & -- & -- & -- & -- & -- & - & -- & -- & -- \\
\hline & - & -- & -- & - & -- & -- & -- & - & -- \\
\hline \multirow{2}{*}{$4 \mathrm{~S} / 3 \mathrm{~W}-29 \mathrm{~K} 1$} & - & -- & - & -- & -- & -- & -- & -- & -- \\
\hline & -- & -- & -- & 100 & -- & - & -- & - & -- \\
\hline \multirow[t]{7}{*}{$4 S / 3 W-29 Q 1$} & -- & -- & -- & - & -- & - & -- & - & -- \\
\hline & -- & -- & - & & -- & -- & -- & -- & -- \\
\hline & -- & -- & -- & -- & -- & -- & -- & -- & -- \\
\hline & -- & -- & -- & - & -- & -- & -- & - & -- \\
\hline & - & -- & -- & -- & -- & -- & -- & - & -- \\
\hline & -- & -- & -- & -- & -- & -- & -- & - & -- \\
\hline & -- & -- & -- & 2,000 & -- & -- & 0 & -- & -- \\
\hline $4 S / 3 W-32 B 1$ & -- & -- & -- & - & -- & -- & -- & -- & -- \\
\hline \multirow{27}{*}{$5 \mathrm{~S} / 2 \mathrm{~W}-19 \mathrm{~N} 1$} & -- & -- & - & -- & -- & - & -- & - & - \\
\hline & -- & -- & -- & -- & -- & -- & -- & - & -- \\
\hline & -- & -- & -- & -- & -- & -- & -- & - & -- \\
\hline & -- & -- & - & -- & - & -- & -- & -- & -- \\
\hline & - & -- & -- & -- & -- & -- & -- & -- & -- \\
\hline & -- & -- & - & -- & -- & -- & -- & -- & -- \\
\hline & -- & - & - & -- & -- & -- & -- & -- & -- \\
\hline & -- & - & - & -- & -- & -- & -- & -- & -- \\
\hline & -- & -- & - & - & -- & -- & - & -- & -- \\
\hline & -- & -- & - & -- & -- & -- & -- & -- & -- \\
\hline & - & - & -- & -- & -- & -- & -- & -- & -- \\
\hline & -- & -- & - & -- & -- & - & -- & - & -- \\
\hline & -- & - & - & -- & - & - & - & - & -- \\
\hline & -- & -- & -. & -- & -- & -- & -- & - & -- \\
\hline & -- & -- & -- & -- & - & -- & -- & -- & -- \\
\hline & -- & - & - & -- & - & -- & -- & - & -- \\
\hline & -- & -- & - & - & -- & - & -- & - & -- \\
\hline & -- & -- & - & -- & -- & - & -- & - & -- \\
\hline & -- & - & - & -- & -- & - & -- & -- & -- \\
\hline & -- & -- & -- & -- & -- & -- & -- & -- & -- \\
\hline & -- & -- & - & -- & -- & -- & -- & - & -- \\
\hline & -- & -- & - & - & -- & -- & -- & -- & -- \\
\hline & -- & - & -- & - & -- & -- & -- & - & -- \\
\hline & - & -- & -- & - & -- & -- & -- & -- & -- \\
\hline & - & -- & -- & - & -- & -- & -- & - & -- \\
\hline & -- & -- & -- & -- & -- & -- & -- & -- & -- \\
\hline & -- & -- & -- & -- & -- & - & - & - & -- \\
\hline $5 \mathrm{~S} / 2 \mathrm{~W}-21 \mathrm{M} 1$ & $<20$ & $<9$ & $<30$ & 10 & $<30$ & 110 & $<3$ & $<30$ & $<30$ \\
\hline \multirow{2}{*}{$5 \mathrm{~S} / 2 \mathrm{~W}-21 \mathrm{M} 2$} & $<20$ & $<9$ & $<30$ & 20 & $<30$ & 160 & 320 & 40 & $<30$ \\
\hline & $<20$ & $<9$ & $<30$ & 20 & $<30$ & 170 & 220 & $<30$ & $<30$ \\
\hline \multirow{2}{*}{$5 \mathrm{~S} / 2 \mathrm{~W}-27 \mathrm{~N} 1$} & 3.0 & 2 & 5.0 & 70 & $<1$ & 30 & 100 & 1 & 4.0 \\
\hline & 1.0 & $<1$ & 4.0 & 460 & $<1$ & 30 & 80 & 1 & 4.0 \\
\hline $5 \mathrm{~S} / 2 \mathrm{~W}-28 \mathrm{E} 1$ & 4.0 & 3 & $<1$ & 100 & $<1$ & 190 & 790 & 6 & 5.0 \\
\hline \multirow{3}{*}{$5 \mathrm{~S} / 2 \mathrm{~W}-29 \mathrm{~N} 1$} & 8.0 & $<1$ & 1.0 & $<10$ & $<1$ & 40 & 230 & 14 & 2.0 \\
\hline & $<20$ & $<9$ & $<30$ & $<9$ & $<30$ & 40 & 1,100 & $<30$ & $<30$ \\
\hline & $<20$ & $<9$ & $<30$ & $<9$ & $<30$ & 50 & 1,100 & $<30$ & $<30$ \\
\hline $5 \mathrm{~S} / 2 \mathrm{~W}-30 \mathrm{C} 1$ & $<10$ & $<9$ & $<2$ & 1,600 & $<50$ & 30 & 12 & $<80$ & $<40$ \\
\hline \multirow[t]{4}{*}{$5 \mathrm{~S} / 2 \mathrm{~W}-30 \mathrm{D} 2$} & -- & -- & 10 & 150 & -- & -- & $<10$ & -- & -- \\
\hline & $<20$ & $<9$ & $<30$ & 9 & $<30$ & 30 & 94 & 30 & $<30$ \\
\hline & $<20$ & $<9$ & $<30$ & 10 & $<30$ & 30 & 150 & $<30$ & $<30$ \\
\hline & $<20$ & $<9$ & $<30$ & 10 & $<30$ & 30 & 210 & $<30$ & $<30$ \\
\hline $5 \mathrm{~S} / 2 \mathrm{~W}-30 \mathrm{~J} 1$ & -- & -- & -- & - & -- & -- & -- & - & - \\
\hline $5 \mathrm{~S} / 2 \mathrm{~W}-31 \mathrm{~A} 1$ & -- & -- & -- & - & -- & -- & - & -- & -- \\
\hline $5 \mathrm{~S} / 2 \mathrm{~W}-31 \mathrm{~N} 1$ & -- & -- & -- & -- & -- & -- & -- & -- & -- \\
\hline
\end{tabular}


Table 2. Historical water-quality data for selected wells in the Winchester, Menifee, and south Perris ground-water subbasins, California-Continued

\begin{tabular}{|c|c|c|c|c|c|c|}
\hline Well No. & $\begin{array}{c}\text { Selenium, } \\
\text { dissolved } \\
(\mu \mathrm{g} / \mathrm{L})\end{array}$ & $\begin{array}{c}\text { Silver, } \\
\text { dissolved } \\
(\mu \mathrm{g} / \mathrm{L})\end{array}$ & $\begin{array}{c}\text { Strontium, } \\
\text { dissolved } \\
(\mu \mathrm{g} / \mathrm{L})\end{array}$ & $\begin{array}{c}\text { Vanadium, } \\
\text { dissolved } \\
(\mu \mathrm{g} / \mathrm{L})\end{array}$ & $\begin{array}{c}\text { Zinc, } \\
\text { dissolved } \\
(\mu g / L)\end{array}$ & Source of data \\
\hline & - & - & - & - & -- & RCFCD \\
\hline & - & -- & -- & - & -- & RCFCD \\
\hline & - & -- & -- & - & -- & RCFCD \\
\hline & - & - & - & - & - & EMWD \\
\hline \multirow[t]{2}{*}{$4 S / 3 W-29 K 1$} & -- & -- & -- & - & -- & RCFCD \\
\hline & - & - & - & - & -- & EMWD/Babcock \\
\hline \multirow[t]{7}{*}{$4 S / 3 W-29 Q 1$} & - & - & - & - & - & RCFCD \\
\hline & - & -- & - & - & -- & DPH \\
\hline & -- & -- & - & - & -- & RCFCD \\
\hline & - & -- & -- & - & - & RCFCD \\
\hline & -- & -- & -- & - & - & RCFCD \\
\hline & -- & -- & - & - & - & EMWD \\
\hline & -- & -- & -- & - & - & $\mathrm{DPH}$ \\
\hline 4S/3W-32B1 & -- & - & - & - & - & Babcock \\
\hline \multirow[t]{27}{*}{$5 S / 2 W-19 N 1$} & -- & -- & - & - & -- & RCFCD \\
\hline & -- & - & -- & - & -- & RCFCD \\
\hline & - & - & - & - & -- & RCFCD \\
\hline & -- & - & - & - & -- & RCFCD * \\
\hline & -- & -- & -- & - & - & RCFCD * \\
\hline & -- & -- & - & - & -- & RCFCD * \\
\hline & - & -- & - & - & -- & RCFCD * \\
\hline & - & -- & - & - & -- & RCFCD * \\
\hline & - & - & - & -- & -- & RCFCD * \\
\hline & -- & -- & -- & -- & -- & RCFCD \\
\hline & -- & -- & - & - & - & RCFCD * \\
\hline & - & - & - & -- & - & RCFCD * \\
\hline & -- & -- & - & - & -- & RCFCD * \\
\hline & - & -- & - & - & -- & RCFCD * \\
\hline & - & -- & - & -- & - & RCFCD * \\
\hline & -- & -- & - & - & - & RCFCD * \\
\hline & - & - & -- & - & - & RCFCD * \\
\hline & - & - & -- & -- & -- & RCFCD * \\
\hline & - & - & - & - & -- & RCFCD * \\
\hline & - & - & - & -- & -- & RCFCD * \\
\hline & - & - & - & -- & - & RCFCD \\
\hline & -- & - & - & - & - & RCFCD * \\
\hline & -- & - & -- & - & - & RCFCD * \\
\hline & - & -- & - & -- & - & RCFCD * \\
\hline & - & -- & - & - & - & RCFCD * \\
\hline & -- & -- & - & - & -- & RCFCD * \\
\hline & -- & -- & - & - & - & RCFCD \\
\hline $5 S / 2 W-21 M 1$ & -- & $<3.0$ & 850 & 24 & 250 & USGS \\
\hline \multirow[t]{2}{*}{$5 S / 2 W-21 M 2$} & -- & $<3.0$ & 1,700 & $<18$ & 540 & USGS \\
\hline & $<1$ & $<3.0$ & 1,400 & 19 & 230 & USGS \\
\hline \multirow[t]{2}{*}{$5 S / 2 W-27 N 1$} & -- & $<1.0$ & 1,700 & - & 110 & USGS \\
\hline & 6.0 & $<1.0$ & - & 37 & 90 & USGS \\
\hline $5 S / 2 W-28 E 1$ & - & $<1.0$ & 4,100 & 120 & 180 & USGS \\
\hline \multirow[t]{3}{*}{$5 \mathrm{~S} / 2 \mathrm{~W}-29 \mathrm{~N} 1$} & -- & $<1.0$ & 3,300 & - & 30 & USGS \\
\hline & 7.0 & $<3.0$ & 2,600 & 21 & 10 & USGS \\
\hline & - & $<3.0$ & 2,800 & $<18$ & $<9$ & USGS \\
\hline $5 S / 2 W-30 C 1$ & $<100$ & $<0.003$ & 200 & $<3$ & 110 & Vetter Research \\
\hline \multirow[t]{4}{*}{$5 S / 2 W-30 D 2$} & $<5$ & - & - & - & $<10$ & Babcock \\
\hline & - & $<3.0$ & 2,200 & $<18$ & 10 & USGS \\
\hline & 6.0 & $<3.0$ & 2,100 & $<18$ & 30 & USGS \\
\hline & - & $<3.0$ & 2,400 & $<18$ & $<9$ & USGS \\
\hline $5 S / 2 W-30 J 1$ & - & - & - & - & - & EMWD \\
\hline $5 S / 2 W-31 A 1$ & - & - & - & -- & -- & EMWD \\
\hline $5 S / 2 W-31 N 1$ & - & - & - & - & - & DWR \\
\hline
\end{tabular}


Table 2. Historical water-quality data for selected wells in the Winchester, Menifee, and south Perris ground-water subbasins, California-Continued

\begin{tabular}{|c|c|c|c|c|c|c|c|c|}
\hline \multirow[t]{2}{*}{ Well No. } & \multirow[t]{2}{*}{ Date } & \multirow{2}{*}{$\begin{array}{l}\text { Water level } \\
\text { (ft blw LSD) }\end{array}$} & \multirow{2}{*}{$\begin{array}{c}\text { Well depth } \\
\text { (ft) }\end{array}$} & \multirow{2}{*}{$\begin{array}{c}\text { Altitude } \\
\text { of LSD (ft) }\end{array}$} & \multicolumn{2}{|c|}{ Specific conductance $(\mu \mathrm{S} / \mathrm{cm})$} & \multirow{2}{*}{$\begin{array}{c}\text { pH (lab) } \\
\text { (Std. units) }\end{array}$} & \multirow{2}{*}{$\begin{array}{c}\text { Water } \\
\text { temperature } \\
\left({ }^{\circ} \mathrm{C}\right)\end{array}$} \\
\hline & & & & & Fieid & Lab & & \\
\hline $5 S / 2 W-31 R 1$ & $7 / 16 / 87$ & - & - & - & - & 525 & 7.4 & - \\
\hline \multirow[t]{2}{*}{$5 S / 2 W-33 E 1$} & $2 / 26 / 81$ & - & - & - & - & 1,900 & 7.1 & - \\
\hline & $5 / 11 / 81$ & - & & & - & 1,400 & - & -- \\
\hline $5 S / 3 W-2 Q 1$ & $11 / 19 / 93$ & - & - & 1,520 & 671 & 674 & 7.8 & 22.0 \\
\hline $5 S / 3 W-3 C 1$ & $11 / 2 / 93$ & - & 250.8 & 1,420 & - & 7,400 & 7.3 & -- \\
\hline $5 S / 3 W-3 L 1$ & $10 / 29 / 93$ & -- & 252 & 1,425 & - & 3,300 & 7.6 & - \\
\hline $5 S / 3 W-3 N 1$ & $10 / 22 / 93$ & - & 577.8 & 1,415 & -- & 4,700 & 6.8 & - \\
\hline $5 S / 3 W-3 Q 2$ & $5 / 22 / 75$ & - & - & - & - & 903 & 7.7 & -- \\
\hline $5 S / 3 W-3 R 2$ & $4 / 18 / 77$ & -- & -- & - & - & -- & - & -- \\
\hline $5 S / 3 W-4 A 1$ & $11 / 5 / 93$ & -- & 209.4 & 1,415 & - & 3,800 & 7.6 & - \\
\hline $5 S / 3 W-4 M 1$ & $10 / 15 / 93$ & - & 251.3 & 1,412 & - & 18,000 & 7.0 & - \\
\hline $5 S / 3 W-9 E 1$ & $10 / 26 / 93$ & -- & 238.4 & 1,415 & - & 3,100 & 7.7 & - \\
\hline $5 \mathrm{~S} / 3 \mathrm{~W}-9 \mathrm{H} 2$ & $10 / 21 / 93$ & - & 250.6 & 1,420 & - & 2,650 & 6.6 & - \\
\hline $5 S / 3 W-9 Q 1$ & $6 / 7 / 83$ & -- & 600 & 1,421 & - & 1,760 & 6.7 & 26.5 \\
\hline 5S/3W-11M1 & $12 / 18 / 53$ & - & 615 & 1,451 & - & 448 & 7.6 & 24.0 \\
\hline \multirow[t]{18}{*}{$5 S / 3 W-11 M 2$} & $7 / 28 / 55$ & - & 787 & 1,451 & - & 1,230 & - & 23.5 \\
\hline & $9 / 18 / 56$ & - & & & -- & 1,360 & - & 23.0 \\
\hline & $9 / 17 / 57$ & -- & & & -- & 1,380 & 8.1 & 22.0 \\
\hline & $10 / 14 / 58$ & - & & & - & 1,430 & 6.8 & 24.5 \\
\hline & $4 / 7 / 59$ & - & & & - & 1,440 & 6.5 & 23.5 \\
\hline & $9 / 14 / 60$ & -- & & & - & 1,420 & 6.5 & 23.5 \\
\hline & $9 / 28 / 61$ & - & & & -- & 1,510 & 7.1 & 23.5 \\
\hline & $9 / 21 / 62$ & - & & & - & 1,100 & 6.9 & 22.0 \\
\hline & $9 / 27 / 63$ & - & & & - & 1,430 & 6.6 & 24.5 \\
\hline & $4 / 16 / 64$ & - & & & - & 1,490 & 7.1 & 23.5 \\
\hline & $4 / 28 / 70$ & -- & & & -- & 1,770 & 8.1 & 22.0 \\
\hline & $11 / 4 / 71$ & - & & & -- & 1,520 & 7.9 & 23.5 \\
\hline & $5 / 11 / 72$ & - & & & - & 1,520 & 8.4 & 24.0 \\
\hline & $9 / 27 / 73$ & - & & & - & 1,190 & 8.1 & 23.5 \\
\hline & $5 / 16 / 75$ & -- & & & -- & 1,400 & 6.5 & - \\
\hline & $4 / 22 / 77$ & -- & & & - & 1,500 & 7.2 & 24.5 \\
\hline & $5 / 11 / 81$ & - & & & - & 1,450 & 6.8 & 23.0 \\
\hline & $6 / 7 / 83$ & - & & & - & 1,280 & 7.0 & 24.0 \\
\hline \multirow[t]{3}{*}{$5 S / 3 W-13 A 1$} & $9 / 17 / 77$ & -- & & & - & 600 & 7.6 & 23.5 \\
\hline & $5 / 11 / 81$ & - & & & - & 625 & 7.9 & 23.5 \\
\hline & $11 / 18 / 93$ & - & 431 & 1,522 & 890 & 897 & 7.5 & 22.0 \\
\hline \multirow[t]{2}{*}{$5 \mathrm{~S} / 3 \mathrm{~W}-13 \mathrm{H} 1$} & $11 / 18 / 93$ & -- & 460 & 1,518 & 1,160 & 1,170 & 7.4 & 21.5 \\
\hline & $1 / 25 / 94$ & -- & & & 1,110 & 1,110 & 7.5 & 22.0 \\
\hline \multirow[t]{3}{*}{$5 S / 3 W-14 P 1$} & $5 / 30 / 75$ & - & 250 & 1,447 & - & 1,270 & 8.0 & 23.0 \\
\hline & $4 / 22 / 77$ & - & & & -- & - & - & - \\
\hline & 7/9/85 & -- & & & -- & 1,570 & 6.4 & - \\
\hline $5 \mathrm{~S} / 3 \mathrm{~W}-15 \mathrm{H} 1$ & $11 / 19 / 93$ & - & 220 & 1,435 & 1,500 & 1,520 & 7.2 & 21.5 \\
\hline $5 S / 3 W-16 D 1$ & $11 / 18 / 93$ & - & 160 & 1,417 & 3,300 & 3,320 & 7.6 & 20.0 \\
\hline $5 S / 3 W-16 F 1$ & $11 / 18 / 93$ & - & - & 1,419 & 1,400 & 1,420 & 7.6 & 21.0 \\
\hline \multirow[t]{6}{*}{$5 S / 3 W-16 P 1$} & $8 / 9 / 55$ & -- & - & - & - & 1,660 & - & 22.0 \\
\hline & $5 / 9 / 56$ & - & & & - & 1,490 & - & 21.0 \\
\hline & $9 / 18 / 56$ & - & & & - & 1,540 & - & 22.0 \\
\hline & $5 / 27 / 57$ & - & & & - & 1,410 & 7.5 & 20.5 \\
\hline & $9 / 27 / 57$ & - & & & - & 1,340 & 6.9 & 21.5 \\
\hline & $5 / 8 / 58$ & - & & & - & 1,290 & 6.6 & 22.0 \\
\hline \multirow[t]{3}{*}{$5 S / 3 W-16 P 2$} & $4 / 18 / 77$ & - & 568 & 1,425 & - & 2,290 & 6.5 & 24.0 \\
\hline & $5 / 11 / 81$ & - & & & - & 2,900 & 6.7 & 23.5 \\
\hline & $6 / 7 / 83$ & - & & & - & 3,100 & 6.9 & - \\
\hline $5 S / 3 W-17 R 1$ & $8 / 30 / 91$ & -- & 370 & 1,445 & - & 2,100 & 7.7 & - \\
\hline $5 S / 3 W-17 R 2$ & $1 / 26 / 95$ & -. & 220 & 1,460 & -- & - & - & - \\
\hline $5 S / 3 W-21 C 1$ & $11 / 7 / 75$ & -- & -- & - & -- & 4,500 & 6.9 & - \\
\hline & $4 / 19 / 77$ & -- & & & - & - & -- & - \\
\hline 5S/3W-21D1 & $9 / 25 / 62$ & - & 356 & - & -- & 630 & 7.7 & 23.5 \\
\hline
\end{tabular}


Table 2. Historical water-quality data for selected wells in the Winchester, Menifee, and south Perris ground-water subbasins, California-Continued

\begin{tabular}{|c|c|c|c|c|c|c|c|c|}
\hline \multirow[t]{2}{*}{ Well No. } & \multirow{2}{*}{$\begin{array}{c}\text { Hardness } \\
\left(\mathrm{mg} / \mathrm{L} \text { as } \mathrm{CaCO}_{3}\right)\end{array}$} & \multirow{2}{*}{$\begin{array}{c}\text { Calcium, } \\
\text { dissolved } \\
(\mathrm{mg} / \mathrm{L}) \\
\end{array}$} & \multirow{2}{*}{$\begin{array}{c}\text { Magnesium, } \\
\text { dissolved } \\
(\mathrm{mg} / \mathrm{L})\end{array}$} & \multirow{2}{*}{$\begin{array}{c}\text { Sodium, } \\
\text { dissolved } \\
(\mathrm{mg} / \mathrm{L})\end{array}$} & \multirow{2}{*}{$\begin{array}{c}\text { Potassium, } \\
\text { dissolved } \\
\text { (mg/L as K) }\end{array}$} & \multirow{2}{*}{$\begin{array}{c}\text { Bicarbonate } \\
\left(\mathrm{mg} / \mathrm{L} \text { as } \mathrm{HCO}_{3}\right) \\
\end{array}$} & \multicolumn{2}{|c|}{ Alkalinity $\left(\mathrm{mg} / \mathrm{L}\right.$ as $\left.\mathrm{CaCO}{ }_{3}\right)$} \\
\hline & & & & & & & Field & Lab \\
\hline $5 S / 2 W-31 R 1$ & 170 & - & -- & 52.0 & 6 & - & - & - \\
\hline \multirow[t]{2}{*}{$5 S / 2 W-33 E 1$} & 500 & 120 & 47.0 & 200.0 & 3 & 150 & - & - \\
\hline & - & -- & -- & - & -- & -- & - & - \\
\hline $5 S / 3 W-3 L 1$ & 1,100 & 350 & 56.0 & 270.0 & 12 & 210 & - & 180 \\
\hline $5 S / 3 W-3 N 1$ & 1,800 & 500 & 120.0 & 260.0 & 13 & 380 & - & 310 \\
\hline $5 S / 3 W-3 Q 2$ & 180 & 59 & 7.5 & 96.0 & 4 & 73 & - & 60 \\
\hline $5 S / 3 W-3 R 2$ & -- & -- & -- & -- & -- & - & -- & - \\
\hline $5 S / 3 W-4 A 1$ & 500 & 150 & 27.0 & 620.0 & 9 & 180 & - & 150 \\
\hline $5 S / 3 W-4 M 1$ & 7,300 & 2,220 & 410.0 & 1300.0 & 26 & 270 & - & 220 \\
\hline \multirow[t]{12}{*}{$5 S / 3 W-11 M 2$} & - & 100 & 35.0 & 98.0 & - & 290 & - & -- \\
\hline & 430 & 110 & 36.0 & 100.0 & - & 290 & - & -- \\
\hline & 470 & 130 & 38.0 & 99.0 & - & 180 & - & -- \\
\hline & 480 & 130 & 36.0 & 99.0 & - & 160 & - & -- \\
\hline & 500 & 150 & 32.0 & 98.0 & - & 260 & - & -- \\
\hline & 520 & 170 & 21.0 & 90.0 & 12 & 260 & - & -- \\
\hline & 480 & 160 & 21.0 & 89.0 & 14 & 240 & - & - \\
\hline & 380 & 110 & 27.0 & 70.0 & 8 & 170 & - & -- \\
\hline & 470 & 140 & 28.0 & 90.0 & 10 & 240 & - & 200 \\
\hline & 470 & 140 & 30.0 & 84.0 & 12 & 250 & - & 210 \\
\hline & 660 & 170 & 54.0 & 96.0 & 11 & 290 & - & 240 \\
\hline & 510 & 140 & 41.0 & 86.0 & 9 & 260 & - & 210 \\
\hline $5 S / 3 W-13 A 1$ & 270 & 70 & 23.0 & 72.0 & 5 & $\ldots$ & 180 & - \\
\hline \multirow[t]{2}{*}{$5 \mathrm{~S} / 3 \mathrm{~W}-13 \mathrm{H} 1$} & 370 & 96 & 31.0 & 87.0 & 6 & - & 180 & - \\
\hline & 330 & 87 & 27.0 & 78.0 & 6 & - & 160 & -- \\
\hline \multirow[t]{3}{*}{$5 \mathrm{~S} / 3 \mathrm{~W}-14 \mathrm{P} 1$} & 410 & 110 & 31.0 & 75.0 & 3 & 150 & - & 120 \\
\hline & - & - & -- & - & -- & - & - & - \\
\hline & 570 & 160 & 44.0 & 93.0 & 4 & 240 & - & 190 \\
\hline $5 \mathrm{~S} / 3 \mathrm{~W}-15 \mathrm{H} 1$ & 520 & 140 & 42.0 & 84.0 & 3 & - & 150 & -- \\
\hline $5 S / 3 W-16 D 1$ & 810 & 230 & 56.0 & 360.0 & 5 & - & 290 & - \\
\hline $5 S / 3 W-16 F 1$ & 420 & 120 & 28.0 & 100.0 & 3 & - & 110 & -- \\
\hline \multirow[t]{6}{*}{$5 S / 3 W-16 P 1$} & 690 & 170 & 61.0 & 63.0 & -- & 290 & - & - \\
\hline & 540 & 150 & 38.0 & 68.0 & - & 350 & - & -- \\
\hline & 580 & 150 & 47.0 & 63.0 & -- & 300 & - & -- \\
\hline & 560 & 140 & 51.0 & 61.0 & - & 310 & - & - \\
\hline & 540 & 150 & 41.0 & 58.0 & - & 320 & -- & 260 \\
\hline & 560 & 150 & 47.0 & 60.0 & -- & 410 & -- & - \\
\hline \multirow[t]{3}{*}{$5 S / 3 W-16 P 2$} & 1,100 & 290 & 81.0 & 100.0 & 7 & 540 & - & - \\
\hline & 1,200 & 350 & 76.0 & 140.0 & 8 & 390 & - & -- \\
\hline & 1,600 & 430 & 110.0 & 150.0 & 9 & 780 & & 780 \\
\hline 5S/3W-17R1 & 900 & 200 & 99.0 & 73.0 & 5 & 210 & - & 170 \\
\hline 5S/3W-17R2 & - & - & -- & - & - & - & - & - \\
\hline $5 S / 3 W-21 C 1$ & 1,500 & 470 & 90.0 & 190.0 & 12 & 390 & - & -- \\
\hline & - & -- & -- & - & - & - & - & - \\
\hline 5S/3W-21D1 & 180 & 61 & 6.0 & 56.0 & 3 & 120 & - & - \\
\hline
\end{tabular}


Table 2. Historical water-quality data for selected wells in the Winchester, Menifee, and south Perris ground-water subbasins, California-Continued

\begin{tabular}{|c|c|c|c|c|c|c|c|c|c|}
\hline \multirow[t]{2}{*}{ Well No. } & \multirow{2}{*}{$\begin{array}{c}\text { Sulfate, } \\
\text { dissolved } \\
\left(\mathrm{mg} / \mathrm{L} \text { as } \mathrm{SO}_{4}\right)\end{array}$} & \multirow{2}{*}{$\begin{array}{c}\text { Chloride, } \\
\text { dissolved } \\
(\mathrm{mg} / \mathrm{L})\end{array}$} & \multirow{2}{*}{$\begin{array}{c}\text { Fluoride, } \\
\text { dissolved } \\
(\mathrm{mg} / \mathrm{L})\end{array}$} & \multirow{2}{*}{$\begin{array}{c}\text { Bromide, } \\
\text { dissolved } \\
(\mathrm{mg} / \mathrm{L})\end{array}$} & \multirow{2}{*}{$\begin{array}{c}\text { Silica, } \\
\text { dissolved } \\
\left(\mathrm{mg} / \mathrm{L} \text { as } \mathrm{SiO}_{2}\right)\end{array}$} & \multicolumn{2}{|c|}{ Dissolved solids (mg/L) } & \multirow{2}{*}{$\begin{array}{c}\text { Nitrate, } \\
\text { dissolved } \\
\text { (mg/L as } \mathrm{N} \text { ) } \\
\end{array}$} & \multirow{2}{*}{$\begin{array}{c}\text { Nitrite } \\
\text { dissolved } \\
\text { (mg/L as N) }\end{array}$} \\
\hline & & & & & & (sum) & (residue) & & \\
\hline $5 S / 2 W-31 R 1$ & - & -- & 0.5 & - & - & 310 & - & 3.8 & - \\
\hline \multirow[t]{2}{*}{$5 S / 2 W-33 E 1$} & 150 & 440 & 0.5 & -- & - & - & 1,240 & 9.6 & - \\
\hline & -- & - & - & -- & - & 1,010 & -- & - & - \\
\hline $5 S / 3 W-2 Q 1$ & -- & -- & - & -- & 32 & - & - & - & $<0.010$ \\
\hline $5 S / 3 W-3 C 1$ & 110 & 2,400 & 0.1 & -- & - & -- & 5,090 & 2.9 & $<0.10$ \\
\hline $5 S / 3 W-3 L 1$ & 34 & 1,040 & 0.2 & -- & - & - & 2,710 & 5.0 & $<0.10$ \\
\hline $5 S / 3 W-3 N 1$ & 58 & 1,400 & 0.5 & - & - & -- & 3,490 & 1.4 & $<0.10$ \\
\hline $5 S / 3 W-3 Q 2$ & 24 & 220 & 0.7 & -- & -- & -- & 640 & 1.9 & -- \\
\hline $5 S / 3 W-3 R 2$ & - & - & - & - & -- & -. & 1,280 & - & - \\
\hline $5 S / 3 W-4 A 1$ & 240 & 1,100 & 0.2 & -- & -- & - & 2,260 & 3.4 & $<0.10$ \\
\hline $5 S / 3 W-4 M 1$ & 370 & 6,700 & 0.1 & -- & -- & -- & 14,000 & 1.8 & $<0.10$ \\
\hline $5 S / 3 W-9 E 1$ & 150 & 780 & 0.3 & -- & -- & - & 1,970 & 8.6 & $<0.10$ \\
\hline $5 \mathrm{~S} / 3 \mathrm{~W}-9 \mathrm{H} 2$ & 62 & 660 & 0.2 & - & -- & - & 1,860 & 7.5 & $<0.10$ \\
\hline $5 S / 3 W-9 Q 1$ & 41 & 270 & -- & -- & 58 & - & 1,220 & 0.45 & -- \\
\hline $5 S / 3 W-11 M 1$ & 16 & 70 & 0.5 & -- & - & -- & 260 & 3.6 & - \\
\hline \multirow[t]{18}{*}{$5 S / 3 W-11 M 2$} & 100 & 190 & 0.2 & -- & - & 680 & - & 2.0 & -- \\
\hline & 39 & 280 & 0.0 & -- & - & 730 & -- & 1.6 & - \\
\hline & 35 & 310 & 0.2 & -- & -- & 760 & - & 1.6 & - \\
\hline & 40 & 320 & 0.0 & - & - & 770 & -- & 2.7 & - \\
\hline & 40 & 320 & 0.0 & -- & -- & 780 & -- & 1.1 & - \\
\hline & 30 & 340 & 0.0 & - & -- & 800 & - & 1.8 & -- \\
\hline & 80 & 280 & 0.1 & -- & -- & 780 & - & 2.3 & - \\
\hline & 48 & 240 & - & - & - & - & 680 & 1.4 & - \\
\hline & 28 & 310 & 0.1 & -- & -- & -- & 870 & 2.7 & - \\
\hline & 45 & 280 & 0.1 & - & - & - & 860 & 3.6 & - \\
\hline & 48 & 390 & 0.2 & - & -- & - & 1,190 & 5.2 & - \\
\hline & 43 & 310 & 0.2 & -- & -- & - & 990 & 4.8 & - \\
\hline & 43 & 310 & 0.3 & - & - & -- & 990 & 3.8 & - \\
\hline & 38 & 230 & 0.2 & - & -- & - & 1,000 & 5.2 & - \\
\hline & 43 & 300 & 0.1 & - & -- & - & 950 & 1.0 & - \\
\hline & 45 & 340 & -- & -- & 24 & - & 1,040 & 5.4 & - \\
\hline & 51 & 320 & 0.4 & -- & -- & - & 980 & 6.1 & - \\
\hline & 39 & 290 & - & -- & 50 & - & 920 & 1.0 & - \\
\hline \multirow[t]{3}{*}{$5 S / 3 W-13 A 1$} & 27 & 73 & 0.6 & - & - & - & 400 & 5.7 & -- \\
\hline & 26 & 79 & 0.7 & -- & - & - & 400 & 7.5 & - \\
\hline & 55 & 130 & - & - & 46 & -- & - & 13 & $<0.010$ \\
\hline \multirow[t]{2}{*}{$5 \mathrm{~S} / 3 \mathrm{~W}-13 \mathrm{H} 1$} & 80 & 180 & - & - & 48 & - & - & - & $<0.010$ \\
\hline & 83 & 180 & - & - & 43 & - & -- & -- & 0.03 \\
\hline \multirow[t]{3}{*}{$5 S / 3 W-14 P 1$} & 38 & 280 & 0.3 & - & -- & - & 1,120 & 6.1 & - \\
\hline & -- & -- & -- & - & -- & - & 840 & - & - \\
\hline & 470 & 60 & - & - & - & - & 1,260 & 6.3 & -- \\
\hline $5 \mathrm{~S} / 3 W-15 \mathrm{H} 1$ & 73 & 340 & - & -- & 55 & - & -- & 12 & 0.20 \\
\hline $5 S / 3 W-16 D 1$ & 270 & 740 & -- & - & 52 & - & - & 19 & $<0.010$ \\
\hline $5 S / 3 W-16 F 1$ & 37 & 340 & - & - & 41 & -- & - & 11 & 0.20 \\
\hline $5 S / 3 W-16 P 1$ & 74 & 280 & 0.1 & - & - & - & - & 1.8 & - \\
\hline & 50 & 220 & 0.0 & - & -- & - & -- & 2.9 & - \\
\hline & 76 & 300 & 0.0 & - & - & - & - & 1.6 & - \\
\hline & 70 & 270 & 0.1 & -- & - & -- & - & 1.8 & - \\
\hline & 55 & 260 & 0.0 & - & - & 730 & - & 2.5 & -- \\
\hline & 50 & 210 & 0.0 & - & - & - & - & 3.2 & -- \\
\hline $5 S / 3 W-16 P 2$ & 120 & 490 & 0.2 & - & & - & 1,590 & 5.7 & - \\
\hline & 180 & 680 & 0.3 & -- & -- & - & 2,150 & 7.2 & - \\
\hline & 240 & 450 & -- & - & 54 & - & 2,790 & 0.38 & - \\
\hline $5 S / 3 W-17 R 1$ & 200 & 470 & 0.2 & - & 59 & - & 1,430 & 5.7 & - \\
\hline $5 S / 3 W-17 R 2$ & - & -- & 0.4 & -- & -- & - & 530 & 13 & -- \\
\hline $5 S / 3 W-21 C 1$ & 150 & 1,060 & 5.0 & -- & - & - & 3,060 & 7.0 & -- \\
\hline & - & -- & - & - & - & -- & 2,950 & - & - \\
\hline $5 S / 3 W-21 D 1$ & 50 & 82 & 0.2 & - & - & - & 420 & 9.5 & - \\
\hline
\end{tabular}


Table 2. Historical water-quality data for selected wells in the Winchester, Menifee, and south Perris ground-water subbasins, California--Continued

\begin{tabular}{|c|c|c|c|c|c|c|c|c|c|}
\hline Well No. & $\begin{array}{c}\mathrm{NO}_{3}+\mathrm{NO}_{2} \\
\text { dissolved } \\
(\mathrm{mg} / \mathrm{L} \text { as } \mathrm{N})\end{array}$ & $\begin{array}{c}\text { Nitrogen } \\
\text { ammonia, } \\
\text { dissolved } \\
\text { (mg/L as } \mathrm{N} \text { ) }\end{array}$ & $\begin{array}{l}\text { Phosphorus, } \\
\text { dissolved } \\
\text { (mg/L) }\end{array}$ & $\begin{array}{l}\text { Phosphorus } \\
\text { ortho, } \\
\text { dissolved } \\
\text { (mg/L as } \mathrm{P} \text { ) }\end{array}$ & $\begin{array}{l}\text { Arsenic, } \\
\text { dissolved } \\
(\mu g / L)\end{array}$ & $\begin{array}{c}\text { Barium, } \\
\text { dissolved } \\
(\mu \mathrm{g} / \mathrm{L})\end{array}$ & $\begin{array}{c}\text { Beryllium, } \\
\text { dissolved } \\
(\mu g / L)\end{array}$ & $\begin{array}{c}\text { Boron, } \\
\text { dissolved } \\
(\mu \mathrm{g} / \mathrm{L})\end{array}$ & $\begin{array}{c}\text { Cadmium, } \\
\text { dissolved } \\
(\mu g / L)\end{array}$ \\
\hline $5 S / 2 W-31 R 1$ & - & - & -- & - & - & - & -- & 100 & - \\
\hline \multirow[t]{2}{*}{$5 S / 2 W-33 E 1$} & - & - & -- & -- & - & - & - & 300 & - \\
\hline & -- & -- & -- & -. & - & - & -- & -- & - \\
\hline $5 S / 3 W-2 Q 1$ & 13 & 0.02 & - & 0.15 & 2.0 & 58 & 0.70 & 160 & $<1$ \\
\hline $5 S / 3 W-3 C 1$ & -- & -- & -- & - & $<5$ & 200 & - & - & $<1$ \\
\hline $5 S / 3 W-3 L 1$ & - & - & -- & -- & $<5$ & 600 & - & - & $<1$ \\
\hline $5 S / 3 W-3 N 1$ & -- & -- & - & -- & $<5$ & 400 & - & -- & $<1$ \\
\hline $5 S / 3 W-3 Q 2$ & -- & - & -- & -- & -- & -- & -- & 200 & - \\
\hline $5 S / 3 W-3 R 2$ & - & - & $\ldots$ & -- & -- & -- & - & - & - \\
\hline $5 S / 3 W-4 A 1$ & -- & -- & -- & -- & $<5$ & $<100$ & - & -- & $<1$ \\
\hline $5 S / 3 W-4 M 1$ & - & -- & - & -- & $<5$ & 200 & -- & - & 2.0 \\
\hline $5 S / 3 W-9 E 1$ & - & - & -- & - & $<5$ & 200 & - & - & $<1$ \\
\hline $5 \mathrm{~S} / 3 \mathrm{~W}-9 \mathrm{H} 2$ & - & - & - & -- & $<5$ & 200 & - & - & $<1$ \\
\hline $5 S / 3 W-9 Q 1$ & -- & 0.06 & -- & 11 & -- & - & - & 150 & -- \\
\hline $5 S / 3 W-11 M 1$ & - & - & -- & - & -- & -- & - & 60 & -- \\
\hline \multirow{18}{*}{$5 S / 3 W-11 M 2$} & - & - & -- & - & - & - & - & 90 & - \\
\hline & -- & - & -- & -- & - & - & - & 100 & -- \\
\hline & - & -- & -- & - & -- & - & -- & 90 & -- \\
\hline & -- & - & -- & -- & -- & -- & -- & 150 & - \\
\hline & - & - & - & -- & -- & - & - & - & -- \\
\hline & -- & - & -- & -- & -- & -- & - & 100 & -- \\
\hline & -- & - & - & -- & -- & -- & - & 200 & - \\
\hline & - & -- & - & -- & -- & -- & -- & - & - \\
\hline & -- & - & - & -- & -- & -- & - & 100 & - \\
\hline & -- & - & - & -- & -- & - & - & 100 & - \\
\hline & - & - & - & -- & -- & - & -- & 40 & - \\
\hline & - & -- & -- & -- & - & - & - & 90 & -- \\
\hline & - & -- & -- & - & - & - & - & 170 & - \\
\hline & - & -- & -- & -- & - & - & - & 0 & - \\
\hline & -- & $<1.0$ & - & - & - & - & - & 200 & - \\
\hline & -- & 0.00 & - & 0.10 & - & -- & - & 0 & -- \\
\hline & -- & -- & -- & -- & - & - & - & 500 & - \\
\hline & - & 0.06 & -- & 9.0 & -- & - & - & 20 & - \\
\hline \multirow[t]{3}{*}{$5 S / 3 W-13 A 1$} & - & - & - & - & - & - & - & 200 & -- \\
\hline & -- & -- & -- & -- & -- & - & -- & 300 & - \\
\hline & 13 & 0.04 & -- & 0.02 & 4.0 & 260 & $<0.5$ & 70 & - \\
\hline \multirow[t]{2}{*}{$5 S / 3 W-13 H 1$} & 13 & 0.02 & -- & 0.02 & 4.0 & 200 & $<0.5$ & 60 & - \\
\hline & 14 & 0.02 & -- & 0.02 & 5.0 & 190 & $<0.5$ & 60 & - \\
\hline \multirow[t]{3}{*}{$5 S / 3 W-14 P 1$} & -- & - & - & - & -- & - & -- & 60 & -- \\
\hline & - & - & - & -- & - & - & - & - & -- \\
\hline & - & - & -- & -- & -- & - & -- & 100 & -- \\
\hline $5 S / 3 W-15 H 1$ & 12 & 0.03 & -- & 0.05 & 1.0 & 140 & $<0.5$ & 70 & -- \\
\hline $5 S / 3 W-16 D 1$ & 19 & 0.03 & - & 0.02 & 1.0 & 66 & $<0.5$ & 1,500 & -- \\
\hline $5 S / 3 W-16 F 1$ & 12 & 0.03 & - & 0.02 & $<1$ & 180 & $<0.5$ & 80 & -- \\
\hline \multirow[t]{6}{*}{$5 S / 3 W-16 P 1$} & - & - & -- & - & - & - & - & - & -- \\
\hline & -- & - & -- & - & -- & -- & -- & 40 & - \\
\hline & -- & -- & -- & $\cdots$ & - & - & -- & -- & -- \\
\hline & -- & -- & -- & -- & - & - & -- & 550 & -- \\
\hline & - & -- & -- & -- & -- & - & - & 200 & - \\
\hline & - & -- & -- & -- & - & -- & - & 300 & - \\
\hline \multirow[t]{3}{*}{$5 S / 3 W-16 P 2$} & - & -- & - & - & - & -- & - & 400 & - \\
\hline & - & -- & - & - & -- & -- & - & 700 & - \\
\hline & - & 0.05 & -- & 10 & -- & -. & - & 120 & -- \\
\hline 5S/3W-17R1 & - & -- & -- & - & $<10$ & 100 & -- & $<100$ & $<1$ \\
\hline $5 S / 3 W-17 R 2$ & -- & $\ldots$ & - & - & - & -- & - & - & - \\
\hline \multirow[t]{2}{*}{$5 S / 3 W-21 C 1$} & - & - & - & - & - & -- & -- & 300 & -- \\
\hline & - & -- & -- & - & -- & - & -- & -- & - \\
\hline 5S/3W-21D1 & - & -- & - & - & - & -- & - & 100 & - \\
\hline
\end{tabular}


Table 2. Historical water-quality data for selected wells in the Winchester, Menifee, and south Perris ground-water subbasins, California-Continued

\begin{tabular}{|c|c|c|c|c|c|c|c|c|c|}
\hline Well No. & $\begin{array}{c}\text { Chromium, } \\
\text { dissolved } \\
(\mu g / L)\end{array}$ & $\begin{array}{l}\text { Cobalt, } \\
\text { dissolved } \\
(\mu g / L)\end{array}$ & $\begin{array}{l}\text { Copper, } \\
\text { dissolved } \\
(\mu g / L) \\
\end{array}$ & $\begin{array}{c}\text { Iron, } \\
\text { dissolved } \\
(\mu g / L) \\
\end{array}$ & $\begin{array}{l}\text { Lead, } \\
\text { dissolved } \\
(\mu g / L) \\
\end{array}$ & $\begin{array}{l}\text { Lithium, } \\
\text { dissolved } \\
(\mu \mathrm{g} / \mathrm{L})\end{array}$ & $\begin{array}{c}\text { Manganese, } \\
\text { dissolved } \\
(\mu g / L)\end{array}$ & $\begin{array}{l}\text { Molybdenum, } \\
\text { dissolved } \\
(\mu \mathrm{g} / \mathrm{L})\end{array}$ & $\begin{array}{c}\text { Nickel, } \\
\text { dissolved } \\
(\mu g / L) \\
\end{array}$ \\
\hline $5 \mathrm{~S} / 2 \mathrm{~W}-31 \mathrm{R} 1$ & -- & -- & -- & 50 & -- & -- & 50 & - & - \\
\hline \multirow[t]{2}{*}{ 5S/2W-33E1 } & -- & -- & -- & -- & -- & -- & -- & -- & -- \\
\hline & -- & -- & -- & -- & -- & -- & -- & -- & - \\
\hline $5 S / 3 W-2 Q 1$ & $<5$ & $<3$ & $<10$ & $<3$ & $<10$ & 10 & $<1$ & 20 & $<10$ \\
\hline $5 S / 3 W-3 C 1$ & $<10$ & -- & $<10$ & $<20$ & $<5$ & -- & 30 & -- & -- \\
\hline $5 S / 3 W-3 L 1$ & $<10$ & -- & $<10$ & $<20$ & $<5$ & -- & 40 & -- & .- \\
\hline $5 S / 3 W-3 N 1$ & $<10$ & -- & $<10$ & 450 & $<5$ & -- & 140 & -- & -- \\
\hline $5 S / 3 W-3 Q 2$ & -- & -- & -- & -- & -- & -- & -. & -. & -- \\
\hline $5 S / 3 W-3 R 2$ & -- & -- & -- & -- & -- & -- & -- & -- & -- \\
\hline $5 S / 3 W-4 A 1$ & $<10$ & -- & $<10$ & $<20$ & $<5$ & -- & $<10$ & - & -- \\
\hline $5 \mathrm{~S} / 3 \mathrm{~W}-4 \mathrm{M} 1$ & $<10$ & -- & $<10$ & 400 & $<5$ & -- & 80 & -- & -- \\
\hline $5 S / 3 W-9 E 1$ & $<10$ & -- & $<10$ & 30 & $<5$ & -- & 40 & .- & -- \\
\hline $5 \mathrm{~S} / 3 \mathrm{~W}-9 \mathrm{H} 2$ & $<10$ & .- & $<10$ & 5,800 & 6.0 & -- & 170 & -- & .- \\
\hline 5S/3W-9Q1 & - & -- & -- & $<20$ & -- & -- & - & -- & -- \\
\hline 5S/3W-11M1 & -- & -- & -- & -- & -- & -- & -- & -- & -- \\
\hline \multirow[t]{18}{*}{$5 \mathrm{~S} / 3 \mathrm{~W}-11 \mathrm{M} 2$} & -- & -- & -- & - & -- & -- & -- & -- & -- \\
\hline & -- & -- & -- & -- & -- & -- & -- & -- & -- \\
\hline & -- & -- & -- & -- & -- & -- & -- & -- & -- \\
\hline & -- & -- & -- & -- & -- & -- & -- & - & -- \\
\hline & -- & -- & - & -- & -- & -- & - & - & -- \\
\hline & -- & -- & -- & -- & -- & -- & -- & -- & -- \\
\hline & -- & -- & -- & -- & -- & -- & -- & -- & -- \\
\hline & -- & -- & -- & -- & -- & -- & -- & -- & -- \\
\hline & -- & -- & -- & -- & -- & -- & -- & - & -- \\
\hline & -- & -- & -- & -- & -- & - & -- & -- & -- \\
\hline & -- & -- & -- & -- & -- & - & -- & - & -- \\
\hline & -- & -- & -- & -- & -- & - & -- & - & -- \\
\hline & -- & -- & - & -- & -- & - & -- & -- & -- \\
\hline & -- & -- & -- & - & -- & -- & -- & - & -- \\
\hline & -- & -- & - & - & -- & -- & -- & -- & -- \\
\hline & -- & -- & -- & & -- & -- & -- & -- & -- \\
\hline & -- & - & -- & -- & - & -- & -- & -- & -- \\
\hline & -- & -- & -- & $<20$ & -- & -- & -- & - & -- \\
\hline \multirow[t]{3}{*}{$5 S / 3 W-13 A 1$} & -- & -- & -- & - & -- & -- & -- & -- & -- \\
\hline & - & -- & -- & -- & -- & -- & -- & -- & -- \\
\hline & $<5$ & $<3$ & $<10$ & $<3$ & $<10$ & 9 & 7 & $<10$ & $<10$ \\
\hline \multirow[t]{2}{*}{$5 \mathrm{~S} / 3 \mathrm{~W}-13 \mathrm{H} 1$} & $<5$ & $<3$ & $<10$ & 5 & $<10$ & 20 & 6 & $<10$ & $<10$ \\
\hline & $<5$ & $<3$ & $<10$ & 20 & $<10$ & 20 & 2 & $<10$ & $<10$ \\
\hline \multirow[t]{3}{*}{$5 \mathrm{~S} / 3 \mathrm{~W}-14 \mathrm{P} 1$} & -- & -- & -- & - & -- & -- & -- & -- & - \\
\hline & -- & -- & -- & -- & -- & -- & -- & -- & -- \\
\hline & -- & -- & -- & - & -- & -- & -- & .- & .- \\
\hline $5 \mathrm{~S} / 3 \mathrm{~W}-15 \mathrm{H} 1$ & $<5$ & $<3$ & $<10$ & $<3$ & $<10$ & 10 & 10 & $<10$ & $<10$ \\
\hline $5 \mathrm{~S} / 3 \mathrm{~W}-16 \mathrm{D} 1$ & $<5$ & $<3$ & $<10$ & 4 & $<10$ & 20 & 1 & $<10$ & $<10$ \\
\hline $5 \mathrm{~S} / 3 \mathrm{~W}-16 \mathrm{~F} 1$ & $<5$ & $<3$ & $<10$ & $<3$ & $<10$ & 7 & $<1$ & $<10$ & $<10$ \\
\hline \multirow[t]{6}{*}{$5 S / 3 W-16 P 1$} & -- & -- & -- & -- & -- & -- & - & -- & -- \\
\hline & -- & -- & -- & -- & -- & -- & -- & -- & -- \\
\hline & - & -- & -- & -- & -- & -- & -. & - & - \\
\hline & -- & -- & -- & -- & -- & -- & -- & -. & -- \\
\hline & -- & -- & -- & -- & -- & - & -- & - & -- \\
\hline & -- & -- & _- & .- & - & .. & .- & -- & -- \\
\hline \multirow[t]{3}{*}{$5 \mathrm{~S} / 3 \mathrm{~W}-16 \mathrm{P} 2$} & -- & -- & -- & -- & -- & -- & -- & -- & -- \\
\hline & -- & -- & -- & -- & -- & -- & -- & -- & -- \\
\hline & - & -- & -- & $<20$ & -- & -- & -- & -- & -- \\
\hline 5S/3W-17R1 & $<10$ & - & $<10$ & 70 & $<5$ & -- & $<10$ & -- & -- \\
\hline $5 \mathrm{~S} / 3 \mathrm{~W}-17 \mathrm{R} 2$ & -- & -- & -- & -- & -- & - & - & - & -- \\
\hline \multirow[t]{2}{*}{$5 S / 3 W-21 C 1$} & .- & -- & -- & -- & - & -- & -- & -- & -- \\
\hline & -- & -- & -- & - & -- & _- & -- & -- & -- \\
\hline 5S/3W-21D1 & - & -- & -- & -- & -- & -- & -- & -- & -- \\
\hline
\end{tabular}


Table 2. Historical water-quality data for selected wells in the Winchester, Menifee, and south Perris ground-water subbasins, California-Continued

\begin{tabular}{|c|c|c|c|c|c|c|}
\hline Well No. & $\begin{array}{c}\text { Selenium, } \\
\text { dissolved } \\
(\mu \mathrm{g} / \mathrm{L})\end{array}$ & $\begin{array}{c}\text { Silver, } \\
\text { dissolved } \\
(\mu g / L)\end{array}$ & $\begin{array}{c}\text { Strontium, } \\
\text { dissolved } \\
(\mu g / L)\end{array}$ & $\begin{array}{c}\text { Vanadium, } \\
\text { dissolved } \\
(\mu \mathrm{g} / \mathrm{L})\end{array}$ & $\begin{array}{c}\text { Zinc, } \\
\text { dissolved } \\
(\mu \mathrm{g} / \mathrm{L})\end{array}$ & Source of data \\
\hline 5S/2W-31R1 & - & - & - & -- & - & EMWD \\
\hline \multirow[t]{2}{*}{$5 S / 2 W-33 E 1$} & - & - & -- & - & - & EMWD \\
\hline & -- & -- & - & -- & -- & EMWD \\
\hline $5 S / 3 W-2 Q 1$ & $<1$ & $<1.0$ & 170 & 50 & $<3$ & USGS \\
\hline $5 S / 3 W-3 C 1$ & $<5$ & $<10$ & -- & -. & 290 & Babcock \\
\hline $5 S / 3 W-3 L 1$ & $<5$ & $<10$ & -- & - & 260 & Babcock \\
\hline $5 S / 3 W-3 N 1$ & $<5$ & $<10$ & -- & -- & 260 & Babcock \\
\hline $5 S / 3 W-3 Q 2$ & -- & - & -- & -- & - & DWR \\
\hline $5 S / 3 W-3 R 2$ & - & - & -- & -- & -- & EMWD \\
\hline $5 S / 3 W-4 A 1$ & 6.0 & $<10$ & -- & $\ldots$ & 40 & Babcock \\
\hline $5 S / 3 W-4 M 1$ & $<10$ & $<10$ & -- & -- & 860 & Babcock \\
\hline 5S/3W-9E1 & $<5$ & $<10$ & - & - & 570 & Babcock \\
\hline $5 \mathrm{~S} / 3 \mathrm{~W}-9 \mathrm{H} 2$ & $<5$ & $<10$ & -- & - & 1,100 & Babcock \\
\hline $5 S / 3 W-9 Q 1$ & - & - & - & - & - & WA \\
\hline $5 S / 3 W-11 M 1$ & - & - & -- & - & - & RCFCD \\
\hline \multirow[t]{18}{*}{$5 S / 3 W-11 M 2$} & -- & -- & - & - & -- & RCFCD \\
\hline & - & - & -- & - & - & RCFCD * \\
\hline & - & -- & - & - & - & RCFCD * \\
\hline & - & -- & -- & - & - & RCFCD * \\
\hline & - & - & - & -- & -- & RCFCD \\
\hline & - & - & -- & - & -- & RCFCD \\
\hline & -- & - & -- & - & - & RCFCD * \\
\hline & - & - & - & - & - & RCFCD \\
\hline & - & -- & - & - & - & RCFCD \\
\hline & - & - & - & - & - & RCFCD \\
\hline & - & - & -- & -- & $\ldots$ & RCFCD \\
\hline & - & - & -- & -- & -- & RCFCD \\
\hline & - & - & - & - & - & RCFCD \\
\hline & - & - & - & - & - & RCFCD * \\
\hline & -- & - & - & - & - & EMWD \\
\hline & - & - & - & - & - & Babcock \\
\hline & - & - & -- & - & - & EMWD \\
\hline & -- & - & -- & -- & - & WA \\
\hline \multirow[t]{3}{*}{$5 S / 3 W-13 A 1$} & - & -- & - & - & -- & EMWD \\
\hline & - & -- & - & - & - & EMWD \\
\hline & 2.0 & $<1.0$ & 370 & 30 & 4 & USGS \\
\hline \multirow[t]{2}{*}{$5 S / 3 W-13 H 1$} & 2.0 & $<1.0$ & 480 & 24 & 7 & USGS \\
\hline & 2.0 & $<1.0$ & 430 & 26 & $<3$ & USGS \\
\hline \multirow[t]{3}{*}{$5 \mathrm{~S} / 3 \mathrm{~W}-14 \mathrm{P} 1$} & - & - & - & - & -- & DWR \\
\hline & - & - & - & - & - & EMWD \\
\hline & - & - & - & $\ldots$ & - & Babcock \\
\hline $5 \mathrm{~S} / 3 \mathrm{~W}-15 \mathrm{H} 1$ & 1.0 & $<1.0$ & 740 & 19 & 270 & USGS \\
\hline 5S/3W-16D1 & 5.0 & $<1.0$ & 1,400 & 29 & 100 & USGS \\
\hline $5 S / 3 W-16 F 1$ & 2.0 & $<1.0$ & 720 & 22 & 380 & USGS \\
\hline \multirow[t]{6}{*}{$5 S / 3 W-16 P_{1}$} & - & - & - & - & - & RCFCD \\
\hline & - & -- & - & - & -- & RCFCD \\
\hline & - & -- & -- & - & - & RCFCD \\
\hline & - & - & - & - & -- & RCFCD \\
\hline & - & - & - & -- & - & RCFCD \\
\hline & - & - & - & -- & - & RCFCD \\
\hline \multirow[t]{3}{*}{$5 S / 3 W-16 P 2$} & - & - & - & -- & - & EMWD \\
\hline & - & -- & - & -- & - & EMWD \\
\hline & -- & - & - & - & -- & WA \\
\hline 5S/3W-17R1 & 7.0 & $<10$ & - & - & 40 & Babcock \\
\hline 5S/3W-17R2 & - & - & - & -- & -- & Babcock \\
\hline \multirow[t]{2}{*}{$5 S / 3 W-21 C 1$} & - & - & - & -- & -- & EMWD \\
\hline & - & - & - & -- & - & EMWD \\
\hline 5S/3W-21D1 & - & -- & - & - & - & RCFCD \\
\hline
\end{tabular}


Table 2. Historical water-quality data for selected wells in the Winchester, Menifee, and south Perris ground-water subbasins, California--Continued

\begin{tabular}{|c|c|c|c|c|c|c|c|c|}
\hline \multirow[t]{2}{*}{ Well No. } & \multirow[t]{2}{*}{ Date } & \multirow{2}{*}{$\begin{array}{l}\text { Water level } \\
\text { (ft blw LSD) }\end{array}$} & \multirow{2}{*}{$\begin{array}{c}\text { Well depth } \\
\text { (ft) }\end{array}$} & \multirow{2}{*}{$\begin{array}{c}\text { Altitude } \\
\text { of LSD (ft) }\end{array}$} & \multicolumn{2}{|c|}{ Specific conductance $(\mu \mathrm{S} / \mathrm{cm})$} & \multirow{2}{*}{$\begin{array}{c}\mathrm{pH} \text { (lab) } \\
\text { (Std. units) }\end{array}$} & \multirow{2}{*}{$\begin{array}{c}\text { Water } \\
\text { temperature } \\
\left({ }^{\circ} \mathrm{C}\right) \\
\end{array}$} \\
\hline & & & & & Field & Lab & & \\
\hline & $4 / 23 / 63$ & -- & & & -- & 716 & 7.6 & 25.5 \\
\hline & $9 / 27 / 63$ & -- & & & -- & 1,250 & 7.6 & 28.0 \\
\hline & $4 / 17 / 64$ & - & & & - & 726 & 7.7 & 16.5 \\
\hline & $8 / 13 / 64$ & -- & & & - & 680 & 7.7 & 22.0 \\
\hline & $3 / 30 / 65$ & - & & & -- & 1,180 & 8.2 & 20.0 \\
\hline & $9 / 24 / 65$ & - & & & - & 2,030 & 7.6 & 25.5 \\
\hline & $3 / 23 / 66$ & -- & & & - & 1,050 & 7.7 & 22.0 \\
\hline & $9 / 15 / 66$ & -- & & & -- & 1,740 & 7.7 & 28.0 \\
\hline & $5 / 11 / 67$ & -- & & & - & 1,080 & 7.8 & 19.0 \\
\hline & 9/29/67 & -- & & & - & 3,710 & 7.6 & 22.0 \\
\hline & $4 / 23 / 68$ & -- & & & -- & 2,950 & 7.6 & 24.5 \\
\hline & $4 / 22 / 69$ & -- & & & - & 1,500 & 7.6 & 23.5 \\
\hline & $10 / 23 / 69$ & - & & & -- & 3,940 & 7.5 & 23.5 \\
\hline & $4 / 28 / 70$ & -- & & & - & 1,990 & 8.0 & 22.0 \\
\hline & $11 / 18 / 70$ & -- & & & -- & 1,990 & 8.1 & 24.5 \\
\hline & $4 / 30 / 71$ & -- & & & -- & 1,900 & 8.2 & 24.5 \\
\hline \multirow[t]{17}{*}{$5 S / 3 W-21 D 2$} & $3 / 25 / 60$ & -- & 452 & - & -- & 2,110 & 7.5 & 24.5 \\
\hline & $9 / 14 / 60$ & - & & & -- & 2,170 & 7.4 & 24.5 \\
\hline & $4 / 13 / 61$ & - & & & -- & 2,380 & 8.0 & 26.0 \\
\hline & $9 / 28 / 61$ & - & & & - & 2,380 & 8.1 & 24.5 \\
\hline & $9 / 24 / 62$ & -- & & & - & 2,440 & 7.5 & 26.0 \\
\hline & $4 / 17 / 64$ & - & & & - & 2,620 & 7.5 & 26.0 \\
\hline & $8 / 13 / 64$ & -- & & & - & 2,500 & 8.0 & 24.5 \\
\hline & $3 / 23 / 66$ & - & & & -- & 2,690 & 7.7 & 24.5 \\
\hline & $5 / 11 / 67$ & - & & & - & 2,540 & 7.7 & 26.0 \\
\hline & $10 / 15 / 68$ & - & & & - & 2,090 & 7.7 & 25.5 \\
\hline & $4 / 30 / 71$ & -- & & & -- & 2,770 & 7.8 & 23.5 \\
\hline & $4 / 27 / 73$ & -- & & & -- & 1,790 & 7.7 & 24.5 \\
\hline & $9 / 26 / 73$ & -- & & & -- & $.3,030$ & 7.6 & 26.0 \\
\hline & $5 / 3 / 74$ & - & & & - & 3,640 & 7.4 & 26.0 \\
\hline & $9 / 24 / 74$ & - & & & -- & 2,980 & 8.0 & 21.0 \\
\hline & $11 / 7 / 75$ & -- & & & -- & 3,900 & 7.3 & 24.0 \\
\hline & $4 / 20 / 77$ & -- & & & -- & -- & -- & - \\
\hline \multirow[t]{3}{*}{$5 \mathrm{~S} / 3 \mathrm{~W}-24 \mathrm{C} 1$} & $11 / 18 / 93$ & - & 505 & 1,480 & 921 & 927 & 7.4 & 21.0 \\
\hline & $1 / 25 / 94$ & -- & & & 910 & 913 & 7.3 & 21.0 \\
\hline & $6 / 24 / 94$ & -- & & & 832 & 827 & 7.0 & 30.5 \\
\hline $5 S / 3 W-24 F 2$ & $6 / 15 / 94$ & -- & 691 & 1,475 & 695 & 698 & 7.7 & 23.5 \\
\hline $5 S / 3 W-24 F 3$ & $6 / 15 / 94$ & - & 403.8 & 1,475 & 3,790 & 3,810 & 6.4 & 23.5 \\
\hline $5 \mathrm{~S} / 3 \mathrm{~W}-24 \mathrm{~F} 4$ & $6 / 15 / 94$ & - & 155.1 & 1,475 & 1,880 & 1,890 & 6.8 & 22.0 \\
\hline \multirow[t]{7}{*}{$5 \mathrm{~S} / 3 \mathrm{~W}-28 \mathrm{M} 1$} & $9 / 11 / 90$ & - & 430 & 1,420 & - & 238 & 6.9 & - \\
\hline & $1 / 31 / 91$ & 72.89 & & & 1,690 & 1,690 & 7.8 & 22.5 \\
\hline & $6 / 13 / 91$ & 69.08 & & & 1,730 & 1,750 & 7.5 & 23.0 \\
\hline & $8 / 30 / 91$ & 69.13 & & & 1,730 & 1,740 & 7.3 & 23.0 \\
\hline & $1 / 30 / 92$ & 67.83 & & & 1,710 & 1,600 & 7.5 & 23.0 \\
\hline & $8 / 19 / 92$ & 62.79 & & & 1,740 & 1,750 & 8.0 & 24.0 \\
\hline & $12 / 10 / 93$ & - & & & 1,760 & 1,770 & 7.4 & 22.5 \\
\hline \multirow[t]{6}{*}{$5 \mathrm{~S} / 3 \mathrm{~W}-28 \mathrm{M} 2$} & $1 / 31 / 91$ & 72.52 & 370 & 1,420 & 2,380 & 2,430 & 7.3 & 21.5 \\
\hline & $6 / 13 / 91$ & 68.84 & & & 2,420 & 2,300 & 7.3 & 22.5 \\
\hline & $8 / 30 / 91$ & 68.73 & & & 2,440 & 2,460 & 7.2 & 23.0 \\
\hline & $1 / 31 / 92$ & 67.61 & & & 2,410 & 2,400 & 7.3 & 22.5 \\
\hline & $8 / 19 / 92$ & 62.62 & & & 2,420 & 2,440 & 7.8 & 24.0 \\
\hline & $12 / 10 / 93$ & - & & & 2,420 & 2,440 & 7.1 & 21.0 \\
\hline \multirow[t]{6}{*}{$5 \mathrm{~S} / 3 \mathrm{~W}-28 \mathrm{M} 3$} & 1/31/91 & 62.11 & 242 & 1,420 & 5,380 & 5,520 & 6.6 & 21.5 \\
\hline & $6 / 14 / 91$ & 60.11 & & & 5,450 & 5,550 & 6.6 & 22.0 \\
\hline & $8 / 30 / 91$ & 59.18 & & & 5,450 & 5,540 & 6.5 & 22.5 \\
\hline & $1 / 31 / 92$ & 59.3 & & & 5,410 & 5,550 & 6.8 & 21.5 \\
\hline & $8 / 28 / 92$ & 55.64 & & & 5,460 & 5,270 & 6.9 & 22.0 \\
\hline & $12 / 10 / 93$ & -- & & & 5,530 & 5,550 & 6.4 & 21.0 \\
\hline
\end{tabular}


Table 2. Historical water-quality data for selected wells in the Winchester, Menifee, and south Perris ground-water subbasins, California--Continued

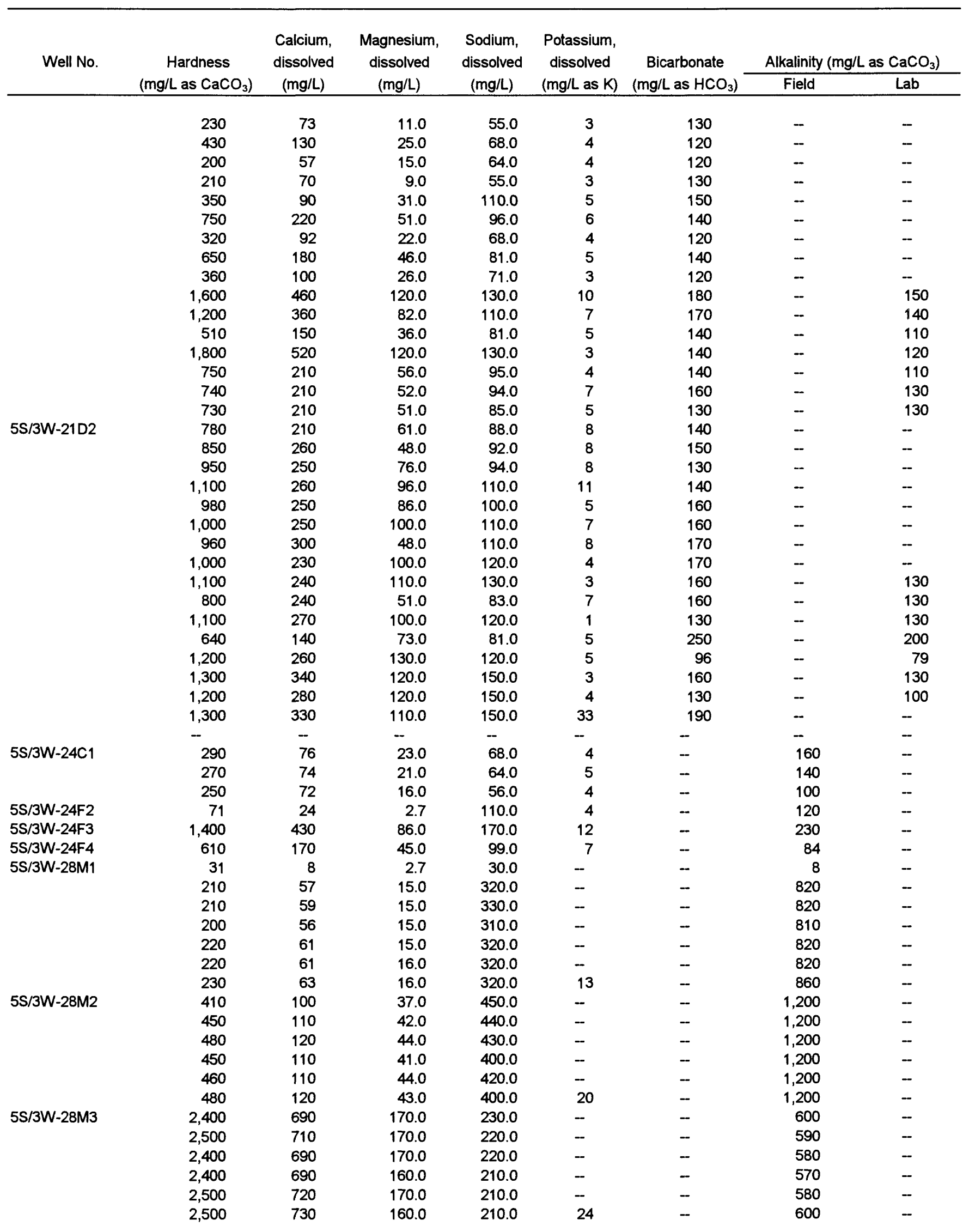


Table 2. Historical water-quality data for selected wells in the Winchester, Menifee, and south Perris ground-water subbasins, California-Continued

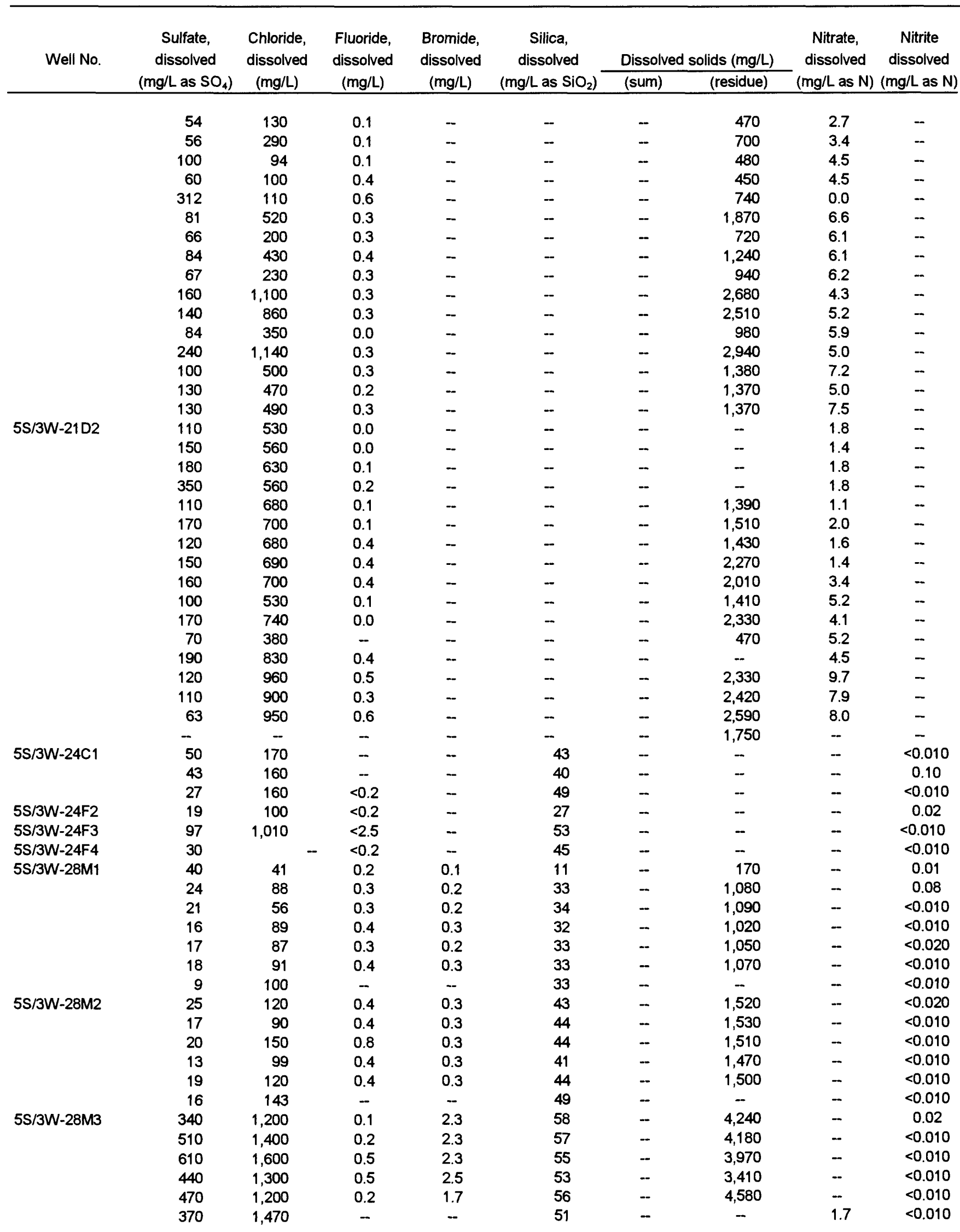


Table 2. Historical water-quality data for selected wells in the Winchester, Menifee, and south Perris ground-water subbasins, California--Continued

\begin{tabular}{|c|c|c|c|c|c|c|c|c|c|}
\hline Well No. & $\begin{array}{c}\mathrm{NO}_{3}+\mathrm{NO}_{2} \\
\text { dissolved } \\
(\mathrm{mg} / \mathrm{L} \text { as } \mathrm{N})\end{array}$ & $\begin{array}{c}\text { Nitrogen } \\
\text { ammonia, } \\
\text { dissolved } \\
\text { (mg/L as } \mathrm{N} \text { ) }\end{array}$ & $\begin{array}{c}\text { Phosphorus, } \\
\text { dissolved } \\
(\mathrm{mg} / \mathrm{L})\end{array}$ & $\begin{array}{c}\text { Phosphorus } \\
\text { ortho, } \\
\text { dissolved } \\
\text { (mg/L as P) }\end{array}$ & $\begin{array}{c}\text { Arsenic, } \\
\text { dissolved } \\
(\mu \mathrm{g} / \mathrm{L})\end{array}$ & $\begin{array}{c}\text { Barium, } \\
\text { dissolved } \\
(\mu \mathrm{g} / \mathrm{L})\end{array}$ & $\begin{array}{c}\text { Beryllium, } \\
\text { dissolved } \\
(\mu \mathrm{g} / \mathrm{L}) \\
\end{array}$ & $\begin{array}{c}\text { Boron, } \\
\text { dissolved } \\
(\mu \mathrm{g} / \mathrm{L})\end{array}$ & $\begin{array}{c}\text { Cadmium, } \\
\text { dissolved } \\
(\mu \mathrm{g} / \mathrm{L})\end{array}$ \\
\hline & -- & - & -- & - & -- & - & -- & 100 & - \\
\hline & -- & -- & -- & -- & - & -- & -- & 100 & - \\
\hline & -- & - & - & -- & - & -- & - & 100 & -- \\
\hline & - & - & - & -- & -- & -- & -- & 400 & -- \\
\hline & - & -- & - & - & -- & -- & - & 190 & - \\
\hline & -- & -- & -- & - & -- & -- & -- & 50 & - \\
\hline & -- & - & - & -- & - & -- & -- & 30 & -- \\
\hline & -- & -- & - & -- & -- & -- & -- & 60 & - \\
\hline & -- & -. & -- & -- & - & - & -- & 40 & - \\
\hline & -- & - & -- & -- & -- & -- & -- & 50 & - \\
\hline & - & -- & -- & - & -- & - & -- & 50 & -- \\
\hline & -- & - & -- & -- & -- & - & -- & 30 & - \\
\hline & - & -- & -- & -- & -- & -- & -- & 30 & - \\
\hline & - & -- & -- & - & -- & - & -- & 0 & - \\
\hline & -- & -- & -- & -- & -- & - & -- & 0 & -- \\
\hline & - & - & - & -- & - & - & - & 60 & - \\
\hline \multirow[t]{17}{*}{$5 S / 3 W-21 D 2$} & -- & - & -- & -- & -- & - & - & 300 & -- \\
\hline & -- & - & -- & - & - & -- & - & 100 & - \\
\hline & -- & - & -- & - & -- & - & -- & 300 & -- \\
\hline & -- & -- & - & -- & -- & -- & - & 400 & -- \\
\hline & -- & -- & -- & -- & -- & -- & - & 100 & -- \\
\hline & -- & - & - & - & - & - & -- & 300 & - \\
\hline & -- & -- & - & -- & - & - & - & 300 & - \\
\hline & -- & - & - & -- & -- & - & - & 210 & - \\
\hline & - & - & -- & -- & -- & -- & - & 290 & - \\
\hline & - & - & - & -- & -- & -- & - & 70 & -- \\
\hline & - & - & - & - & - & -- & - & 170 & -- \\
\hline & - & - & -- & -- & - & -- & - & -- & -- \\
\hline & - & -- & - & - & -- & -- & - & 200 & -- \\
\hline & - & - & -- & - & - & - & - & 20 & -- \\
\hline & - & - & -- & - & - & - & -- & 20 & - \\
\hline & - & - & -- & - & - & - & - & 300 & -- \\
\hline & - & -- & -- & - & - & - & - & -- & -- \\
\hline \multirow[t]{3}{*}{$5 S / 3 W-24 C 1$} & 6.6 & 0.03 & -- & 0.03 & 2.0 & 200 & $<0.5$ & 40 & -- \\
\hline & 6.6 & 0.06 & -- & 0.03 & 2.0 & 190 & $<0.5$ & 50 & - \\
\hline & 5.0 & 0.02 & -- & 0.05 & 2.0 & 170 & $<0.5$ & 40 & - \\
\hline $5 S / 3 W-24 F 2$ & 6.1 & 0.02 & - & 0.80 & 2.0 & 23 & $<0.5$ & 90 & -- \\
\hline $5 S / 3 W-24 F 3$ & 15 & 0.06 & - & 1.0 & 6.0 & 470 & $<2$ & 80 & -- \\
\hline $5 S / 3 W-24 F 4$ & 14 & 0.03 & - & 1.0 & 4.0 & 410 & $<0.5$ & 50 & - \\
\hline \multirow[t]{7}{*}{$5 \mathrm{~S} / 3 \mathrm{~W}-28 \mathrm{M} 1$} & 0.22 & 0.02 & 0.03 & 0.01 & - & 9.0 & $<0.5$ & 30 & - \\
\hline & $<0.10$ & 0.02 & 0.45 & 0.44 & - & 300 & $<0.5$ & 190 & - \\
\hline & $<0.05$ & 0.02 & 0.50 & 0.48 & -- & 300 & $<0.5$ & 200 & -- \\
\hline & $<0.05$ & 0.03 & 0.46 & 0.41 & -- & 330 & $<0.5$ & 180 & - \\
\hline & $<0.05$ & 0.03 & 0.28 & 0.27 & - & 350 & $<0.5$ & 200 & - \\
\hline & $<0.05$ & 0.03 & 0.28 & 0.28 & - & 350 & $<0.5$ & 190 & -- \\
\hline & $<0.05$ & $<0.01$ & -- & 0.36 & 3.0 & 390 & $<0.5$ & 180 & -- \\
\hline \multirow[t]{6}{*}{$5 \mathrm{~S} / 3 \mathrm{~W}-28 \mathrm{M} 2$} & $<0.10$ & 0.03 & 1.7 & 1.5 & -- & 430 & $<2$ & 200 & -- \\
\hline & $<0.05$ & 0.02 & 1.6 & 1.4 & - & 460 & $<2$ & 220 & - \\
\hline & $<0.05$ & 0.03 & 1.8 & 1.7 & - & 470 & $<2$ & 210 & - \\
\hline & $<0.05$ & 0.03 & 1.8 & 1.7 & - & 450 & $<2$ & 230 & - \\
\hline & $<0.05$ & 0.04 & 2.1 & 2.1 & - & 490 & $<2$ & 230 & - \\
\hline & 0.08 & $<0.01$ & -- & 2.9 & 14 & 520 & $<2$ & 220 & - \\
\hline \multirow[t]{6}{*}{$5 S / 3 W-28 M 3$} & 1.6 & 0.06 & 0.84 & 0.61 & -- & 96 & $<2$ & 200 & -- \\
\hline & 1.7 & 0.04 & 0.91 & 0.72 & -- & 91 & $<2$ & 230 & - \\
\hline & 1.7 & 0.03 & 0.97 & 0.82 & - & 91 & $<2$ & 210 & - \\
\hline & 1.6 & 0.03 & 0.98 & 0.86 & -- & 89 & $<2$ & 230 & -- \\
\hline & 1.7 & 0.16 & 1.3 & 1.2 & - & 90 & $<2$ & 230 & -- \\
\hline & 1.7 & 0.07 & - & 1.6 & 2.0 & 95 & $<2$ & 240 & -- \\
\hline
\end{tabular}


Table 2. Historical water-quality data for selected wells in the Winchester, Menifee, and south Perris ground-water subbasins, California-Continued

\begin{tabular}{|c|c|c|c|c|c|c|c|c|c|}
\hline Well No. & $\begin{array}{c}\text { Chromium, } \\
\text { dissolved } \\
(\mu \mathrm{g} / \mathrm{L}) \\
\end{array}$ & $\begin{array}{c}\text { Cobalt, } \\
\text { dissolved } \\
(\mu \mathrm{g} / \mathrm{L})\end{array}$ & $\begin{array}{c}\text { Copper, } \\
\text { dissolved } \\
(\mu g / L)\end{array}$ & $\begin{array}{c}\text { Iron, } \\
\text { dissolved } \\
(\mu g / L) \\
\end{array}$ & $\begin{array}{c}\text { Lead, } \\
\text { dissolved } \\
(\mu g / L)\end{array}$ & $\begin{array}{c}\text { Lithium, } \\
\text { dissolved } \\
(\mu g / L)\end{array}$ & $\begin{array}{c}\text { Manganese, } \\
\text { dissolved } \\
(\mu \mathrm{g} / \mathrm{L}) \\
\end{array}$ & $\begin{array}{c}\text { Molybdenum, } \\
\text { dissolved } \\
(\mu \mathrm{g} / \mathrm{L})\end{array}$ & $\begin{array}{c}\text { Nickel, } \\
\text { dissolved } \\
(\mu g / L)\end{array}$ \\
\hline & - & - & - & -- & -- & - & - & - & - \\
\hline & - & -- & -- & -- & - & - & -- & -- & - \\
\hline & -- & - & - & - & - & - & - & -- & - \\
\hline & - & -- & -- & -- & -- & -- & - & -- & - \\
\hline & -- & -- & -- & - & -- & - & -- & -- & - \\
\hline & - & -- & -- & - & -- & - & -- & -- & - \\
\hline & -- & - & - & - & - & - & -- & - & - \\
\hline & - & -- & - & - & -- & - & - & - & -- \\
\hline & - & -- & - & -- & -- & - & - & -- & -- \\
\hline & - & - & - & - & -- & - & - & - & - \\
\hline & - & - & - & -- & -- & - & -- & - & -- \\
\hline & - & -- & - & - & - & -- & - & - & -- \\
\hline & -- & - & -- & - & - & - & -- & -- & - \\
\hline & -- & -- & -- & - & -- & - & - & -- & - \\
\hline & - & - & -- & - & -- & -- & -- & -- & - \\
\hline & - & - & -- & - & - & - & -- & - & - \\
\hline \multirow[t]{17}{*}{$5 S / 3 W-21 D 2$} & - & - & - & - & -- & -- & - & - & - \\
\hline & - & - & -- & - & -- & - & -- & -- & - \\
\hline & - & - & -- & -- & - & - & - & -- & - \\
\hline & -- & - & -- & -- & - & - & -- & -- & - \\
\hline & -- & - & - & -- & -- & - & - & -- & -- \\
\hline & - & -- & - & -- & - & - & - & - & - \\
\hline & -- & -- & -- & -- & -- & - & - & -- & - \\
\hline & -- & -- & - & -- & -- & - & - & -- & - \\
\hline & - & -- & - & -- & -- & - & - & - & - \\
\hline & -- & -- & - & - & - & - & - & - & -- \\
\hline & -- & - & - & -- & - & - & - & - & -- \\
\hline & - & - & - & -- & -- & - & - & -- & - \\
\hline & - & -- & - & -- & -- & -- & -- & - & -- \\
\hline & -- & -- & -- & - & - & - & - & -- & - \\
\hline & - & -- & - & -- & - & -- & -- & - & -- \\
\hline & -- & -- & - & -- & - & -- & -- & - & -- \\
\hline & -- & - & -- & -- & - & -- & -- & - & -- \\
\hline \multirow[t]{3}{*}{$5 S / 3 W-24 C 1$} & $<5$ & $<3$ & $<10$ & 20 & $<10$ & 5 & 9 & $<10$ & $<10$ \\
\hline & $<5$ & $<3$ & $<10$ & 20 & $<10$ & 5 & 27 & $<10$ & $<10$ \\
\hline & $<5$ & $<3$ & $<10$ & 8 & 10 & 10 & 1 & $<10$ & $<10$ \\
\hline $5 S / 3 W-24 F 2$ & $<5$ & $<3$ & $<10$ & 0 & $<10$ & 7 & 83 & 20 & $<10$ \\
\hline $5 S / 3 W-24 F 3$ & $<20$ & $<9$ & $<30$ & $<9$ & $<30$ & 40 & 280 & $<30$ & $<30$ \\
\hline $5 S / 3 W-24 F 4$ & $<5$ & $<3$ & $<10$ & 20 & $<10$ & 10 & 53 & $<10$ & $<10$ \\
\hline \multirow[t]{7}{*}{$5 S / 3 W-28 M 1$} & $<5$ & $<3$ & $<10$ & 320 & $<10$ & $<4$ & 28 & $<10$ & $<10$ \\
\hline & $<5$ & $<3$ & $<10$ & 20 & $<10$ & 30 & 180 & 10 & $<10$ \\
\hline & $<5$ & $<3$ & $<10$ & 20 & $<10$ & 30 & 170 & $<10$ & $<10$ \\
\hline & $<5$ & $<3$ & $<10$ & 20 & $<10$ & 20 & 170 & 20 & $<10$ \\
\hline & $<5$ & $<3$ & $<10$ & 20 & $<10$ & 30 & 150 & 10 & $<10$ \\
\hline & $<5$ & $<3$ & $<10$ & 10 & $<10$ & 30 & 150 & 10 & $<10$ \\
\hline & $<5$ & $<3$ & $<10$ & 30 & $<10$ & 30 & 160 & $<10$ & $<10$ \\
\hline \multirow[t]{6}{*}{$5 S / 3 W-28 M 2$} & $<20$ & $<9$ & $<30$ & 100 & $<30$ & 30 & 120 & $<30$ & $<30$ \\
\hline & $<20$ & $<9$ & $<30$ & 120 & $<30$ & 40 & 120 & $<30$ & $<30$ \\
\hline & $<20$ & $<9$ & $<30$ & 110 & $<30$ & 40 & 140 & $<30$ & $<30$ \\
\hline & $<20$ & $<9$ & $<30$ & 90 & $<30$ & 40 & 130 & $<30$ & $<30$ \\
\hline & $<20$ & $<9$ & $<30$ & 80 & $<30$ & 40 & 170 & $<30$ & $<30$ \\
\hline & $<20$ & $<9$ & $<30$ & 40 & $<30$ & 30 & 250 & $<30$ & $<30$ \\
\hline \multirow[t]{6}{*}{$5 S / 3 W-28 M 3$} & $<20$ & $<9$ & $<30$ & 20 & $<30$ & 80 & 370 & $<30$ & $<30$ \\
\hline & $<20$ & $<9$ & $<30$ & 20 & $<30$ & 80 & 390 & $<30$ & $<30$ \\
\hline & $<20$ & $<9$ & $<30$ & 10 & $<30$ & 90 & 380 & $<30$ & $<30$ \\
\hline & $<20$ & $<9$ & $<30$ & $<9$ & $<30$ & 90 & 410 & $<30$ & $<30$ \\
\hline & $<20$ & $<9$ & $<30$ & $<9$ & $<30$ & 50 & 400 & $<30$ & $<30$ \\
\hline & 160 & $<9$ & $<30$ & 650 & $<30$ & 70 & 660 & $<30$ & $<30$ \\
\hline
\end{tabular}


Table 2. Historical water-quality data for selected wells in the Winchester, Menifee, and south Perris ground-water subbasins, California-Continued

\begin{tabular}{|c|c|c|c|c|c|c|}
\hline Well No. & $\begin{array}{c}\text { Selenium, } \\
\text { dissolved } \\
(\mu \mathrm{g} / \mathrm{L})\end{array}$ & $\begin{array}{c}\text { Silver, } \\
\text { dissolved } \\
(\mu \mathrm{g} / \mathrm{L})\end{array}$ & $\begin{array}{c}\text { Strontium, } \\
\text { dissolved } \\
(\mu \mathrm{g} / \mathrm{L})\end{array}$ & $\begin{array}{c}\text { Vanadium, } \\
\text { dissolved } \\
(\mu \mathrm{g} / \mathrm{L})\end{array}$ & $\begin{array}{c}\text { Zinc, } \\
\text { dissolved } \\
(\mu \mathrm{g} / \mathrm{L})\end{array}$ & Source of data \\
\hline & -- & - & - & -- & - & RCFCD \\
\hline & -- & - & - & -- & - & RCFCD \\
\hline & - & -- & -- & -- & - & RCFCD \\
\hline & -- & -- & -- & -- & -- & RCFCD \\
\hline & -- & - & - & -- & - & RCFCD \\
\hline & -- & - & - & - & -- & RCFCD \\
\hline & -- & - & - & - & -- & RCFCD \\
\hline & -- & - & - & - & - & RCFCD \\
\hline & -- & -- & - & -- & - & RCFCD \\
\hline & - & -- & - & -- & - & RCFCD \\
\hline & - & -- & - & - & - & RCFCD \\
\hline & - & - & - & -- & - & RCFCD \\
\hline & -- & -- & - & -- & - & RCFCD \\
\hline & -- & -- & - & - & -- & RCFCD \\
\hline & -- & - & - & - & - & RCFCD \\
\hline & -- & -- & -- & -- & -- & RCFCD \\
\hline \multirow[t]{17}{*}{$5 S / 3 W-21 D 2$} & -- & -- & -- & - & - & RCFCD \\
\hline & -- & -- & - & -- & -- & RCFCD \\
\hline & -- & -- & - & -- & - & RCFCD \\
\hline & - & - & - & -- & - & RCFCD \\
\hline & - & -- & -- & - & - & RCFCD \\
\hline & - & -- & - & - & -- & RCFCD \\
\hline & -- & - & - & -- & -- & RCFCD \\
\hline & -- & -- & - & - & -- & RCFCD \\
\hline & - & -- & - & - & -- & RCFCD \\
\hline & - & -- & - & - & - & RCFCD \\
\hline & -- & -- & - & -- & -- & RCFCD \\
\hline & - & -- & - & -- & - & RCFCD \\
\hline & - & -- & - & - & -- & RCFCD \\
\hline & -- & -- & - & -- & - & RCFCD \\
\hline & -- & - & - & - & -- & RCFCD \\
\hline & -- & - & - & - & -- & EMWD \\
\hline & -- & - & - & - & - & EMWD \\
\hline \multirow[t]{3}{*}{$5 S / 3 W-24 C 1$} & 2.0 & $<1.0$ & 390 & 20 & $<3$ & USGS \\
\hline & 2.0 & $<1.0$ & 370 & 20 & 9 & USGS \\
\hline & - & 2.0 & 320 & 19 & 9 & USGS \\
\hline $5 S / 3 W-24 F 2$ & -- & $<1.0$ & 240 & 10 & 10 & USGS \\
\hline $5 S / 3 W-24 F 3$ & - & $<3.0$ & 2,200 & 20 & $<9$ & USGS \\
\hline $5 S / 3 W-24 F 4$ & - & $<1.0$ & 1,200 & 22 & 8 & USGS \\
\hline \multirow[t]{7}{*}{$5 \mathrm{~S} / 3 \mathrm{~W}-28 \mathrm{M} 1$} & $<2$ & $<1.0$ & 40 & 21 & 7 & USGS \\
\hline & $<1$ & $<1.0$ & 1,400 & $<6$ & $<3$ & USGS \\
\hline & $<1$ & $<1.0$ & 1,400 & $<6$ & 5 & USGS \\
\hline & $<1$ & $<1.0$ & 1,500 & $<6$ & $<3$ & USGS \\
\hline & $<1$ & 1.0 & 1,500 & $<6$ & 3 & USGS \\
\hline & $<1$ & $<1.0$ & 1,600 & $<6$ & $<3$ & USGS \\
\hline & $<1$ & $<1.0$ & 1,700 & $<6$ & $<3$ & USGS \\
\hline \multirow[t]{6}{*}{$5 S / 3 W-28 M 2$} & $<1$ & $<3.0$ & 2,500 & $<18$ & $<9$ & USGS \\
\hline & $<1$ & $<3.0$ & 2,700 & $<18$ & $<9$ & USGS \\
\hline & $<1$ & $<3.0$ & 2,900 & $<18$ & $<9$ & USGS \\
\hline & $<1$ & $<3.0$ & 2,600 & $<18$ & 20 & USGS \\
\hline & $<1$ & $<3.0$ & 2,900 & $<18$ & $<9$ & USGS \\
\hline & $<1$ & $<3.0$ & 2,900 & $<18$ & 10 & USGS \\
\hline \multirow[t]{6}{*}{$5 S / 3 W-28 M 3$} & 7.0 & 5.0 & 7,900 & $<18$ & 10 & USGS \\
\hline & 10 & $<3.0$ & 7,900 & $<18$ & 10 & USGS \\
\hline & 8.0 & $<3.0$ & 7,700 & $<18$ & 20 & USGS \\
\hline & 8.0 & $<3.0$ & 7,700 & $<18$ & 30 & USGS \\
\hline & 9.0 & $<3.0$ & 8,100 & $<18$ & $<9$ & USGS \\
\hline & 8.0 & $<3.0$ & 7,600 & $<18$ & 40 & USGS \\
\hline
\end{tabular}


Table 2. Historical water-quality data for selected wells in the Winchester, Menifee, and south Perris ground-water subbasins, California--Continued

\begin{tabular}{|c|c|c|c|c|c|c|c|c|}
\hline \multirow[t]{2}{*}{ Well No. } & \multirow[t]{2}{*}{ Date } & \multirow{2}{*}{$\begin{array}{l}\text { Water level } \\
\text { (ft blw LSD) }\end{array}$} & \multirow{2}{*}{$\begin{array}{c}\text { Well depth } \\
\text { (ft) }\end{array}$} & \multirow{2}{*}{$\begin{array}{c}\text { Altitude } \\
\text { of LSD (ft) }\end{array}$} & \multicolumn{2}{|c|}{ Specific conductance $(\mu \mathrm{S} / \mathrm{cm})$} & \multirow{2}{*}{$\begin{array}{c}\text { pH (lab) } \\
\text { (Std. units) }\end{array}$} & \multirow{2}{*}{$\begin{array}{c}\text { Water } \\
\text { temperature } \\
\left({ }^{\circ} \mathrm{C}\right)\end{array}$} \\
\hline & & & & & Field & Lab & & \\
\hline \multirow[t]{6}{*}{$5 S / 3 W-28 M 4$} & $1 / 31 / 91$ & 62.4 & 160 & 1,420 & 4,300 & 4,380 & 6.7 & 20.5 \\
\hline & $6 / 14 / 91$ & 60.51 & & & 4,320 & 4,380 & 6.7 & 21.0 \\
\hline & $8 / 30 / 91$ & 59.38 & & & 4,320 & 4,370 & 7.1 & 21.0 \\
\hline & $1 / 30 / 92$ & 59.52 & & & 4,320 & 4,390 & 7.1 & 20.5 \\
\hline & $8 / 28 / 92$ & 55.84 & & & 4,300 & 4,330 & 7.0 & 21.0 \\
\hline & $12 / 10 / 93$ & -- & & & 4,240 & 4,290 & 7.1 & -- \\
\hline \multirow[t]{8}{*}{ 5S/3W-28M5LYS } & 2/25/91 & unsat. zone & 63 & 1,420 & 5,140 & 4,820 & 7.5 & 19.5 \\
\hline & $6 / 5 / 91$ & unsat. zone & & & 2,100 & 4,420 & 7.3 & 20.5 \\
\hline & $7 / 5 / 91$ & unsat. zone & & & -- & -- & -- & - \\
\hline & $8 / 12 / 91$ & unsat. zone & & & 4,490 & 4,380 & 7.6 & 21.5 \\
\hline & $2 / 11 / 92$ & unsat. zone & & & 4,500 & 4,490 & 7.6 & 18.5 \\
\hline & $5 / 5 / 92$ & unsat. zone & & & 4,540 & 4,540 & 7.6 & 21.0 \\
\hline & $8 / 7 / 92$ & unsat. zone & & & 4,480 & 4,430 & 7.7 & 21.0 \\
\hline & $9 / 8 / 92$ & unsat. zone & & & - & - & - & -- \\
\hline \multirow[t]{5}{*}{ 5S/3W-28M6LYS } & $8 / 1 / 91$ & unsat. zone & 45 & 1,420 & -- & -- & -- & -- \\
\hline & $2 / 11 / 92$ & unsat. zone & & & 9,330 & 9,250 & 7.3 & 18.0 \\
\hline & $5 / 5 / 92$ & unsat. zone & & & 9,110 & -- & - & 21.5 \\
\hline & $8 / 7 / 92$ & unsat. zone & & & 8,990 & 9,020 & 7.8 & -- \\
\hline & $9 / 8 / 92$ & unsat. zone & & & - & -- & -- & -- \\
\hline \multirow[t]{7}{*}{ 5S/3W-28M7LYS } & $2 / 25 / 91$ & unsat. zone & 35 & 1,420 & 9,360 & 8,890 & 7.3 & 20.0 \\
\hline & $6 / 5 / 91$ & unsat. zone & & & 6,670 & 9,250 & 7.1 & 21.0 \\
\hline & $10 / 22 / 91$ & unsat. zone & & & - & -- & - & - \\
\hline & $2 / 11 / 92$ & unsat. zone & & & 9.450 & 9,350 & 7.0 & 18.5 \\
\hline & $5 / 5 / 92$ & unsat. zone & & & 9,340 & -- & -- & 21.0 \\
\hline & $8 / 7 / 92$ & unsat. zone & & & 9,200 & 9,210 & 7.7 & - \\
\hline & $9 / 8 / 92$ & unsat. zone & & & -- & -- & -- & - \\
\hline \multirow[t]{3}{*}{ 5S/3W-28M8LYS } & $5 / 5 / 92$ & unsat. zone & 25 & 1,420 & 9,570 & 9,490 & 7.1 & 20.5 \\
\hline & $8 / 7 / 92$ & unsat. zone & & & 9,150 & 9,170 & 7.5 & -- \\
\hline & $9 / 8 / 92$ & unsat. zone & & & - & - & -- & -- \\
\hline \multirow[t]{7}{*}{ 5S/3W-28M9LYS } & $6 / 5 / 91$ & unsat. zone & 15 & 1,420 & 4,570 & 6,980 & 7.1 & 19.5 \\
\hline & $7 / 5 / 91$ & unsat. zone & & & - & -- & -- & - \\
\hline & $8 / 12 / 91$ & unsat. zone & & & 6,570 & 6,560 & 7.1 & 21.0 \\
\hline & $2 / 11 / 92$ & unsat. zone & & & 6,530 & 6,490 & 7.1 & 7.0 \\
\hline & $5 / 5 / 92$ & unsat. zone & & & 6,690 & 6,610 & 7.5 & 19.0 \\
\hline & $8 / 7 / 92$ & unsat. zone & & & 6,830 & 6,870 & 7.6 & -- \\
\hline & $9 / 8 / 92$ & unsat. zone & & & - & -- & -- & -- \\
\hline \multirow[t]{8}{*}{$5 S / 3 W-28 M 10 L Y$} & $2 / 25 / 91$ & unsat. zone & 10 & 1,420 & 8,550 & 7,810 & 7.6 & 19.5 \\
\hline & $6 / 5 / 91$ & unsat. zone & & & 4,740 & 8,310 & 7.3 & 19.5 \\
\hline & 7/5/91 & unsat. zone & & & - & $\ldots$ & -- & - \\
\hline & $8 / 12 / 91$ & unsat. zone & & & 8,920 & 8,810 & 7.2 & 21.5 \\
\hline & $2 / 11 / 92$ & unsat. zone & & & 8,520 & 8,450 & 7.1 & 16.0 \\
\hline & $5 / 5 / 92$ & unsat. zone & & & 8,740 & 8,630 & 7.4 & 19.0 \\
\hline & $8 / 7 / 92$ & unsat. zone & & & 9,060 & 9,090 & 7.8 & -- \\
\hline & $9 / 8 / 92$ & unsat. zone & & & - & - & - & -- \\
\hline $5 S / 3 W-28 M 11 L Y$ & $2 / 25 / 91$ & unsat. zone & 5 & 1,420 & 8,130 & 7,580 & 7.6 & 20.0 \\
\hline & $6 / 5 / 91$ & unsat. zone & & & 6,590 & 8,950 & 7.4 & 20.5 \\
\hline & $7 / 5 / 91$ & unsat. zone & & & -. & - & -- & - \\
\hline & $8 / 12 / 91$ & unsat. zone & & & 10,300 & 10,400 & 7.3 & 24.0 \\
\hline & $2 / 11 / 92$ & unsat. zone & & & 8,900 & 8,900 & 7.3 & 13.5 \\
\hline & $5 / 5 / 92$ & unsat. zone & & & 9,820 & 9,640 & 7.6 & 20.0 \\
\hline & $8 / 7 / 92$ & unsat. zone & & & 12,200 & 12,200 & 7.8 & - \\
\hline & $9 / 8 / 92$ & unsat. zone & & & - & -- & -- & -- \\
\hline $5 S / 3 W-29 Q 1$ & $7 / 7 / 58$ & -- & - & -- & -- & 10,900 & 6.3 & -- \\
\hline $5 S / 3 W-32 A 1$ & $3 / 11 / 94$ & - & 586.2 & 1,413 & -- & 7,000 & 7.0 & -- \\
\hline $5 S / 3 W-32 B 1$ & $11 / 5 / 93$ & -- & 218.1 & 1,414 & -- & 1,620 & 7.1 & -- \\
\hline $5 S / 3 W-32 C 1$ & $11 / 11 / 93$ & -- & 218.2 & 1,422 & - & 5,300 & 7.6 & -- \\
\hline $5 S / 3 W-32 G 1$ & $11 / 23 / 93$ & -- & 219.9 & 1,415 & - & 5,100 & 7.0 & -- \\
\hline $5 S / 3 W-32 G 2$ & $1 / 23 / 76$ & -- & -- & - & -- & 2,190 & 7.4 & -- \\
\hline
\end{tabular}


Table 2. Historical water-quality data for selected wells in the Winchester, Menifee, and south Perris ground-water subbasins, California-Continued

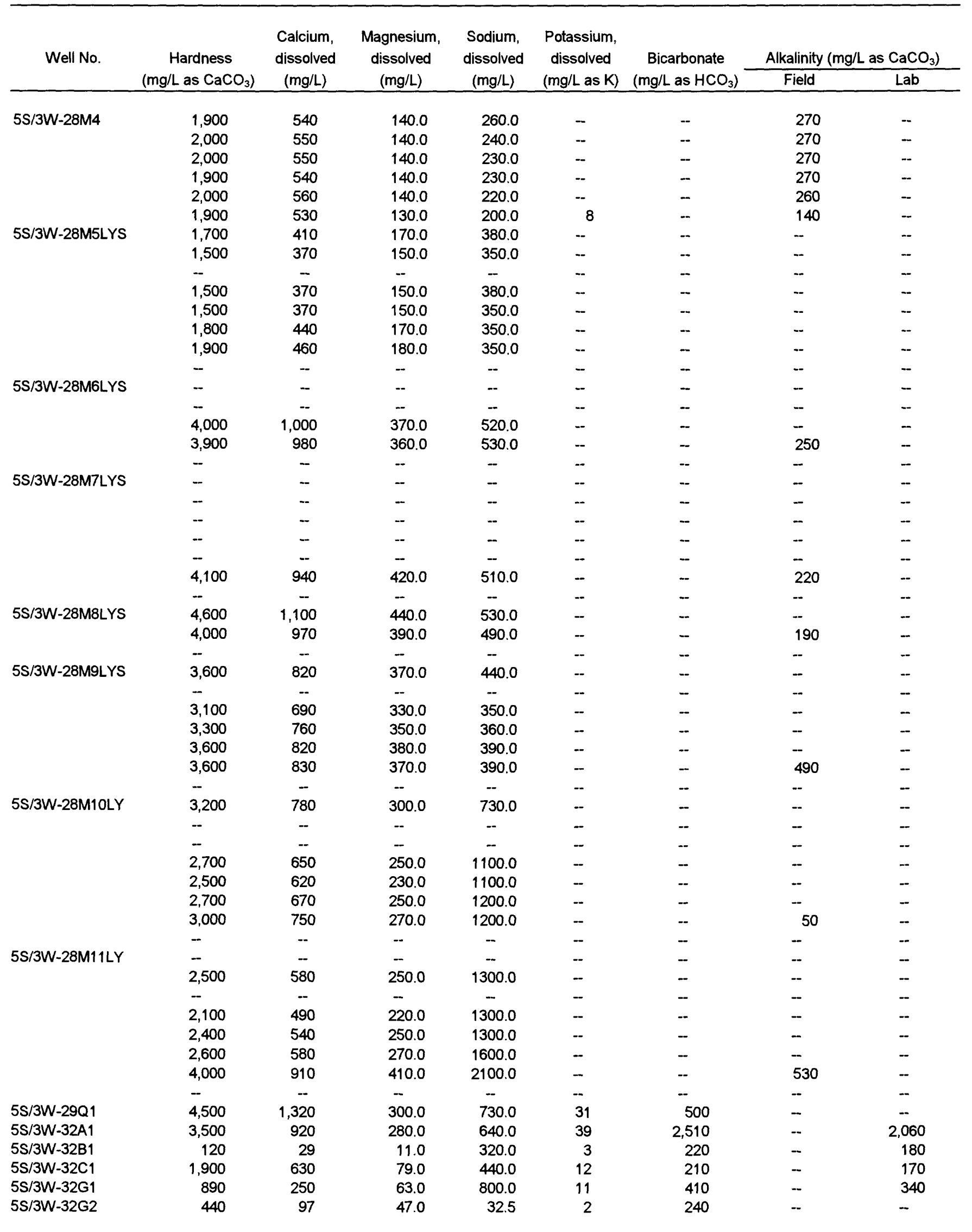


Table 2. Historical water-quality data for selected wells in the Winchester, Menifee, and south Perris ground-water subbasins, California--Continued

\begin{tabular}{|c|c|c|c|c|c|c|c|c|c|}
\hline \multirow{2}{*}{ Well No. } & \multirow{2}{*}{$\begin{array}{c}\begin{array}{c}\text { Sulfate, } \\
\text { dissolved } \\
\left(\mathrm{mg}^{2} \mathrm{~L} \text { as } \mathrm{SO}_{4}\right)\end{array} \\
\end{array}$} & \multirow{2}{*}{$\begin{array}{c}\text { Chloride, } \\
\text { dissolved } \\
\text { (mg/L) }\end{array}$} & \multirow{2}{*}{$\begin{array}{l}\text { Fluoride, } \\
\text { dissolved } \\
\text { (mg/L) }\end{array}$} & \multirow{2}{*}{$\begin{array}{l}\text { Bromide, } \\
\text { dissolved } \\
\text { (mg/L) }\end{array}$} & \multirow{2}{*}{$\begin{array}{c}\text { Silica, } \\
\text { dissolved } \\
\left(\mathrm{mg} / \mathrm{L} \text { as } \mathrm{SiO}_{2}\right) \\
\end{array}$} & \multicolumn{2}{|c|}{ Dissolved solids (mg/L) } & \multirow{2}{*}{$\begin{array}{c}\text { Nitrate, } \\
\text { dissolved } \\
\text { (mg/L as } \mathrm{N} \text { ) } \\
\end{array}$} & \multirow{2}{*}{$\begin{array}{c}\text { Nitrite } \\
\text { dissolved } \\
\text { (mg/L as } \mathrm{N})\end{array}$} \\
\hline & & & & & & (sum) & (residue) & & \\
\hline \multirow[t]{6}{*}{$5 S / 3 W-28 M 4$} & 840 & 1,000 & $<0.10$ & 1.8 & 59 & -- & 3,300 & - & $<0.010$ \\
\hline & 970 & 910 & 0.2 & - & 60 & -- & 3,360 & -- & $<0.010$ \\
\hline & 980 & 860 & 0.5 & 1.8 & 59 & -- & 3,250 & - & $<0.010$ \\
\hline & 660 & 700 & 0.3 & 1.8 & 57 & -- & 2,910 & - & $<0.010$ \\
\hline & 900 & 1,300 & 0.4 & 1.2 & 59 & -- & 3,440 & - & $<0.010$ \\
\hline & 880 & 903 & - & - & 52 & -- & -- & 6.2 & 0.40 \\
\hline \multirow[t]{7}{*}{ 5S/3W-28M5LYS } & 890 & 1,200 & 0.4 & 1.7 & 11 & -- & -- & - & - \\
\hline & 990 & 1,300 & 1.0 & 1.5 & 11 & -- & - & -- & $<0.010$ \\
\hline & - & - & - & -- & - & -- & - & - & -- \\
\hline & 1,000 & 950 & 0.5 & 1.4 & 11 & - & - & - & - \\
\hline & 90 & 99 & $<0.10$ & 0.5 & 11 & - & - & - & -- \\
\hline & 1,000 & 910 & $<0.10$ & 1.4 & 13 & -- & - & -- & $<0.010$ \\
\hline & -- & -- & -- & - & 15 & - & -- & -- & $<0.010$ \\
\hline \multirow{5}{*}{ 5S/3W-28M6LYS } & -- & -- & -- & - & -- & -- & -- & - & - \\
\hline & - & -- & -- & - & -- & -- & - & - & - \\
\hline & 660 & 1,300 & 0.4 & 4.2 & -- & -- & - & - & - \\
\hline & - & -- & -- & -- & 84 & - & -- & - & $<0.010$ \\
\hline & $\overline{-}$ & -- & -- & -- & 76 & - & -- & - & $<0.010$ \\
\hline \multirow{7}{*}{$5 S / 3 W-28 M 7 L Y S$} & 1,600 & 2,300 & $\overline{0.3}$ & $\overline{31}$ & - & -- & - & -- & -- \\
\hline & 1,900 & 2,500 & 0.7 & $\begin{array}{l}3.1 \\
3.8\end{array}$ & $\overline{-}$ & - & - & - & - \\
\hline & - & - & - & -- & - & $\overline{-}$ & $\begin{array}{l}-- \\
--\end{array}$ & $\begin{array}{l}-- \\
--\end{array}$ & - \\
\hline & 1,600 & 2,400 & 0.5 & 3.9 & -- & -- & - & - & 0.05 \\
\hline & -- & -- & - & - & -- & -- & -- & -- & 0.05 \\
\hline & -- & -- & -- & -- & 82 & -- & -- & -- & $<0.010$ \\
\hline & -- & - & - & -- & -. & -- & -- & -- & -- \\
\hline \multirow[t]{3}{*}{ 5S/3W-28M8LYS } & 1,400 & 2,600 & 1.0 & 0.5 & 84 & -- & -- & - & $<0.010$ \\
\hline & 1,200 & 2,600 & 0.6 & 4.5 & 83 & -- & -- & -- & $<0.010$ \\
\hline & -- & -- & -. & -- & -- & -- & -- & -- & -- \\
\hline \multirow{7}{*}{ 5S/3W-28M9LYS } & 2,500 & 1,400 & 1.4 & 1.0 & 89 & -- & -- & -- & 0.02 \\
\hline & - & -- & -- & - & - & -- & -- & -- & -- \\
\hline & 2,600 & 1,200 & 0.5 & 0.6 & 78 & - & -- & -- & 0.13 \\
\hline & 2,100 & 1,100 & 1.5 & 0.6 & 87 & -- & -- & -- & $<0.010$ \\
\hline & 2,100 & 1,100 & 0.2 & 0.4 & 88 & -- & -- & - & $<0.010$ \\
\hline & 2,100 & 1,200 & 0.9 & 0.4 & 90 & -- & -- & -- & $<0.010$ \\
\hline & -- & -- & -- & - & -- & -- & -- & -- & -- \\
\hline $5 S / 3 W-28 M 10 L Y$ & 2,800 & 1,800 & 0.7 & 0.4 & 87 & -- & - & - & -- \\
\hline & 2,400 & 1,700 & 2.5 & 0.4 & -- & -- & - & -- & $<0.010$ \\
\hline & -- & -- & -- & -- & -- & -- & - & -- & -- \\
\hline & 3,300 & 1,500 & 1.4 & 0.5 & 84 & - & -- & - & $<0.010$ \\
\hline & 2,500 & 1,400 & 0.6 & 0.4 & 80 & -- & -- & -- & $<0.010$ \\
\hline & 2,500 & 1,500 & 0.2 & 0.3 & 81 & -- & -- & -- & $<0.010$ \\
\hline & 2,700 & 1,600 & 0.7 & 0.3 & 91 & -- & - & -- & $<0.010$ \\
\hline & - & - & -- & -- & -- & -- & -- & -- & - \\
\hline $5 S / 3 W-28 M 11 L Y$ & 2,300 & 1,300 & 0.9 & 0.3 & -- & -- & -- & -- & - \\
\hline & 2,500 & 1,700 & 2.8 & 0.3 & 79 & -- & - & -- & 0.08 \\
\hline & - & -- & -- & -- & -- & -- & -- & -- & -- \\
\hline & 3,500 & 2,000 & 0.8 & 0.7 & 77 & -- & -- & -- & 0.01 \\
\hline & 2,500 & 1,700 & 0.6 & 0.3 & 77 & -- & -- & -- & $<0.010$ \\
\hline & 3,200 & 1,500 & 0.4 & 0.1 & 83 & -- & -- & -- & $<0.010$ \\
\hline & 3,900 & 2,200 & 0.8 & 0.4 & 110 & -- & -- & -- & $<0.010$ \\
\hline & - & -- & - & -- & - & -- & -- & - & -- \\
\hline $5 S / 3 W-29 Q 1$ & 1,000 & 3,230 & 0.0 & -- & -- & 7,100 & -- & 0.00 & -- \\
\hline $5 S / 3 W-32 A 1$ & 1,010 & 160 & 0.1 & -- & -- & -- & 3,660 & 0.45 & 0.10 \\
\hline $5 \mathrm{~S} / 3 \mathrm{~W}-32 \mathrm{~B} 1$ & 380 & 150 & 0.3 & -- & -- & -- & 1,100 & 2.9 & $<0.10$ \\
\hline $5 \mathrm{~S} / 3 \mathrm{~W}-32 \mathrm{C} 1$ & 700 & 1,400 & 0.3 & -- & -- & -- & 4,430 & 0.68 & $<0.10$ \\
\hline $5 S / 3 W-32 G 1$ & 930 & 970 & 0.3 & -- & -- & -- & 3,220 & 1.6 & $<0.10$ \\
\hline $5 \mathrm{~S} / 3 \mathrm{~W}-32 \mathrm{G} 2$ & 510 & 280 & 0.8 & -- & -- & -- & 1,560 & 6.8 & -- \\
\hline
\end{tabular}


Table 2. Historical water-quality data for selected wells in the Winchester, Menifee, and south Perris ground-water subbasins, California-Continued

\begin{tabular}{|c|c|c|c|c|c|c|c|c|c|}
\hline Well No. & $\begin{array}{c}\mathrm{NO}_{3}+\mathrm{NO}_{2} \\
\text { dissolved } \\
(\mathrm{mg} / \mathrm{L} \text { as } \mathrm{N})\end{array}$ & $\begin{array}{c}\text { Nitrogen } \\
\text { ammonia, } \\
\text { dissolved } \\
\text { (mg/L as } \mathrm{N} \text { ) }\end{array}$ & $\begin{array}{c}\text { Phosphorus, } \\
\text { dissolved } \\
\text { (mg/L) }\end{array}$ & $\begin{array}{c}\text { Phosphorus } \\
\text { ortho, } \\
\text { dissolved } \\
\text { (mg/L as P) }\end{array}$ & $\begin{array}{c}\text { Arsenic, } \\
\text { dissolved } \\
(\mu \mathrm{g} / \mathrm{L}) \\
\end{array}$ & $\begin{array}{c}\text { Barium, } \\
\text { dissolved } \\
(\mu \mathrm{g} / \mathrm{L})\end{array}$ & $\begin{array}{c}\text { Beryllium, } \\
\text { dissolved } \\
(\mu \mathrm{g} / \mathrm{L})\end{array}$ & $\begin{array}{c}\text { Boron, } \\
\text { dissolved } \\
(\mu \mathrm{g} / \mathrm{L})\end{array}$ & $\begin{array}{c}\text { Cadmium, } \\
\text { dissolved } \\
(\mu g / L)\end{array}$ \\
\hline \multirow[t]{6}{*}{$5 \mathrm{~S} / 3 \mathrm{~W}-28 \mathrm{M} 4$} & 5.0 & 0.02 & 0.40 & 0.26 & -- & 43 & 2.0 & 220 & - \\
\hline & 5.1 & 0.02 & 0.33 & 0.23 & - & 40 & $<2$ & 240 & -- \\
\hline & 5.1 & 0.03 & 0.29 & 0.23 & - & 41 & $<2$ & 230 & - \\
\hline & 5.2 & 0.03 & 0.27 & 0.26 & - & 31 & $<2$ & 240 & - \\
\hline & 5.1 & 0.11 & 0.27 & 0.24 & - & 34 & $<2$ & 260 & -- \\
\hline & 6.6 & 0.04 & -- & 0.25 & 1.0 & 32 & $<2$ & 260 & - \\
\hline \multirow[t]{8}{*}{ 5S/3W-28M5LYS } & $<0.05$ & - & -- & -- & -- & 63 & $<2$ & -- & - \\
\hline & $<0.05$ & - & - & $<0.01$ & - & 50 & $<2$ & -- & - \\
\hline & - & - & -- & -- & - & - & -- & - & -- \\
\hline & $<0.05$ & -- & -- & - & -- & 39 & $<2$ & -- & - \\
\hline & $<0.05$ & -- & -- & - & - & 29 & $<2$ & -- & -- \\
\hline & $<0.05$ & 0.14 & $<0.01$ & $<0.01$ & -- & 32 & $<2$ & -- & - \\
\hline & $<0.05$ & 0.10 & $<0.01$ & 0.01 & -- & 31 & $<2$ & -- & -- \\
\hline & -- & -- & -- & - & -- & - & -- & - & - \\
\hline \multirow[t]{5}{*}{ 5S/3W-28M6LYS } & -- & -- & -- & - & -- & -- & -- & - & -- \\
\hline & 13 & -- & - & -- & -- & - & -- & - & -- \\
\hline & 16 & 0.06 & 0.01 & $<0.01$ & -- & 37 & $<3$ & - & - \\
\hline & 17 & 0.11 & 0.02 & 0.01 & -- & 39 & $<3$ & - & - \\
\hline & - & - & - & -- & -- & - & - & -- & -- \\
\hline \multirow[t]{7}{*}{$5 S / 3 W-28 M 7 L Y S$} & 11 & - & - & -- & -- & - & - & - & -- \\
\hline & 10 & - & - & -- & - & - & - & - & - \\
\hline & -- & - & - & -- & - & - & - & - & - \\
\hline & 14 & 0.08 & $<0.01$ & $<0.01$ & - & - & - & -- & - \\
\hline & 14 & 0.12 & $<0.01$ & $<0.01$ & - & -- & - & - & -- \\
\hline & 14 & 0.12 & $<0.01$ & $<0.01$ & - & 38 & $<3$ & - & -- \\
\hline & - & - & -- & -- & - & - & - & -- & - \\
\hline \multirow[t]{3}{*}{$5 S / 3 W-28 M 8 L Y S$} & 17 & 0.11 & $<0.01$ & $<0.01$ & - & 20 & $<3$ & -- & -- \\
\hline & 18 & 0.12 & 0.01 & 0.01 & - & 53 & $<3$ & - & - \\
\hline & -- & -- & -- & -- & - & -- & -- & -- & - \\
\hline \multirow[t]{7}{*}{ 5S/3W-28M9LYS } & 5 & -- & -- & $<0.01$ & -- & 42 & $<3$ & - & - \\
\hline & - & -- & - & - & - & - & - & - & - \\
\hline & 2 & 0.22 & 0.03 & 0.03 & -- & 34 & $<2$ & - & - \\
\hline & 3 & 0.12 & 0.04 & 0.03 & -- & $<38$ & $<3$ & - & - \\
\hline & 9 & 0.08 & 0.02 & 0.03 & - & 15 & $<2$ & - & -- \\
\hline & 0.31 & 0.11 & 0.02 & 0.02 & - & 33 & $<2$ & -- & -- \\
\hline & - & - & - & -- & - & - & -- & - & -- \\
\hline \multirow[t]{8}{*}{$5 S / 3 W-28 M 10 L Y$} & 8.7 & -- & -- & - & - & 100 & $<10$ & -- & -- \\
\hline & 19 & 0.08 & 0.02 & 0.01 & -- & -- & -- & -- & -- \\
\hline & - & -- & - & - & - & -- & -- & -- & -- \\
\hline & 27 & 0.02 & 0.02 & 0.01 & -- & 29 & $<3$ & -- & - \\
\hline & 27 & 0.07 & 0.02 & 0.01 & -- & $<27$ & $<3$ & -- & - \\
\hline & 1.1 & 0.08 & $<0.01$ & $<0.01$ & - & 6.0 & $<3$ & -- & - \\
\hline & 0.20 & 0.09 & 0.01 & 0.01 & -- & 30 & $<3$ & -- & - \\
\hline & - & -- & -- & -- & -- & -- & - & -- & - \\
\hline \multirow[t]{8}{*}{ 5S/3W-28M11LY } & 5.0 & -- & -- & - & -- & - & - & - & - \\
\hline & 14 & 0.06 & 0.07 & 0.06 & - & 100 & $<10$ & - & - \\
\hline & - & -- & -- & -- & - & -- & -- & - & - \\
\hline & 26 & 0.03 & 0.05 & 0.04 & -- & 31 & $<3$ & - & - \\
\hline & 3.5 & 0.07 & 0.02 & 0.02 & - & $<29$ & $<3$ & - & - \\
\hline & 0.43 & 0.05 & $<0.01$ & 0.01 & - & $<5$ & $<3$ & - & -- \\
\hline & $<0.05$ & 0.12 & 0.02 & 0.02 & - & 30 & $<5$ & -- & -- \\
\hline & -- & - & - & - & -- & - & -- & - & - \\
\hline $5 S / 3 W-29 Q 1$ & -- & - & -- & - & - & - & -- & 300 & -- \\
\hline $5 S / 3 W-32 A 1$ & - & - & -. & - & $<5$ & 3,100 & $\ldots$ & -- & 1.0 \\
\hline $5 S / 3 W-32 B 1$ & - & -- & -- & - & $<5$ & $<100$ & -- & - & $<1$ \\
\hline $5 \mathrm{~S} / 3 \mathrm{~W}-32 \mathrm{C} 1$ & -- & -- & -- & - & $<5$ & 200 & -- & - & $<1$ \\
\hline $5 S / 3 W-32 G 1$ & -- & -- & -- & - & $<5$ & $<100$ & -- & - & $<1$ \\
\hline $5 S / 3 W-32 G 2$ & -- & $<1.0$ & -- & - & -- & - & -- & 800 & - \\
\hline
\end{tabular}


Table 2. Historical water-quality data for selected wells in the Winchester, Menifee, and south Perris ground-water subbasins, California-Continued

\begin{tabular}{|c|c|c|c|c|c|c|c|c|c|}
\hline Well No. & $\begin{array}{c}\text { Chromium, } \\
\text { dissolved } \\
(\mu \mathrm{g} / \mathrm{L})\end{array}$ & $\begin{array}{c}\text { Cobalt, } \\
\text { dissolved } \\
(\mu \mathrm{g} / \mathrm{L})\end{array}$ & $\begin{array}{l}\text { Copper, } \\
\text { dissolved } \\
(\mu \mathrm{g} / \mathrm{L}) \\
\end{array}$ & $\begin{array}{c}\text { Iron, } \\
\text { dissolved } \\
(\mu g / L) \\
\end{array}$ & $\begin{array}{c}\text { Lead, } \\
\text { dissolved } \\
(\mu g / L)\end{array}$ & $\begin{array}{c}\text { Lithium, } \\
\text { dissolved } \\
(\mu \mathrm{g} / \mathrm{L}) \\
\end{array}$ & $\begin{array}{c}\text { Manganese, } \\
\text { dissolved } \\
(\mu \mathrm{g} / \mathrm{L}) \\
\end{array}$ & $\begin{array}{c}\text { Molybdenum, } \\
\text { dissolved } \\
(\mu g / L)\end{array}$ & $\begin{array}{c}\text { Nickel, } \\
\text { dissolved } \\
(\mu \mathrm{g} / \mathrm{L})\end{array}$ \\
\hline \multirow[t]{6}{*}{$5 S / 3 W-28 M 4$} & $<20$ & $<9$ & $<30$ & 20 & 30 & 60 & 72 & $<30$ & $<30$ \\
\hline & $<20$ & $<9$ & $<30$ & 20 & 40 & 50 & 43 & $<30$ & $<30$ \\
\hline & $<20$ & $<9$ & $<30$ & 20 & $<30$ & 40 & 35 & 40 & $<30$ \\
\hline & $<20$ & $<9$ & $<30$ & $<9$ & $<30$ & 60 & 10 & $<30$ & $<30$ \\
\hline & $<20$ & $<9$ & $<30$ & 20 & $<30$ & 40 & 12 & $<30$ & $<30$ \\
\hline & 120 & $<9$ & $<30$ & 450 & $<30$ & 40 & 100 & $<30$ & $<30$ \\
\hline \multirow[t]{8}{*}{$5 S / 3 W-28 M 5 L Y S$} & $<20$ & $<9$ & $<30$ & 550 & $<30$ & 30 & 12,000 & 40 & $<30$ \\
\hline & $<20$ & $<9$ & $<30$ & 500 & $<30$ & 30 & 9,900 & $<30$ & $<30$ \\
\hline & - & -- & -- & -- & -- & - & -- & -- & -- \\
\hline & $<20$ & $<9$ & $<30$ & 600 & $<30$ & 20 & 9,200 & $<30$ & $<30$ \\
\hline & $<20$ & $<9$ & $<30$ & 150 & $<30$ & 30 & 8,600 & $<30$ & $<30$ \\
\hline & $<20$ & $<9$ & $<30$ & 3,500 & 30 & 30 & 8,000 & $<30$ & $<30$ \\
\hline & $<5$ & $<9$ & 30 & 4,300 & $<30$ & 10 & 7,000 & $<30$ & $<30$ \\
\hline & -- & -- & - & -- & - & -- & -- & - & - \\
\hline \multirow[t]{5}{*}{$5 \mathrm{~S} / 3 W-28 \mathrm{M} 6 \mathrm{LYS}$} & -- & -- & - & -- & -- & - & - & - & - \\
\hline & -- & -- & -- & -- & -- & -- & - & - & - \\
\hline & $<30$ & $<20$ & $<50$ & 20 & $<50$ & 20 & 3,600 & $<50$ & 100 \\
\hline & $<30$ & $<20$ & $<50$ & 30 & $<50$ & 70 & 2,600 & $<50$ & $<50$ \\
\hline & - & -- & -- & - & -- & - & -- & - & - \\
\hline \multirow[t]{7}{*}{$5 S / 3 W-28 M 7 L Y S$} & -- & -- & - & - & -- & -- & -- & - & - \\
\hline & -- & - & -- & - & - & -- & -- & - & -- \\
\hline & -- & -- & -- & - & -- & -- & - & -- & - \\
\hline & - & - & -- & - & - & -- & -- & - & - \\
\hline & -- & - & -- & -- & - & -- & -- & - & -- \\
\hline & $<30$ & $<20$ & $<50$ & $<15$ & $<50$ & 60 & 8,900 & 60 & 140 \\
\hline & -- & -- & - & - & -- & -. & - & - & -- \\
\hline \multirow{3}{*}{$5 S / 3 W-28 M 8 L Y S$} & $<30$ & 2 & 28 & 20 & $<1$ & 150 & 200 & $<50$ & 150 \\
\hline & $<30$ & $<20$ & $<50$ & $<15$ & $<50$ & 80 & 51 & $<50$ & 90 \\
\hline & - & - & - & - & -- & - & - & -- & - \\
\hline \multirow{7}{*}{ 5S/3W-28M9LYS } & $<30$ & $<20$ & $<50$ & 20 & $<50$ & 120 & 34 & $<50$ & $<50$ \\
\hline & - & - & -- & - & -- & - & -- & - & - \\
\hline & 4.0 & $<10$ & 80 & 50 & $<40$ & 60 & 75 & $<40$ & 70 \\
\hline & $<1$ & $<1$ & $<19$ & $<15$ & $<1$ & 20 & 360 & $<50$ & $<38$ \\
\hline & $<20$ & $<1$ & 10 & $<12$ & $<1$ & 60 & 37 & $<40$ & 11 \\
\hline & $<20$ & $<10$ & $<40$ & 30 & $<40$ & 50 & 44 & 50 & $<40$ \\
\hline & - & - & -- & -- & - & - & - & -- & - \\
\hline \multirow[t]{8}{*}{$5 \mathrm{~S} / 3 \mathrm{~W}-28 \mathrm{M} 10 \mathrm{LY}$} & 3.0 & 10 & 20 & 60 & 1 & 130 & 1,000 & 42 & 130 \\
\hline & - & -- & -- & - & - & -- & -- & -- & - \\
\hline & - & -- & -- & -. & -- & -- & - & -- & - \\
\hline & $<2$ & $<20$ & $<50$ & 30 & $<50$ & 220 & 52 & -- & $<50$ \\
\hline & $<1$ & $<1$ & $<20$ & $<15$ & $<1$ & 210 & 27 & $<50$ & $<38$ \\
\hline & $<30$ & $<1$ & 22 & 20 & $<1$ & 260 & $<5$ & $<50$ & 17 \\
\hline & $<30$ & $<20$ & $<50$ & $<15$ & $<50$ & 210 & 6 & 70 & $<50$ \\
\hline & - & - & - & - & -- & -- & - & - & -- \\
\hline \multirow[t]{8}{*}{ 5S/3W-28M11LY } & - & -- & -- & -- & - & -- & -- & -- & - \\
\hline & $<1$ & 10 & 43 & 10 & $<1$ & 130 & 60 & 29 & 80 \\
\hline & - & - & -- & -- & - & -- & - & -- & - \\
\hline & $<2$ & $<20$ & $<60$ & 23 & $<60$ & 110 & 37 & - & $<60$ \\
\hline & $<2$ & $<1$ & $<36$ & $<15$ & $<1$ & 90 & 19 & $<50$ & $<32$ \\
\hline & $<30$ & 1 & 47 & 20 & $<1$ & 180 & $<5$ & $<50$ & 17 \\
\hline & $<50$ & $<30$ & $<100$ & 100 & $<100$ & 180 & 11 & 110 & $<100$ \\
\hline & -. & -- & -- & - & -- & - & - & - & - \\
\hline $5 \mathrm{~S} / 3 \mathrm{~W}-29 \mathrm{Q} 1$ & -- & -- & -- & -- & - & - & - & - & -- \\
\hline $5 \mathrm{~S} / 3 \mathrm{~W}-32 \mathrm{~A} 1$ & $<10$ & -- & $<10$ & 40 & $<5$ & - & 3,500 & - & -- \\
\hline $5 \mathrm{~S} / 3 \mathrm{~W}-32 \mathrm{~B} 1$ & $<10$ & -- & $<10$ & $<20$ & $<5$ & -- & 30 & -- & -- \\
\hline $5 \mathrm{~S} / 3 \mathrm{~W}-32 \mathrm{C} 1$ & $<10$ & -- & $<10$ & 50 & $<5$ & -- & 530 & -- & -- \\
\hline $5 S / 3 W-32 G 1$ & $<10$ & -- & $<10$ & 250 & $<5$ & -- & 760 & -- & -- \\
\hline $5 \mathrm{~S} / 3 \mathrm{~W}-32 \mathrm{G} 2$ & - & -- & -- & - & - & - & - & - & - \\
\hline
\end{tabular}


Table 2. Historical water-quality data for selected wells in the Winchester, Menifee, and south Perris ground-water subbasins, California-Continued

\begin{tabular}{|c|c|c|c|c|c|c|}
\hline Well No. & $\begin{array}{c}\text { Selenium, } \\
\text { dissolved } \\
(\mu \mathrm{g} / \mathrm{L})\end{array}$ & $\begin{array}{c}\text { Silver, } \\
\text { dissolved } \\
(\mu \mathrm{g} / \mathrm{L})\end{array}$ & $\begin{array}{c}\text { Strontium, } \\
\text { dissolved } \\
(\mu \mathrm{g} / \mathrm{L})\end{array}$ & $\begin{array}{c}\text { Vanadium, } \\
\text { dissolved } \\
(\mu \mathrm{g} / \mathrm{L})\end{array}$ & $\begin{array}{c}\text { Zinc, } \\
\text { dissolved } \\
(\mu \mathrm{g} / \mathrm{L})\end{array}$ & Source of data \\
\hline \multirow[t]{6}{*}{$5 S / 3 W-28 M 4$} & 8.0 & 5.0 & 3,400 & 20 & $<9$ & USGS \\
\hline & 12 & 5.0 & 3,400 & 22 & $<9$ & USGS \\
\hline & 15 & $<3.0$ & 3,500 & 22 & $<9$ & USGS \\
\hline & 12 & $<3.0$ & 3,200 & $<18$ & 20 & USGS \\
\hline & 11 & $<3.0$ & 3,300 & 21 & $<9$ & USGS \\
\hline & 12 & $<3.0$ & 3,000 & $<18$ & 40 & USGS \\
\hline \multirow[t]{8}{*}{ 5S/3W-28M5LYS } & - & $<3.0$ & 2,300 & 29 & 930 & USGS \\
\hline & - & 4.0 & 2,200 & $<18$ & 90 & USGS \\
\hline & - & - & - & - & - & USGS \\
\hline & - & $<3.0$ & 2,100 & $<18$ & 20 & USGS \\
\hline & -- & $<3.0$ & 2,000 & $<18$ & 240 & USGS \\
\hline & - & 6.0 & 2,400 & $<18$ & 50 & USGS \\
\hline & - & $<3.0$ & 2,500 & $<9$ & 240 & USGS \\
\hline & - & - & - & -- & - & USGS \\
\hline \multirow[t]{5}{*}{ 5S/3W-28M6LYS } & - & -- & - & -- & - & USGS \\
\hline & - & - & - & - & - & USGS \\
\hline & - & $<5.0$ & 5,900 & 30 & 290 & USGS \\
\hline & - & $<5.0$ & 5,800 & 41 & 350 & USGS \\
\hline & -- & - & - & -- & - & USGS \\
\hline \multirow[t]{7}{*}{ 5S/3W-28M7LYS } & - & - & - & - & - & USGS \\
\hline & - & - & - & - & - & USGS \\
\hline & - & - & - & - & - & USGS \\
\hline & - & - & - & - & - & USGS \\
\hline & - & - & - & -- & - & USGS \\
\hline & -- & $<5.0$ & 6,100 & 41 & 680 & USGS \\
\hline & - & - & - & -- & - & USGS \\
\hline \multirow[t]{3}{*}{ 5S/3W-28M8LYS } & -- & $<1.0$ & 7,300 & $<30$ & 2,100 & USGS \\
\hline & - & $<5.0$ & 6,300 & $<30$ & 840 & USGS \\
\hline & - & - & - & - & - & USGS \\
\hline \multirow[t]{7}{*}{ 5S/3W-28M9LYS } & -- & $<5.0$ & 6,200 & $<30$ & 750 & USGS \\
\hline & - & - & - & - & - & USGS \\
\hline & - & $<4.0$ & 5,100 & $<24$ & 440 & USGS \\
\hline & - & $<1.0$ & 5,600 & $<27$ & 280 & USGS \\
\hline & - & $<1.0$ & 6,100 & $<24$ & 180 & USGS \\
\hline & - & $<4.0$ & 5,900 & 32 & 170 & USGS \\
\hline & - & - & - & - & - & USGS \\
\hline \multirow[t]{8}{*}{$5 S / 3 W-28 M 10 L Y$} & - & $<1.0$ & 5,300 & 66 & 1,000 & USGS \\
\hline & - & - & - & - & - & USGS \\
\hline & -- & - & - & -- & - & USGS \\
\hline & - & 7.0 & 5,300 & $<30$ & 470 & USGS \\
\hline & - & $<1.0$ & 4,900 & $<30$ & 480 & USGS \\
\hline & - & $<1.0$ & 5,300 & $<30$ & 210 & USGS \\
\hline & - & $<5.0$ & 5,700 & 35 & 170 & USGS \\
\hline & - & - & - & - & - & USGS \\
\hline \multirow[t]{8}{*}{$5 S / 3 W-28 M 11 L Y$} & - & - & - & -- & - & USGS \\
\hline & - & $<1.0$ & 4,900 & 32 & 600 & USGS \\
\hline & - & - & - & - & - & USGS \\
\hline & - & 9.0 & 4,100 & $<36$ & 480 & USGS \\
\hline & - & $<1.0$ & 4,200 & $<41$ & 490 & USGS \\
\hline & - & $<1.0$ & 4,700 & $<30$ & 230 & USGS \\
\hline & - & $<10$ & 6,800 & $<60$ & 190 & USGS \\
\hline & - & - & - & - & -- & USGS \\
\hline $5 S / 3 W-29 Q 1$ & - & - & - & -- & - & Babcock \\
\hline $5 S / 3 W-32 A 1$ & $<5$ & $<10$ & - & -- & 1,900 & Babcock \\
\hline $5 S / 3 W-32 B 1$ & $<5$ & $<10$ & -- & - & 100 & Babcock \\
\hline $5 S / 3 W-32 C 1$ & 5.0 & $<10$ & - & - & 430 & Babcock \\
\hline $5 S / 3 W-32 G 1$ & 12 & $<10$ & - & - & 400 & Babcock \\
\hline $5 S / 3 W-32 G 2$ & - & - & - & - & - & EMWD \\
\hline
\end{tabular}


Table 2. Historical water-quality data for selected wells in the Winchester, Menifee, and south Perris ground-water subbasins, California--Continued

\begin{tabular}{|c|c|c|c|c|c|c|c|c|}
\hline \multirow[t]{2}{*}{ Well No. } & \multirow[t]{2}{*}{ Date } & \multirow{2}{*}{$\begin{array}{l}\text { Water level } \\
\text { (ft blw LSD) }\end{array}$} & \multirow{2}{*}{$\begin{array}{c}\text { Well depth } \\
\text { (ft) }\end{array}$} & \multirow{2}{*}{$\begin{array}{c}\text { Altitude } \\
\text { of LSD (ft) } \\
\end{array}$} & \multicolumn{2}{|c|}{ Specific conductance $(\mu \mathrm{S} / \mathrm{cm})$} & \multirow{2}{*}{$\begin{array}{c}\mathrm{pH} \text { (lab) } \\
\text { (Std. units) }\end{array}$} & \multirow{2}{*}{$\begin{array}{c}\text { Water } \\
\text { temperature } \\
\left({ }^{\circ} \mathrm{C}\right) \\
\end{array}$} \\
\hline & & & & & Field & Lab & & \\
\hline $5 \mathrm{~S} / 3 \mathrm{~W}-32 \mathrm{H} 1$ & $10 / 29 / 93$ & - & 221 & 1,405 & -- & 1,800 & 6.7 & - \\
\hline $5 S / 3 W-32 L 1$ & $11 / 19 / 93$ & - & 221 & 1,412 & -- & 11,000 & 6.6 & - \\
\hline \multirow[t]{2}{*}{$5 S / 3 W-33 K 1$} & $4 / 6 / 94$ & - & 665 & 1,415 & - & 3,900 & 6.6 & - \\
\hline & $4 / 7 / 94$ & - & & & - & 3,600 & 7.7 & - \\
\hline $5 S / 3 W-33 R 2$ & $8 / 14 / 91$ & - & - & -- & - & 4,440 & 6.8 & - \\
\hline \multirow[t]{4}{*}{$5 S / 3 W-35 N 2$} & $7 / 15 / 92$ & -- & 587.6 & 1,425 & - & 4,000 & 6.3 & - \\
\hline & $7 / 15 / 92$ & - & & & - & 4,100 & 6.4 & - \\
\hline & $7 / 15 / 92$ & -- & & & - & 4,000 & 6.4 & - \\
\hline & $7 / 15 / 92$ & -- & & & - & 3,900 & 6.6 & - \\
\hline \multirow[t]{4}{*}{ 5S/3W-35P1 } & $5 / 9 / 56$ & - & 516 & 1,427 & -- & 2,530 & - & 21.0 \\
\hline & $4 / 23 / 59$ & - & & & - & 1,800 & 8.3 & 21.5 \\
\hline & $9 / 23 / 59$ & - & & & - & 1,470 & 7.8 & 230.0 \\
\hline & $5 / 17 / 68$ & - & & & - & 3,170 & 8.0 & - \\
\hline $5 S / 3 W-35 Q 1$ & $6 / 17 / 77$ & -- & 588 & -- & - & - & - & - \\
\hline $5 S / 3 W-35 R 1$ & $10 / 14 / 93$ & - & 600.3 & 1,425 & - & 3,800 & 6.2 & - \\
\hline \multirow[t]{4}{*}{ 5S/3W-36D1 } & $3 / 28 / 63$ & - & -- & - & -- & 1,610 & 7.4 & - \\
\hline & $11 / 5 / 64$ & - & & & - & 1,460 & 7.1 & - \\
\hline & $5 / 4 / 65$ & - & & & - & 1,300 & 7.4 & 15.0 \\
\hline & $5 / 17 / 68$ & - & & & - & 1,380 & 8.1 & - \\
\hline \multirow[t]{2}{*}{$5 \mathrm{~S} / 3 \mathrm{~W}-36 \mathrm{~K} 1$} & $2 / 27 / 62$ & - & 190 & 1,430 & - & 1,250 & 7.3 & 14.5 \\
\hline & $3 / 15 / 63$ & - & & & - & 760 & 7.4 & -- \\
\hline \multirow[t]{2}{*}{$5 S / 3 W-36 N 2$} & $8 / 27 / 91$ & - & 338.6 & 1,425 & - & 4,540 & 8.0 & - \\
\hline & $6 / 17 / 77$ & -- & & & - & - & - & 25.0 \\
\hline \multirow[t]{3}{*}{$5 S / 3 W-36 P 1$} & $12 / 17 / 53$ & -- & 128.7 & 1,425 & - & 2,850 & 6.5 & 20.5 \\
\hline & $8 / 10 / 55$ & - & & & -- & 2,330 & 6.2 & 22.0 \\
\hline & $9 / 18 / 56$ & - & & & - & 2,620 & -- & 23.0 \\
\hline $5 S / 3 W-36 P 2$ & $6 / 3 / 92$ & -- & 680.2 & 1,430 & - & 1,500 & 6.3 & - \\
\hline & $6 / 3 / 92$ & - & & & - & 1,800 & 6.3 & - \\
\hline & $6 / 2 / 92$ & - & & & - & 1,800 & 6.3 & -- \\
\hline & $6 / 2 / 92$ & -- & & & - & 2,800 & 6.5 & - \\
\hline & $6 / 2 / 92$ & -- & & & -- & 2,700 & 6.4 & - \\
\hline & $7 / 13 / 94$ & 104.71 & & & 2,580 & 2,620 & 6.4 & 23.5 \\
\hline $5 S / 3 W-36 Q 1$ & $5 / 8 / 58$ & - & 604 & - & - & 2,060 & 6.5 & 21.0 \\
\hline & $10 / 14 / 58$ & - & & & -- & 1,280 & 7.0 & 23.5 \\
\hline & $4 / 23 / 59$ & - & & & - & 3,730 & 8.0 & 21.0 \\
\hline & $3 / 25 / 60$ & - & & & - & 1,910 & 6.9 & 21.0 \\
\hline & $9 / 14 / 60$ & - & & & - & 1,170 & 7.8 & 22.0 \\
\hline & $4 / 13 / 61$ & - & & & $\ldots$ & 1,160 & 7.5 & 21.5 \\
\hline & $5 / 8 / 62$ & - & & & - & 1,260 & 7.0 & 23.5 \\
\hline & $11 / 1 / 62$ & - & & & - & 1,350 & 6.8 & 23.5 \\
\hline & $6 / 12 / 93$ & - & & & - & 1,340 & 6.6 & - \\
\hline & $4 / 17 / 64$ & -- & & & - & 1,410 & 7.1 & 21.0 \\
\hline & $3 / 30 / 65$ & - & & & -- & 1,480 & 6.5 & 22.0 \\
\hline 6S/3W-1D1 & $5 / 4 / 65$ & - & - & - & - & 1,110 & 7.8 & 18.0 \\
\hline $6 \mathrm{~S} / 3 \mathrm{~W}-1 \mathrm{D} 2$ & $4 / 22 / 75$ & -- & 500 & - & - & 1,460 & 8.0 & 24.0 \\
\hline $6 \mathrm{~S} / 3 W-1 \mathrm{E} 1$ & $4 / 20 / 77$ & -- & - & -- & - & - & - & - \\
\hline $6 \mathrm{~S} / 3 \mathrm{~W}-1 \mathrm{H} 1$ & $8 / 20 / 91$ & - & 369.5 & 1,430 & - & - & 6.9 & -- \\
\hline & $9 / 16 / 91$ & -- & & & - & - & -- & - \\
\hline & $10 / 15 / 91$ & -- & & & -- & - & -- & - \\
\hline $6 S / 3 W-1 J 2$ & $8 / 13 / 91$ & -- & 300 & 1,430 & - & 4,700 & 6.7 & - \\
\hline & $7 / 20 / 93$ & -- & & & 5,180 & 5,130 & 6.7 & 21.0 \\
\hline $6 \mathrm{~S} / 3 \mathrm{~W}-2 \mathrm{~A} 1$ & $11 / 22 / 93$ & - & 577 & 1,425 & - & 4,500 & 6.3 & - \\
\hline & $11 / 5 / 93$ & -- & & & -- & 5,300 & 6.4 & - \\
\hline & $11 / 4 / 93$ & -- & & & - & 2,800 & 6.3 & - \\
\hline & $11 / 4 / 93$ & - & & & - & 5,100 & 6.4 & - \\
\hline $6 S / 3 W-2 C 1$ & $4 / 22 / 75$ & - & 600 & 1,430 & - & 1,609 & 8.0 & 23.0 \\
\hline $6 S / 3 W-2 D 1$ & $11 / 4 / 93$ & - & 632.5 & 1,425 & - & 3,200 & 6.4 & - \\
\hline & $9 / 28 / 93$ & -- & & & - & 2,100 & 7.2 & - \\
\hline
\end{tabular}


Table 2. Historical water-quality data for selected wells in the Winchester, Menifee, and south Perris ground-water subbasins, California--Continued

\begin{tabular}{|c|c|c|c|c|c|c|c|c|}
\hline \multirow[t]{2}{*}{ Well No. } & \multirow{2}{*}{$\begin{array}{c}\text { Hardness } \\
\left(\mathrm{mg} / \mathrm{L} \text { as } \mathrm{CaCO}_{3}\right)\end{array}$} & \multirow{2}{*}{$\begin{array}{c}\text { Calcium, } \\
\text { dissolved } \\
(\mathrm{mg} / \mathrm{L})\end{array}$} & \multirow{2}{*}{$\begin{array}{c}\text { Magnesium, } \\
\text { dissolved } \\
(\mathrm{mg} / \mathrm{L})\end{array}$} & \multirow{2}{*}{$\begin{array}{c}\text { Sodium, } \\
\text { dissolved } \\
(\mathrm{mg} / \mathrm{L})\end{array}$} & \multirow{2}{*}{$\begin{array}{c}\text { Potassium, } \\
\text { dissolved } \\
(\mathrm{mg} / \mathrm{L} \text { as } \mathrm{K})\end{array}$} & \multirow{2}{*}{$\begin{array}{c}\text { Bicarbonate } \\
\left(\mathrm{mg} / \mathrm{L} \text { as } \mathrm{HCO}_{3}\right)\end{array}$} & \multicolumn{2}{|c|}{ Alkalinity $\left(\mathrm{mg} / \mathrm{L}\right.$ as $\left.\mathrm{CaCO}_{3}\right)$} \\
\hline & & & & & & & Field & Lab \\
\hline $5 \mathrm{~S} / 3 \mathrm{~W}-32 \mathrm{H} 1$ & 470 & 123 & 38.0 & 220.0 & 7 & 170 & - & 140 \\
\hline $5 S / 3 W-32 L 1$ & 3,600 & 1,070 & 210.0 & 1300.0 & 17 & 510 & - & 420 \\
\hline \multirow[t]{2}{*}{$5 S / 3 W-33 K 1$} & 1,300 & 320 & 110.0 & 310.0 & 8 & 350 & - & 290 \\
\hline & 1,200 & 330 & 98.0 & 350.0 & 8 & 440 & - & 360 \\
\hline 5S/3W-33R2 & 1,900 & 500 & 160.0 & 260.0 & 12 & 350 & - & 290 \\
\hline \multirow[t]{4}{*}{$5 \mathrm{~S} / 3 \mathrm{~W}-35 \mathrm{~N} 2$} & 1,100 & 350 & 110.0 & 450.0 & 11 & 420 & - & 340 \\
\hline & 1,200 & 320 & 100.0 & 480.0 & 11 & 430 & - & 350 \\
\hline & 1,200 & 320 & 97.0 & 430.0 & 11 & 470 & -- & 390 \\
\hline & 2,100 & 440 & 240.0 & 300.0 & 29 & 3,170 & - & 2,600 \\
\hline \multirow[t]{4}{*}{$5 S / 3 W-35 P 1$} & 670 & 170 & 57.0 & 260.0 & - & 310 & - & - \\
\hline & 370 & 120 & 19.0 & 280.0 & - & 300 & - & - \\
\hline & 260 & 78 & 16.0 & 240.0 & - & 270 & - & - \\
\hline & 700 & 190 & 55.0 & 410.0 & 6 & 310 & - & 260 \\
\hline $5 S / 3 W-35 Q 1$ & - & -- & - & - & - & - & - & - \\
\hline $5 S / 3 W-35 R 1$ & 1,500 & 370 & 130.0 & 320.0 & 11 & 550 & - & 450 \\
\hline \multirow[t]{4}{*}{$5 S / 3 W-36 D 1$} & 570 & 140 & 55.0 & 150.0 & 4 & 140 & -- & - \\
\hline & 490 & 120 & 47.0 & 120.0 & 5 & 130 & - & - \\
\hline & 420 & 100 & 39.0 & 117.0 & 5 & 140 & - & - \\
\hline & 460 & 110 & 42.0 & 97.0 & 4 & 120 & - & 100 \\
\hline \multirow[t]{2}{*}{$5 S / 3 W-36 K 1$} & 350 & 100 & 28.0 & 140.0 & 3. & 250 & - & - \\
\hline & 2 & 46 & 24.0 & 80.0 & 3 & 130 & - & - \\
\hline \multirow{2}{*}{$5 S / 3 W-36 N 2$} & 940 & 160 & 130.0 & 490.0 & 12 & 34 & - & 28 \\
\hline & -- & - & - & - & -- & - & - & - \\
\hline \multirow[t]{3}{*}{$5 S / 3 W-36 P 1$} & - & 320 & 72.0 & 260.0 & 8 & 370 & - & - \\
\hline & 1,000 & 120 & 38.0 & 160.0 & 4 & 360 & - & - \\
\hline & 940 & 230 & 87.0 & 190.0 & - & 490 & - & -- \\
\hline $5 S / 3 W-36 P 2$ & 580 & 140 & 58.0 & 98.0 & 7 & 700 & - & 580 \\
\hline & 740 & 170 & 76.0 & 110.0 & 8 & 1,040 & - & 850 \\
\hline & 680 & 160 & 71.0 & 120.0 & 8 & 980 & - & 800 \\
\hline & 1,100 & 230 & 130.0 & 260.0 & 11 & 1,910 & - & 1,570 \\
\hline & 1,100 & 230 & 120.0 & 230.0 & 10 & 1,790 & -- & 1,470 \\
\hline & 980 & 210 & 110.0 & 220.0 & 10 & - & 1,400 & -- \\
\hline $5 S / 3 W-36 Q 1$ & 650 & 170 & 55.0 & 210.0 & -- & 280 & - & -- \\
\hline & 1,500 & 380 & 120.0 & 720.0 & - & 200 & -- & -- \\
\hline & 1,200 & 380 & 74.0 & 370.0 & - & 300 & - & - \\
\hline & 610 & 170 & 42.0 & 180.0 & - & 310 & - & -- \\
\hline & 370 & 120 & 17.0 & 110.0 & 8 & 290 & - & - \\
\hline & 370 & 130 & 14.0 & 90.0 & 8 & 270 & - & -- \\
\hline & 420 & 130 & 23.0 & 98.0 & 6 & 270 & - & - \\
\hline & 430 & 140 & 22.0 & 100.0 & 6 & 250 & -- & - \\
\hline & 440 & 120 & 32.0 & 100.0 & 7 & 280 & - & -- \\
\hline & 460 & 140 & 27.0 & 100.0 & 6 & 280 & -- & - \\
\hline & 500 & 130 & 44.0 & 110.0 & 5 & 310 & - & - \\
\hline 6S/3W-1D1 & 360 & 66 & 46.0 & 110.0 & 7 & 230 & -- & - \\
\hline $6 S / 3 W-1 D 2$ & 510 & 130 & 47.0 & 110.0 & 6 & 370 & - & 300 \\
\hline 6S/3W-1E1 & - & -- & -- & - & -- & - & - & - \\
\hline $6 \mathrm{~S} / 3 \mathrm{~W}-1 \mathrm{H} 1$ & 1,700 & - & -- & 240.0 & - & - & - & 340 \\
\hline & 1,800 & -- & - & 270.0 & - & 350 & - & 290 \\
\hline & 1,700 & - & - & 280.0 & -- & 350 & - & 280 \\
\hline $6 \mathrm{~S} / 3 \mathrm{~W}-1 \mathrm{~J} 2$ & 1,800 & 500 & 140.0 & 340.0 & 12 & 310 & - & 260 \\
\hline & 2,000 & 570 & 140.0 & 400.0 & 10 & -- & 270 & -- \\
\hline $6 S / 3 W-2 A 1$ & 1,900 & 520 & 150.0 & 320.0 & 11 & 390 & - & 320 \\
\hline & 1,800 & 520 & 130.0 & 590.0 & 12 & 240 & - & 200 \\
\hline & 1,100 & 270 & 100.0 & 160.0 & 9 & 330 & - & 270 \\
\hline & 2,100 & 430 & 250.0 & 380.0 & 14 & 1,180 & - & 970 \\
\hline $6 \mathrm{~S} / 3 \mathrm{~W}-2 \mathrm{C} 1$ & 590 & 150 & 54.0 & 110.0 & 6 & 420 & -- & 340 \\
\hline $6 S / 3 W-2 D 1$ & 1,200 & 300 & 99.0 & 240.0 & 10 & 420 & - & 340 \\
\hline & 580 & 160 & 45.0 & 200.0 & 7 & 290 & - & 240 \\
\hline
\end{tabular}


Table 2. Historical water-quality data for selected wells in the Winchester, Menifee, and south Perris ground-water subbasins, California-Continued

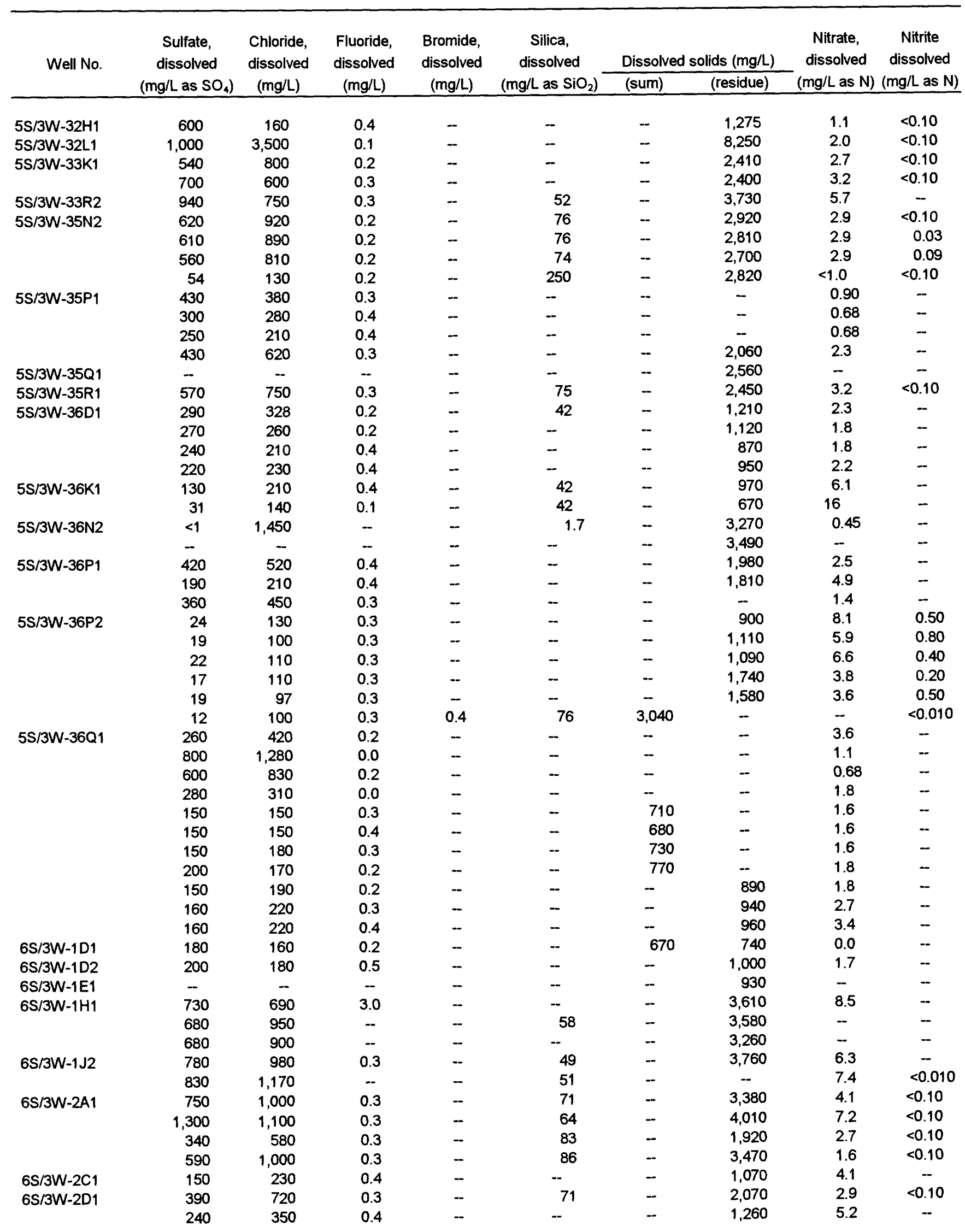


Table 2. Historical water-quality data for selected wells in the Winchester, Menifee, and south Perris ground-water subbasins, California--Continued

\begin{tabular}{|c|c|c|c|c|c|c|c|c|c|}
\hline Well No. & $\begin{array}{c}\mathrm{NO}_{3}+\mathrm{NO}_{2} \\
\text { dissolved } \\
(\mathrm{mg} / \mathrm{L} \text { as } \mathrm{N})\end{array}$ & $\begin{array}{c}\text { Nitrogen } \\
\text { ammonia, } \\
\text { dissolved } \\
\text { (mg/L as } \mathrm{N} \text { ) }\end{array}$ & $\begin{array}{c}\text { Phosphorus, } \\
\text { dissolved } \\
(\mathrm{mg} / \mathrm{L})\end{array}$ & $\begin{array}{l}\text { Phosphorus } \\
\text { ortho, } \\
\text { dissolved } \\
\text { (mg/L as P) }\end{array}$ & $\begin{array}{c}\text { Arsenic, } \\
\text { dissolved } \\
(\mu g / L)\end{array}$ & $\begin{array}{c}\text { Barium, } \\
\text { dissolved } \\
(\mu \mathrm{g} / \mathrm{L})\end{array}$ & $\begin{array}{c}\text { Beryllium, } \\
\text { dissolved } \\
(\mu g / L)\end{array}$ & $\begin{array}{c}\text { Boron, } \\
\text { dissolved } \\
(\mu g / L)\end{array}$ & $\begin{array}{c}\text { Cadmium, } \\
\text { dissolved } \\
(\mu g / L)\end{array}$ \\
\hline $5 \mathrm{~S} / 3 \mathrm{~W}-32 \mathrm{H} 1$ & -- & - & -- & - & $<5$ & $<100$ & - & - & $<1$ \\
\hline $5 S / 3 W-32 L 1$ & -- & -- & - & - & $<5$ & $<100$ & -- & - & 4.0 \\
\hline \multirow[t]{2}{*}{$5 S / 3 W-33 K 1$} & -- & -- & -- & - & $<10$ & $<100$ & $<1$ & - & $<1$ \\
\hline & - & - & - & -- & $<5$ & $<100$ & $<1$ & - & $<1$ \\
\hline $5 S / 3 W-33 R 2$ & -- & -- & -- & -- & $<10$ & $<100$ & - & $<100$ & $<1$ \\
\hline \multirow[t]{4}{*}{$5 S / 3 W-35 N 2$} & -- & -- & $<0.01$ & - & $<10$ & $<100$ & $<10$ & 700 & $<1$ \\
\hline & -- & -- & $<0.01$ & - & $<10$ & $<100$ & $<10$ & 700 & $<1$ \\
\hline & - & - & $<0.01$ & -- & $<10$ & $<100$ & $<10$ & 600 & $<1$ \\
\hline & - & -- & 0.02 & - & $<10$ & 1,200 & $<10$ & 800 & $<1$ \\
\hline \multirow{4}{*}{$5 S / 3 W-35 P 1$} & -- & - & - & - & -- & - & - & 230 & - \\
\hline & - & - & - & -- & - & - & - & 100 & - \\
\hline & - & - & - & - & - & -- & - & 200 & - \\
\hline & -- & -- & - & - & - & -- & -- & 600 & - \\
\hline $5 S / 3 W-35 Q 1$ & - & - & -- & - & -- & - & - & - & - \\
\hline $5 S / 3 W-35 R 1$ & - & - & -- & -- & $<5$ & $<100$ & - & -- & $<1$ \\
\hline \multirow[t]{4}{*}{$5 S / 3 W-36 D 1$} & - & - & - & - & -- & - & - & 120 & - \\
\hline & - & - & - & - & -- & -- & -- & 100 & -- \\
\hline & - & - & - & -- & - & - & - & 120 & -- \\
\hline & - & - & - & -- & - & - & - & 100 & -- \\
\hline \multirow[t]{2}{*}{$5 S / 3 W-36 K 1$} & - & -- & -- & - & - & -- & - & 150 & -- \\
\hline & - & - & -- & - & -- & -- & - & 150 & -- \\
\hline \multirow[t]{2}{*}{$5 S / 3 W-36 N 2$} & - & -- & -- & -- & $<10$ & $<100$ & - & 200 & $<1$ \\
\hline & - & -- & - & -- & - & -- & - & - & - \\
\hline \multirow[t]{3}{*}{$5 S / 3 W-36 P 1$} & - & - & - & - & - & - & - & 340 & - \\
\hline & -- & - & -- & - & - & - & - & 60 & - \\
\hline & -- & - & - & -- & -- & - & -- & 300 & - \\
\hline \multirow[t]{6}{*}{$5 \mathrm{~S} / 3 \mathrm{~W}-36 \mathrm{P} 2$} & - & -- & $<0.01$ & -- & $<10$ & 400 & $<10$ & 100 & $<1$ \\
\hline & - & - & $<0.01$ & - & $<10$ & 400 & $<10$ & 200 & $<1$ \\
\hline & - & - & 0.02 & -- & $<10$ & 400 & $<10$ & 200 & $<1$ \\
\hline & - & - & $<0.01$ & - & $<10$ & 800 & $<10$ & 400 & $<1$ \\
\hline & - & - & $<0.01$ & - & $<10$ & 700 & $<10$ & 300 & $<1$ \\
\hline & 3.8 & 0.02 & -- & 0.14 & 2.0 & 590 & $<0.5$ & 360 & - \\
\hline \multirow[t]{11}{*}{$5 S / 3 W-36 Q 1$} & - & - & -- & - & -- & -- & -- & 50 & -- \\
\hline & - & -- & - & - & -- & - & - & 1,400 & - \\
\hline & - & -- & - & -- & -- & - & - & 200 & -- \\
\hline & - & - & - & - & -- & -- & - & 400 & -- \\
\hline & - & -- & - & -- & -- & - & - & - & - \\
\hline & -- & - & -- & -- & -- & -- & - & 400 & -- \\
\hline & - & - & -- & - & -- & -- & - & 300 & -- \\
\hline & -- & - & -- & -- & - & - & -- & 200 & -- \\
\hline & - & -- & - & - & - & - & - & 100 & -- \\
\hline & - & - & - & - & -- & - & - & 100 & - \\
\hline & -- & - & -- & - & - & - & - & 120 & - \\
\hline 6S/3W-1D1 & - & -- & -- & - & - & - & - & 170 & - \\
\hline $6 S / 3 W-1 D 2$ & - & -- & -- & - & - & - & - & 40 & - \\
\hline $6 S / 3 W-1 E 1$ & - & -- & -- & - & - & - & - & - & - \\
\hline \multirow[t]{3}{*}{$6 S / 3 W-1 H 1$} & -- & -- & -- & - & - & - & - & - & 5.0 \\
\hline & -- & -- & -- & - & -- & -- & - & - & - \\
\hline & - & - & -- & - & - & - & - & - & - \\
\hline \multirow[t]{2}{*}{$6 S / 3 W-1 J 2$} & -- & - & -- & - & $<10$ & 100 & - & 200 & $<1$ \\
\hline & 7.4 & 0.08 & -- & 0.05 & 1.0 & 74 & $<2$ & 400 & - \\
\hline \multirow[t]{4}{*}{$6 S / 3 W-2 A 1$} & - & -- & - & -- & $<5$ & $<100$ & - & - & $<1$ \\
\hline & - & -- & - & - & $<5$ & $<100$ & - & - & $<1$ \\
\hline & -- & -- & -- & -- & $<5$ & 200 & - & - & $<1$ \\
\hline & - & - & - & - & $<5$ & 200 & - & - & 2.0 \\
\hline $6 \mathrm{~S} / 3 \mathrm{~W}-2 \mathrm{C} 1$ & - & -- & -- & - & -- & - & - & 90 & - \\
\hline \multirow[t]{2}{*}{$6 S / 3 W-2 D 1$} & - & - & -- & -- & $<5$ & $<100$ & -- & - & $<1$ \\
\hline & - & - & -- & - & $<5$ & $<100$ & - & - & $<1$ \\
\hline
\end{tabular}


Table 2. Historical water-quality data for selected wells in the Winchester, Menifee, and south Perris ground-water subbasins, California-Continued

\begin{tabular}{|c|c|c|c|c|c|c|c|c|c|}
\hline Well No. & $\begin{array}{l}\text { Chromium, } \\
\text { dissolved } \\
(\mu \mathrm{g} / \mathrm{L})\end{array}$ & $\begin{array}{c}\text { Cobalt, } \\
\text { dissolved } \\
(\mu g / L)\end{array}$ & $\begin{array}{c}\text { Copper, } \\
\text { dissolved } \\
(\mu \mathrm{g} / \mathrm{L})\end{array}$ & $\begin{array}{c}\text { Iron, } \\
\text { dissolved } \\
(\mu g / L)\end{array}$ & $\begin{array}{c}\text { Lead, } \\
\text { dissolved } \\
(\mu \mathrm{g} / \mathrm{L})\end{array}$ & $\begin{array}{l}\text { Lithium, } \\
\text { dissolved } \\
(\mu \mathrm{g} / \mathrm{L})\end{array}$ & $\begin{array}{c}\text { Manganese, } \\
\text { dissolved } \\
(\mu \mathrm{g} / \mathrm{L})\end{array}$ & $\begin{array}{c}\text { Molybdenum, } \\
\text { dissolved } \\
(\mu \mathrm{g} / \mathrm{L}) \\
\end{array}$ & $\begin{array}{c}\text { Nickel, } \\
\text { dissolved } \\
(\mu g / L)\end{array}$ \\
\hline $5 \mathrm{~S} / 3 \mathrm{~W}-32 \mathrm{H} 1$ & $<10$ & -- & $<10$ & $<20$ & $<5$ & -- & 30 & - & - \\
\hline $5 \mathrm{~S} / 3 \mathrm{~W}-32 \mathrm{~L} 1$ & $<10$ & -- & 50 & 540 & 150 & -- & 590 & -- & - \\
\hline \multirow[t]{2}{*}{$5 S / 3 W-33 K 1$} & $<10$ & -- & $<10$ & 120 & $<5$ & -- & 220 & -- & $<10$ \\
\hline & $<10$ & -- & $<10$ & 410 & $<5$ & -- & 140 & -- & $<10$ \\
\hline $5 S / 3 W-33 R 2$ & $<10$ & -- & 20 & 7,200 & 5.0 & -- & 200 & -- & - \\
\hline \multirow[t]{4}{*}{$5 S / 3 W-35 N 2$} & 10 & -- & $<10$ & 710 & $<10$ & -- & 100 & -- & 40 \\
\hline & $<10$ & -- & $<10$ & 760 & $<10$ & -- & 90 & -- & 20 \\
\hline & $<10$ & -- & $<10$ & 950 & 20 & -. & 180 & -- & 60 \\
\hline & 20 & -- & 30 & 21,000 & $<10$ & - & 3,600 & -- & 30 \\
\hline \multirow[t]{4}{*}{$5 S / 3 W-35 P 1$} & - & -- & -- & -- & -- & -- & - & -- & -. \\
\hline & -- & -- & -- & -- & -- & -- & -- & -- & - \\
\hline & -- & -- & -- & -- & -- & -- & -- & -- & -- \\
\hline & -- & -- & -- & -- & -- & -- & -- & -- & - \\
\hline $5 \mathrm{~S} / 3 W-35 \mathrm{Q} 1$ & -- & -- & -- & -- & -- & -- & -- & -- & -- \\
\hline $5 \mathrm{~S} / 3 W-35 R 1$ & $<10$ & - & 10 & 140 & $<5$ & .- & 120 & -- & - \\
\hline \multirow{4}{*}{$5 S / 3 W-36 D 1$} & - & -- & - & -- & -- & -. & - & - & - \\
\hline & -- & -- & -- & -- & -- & -- & -- & - & -- \\
\hline & -- & -- & -- & -- & -- & -- & -- & -- & - \\
\hline & -- & -- & -- & -- & -- & -- & -- & -- & -- \\
\hline \multirow[t]{2}{*}{$5 S / 3 W-36 K 1$} & -- & -- & -- & -- & -- & -- & -- & -- & -- \\
\hline & -- & -- & -- & - & -- & -- & - & -- & -- \\
\hline \multirow[t]{2}{*}{$5 \mathrm{~S} / 3 \mathrm{~W}-36 \mathrm{~N} 2$} & $<10$ & -- & 90 & 1,700 & $<5$ & -- & 510 & -- & -- \\
\hline & -. & - & -- & -- & - & - & - & -- & - \\
\hline \multirow[t]{3}{*}{$5 S / 3 W-36 P 1$} & -- & -- & -- & -- & -- & -- & -- & -- & -- \\
\hline & -. & _- & -- & - & - & -- & -- & -- & -- \\
\hline & -- & -- & -. & -- & -- & -. & -- & -- & -- \\
\hline \multirow[t]{6}{*}{$5 \mathrm{~S} / 3 \mathrm{~W}-36 \mathrm{P} 2$} & $<10$ & -- & $<10$ & 7,700 & $<5$ & -- & 730 & -- & $<20$ \\
\hline & $<10$ & -- & $<10$ & 8,100 & $<5$ & - & 650 & -- & $<20$ \\
\hline & $<10$ & -- & $<10$ & 5,600 & $<5$ & - & 830 & -- & $<20$ \\
\hline & $<10$ & -- & 10 & 15,000 & $<5$ & -- & 2,600 & -- & $<20$ \\
\hline & $<10$ & -- & 10 & 17,000 & 7.0 & -- & 2,100 & -- & $<20$ \\
\hline & $<5$ & 7 & $<10$ & 4 & $<10$ & 190 & 1,100 & $<10$ & $<10$ \\
\hline \multirow{11}{*}{$5 S / 3 W-36 Q 1$} & -- & -- & - & - & -- & - & - & - & -- \\
\hline & -- & - & -- & -- & -- & -- & -- & - & - \\
\hline & - & -- & -- & - & -- & -- & -- & -- & -- \\
\hline & -- & -- & -- & -- & -- & -- & -- & -- & - \\
\hline & -- & -- & -- & -- & -- & -- & -- & -- & -- \\
\hline & -- & -- & -- & - & -- & -- & -- & -- & -. \\
\hline & - & -- & -- & -- & -- & -. & -- & -- & -- \\
\hline & -- & - & -- & -- & -- & -- & -- & -- & -- \\
\hline & -- & -- & -- & -- & -- & - & -- & -- & -- \\
\hline & -- & -- & -- & -- & -- & -- & -- & -- & -- \\
\hline & - & -- & -- & -- & -- & -- & - & -- & -. \\
\hline 6S/3W-1D1 & -- & -- & -- & -- & -- & -- & -- & -- & -- \\
\hline $6 \mathrm{~S} / 3 \mathrm{~W}-1 \mathrm{D} 2$ & -- & - & -- & - & -- & -- & -- & -- & -- \\
\hline $6 S / 3 W-1 E 1$ & -- & - & -- & -- & - & -- & -- & -- & -- \\
\hline \multirow[t]{3}{*}{$6 \mathrm{~S} / 3 \mathrm{~W}-1 \mathrm{H} 1$} & 20 & -- & 110 & 110 & 3.0 & -- & 7 & -- & -- \\
\hline & -. & - & -- & - & - & - & -- & -- & -- \\
\hline & - & -- & -- & -- & -- & -- & -- & -- & - \\
\hline \multirow[t]{2}{*}{$6 S / 3 W-1 J 2$} & $<10$ & - & 20 & 50 & $<5$ & - & $<10$ & -- & -- \\
\hline & 80 & $<9$ & $<30$ & 140 & $<30$ & 50 & 46 & $<30$ & $<30$ \\
\hline \multirow{4}{*}{$6 \mathrm{~S} / 3 \mathrm{~W}-2 \mathrm{~A} 1$} & $<10$ & - & 20 & $<20$ & $<5$ & - & 70 & -- & -- \\
\hline & $<10$ & -- & $<10$ & 30 & $<5$ & -- & 50 & -- & -- \\
\hline & $<10$ & -- & $<10$ & 20 & $<5$ & -- & 300 & - & - \\
\hline & $<10$ & -- & 10 & $<20$ & $<5$ & -- & 1,300 & -- & -. \\
\hline & - & -- & - & - & -- & -- & - & -- & - \\
\hline \multirow{2}{*}{$6 S / 3 W-2 D 1$} & $<10$ & -- & 30 & $<20$ & $<5$ & -- & 140 & -- & -- \\
\hline & $<10$ & -- & $<10$ & $<20$ & 6.0 & -. & 80 & -- & -- \\
\hline
\end{tabular}


Table 2. Historical water-quality data for selected wells in the Winchester, Menifee, and south Perris ground-water subbasins, California-Continued

\begin{tabular}{|c|c|c|c|c|c|c|}
\hline Well No. & $\begin{array}{c}\text { Selenium, } \\
\text { dissolved } \\
(\mu \mathrm{g} / \mathrm{L})\end{array}$ & $\begin{array}{c}\text { Silver, } \\
\text { dissolved } \\
(\mu g / L)\end{array}$ & $\begin{array}{c}\text { Strontium, } \\
\text { dissolved } \\
(\mu \mathrm{g} / \mathrm{L})\end{array}$ & $\begin{array}{c}\text { Vanadium, } \\
\text { dissolved } \\
(\mu \mathrm{g} / \mathrm{L})\end{array}$ & $\begin{array}{c}\text { Zinc, } \\
\text { dissolved } \\
(\mu \mathrm{g} / \mathrm{L})\end{array}$ & Source of data \\
\hline $5 \mathrm{~S} / 3 \mathrm{~W}-32 \mathrm{H} 1$ & $<5$ & $<10$ & - & - & 120 & Babcock \\
\hline $5 \mathrm{~S} / 3 \mathrm{~W}-32 \mathrm{~L} 1$ & 21 & $<10$ & - & - & 770 & Babcock \\
\hline \multirow[t]{2}{*}{$5 S / 3 W-33 K 1$} & 7.0 & $<10$ & - & - & 150 & Babcock - 280'-300' \\
\hline & 7.0 & $<10$ & - & -- & 70 & Babcock - $170^{\circ}-190^{\prime}$ \\
\hline $5 S / 3 W-33 R 2$ & $<5$ & $<10$ & - & - & 1,100 & Babcock \\
\hline \multirow[t]{4}{*}{$5 S / 3 W-35 N 2$} & 5.0 & $<10$ & 1,500 & -- & 2,800 & Babcock - 250'-350' \\
\hline & 5.0 & $<10$ & 1,500 & - & 4,400 & Babcock - $400^{\prime}-440^{\prime}$ \\
\hline & $<5$ & $<10$ & 1,400 & -- & 6,400 & Babcock - 480'-520' \\
\hline & $<5$ & $<10$ & 2,400 & -- & 16,000 & Babcock - 580'-600' \\
\hline \multirow[t]{4}{*}{$5 S / 3 W-35 P 1$} & -- & -- & -- & -- & - & RCFCD \\
\hline & - & - & -- & -. & - & RCFCD \\
\hline & -- & -- & -- & - & - & RCFCD \\
\hline & - & -- & -- & - & -- & RCFCD \\
\hline $5 S / 3 W-35 Q 1$ & -- & -- & - & -- & - & EMWD \\
\hline 5S/3W-35R1 & 6.0 & $<10$ & - & - & 270 & Babcock \\
\hline \multirow[t]{4}{*}{$5 S / 3 W-36 D 1$} & - & -- & - & -- & - & RCFCD \\
\hline & -- & -- & - & -- & - & RCFCD \\
\hline & -- & -- & - & -- & - & RCFCD \\
\hline & - & - & - & - & -- & RCFCD \\
\hline \multirow[t]{2}{*}{$5 \mathrm{~S} / 3 \mathrm{~W}-36 \mathrm{~K} 1$} & -- & - & - & -- & - & RCFCD \\
\hline & - & - & - & -- & -- & RCFCD \\
\hline \multirow[t]{2}{*}{$5 S / 3 W-36 N 2$} & $<5$ & $<10$ & -- & -- & - & Babcock \\
\hline & -- & -- & -- & - & -- & EMWD \\
\hline \multirow[t]{3}{*}{$5 \mathrm{~S} / 3 \mathrm{~W}-36 \mathrm{P} 1$} & - & - & - & - & - & RCFCD \\
\hline & - & -- & -- & -- & -- & RCFCD \\
\hline & - & - & - & -- & - & RCFCD \\
\hline \multirow[t]{6}{*}{$5 S / 3 W-36 P 2$} & $<5$ & $<10$ & 600 & -- & 6,600 & Babcock - $400^{\prime}-440^{\prime}$ \\
\hline & $<5$ & $<10$ & 700 & - & 7,000 & Babcock - 460'-500' \\
\hline & $<5$ & $<10$ & 700 & - & 12,000 & Babcock - 520'-560' \\
\hline & $<5$ & 20 & 1,100 & -- & 5,600 & Babcock - 580'-620' \\
\hline & $<5$ & $<5$ & 900 & - & 30,000 & Babcock - $640^{\prime}-680^{\prime}$ \\
\hline & -- & 2.0 & 1,000 & 19 & 20 & USGS \\
\hline \multirow[t]{11}{*}{ 5S/3W-36Q1 } & - & -- & - & -- & -- & RCFCD \\
\hline & - & -- & -- & - & - & RCFCD \\
\hline & - & - & -- & -- & - & RCFCD \\
\hline & - & -- & -- & - & -- & RCFCD \\
\hline & - & -- & -- & -- & -- & RCFCD \\
\hline & -- & - & -- & -- & -- & RCFCD \\
\hline & - & - & -- & -- & - & RCFCD \\
\hline & - & -- & -- & -- & -- & RCFCD \\
\hline & - & -- & - & - & -- & RCFCD \\
\hline & -- & - & - & - & - & RCFCD \\
\hline & - & - & -- & -- & -- & RCFCD \\
\hline 6S/3W-1D1 & - & - & -- & -- & -- & RCFCD \\
\hline 6S/3W-1D2 & - & -- & - & - & - & DWR \\
\hline 6S/3W-1E1 & - & - & - & -- & -- & EMWD \\
\hline \multirow[t]{3}{*}{$6 S / 3 W-1 H 1$} & -- & - & - & - & 2,100 & WNTL \\
\hline & - & - & -- & -- & - & Babcock \\
\hline & - & -- & - & - & - & Babcock \\
\hline \multirow[t]{2}{*}{$6 S / 3 W-1 \sqrt{ } 2$} & $<5$ & $<10$ & - & - & 10 & Babcock \\
\hline & 9.0 & $<3.0$ & 2,400 & $<18$ & 13 & USGS \\
\hline \multirow[t]{4}{*}{$6 S / 3 W-2 A 1$} & 6.0 & $<10$ & - & -- & 270 & Babcock - Composite \\
\hline & 6.0 & $<10$ & - & $\ldots$ & 60 & Babcock - $180^{\prime}-200^{\prime}$ \\
\hline & $<5$ & $<10$ & - & -- & 90 & Babcock - 300'-440' \\
\hline & $<5$ & $<10$ & - & - & 260 & Babcock - 550'-570' \\
\hline & -- & - & -- & - & - & DWR \\
\hline \multirow[t]{2}{*}{$6 \mathrm{~S} / 3 \mathrm{~W}-2 \mathrm{D} 1$} & $<5$ & $<10$ & - & - & 420 & Babcock - Composite \\
\hline & $<5$ & $<10$ & -- & -- & 40 & Babcock - 205'-225' \\
\hline
\end{tabular}


Table 2. Historical water-quality data for selected wells in the Winchester, Menifee, and south Perris ground-water subbasins, California-Continued

\begin{tabular}{|c|c|c|c|c|c|c|c|c|}
\hline \multirow[t]{2}{*}{ Well No. } & \multirow[t]{2}{*}{ Date } & \multirow{2}{*}{$\begin{array}{l}\text { Water level } \\
\text { (ft blw LSD) }\end{array}$} & \multirow{2}{*}{$\begin{array}{l}\text { Well depth } \\
\text { (ft) }\end{array}$} & \multirow{2}{*}{$\begin{array}{c}\text { Altitude } \\
\text { of LSD (ft) }\end{array}$} & \multicolumn{2}{|c|}{ Specific conductance $(\mu \mathrm{S} / \mathrm{cm})$} & \multirow{2}{*}{$\begin{array}{c}\mathrm{pH} \text { (lab) } \\
\text { (Std. units) }\end{array}$} & \multirow{2}{*}{ 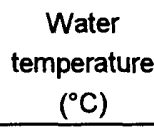 } \\
\hline & & & & & Field & Lab & & \\
\hline & 9/27/93 & -- & & & -- & 2,500 & 6.9 & -- \\
\hline & 9/27/93 & -- & & & -- & 1,470 & 8.1 & - \\
\hline \multirow[t]{3}{*}{$6 S / 3 W-2 E 1$} & $11 / 13 / 93$ & -- & 651.3 & 1,425 & -- & 1,690 & 6.4 & -- \\
\hline & 10/17/93 & -- & & & -- & 1,740 & 6.9 & - \\
\hline & 10/18/93 & -- & & & - & 1,400 & 7.8 & -- \\
\hline \multirow{4}{*}{$6 S / 3 W-2 F 1$} & $3 / 15 / 63$ & -- & - & 1,425 & -- & 650 & 7.1 & - \\
\hline & $11 / 5 / 64$ & - & & & - & 590 & 6.9 & -- \\
\hline & $5 / 4 / 65$ & -- & & & -- & 595 & 7.3 & -- \\
\hline & $5 / 17 / 68$ & -- & & & -- & 797 & 7.7 & -- \\
\hline \multirow[t]{3}{*}{$6 S / 3 W-2 G 1$} & $6 / 4 / 91$ & - & 625 & 1,426 & -- & -- & 8.0 & -- \\
\hline & $6 / 12 / 91$ & -- & & & -- & 1,240 & 7.4 & - \\
\hline & $6 / 14 / 91$ & -- & & & -- & - & - & - \\
\hline \multirow{3}{*}{$6 S / 3 W-2 G 2$} & $6 / 4 / 91$ & - & 622 & 1,428 & -- & - & 7.7 & - \\
\hline & $6 / 12 / 91$ & -- & & & - & 1,640 & 7.3 & -- \\
\hline & $6 / 14 / 91$ & -- & & & - & -- & -- & -. \\
\hline \multirow[t]{3}{*}{$6 \mathrm{~S} / 3 \mathrm{~W}-2 \mathrm{H} 2$} & $6 / 4 / 91$ & - & 565 & 1,428 & - & -- & 8.1 & -- \\
\hline & $6 / 12 / 91$ & -- & & & -- & 1,280 & 7.4 & -- \\
\hline & $6 / 14 / 91$ & - & & & -- & -- & -- & -- \\
\hline \multirow[t]{3}{*}{$6 \mathrm{~S} / 3 \mathrm{~W}-3 \mathrm{C} 1$} & $10 / 1 / 75$ & -- & 600 & 1,425 & -- & 3,500 & 6.3 & - \\
\hline & $10 / 10 / 75$ & -- & & & - & 3,300 & 6.1 & -- \\
\hline & $7 / 1 / 91$ & -- & & & -- & 3,210 & 6.0 & - \\
\hline \multirow[t]{2}{*}{$6 S / 3 W-3 C 2$} & $5 / 17 / 67$ & -- & - & - & - & 1,160 & 8.0 & - \\
\hline & $4 / 22 / 75$ & -- & & & - & 2,880 & 7.7 & 23.0 \\
\hline \multirow[t]{2}{*}{$6 \mathrm{~S} / 3 \mathrm{~W}-3 \mathrm{H} 2$} & $4 / 20 / 77$ & - & 468 & 1,430 & - & -- & -- & - \\
\hline & $6 / 7 / 91$ & -- & & & -- & 1,910 & 6.9 & -- \\
\hline $6 \mathrm{~S} / 3 \mathrm{~W}-3 \mathrm{~L} 1$ & $7 / 20 / 93$ & 107.09 & 200 & 1.460 & 600 & 614 & 7.2 & 22.0 \\
\hline \multirow[t]{2}{*}{$6 S / 3 W-4 K 1$} & $1 / 26 / 53$ & - & -- & 1,452 & -- & 980 & - & - \\
\hline & $3 / 15 / 63$ & -- & & & -- & 550 & 7.3 & 19.5 \\
\hline $6 S / 3 W-8 R 1$ & $12 / 10 / 93$ & -- & 250 & 1,525 & 1,200 & 1,190 & 7.8 & 21.0 \\
\hline $6 S / 3 W-9 E 1$ & $12 / 10 / 93$ & - & 150 & 1,500 & 680 & 690 & 7.5 & 21.5 \\
\hline $6 \mathrm{~S} / 3 \mathrm{~W}-9 \mathrm{G} 1$ & $12 / 10 / 93$ & -- & - & 1,485 & 1,240 & 1,250 & 7.6 & 21.0 \\
\hline
\end{tabular}


Table 2. Historical water-quality data for selected wells in the Winchester, Menifee, and south Perris ground-water subbasins, California-Continued

\begin{tabular}{|c|c|c|c|c|c|c|c|c|}
\hline \multirow[t]{2}{*}{ Well No. } & \multirow{2}{*}{$\begin{array}{c}\text { Hardness } \\
\left(\mathrm{mg} / \mathrm{L} \text { as } \mathrm{CaCO}_{3}\right)\end{array}$} & \multirow{2}{*}{$\begin{array}{c}\text { Calcium, } \\
\text { dissolved } \\
\text { (mg/L) }\end{array}$} & \multirow{2}{*}{$\begin{array}{c}\text { Magnesium, } \\
\text { dissolved } \\
(\mathrm{mg} / \mathrm{L})\end{array}$} & \multirow{2}{*}{$\begin{array}{c}\text { Sodium, } \\
\text { dissolved } \\
(\mathrm{mg} / \mathrm{L})\end{array}$} & \multirow{2}{*}{$\begin{array}{c}\text { Potassium, } \\
\text { dissolved } \\
\text { (mg/L as K) }\end{array}$} & \multirow{2}{*}{$\begin{array}{c}\text { Bicarbonate } \\
\left(\mathrm{mg} / \mathrm{L} \text { as } \mathrm{HCO}_{3}\right)\end{array}$} & \multicolumn{2}{|c|}{ Alkalinity (mg/L as $\mathrm{CaCO}_{3}$} \\
\hline & & & & & & & Field & Lab \\
\hline & 950 & 240 & 83.0 & 120.0 & 9 & 370 & - & 300 \\
\hline & 440 & 84 & 55.0 & 150.0 & 12 & 580 & -- & 480 \\
\hline \multirow[t]{3}{*}{$6 \mathrm{~S} / 3 \mathrm{~W}-2 \mathrm{E} 1$} & 630 & 160 & 52.0 & 110.0 & 7 & 490 & - & 400 \\
\hline & 650 & 170 & 54.0 & 120.0 & 8 & 610 & - & 500 \\
\hline & 500 & 120 & 47.0 & 130.0 & 9 & 790 & - & 650 \\
\hline \multirow[t]{4}{*}{$6 \mathrm{~S} / 3 \mathrm{~W}-2 \mathrm{~F} 1$} & 180 & 43 & 18.0 & 57.0 & 2 & 140 & - & - \\
\hline & 190 & 47 & 17.0 & 52.0 & 3 & 130 & - & - \\
\hline & 190 & 51 & 14.0 & 56.0 & 3 & 140 & - & -- \\
\hline & 250 & 61 & 24.0 & 63.0 & 3 & 160 & - & 130 \\
\hline \multirow[t]{3}{*}{$6 \mathrm{~S} / 3 W-2 \mathrm{G} 1$} & 320 & 85 & 25.0 & 120.0 & 4 & 180 & - & -- \\
\hline & -- & 88 & 27.0 & 110.0 & 5 & 200 & - & 160 \\
\hline & -- & - & -- & -- & - & - & - & -- \\
\hline \multirow[t]{3}{*}{$6 \mathrm{~S} / 3 \mathrm{~W}-2 \mathrm{G} 2$} & 490 & 140 & 35.0 & 150.0 & 5 & 280 & - & - \\
\hline & - & 130 & 41,0 & 130.0 & 6 & 270 & - & 220 \\
\hline & -- & -- & -- & - & - & -- & - & - \\
\hline \multirow[t]{3}{*}{$6 \mathrm{~S} / 3 \mathrm{~W}-2 \mathrm{H} 2$} & 370 & 100 & 28.0 & 98.0 & 5 & 210 & -- & -- \\
\hline & - & 110 & 34.0 & 96.0 & 5 & 230 & -- & 190 \\
\hline & -- & - & - & - & - & -- & - & -- \\
\hline \multirow[t]{3}{*}{$6 \mathrm{~S} / 3 W-3 \mathrm{C} 1$} & 1,500 & 400 & 130.0 & 200.0 & 11 & 780 & - & -- \\
\hline & 1,600 & 460 & 96.0 & 240.0 & 16 & 880 & -- & - \\
\hline & 1,400 & 350 & 120.0 & 190.0 & 10 & 960 & - & 790 \\
\hline \multirow[t]{2}{*}{$6 S / 3 W-3 C 2$} & 360 & 89 & 33.0 & 97.0 & 13 & 150 & -- & - \\
\hline & 1,200 & 300 & 100.0 & 130.0 & 10 & 400 & - & 320 \\
\hline \multirow[t]{2}{*}{$6 \mathrm{~S} / 3 \mathrm{~W}-3 \mathrm{H} 2$} & - & - & -- & - & - & - & - & - \\
\hline & 670 & 180 & 55.0 & 120.0 & 3 & 240 & - & 200 \\
\hline $6 S / 3 W-3 L 1$ & 180 & 53 & 12.0 & 46.0 & 3 & - & 98 & - \\
\hline \multirow[t]{2}{*}{$6 S / 3 W-4 K 1$} & - & 74 & 21.0 & 77.0 & - & 270 & - & - \\
\hline & 150 & 32 & 18.0 & 53.0 & 4 & 230 & -- & - \\
\hline $6 S / 3 W-8 R 1$ & 360 & 98 & 28.0 & 91.0 & 2 & - & 190 & - \\
\hline $6 \mathrm{~S} / 3 \mathrm{~W}-9 \mathrm{E} 1$ & 190 & 50 & 16.0 & 60.0 & 2 & - & 120 & - \\
\hline $6 S / 3 W-9 G 1$ & 380 & 100 & 32.0 & 100.0 & 2 & - & 240 & - \\
\hline
\end{tabular}


Table 2. Historical water-quality data for selected wells in the Winchester, Menifee, and south Perris ground-water subbasins, California-Continued

\begin{tabular}{|c|c|c|c|c|c|c|c|c|c|}
\hline \multirow[t]{2}{*}{ Well No. } & \multirow{2}{*}{$\begin{array}{c}\text { Sulfate, } \\
\text { dissolved } \\
\left(\mathrm{mg} / \mathrm{L} \text { as } \mathrm{SO}_{4}\right)\end{array}$} & \multirow{2}{*}{$\begin{array}{l}\text { Chloride, } \\
\text { dissolved } \\
(\mathrm{mg} / \mathrm{L}) \\
\end{array}$} & \multirow{2}{*}{$\begin{array}{c}\text { Fluoride, } \\
\text { dissolved } \\
(\mathrm{mg} / \mathrm{L})\end{array}$} & \multirow{2}{*}{$\begin{array}{c}\text { Bromide, } \\
\text { dissolved } \\
(\mathrm{mg} / \mathrm{L})\end{array}$} & \multirow{2}{*}{ 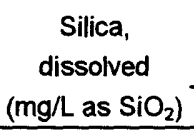 } & \multicolumn{2}{|c|}{ Dissolved solids (mg/L) } & \multirow{2}{*}{ 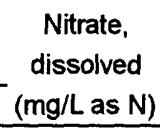 } & \multirow{2}{*}{$\begin{array}{c}\begin{array}{c}\text { Nitrite } \\
\text { dissolved } \\
\text { (mg/L as N) }\end{array} \\
\end{array}$} \\
\hline & & & & & & (sum) & (residue) & & \\
\hline & 230 & 510 & 0.2 & -- & -- & -- & 1,650 & 1.8 & $<0.10$ \\
\hline & 180 & 95 & 0.3 & -- & -- & -- & 890 & 0.23 & 0.10 \\
\hline \multirow[t]{3}{*}{$6 \mathrm{~S} / 3 \mathrm{~W}-2 \mathrm{E} 1$} & 160 & 210 & 0.3 & -- & -- & -- & 1,080 & 3.8 & - \\
\hline & 120 & 200 & 0.2 & - & 71 & - & 1,050 & 3.4 & $<0.10$ \\
\hline & 36 & 69 & 0.2 & -- & 62 & -. & 830 & 1.6 & $<0.10$ \\
\hline \multirow[t]{4}{*}{$6 S / 3 W-2 F 1$} & 57 & 76 & 0.1 & -- & 47 & - & 410 & 4.3 & -- \\
\hline & 60 & 80 & 0.2 & -- & -- & - & 420 & 4.7 & -- \\
\hline & 53 & 84 & 0.2 & - & -- & - & 420 & 4.3 & -- \\
\hline & 110 & 86 & 0.4 & - & - & - & 520 & 5.0 & - \\
\hline \multirow[t]{3}{*}{$6 S / 3 W-2 G 1$} & 130 & 200 & 0.5 & -- & 42 & -- & 740 & 6.8 & -- \\
\hline & 120 & 200 & 0.4 & - & - & -- & 790 & 7.5 & -- \\
\hline & - & -- & -- & - & - & -- & -- & -- & -- \\
\hline \multirow[t]{3}{*}{$6 S / 3 W-2 G 2$} & 200 & 270 & 0.4 & - & 50 & -- & 1,030 & 4.5 & - \\
\hline & 170 & 270 & 0.4 & - & - & - & 1,050 & 5.9 & -- \\
\hline & - & - & -- & -- & -- & -- & -- & -- & -- \\
\hline \multirow[t]{3}{*}{$6 \mathrm{~S} / 3 \mathrm{~W}-2 \mathrm{H} 2$} & 160 & 180 & 0.5 & -- & 50 & -- & 770 & 4.1 & -- \\
\hline & 160 & 190 & 0.4 & - & -- & -- & 840 & 3.4 & -- \\
\hline & -- & - & - & - & -- & -- & -- & - & -- \\
\hline \multirow[t]{3}{*}{$6 S / 3 W-3 C 1$} & 300 & 720 & 0.3 & -- & -- & 2,360 & -- & 9.9 & -- \\
\hline & 320 & 670 & 0.4 & -- & -- & - & 2,540 & 0.90 & -- \\
\hline & 260 & 500 & - & - & 71 & -- & 2,290 & - & -- \\
\hline \multirow[t]{2}{*}{$6 S / 3 W-3 C 2$} & 320 & 100 & 0.2 & -- & -- & -- & 710 & 0.54 & -- \\
\hline & 300 & 580 & 0.7 & - & -- & -- & 2,170 & 2.5 & - \\
\hline \multirow[t]{2}{*}{$6 \mathrm{~S} / 3 \mathrm{~W}-3 \mathrm{H} 2$} & -- & -- & - & -- & - & -- & 1,380 & - & -- \\
\hline & 200 & 390 & -- & - & -- & -- & 1,450 & -- & - \\
\hline $6 S / 3 W-3 L 1$ & 45 & 83 & -- & - & 63 & -- & - & 5.0 & $<0.010$ \\
\hline \multirow{2}{*}{$6 S / 3 W-4 K 1$} & 56 & 180 & - & - & -- & - & -- & 5.4 & - \\
\hline & 25 & 46 & 0.4 & -- & 18 & -- & 260 & 0.20 & - \\
\hline $6 S / 3 W-8 R 1$ & -- & - & - & -- & 40 & - & - & - & $<0.010$ \\
\hline $6 \mathrm{~S} / 3 \mathrm{~W}-9 \mathrm{E} 1$ & 39 & 87 & -- & -- & 39 & -- & -- & 11 & $<0.010$ \\
\hline $6 S / 3 W-9 G 1$ & 67 & 220 & -- & 0.2 & 35 & - & -- & 3.9 & $<0.010$ \\
\hline
\end{tabular}


Table 2. Historical water-quality data for selected wells in the Winchester, Menifee, and south Perris ground-water subbasins, California-Continued

\begin{tabular}{|c|c|c|c|c|c|c|c|c|c|}
\hline Well No. & $\begin{array}{c}\mathrm{NO}_{3}+\mathrm{NO}_{2} \\
\text { dissolved } \\
\text { (mg/L as } \mathrm{N} \text { ) }\end{array}$ & $\begin{array}{c}\text { Nitrogen } \\
\text { ammonia, } \\
\text { dissolved } \\
\text { (mg/L as } \mathrm{N} \text { ) }\end{array}$ & $\begin{array}{l}\text { Phosphorus, } \\
\text { dissolved } \\
\text { (mg/L) }\end{array}$ & $\begin{array}{l}\text { Phosphorus } \\
\text { ortho, } \\
\text { dissolved } \\
\text { (mg/L as } \mathrm{P} \text { ) }\end{array}$ & $\begin{array}{c}\text { Arsenic, } \\
\text { dissolved } \\
(\mu \mathrm{g} / \mathrm{L})\end{array}$ & $\begin{array}{l}\text { Barium, } \\
\text { dissolved } \\
(\mu g / L)\end{array}$ & $\begin{array}{c}\text { Beryllium, } \\
\text { dissolved } \\
(\mu \mathrm{g} / \mathrm{L})\end{array}$ & $\begin{array}{c}\text { Boron, } \\
\text { dissolved } \\
(\mu \mathrm{g} / \mathrm{L})\end{array}$ & $\begin{array}{c}\text { Cadmium, } \\
\text { dissolved } \\
(\mu \mathrm{g} / \mathrm{L})\end{array}$ \\
\hline & -- & -- & -- & -- & $<5$ & $<100$ & -- & -- & $<1$ \\
\hline & -- & -- & -- & - & $<5$ & 100 & -- & -- & $<1$ \\
\hline \multirow[t]{3}{*}{$6 \mathrm{~S} / 3 \mathrm{~W}-2 \mathrm{E} 1$} & - & -- & -- & -- & $<5$ & 100 & -- & -- & $<1$ \\
\hline & -- & -- & -- & -- & $<5$ & 200 & -- & -- & $<1$ \\
\hline & -- & -- & -- & -- & $<5$ & 200 & -- & -- & $<1$ \\
\hline \multirow[t]{4}{*}{$6 S / 3 W-2 F 1$} & -- & -- & -- & -- & -- & -- & -- & 130 & - \\
\hline & -- & -- & -- & -- & -- & -- & - & 50 & - \\
\hline & -- & -- & -- & -- & - & -- & -- & 220 & -- \\
\hline & -- & -- & - & -- & -- & -- & -- & 0 & -- \\
\hline \multirow[t]{3}{*}{$6 \mathrm{~S} / 3 \mathrm{~W}-2 \mathrm{G} 1$} & - & -- & -- & -- & -- & -- & -- & 200 & -- \\
\hline & -- & -- & -- & -- & -- & -- & -- & $<100$ & -- \\
\hline & -- & -. & -- & -- & 3.7 & -- & -- & - & 0.60 \\
\hline \multirow[t]{3}{*}{$6 S / 3 W-2 G 2$} & - & -- & - & - & - & -- & -- & 200 & - \\
\hline & -- & -- & -- & -- & -- & -- & -- & $<100$ & -- \\
\hline & -- & -- & -- & -- & 5.0 & -- & - & -- & 0.40 \\
\hline \multirow[t]{3}{*}{$6 \mathrm{~S} / 3 \mathrm{~W}-2 \mathrm{H} 2$} & -- & - & -- & -- & -- & -- & -- & 200 & -- \\
\hline & -- & -- & -- & -- & -- & -- & -- & $<100$ & -- \\
\hline & -- & -- & -- & -- & 2.9 & -- & $<0.2$ & - & 0.30 \\
\hline \multirow[t]{3}{*}{$6 S / 3 W-3 C 1$} & -- & $<1.0$ & -- & -- & -- & -- & -- & 600 & -- \\
\hline & -- & -- & - & -- & -- & -- & -- & & -- \\
\hline & -- & -- & -- & -- & -- & - & -- & -- & -- \\
\hline \multirow[t]{2}{*}{$6 S / 3 W-3 C 2$} & -- & 0.0 & -- & - & -- & -- & -- & 590 & -- \\
\hline & -- & -- & -- & -- & -- & -- & -- & 80 & -- \\
\hline \multirow[t]{2}{*}{$6 \mathrm{~S} / 3 \mathrm{~W}-3 \mathrm{H} 2$} & - & -- & -- & -- & -- & -- & - & -- & -- \\
\hline & -- & -- & -- & -- & -- & -- & - & - & -- \\
\hline $6 S / 3 W-3 L 1$ & 5.0 & 0.03 & -- & 0.03 & 8.0 & 86 & $<0.5$ & 50 & $<1$ \\
\hline \multirow[t]{2}{*}{$6 \mathrm{~S} / 3 \mathrm{~W}-4 \mathrm{~K} 1$} & -- & -- & -- & -- & -- & - & - & 60 & -- \\
\hline & -- & -- & -- & -- & -- & -- & -- & 100 & -- \\
\hline $6 S / 3 W-8 R 1$ & 8.8 & $<0.01$ & -- & 0.07 & 2.0 & 360 & $<0.5$ & 110 & -- \\
\hline $6 \mathrm{~S} / 3 W-9 \mathrm{E} 1$ & 11 & $<0.01$ & -- & 0.31 & 3.0 & 100 & $<0.5$ & 130 & -- \\
\hline $6 S / 3 W-9 G 1$ & 3.9 & 0.02 & - & 0.05 & 3.0 & 130 & $<0.5$ & 100 & -- \\
\hline
\end{tabular}


Table 2. Historical water-quality data for selected wells in the Winchester, Menifee, and south Perris ground-water subbasins, California-Continued

\begin{tabular}{|c|c|c|c|c|c|c|c|c|c|}
\hline Well No. & $\begin{array}{c}\text { Chromium, } \\
\text { dissolved } \\
(\mu g / L)\end{array}$ & $\begin{array}{c}\text { Cobalt, } \\
\text { dissolved } \\
(\mu \mathrm{g} / \mathrm{L})\end{array}$ & $\begin{array}{c}\text { Copper, } \\
\text { dissolved } \\
(\mu g / L)\end{array}$ & $\begin{array}{c}\text { Iron, } \\
\text { dissolved } \\
(\mu \mathrm{g} / \mathrm{L})\end{array}$ & $\begin{array}{c}\text { Lead, } \\
\text { dissolved } \\
(\mu g / L)\end{array}$ & $\begin{array}{l}\text { Lithium, } \\
\text { dissolved } \\
(\mu \mathrm{g} / \mathrm{L})\end{array}$ & $\begin{array}{c}\text { Manganese, } \\
\text { dissolved } \\
(\mu g / L)\end{array}$ & $\begin{array}{l}\text { Molybdenum, } \\
\text { dissolved } \\
(\mu \mathrm{g} / \mathrm{L})\end{array}$ & $\begin{array}{c}\text { Nickel, } \\
\text { dissolved } \\
(\mu \mathrm{g} / \mathrm{L})\end{array}$ \\
\hline & $<10$ & -- & $<10$ & 2,300 & $<5$ & - & 900 & - & -- \\
\hline & $<10$ & -- & $<10$ & $<20$ & $<5$ & -- & 340 & -- & -- \\
\hline \multirow[t]{3}{*}{$6 \mathrm{~S} / 3 \mathrm{~W}-2 \mathrm{E} 1$} & $<10$ & - & 40 & 80 & $<5$ & - & 370 & -- & - \\
\hline & $<10$ & - & $<10$ & 150 & 7.0 & - & 470 & - & -- \\
\hline & $<10$ & -- & 30 & $<20$ & $<5$ & - & 220 & -- & -- \\
\hline \multirow[t]{4}{*}{$6 \mathrm{~S} / 3 \mathrm{~W}-2 \mathrm{~F} 1$} & -- & -- & - & -- & -- & - & - & -- & -- \\
\hline & - & -- & -- & - & -- & -- & - & -- & - \\
\hline & - & - & -- & - & -- & -- & - & - & -- \\
\hline & - & - & -- & -- & - & -- & -- & - & -- \\
\hline \multirow[t]{3}{*}{$6 S / 3 W-2 G 1$} & -- & - & -- & -- & -- & -- & -- & -- & - \\
\hline & -- & - & - & - & -- & -- & -- & -- & -- \\
\hline & 2.0 & -- & 3.8 & - & 2.8 & -- & -- & - & 3.0 \\
\hline \multirow[t]{3}{*}{$6 S / 3 W-2 G 2$} & -- & -- & -- & -- & -- & -- & -- & -- & -- \\
\hline & - & -- & -- & -- & -- & -- & -- & - & - \\
\hline & 4.0 & - & 3.0 & - & 2.4 & - & -. & -- & $<4$ \\
\hline \multirow[t]{3}{*}{$6 \mathrm{~S} / 3 \mathrm{~W}-2 \mathrm{H} 2$} & - & - & -- & -- & - & - & -- & -- & - \\
\hline & -- & -- & -- & -- & -- & -- & - & - & - \\
\hline & 2.0 & - & 4.8 & -- & 2.1 & -- & -- & -- & $<3$ \\
\hline \multirow[t]{3}{*}{$6 S / 3 W-3 C 1$} & -- & - & -- & - & -- & -- & - & -- & -- \\
\hline & - & - & -- & 320 & - & -- & 540 & - & -- \\
\hline & -- & -- & 100 & 3,000 & -- & - & 1,500 & -- & - \\
\hline \multirow[t]{2}{*}{$6 S / 3 W-3 C 2$} & -- & - & -- & -- & -- & - & -- & -- & -- \\
\hline & - & - & -- & - & -- & -- & - & -- & - \\
\hline \multirow[t]{2}{*}{$6 \mathrm{~S} / 3 \mathrm{~W}-3 \mathrm{H} 2$} & -- & -- & -- & -- & - & -- & -- & -- & - \\
\hline & -- & -- & 170 & 3,500 & -- & -- & 150 & -- & - \\
\hline $6 \mathrm{~S} / 3 \mathrm{~W}-3 \mathrm{~L} 1$ & $<5$ & $<3$ & $<10$ & 5 & $<10$ & 20 & $<1$ & $<10$ & $<10$ \\
\hline \multirow[t]{2}{*}{$6 S / 3 W-4 K 1$} & -- & -- & - & -- & - & - & - & -- & - \\
\hline & - & - & - & - & - & - & - & - & - \\
\hline $6 \mathrm{~S} / 3 \mathrm{~W}-8 \mathrm{R} 1$ & $<5$ & $<3$ & $<10$ & $<3$ & $<10$ & 40 & 2 & $<10$ & $<10$ \\
\hline $6 \mathrm{~S} / 3 \mathrm{~W}-9 \mathrm{E} 1$ & $<5$ & $<3$ & $<10$ & $<3$ & $<10$ & 40 & $<1$ & $<10$ & $<10$ \\
\hline $6 \mathrm{~S} / 3 W-9 \mathrm{G} 1$ & $<5$ & $<3$ & $<10$ & $<3$ & $<10$ & 30 & $<1$ & $<10$ & $<10$ \\
\hline
\end{tabular}


Table 2. Historical water-quality data for selected wells in the Winchester, Menifee, and south Perris ground-water subbasins, California--Continued

\begin{tabular}{|c|c|c|c|c|c|c|}
\hline Well No. & $\begin{array}{c}\text { Selenium, } \\
\text { dissolved } \\
(\mu g / L)\end{array}$ & $\begin{array}{c}\text { Silver, } \\
\text { dissolved } \\
(\mu g / L)\end{array}$ & $\begin{array}{l}\text { Strontium, } \\
\text { dissolved } \\
(\mu \mathrm{g} / \mathrm{L})\end{array}$ & $\begin{array}{l}\text { Vanadium, } \\
\text { dissolved } \\
(\mu \mathrm{g} / \mathrm{L})\end{array}$ & $\begin{array}{c}\text { Zinc, } \\
\text { dissolved } \\
(\mu \mathrm{g} / \mathrm{L})\end{array}$ & Source of data \\
\hline & $<5$ & $<10$ & - & -- & 230 & Babcock - $375^{\prime}-395^{\prime}$ \\
\hline & $<5$ & $<10$ & -- & -- & $<10$ & Babcock - 555'-575 \\
\hline \multirow[t]{3}{*}{$6 S / 3 W-2 E 1$} & $<5$ & $<10$ & -- & -- & 330 & Babcock - Composite \\
\hline & $<5$ & $<10$ & -- & -- & $<10$ & Babcock - 370'-390' \\
\hline & $<5$ & $<10$ & - & -- & $<10$ & Babcock - 440'-460' \\
\hline \multirow[t]{4}{*}{$6 \mathrm{~S} / 3 \mathrm{~W}-2 \mathrm{~F} 1$} & -. & -- & - & -- & -- & RCFCD \\
\hline & - & - & -- & -- & - & RCFCD \\
\hline & -. & -- & -- & -- & - & RCFCD \\
\hline & - & - & - & - & - & RCFCD \\
\hline \multirow[t]{3}{*}{$6 S / 3 W-2 G 1$} & - & -- & - & -- & -- & EMWD/Babcock \\
\hline & -- & -- & -- & - & -- & Babcock \\
\hline & 5.0 & $<0.1$ & -- & -- & 5 & EMWD \\
\hline \multirow[t]{3}{*}{$6 S / 3 W-2 G 2$} & -- & -- & -- & -- & -- & EMWD/Babcock \\
\hline & -- & -- & -- & -- & -- & Babcock \\
\hline & 8.0 & $<0.1$ & -- & -- & 8 & EMWD \\
\hline \multirow[t]{3}{*}{$6 \mathrm{~S} / 3 \mathrm{~W}-2 \mathrm{H} 2$} & -- & -- & -- & -- & -- & EMWD/Babcock \\
\hline & - & - & -- & -- & - & Babcock \\
\hline & 9.0 & $<0.1$ & -- & -. & 6 & EMWD \\
\hline \multirow[t]{3}{*}{$6 S / 3 W-3 C 1$} & -- & - & -- & -- & -- & EMWD \\
\hline & -- & -- & -- & -- & -- & Babcock \\
\hline & - & - & -- & -- & 170 & Babcock \\
\hline \multirow[t]{2}{*}{$6 S / 3 W-3 C 2$} & -. & -- & -- & -. & - & EMWD \\
\hline & -- & -- & - & -- & -- & DWR \\
\hline \multirow[t]{2}{*}{$6 S / 3 W-3 H 2$} & -- & -- & - & -- & -- & EMWD \\
\hline & -- & -- & -- & -. & 60 & Babcock \\
\hline $6 S / 3 W-3 L 1$ & 3.0 & $<1.0$ & 350 & 21 & 280 & USGS \\
\hline \multirow[t]{2}{*}{$6 S / 3 W-4 K 1$} & -- & - & -- & -- & - & RCFCD \\
\hline & -- & - & - & -- & - & RCFCD \\
\hline $6 \mathrm{~S} / 3 \mathrm{~W}-8 \mathrm{R} 1$ & 2.0 & $<1.0$ & 300 & 17 & 5 & USGS \\
\hline $6 \mathrm{~S} / 3 W-9 E 1$ & 1.0 & $<1.0$ & 280 & 26 & 4 & USGS \\
\hline $6 S / 3 W-9 G 1$ & 4.0 & $<1.0$ & 430 & 33 & 310 & USGS \\
\hline
\end{tabular}


Table 3. Water-level data for selected wells in the Winchester, Menifee, and south Perris ground-water subbasins, California

[ft, foot; $\mathrm{ft}$ blw LSD, feet below land surface datum; R, number is a reported value; --, no data. Altitude, in feet above sea level. Asterisk $\left({ }^{\star}\right)$ indicates that altitude and water levels are from a reference point other than LSD. Two asterisks ( $\left.{ }^{\star \star}\right)$ indicate the old RCFCD well no. Source of data: DWR, California Department of Water Resources; MWD, Metropolitan Water District of Southern California; RCFCD; Riverside County Flood Control and Water Conservation District; EMWD, Eastern Municipal Water District. Wells not shown in figure 1 were not located in the field or have been destroyed. Additional data from non-USGS sources are available]

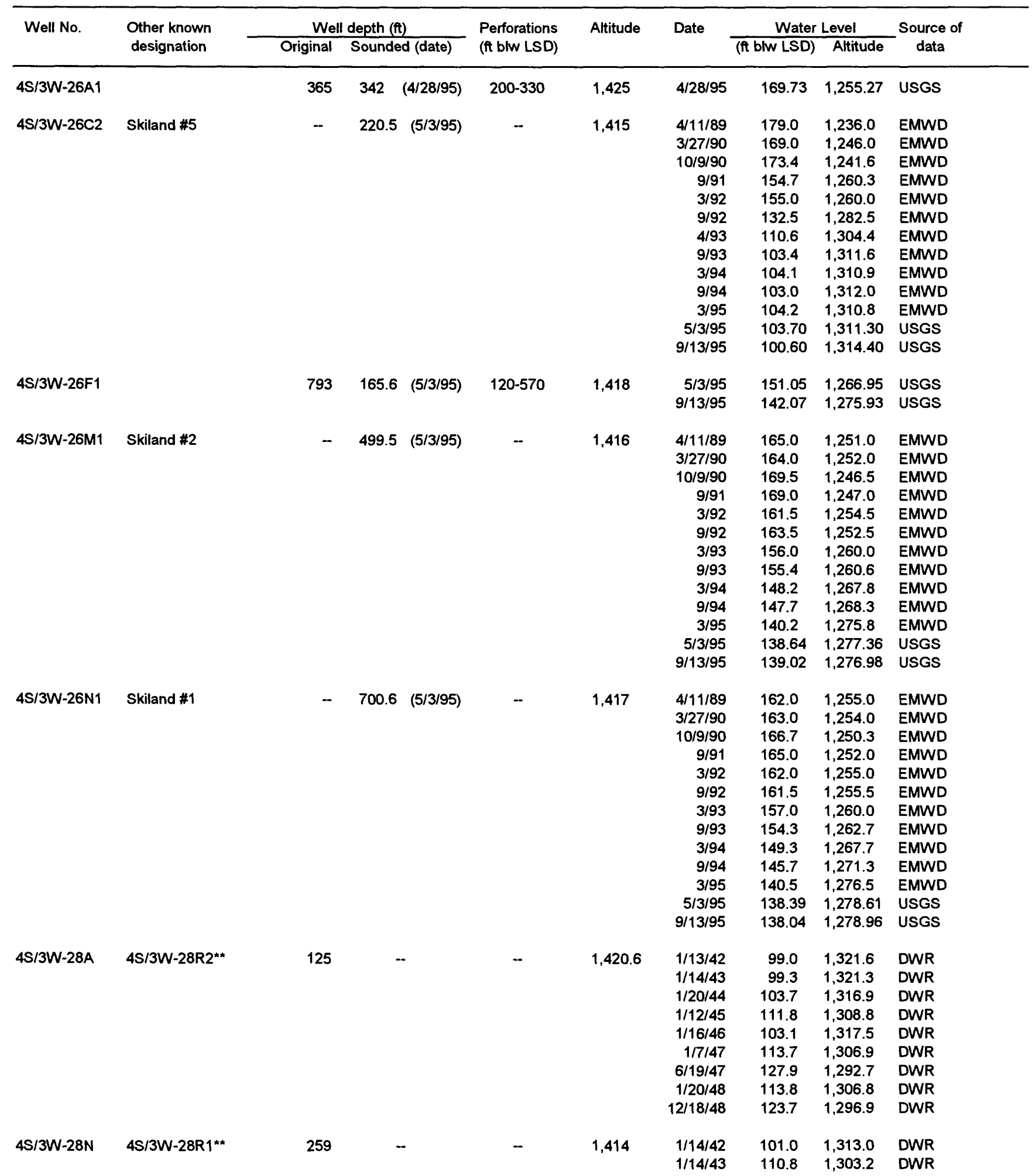


Table 3. Water-level data for selected wells in the Winchester, Menifee, and south Perris ground-water subbasins, California-Continued

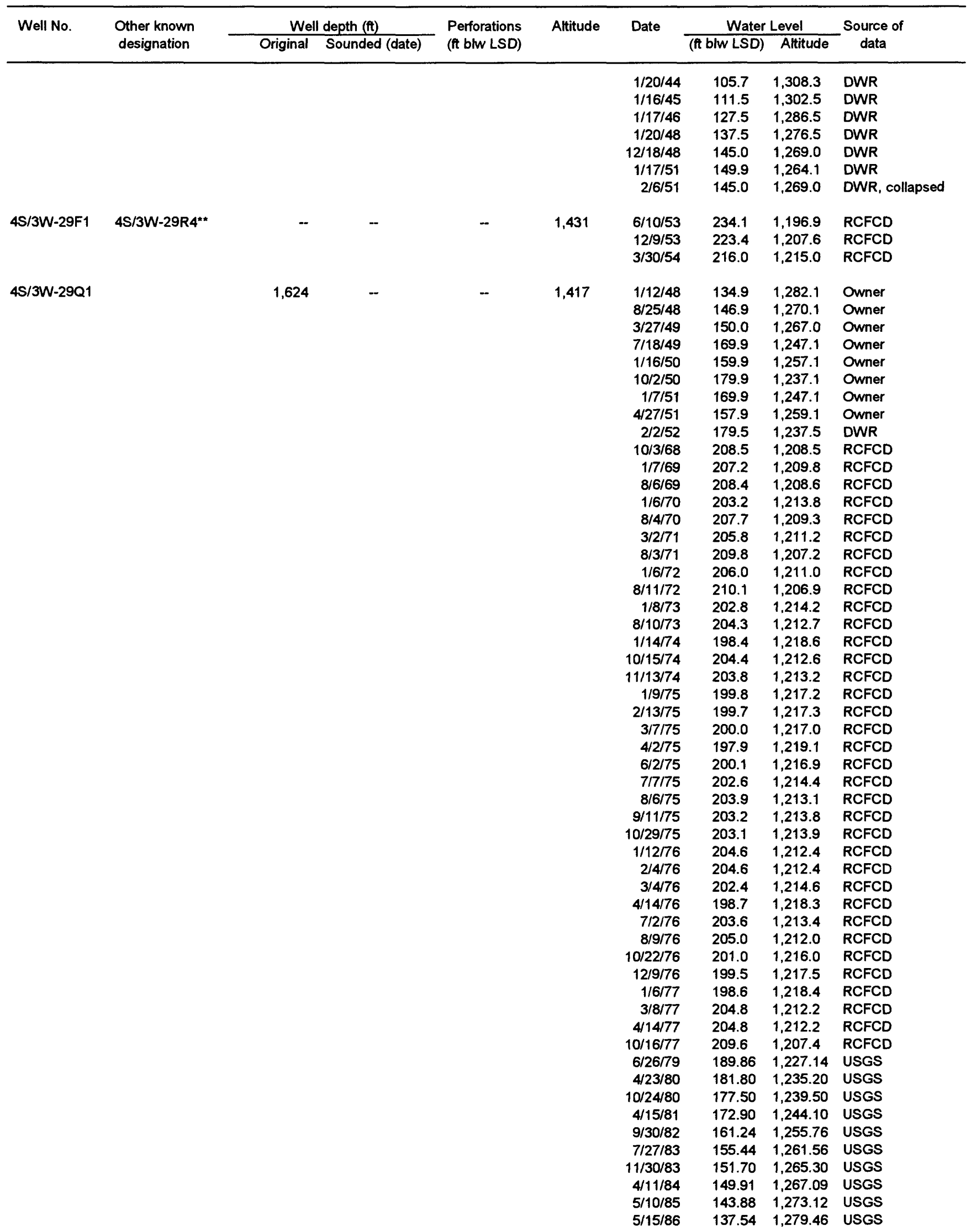


Table 3. Water-level data for selected wells in the Winchester, Menifee, and south Perris ground-water subbasins, CaliforniaContinued

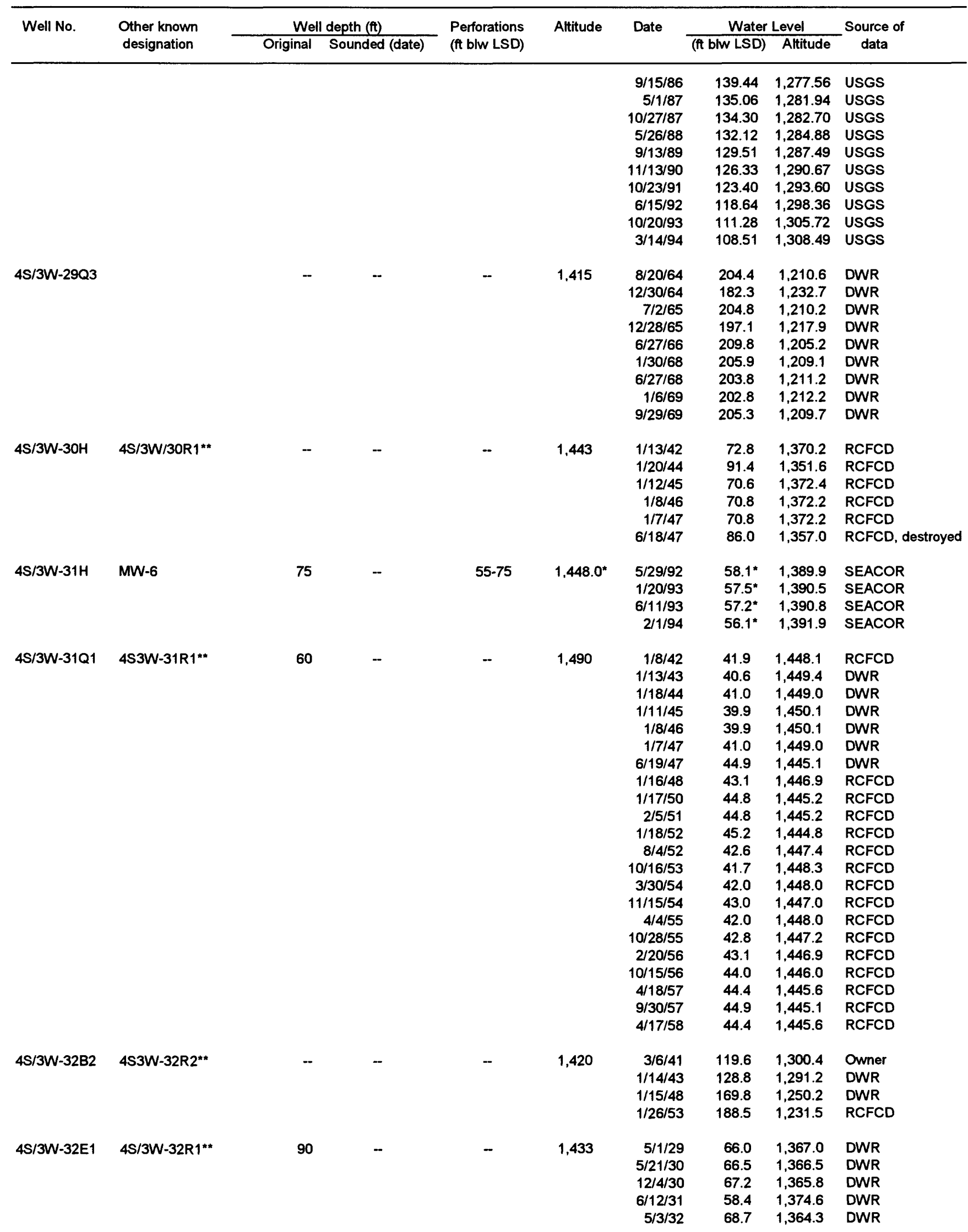


Table 3. Water-level data for selected wells in the Winchester, Menifee, and south Perris ground-water subbasins, CaliforniaContinued

\begin{tabular}{|c|c|c|c|c|c|c|c|c|}
\hline \multirow[t]{2}{*}{ Well No. } & \multirow{2}{*}{$\begin{array}{l}\text { Other known } \\
\text { designation }\end{array}$} & Well depth (tt) & \multirow{2}{*}{$\begin{array}{l}\text { Perforations } \\
\text { (ft blw LSD) }\end{array}$} & \multirow[t]{2}{*}{ Altitude } & \multirow[t]{2}{*}{ Date } & \multicolumn{2}{|c|}{ Water Level } & \multirow{2}{*}{$\begin{array}{c}\text { Source of } \\
\text { data }\end{array}$} \\
\hline & & Original Sounded (date) & & & & (ft blw LSD) & Altitude & \\
\hline & & & & & $11 / 18 / 32$ & 69.3 & $1,363.7$ & DWR \\
\hline & & & & & $5 / 18 / 33$ & 69.7 & $1,363.3$ & DWR \\
\hline & & & & & $11 / 10 / 33$ & 69.9 & $1,363.1$ & DWR \\
\hline & & & & & $2 / 6 / 34$ & 69.9 & $1,363.1$ & DWR \\
\hline & & & & & $12 / 4 / 34$ & 70.5 & $1,362.5$ & DWR \\
\hline & & & & & $5 / 7 / 35$ & 70.8 & $1,362.2$ & DWR \\
\hline & & & & & $11 / 5 / 35$ & 71.0 & $1,362.0$ & DWR \\
\hline & & & & & $7 / 30 / 36$ & 71.2 & $1,361.8$ & DWR \\
\hline & & & & & $12 / 22 / 36$ & 70.0 & $1,363.0$ & DWR \\
\hline & & & & & $6 / 21 / 37$ & 70.2 & $1,362.8$ & DWR \\
\hline & & & & & $11 / 19 / 37$ & 70.1 & $1,362.9$ & DWR \\
\hline & & & & & $5 / 4 / 38$ & 70.3 & $1,362.7$ & DWR \\
\hline & & & & & $11 / 3 / 38$ & 70.6 & $1,362.4$ & DWR \\
\hline & & & & & 6/9/39 & 71.0 & $1,362.0$ & DWR \\
\hline & & & & & $11 / 15 / 39$ & 71.0 & $1,362.0$ & DWR \\
\hline & & & & & $8 / 23 / 40$ & 71.5 & $1,361.5$ & DWR \\
\hline & & & & & $1 / 15 / 41$ & 71.4 & $1,361.6$ & DWR \\
\hline & & & & & $8 / 20 / 41$ & 72.0 & $1,361.0$ & DWR \\
\hline & & & & & $3 / 31 / 42$ & 74.1 & $1,358.9$ & DWR \\
\hline & & & & & $1 / 20 / 43$ & 68.8 & $1,364.2$ & DWR \\
\hline & & & & & $1 / 12 / 45$ & 66.4 & $1,366.6$ & DWR \\
\hline & & & & & $7 / 17 / 45$ & 65.5 & $1,367.5$ & DWR \\
\hline & & & & & $1 / 9 / 46$ & 70.1 & $1,362.9$ & DWR \\
\hline & & & & & $6 / 25 / 46$ & 63.5 & $1,369.5$ & DWR \\
\hline & & & & & $1 / 7 / 47$ & 74.7 & $1,358.3$ & DWR \\
\hline & & & & & $6 / 19 / 47$ & 82.4 & $1,350.6$ & RCFCD \\
\hline & & & & & $3 / 12 / 48$ & 62.7 & $1,370.3$ & RCFCD \\
\hline & & & & & $7 / 12 / 48$ & 62.7 & $1,370.3$ & RCFCD \\
\hline & & & & & $1 / 11 / 49$ & 65.5 & $1,367.5$ & RCFCD \\
\hline & & & & & $8 / 9 / 49$ & 63.7 & $1,369.3$ & RCFCD \\
\hline & & & & & $1 / 17 / 50$ & 63.9 & $1,369.1$ & RCFCD \\
\hline & & & & & $7 / 5 / 50$ & 65.1 & $1,367.9$ & RCFCD \\
\hline & & & & & $1 / 11 / 51$ & 65.1 & $1,367.9$ & RCFCD \\
\hline & & & & & $7 / 27 / 51$ & 66.1 & $1,366.9$ & RCFCD \\
\hline & & & & & $1 / 15 / 52$ & 68.9 & $1,364.1$ & RCFCD \\
\hline & & & & & $8 / 4 / 52$ & 68.7 & $1,364.3$ & RCFCD \\
\hline & & & & & $2 / 26 / 53$ & 67.5 & $1,365.5$ & RCFCD \\
\hline & & & & & $12 / 22 / 53$ & 68.8 & $1,364.2$ & RCFCD \\
\hline & & & & & $6 / 1 / 54$ & 69.6 & $1,363.4$ & RCFCD \\
\hline & & & & & $11 / 5 / 54$ & 69.9 & $1,363.1$ & RCFCD \\
\hline & & & & & $7 / 7 / 55$ & 70.6 & $1,362.4$ & RCFCD \\
\hline & & & & & $12 / 6 / 55$ & 71.1 & $1,361.9$ & RCFCD \\
\hline & & & & & $7 / 31 / 56$ & 71.6 & $1,361.4$ & RCFCD \\
\hline & & & & & $12 / 4 / 56$ & 71.8 & $1,361.2$ & RCFCD \\
\hline & & & & & $7 / 2 / 57$ & 73.4 & $1,359.6$ & RCFCD \\
\hline & & & & & $12 / 5 / 57$ & 72.1 & $1,360.9$ & RCFCD \\
\hline & & & & & $8 / 11 / 58$ & 72.4 & $1,360.6$ & RCFCD \\
\hline & & & & & $12 / 18 / 58$ & 73.2 & $1,359.8$ & RCFCD \\
\hline & & & & & $7 / 7 / 59$ & 72.6 & $1,360.5$ & RCFCD \\
\hline & & & & & $12 / 2 / 59$ & 77.6 & $1,355.4$ & RCFCD \\
\hline & & & & & $8 / 4 / 60$ & 80.8 & $1,352.2$ & RCFCD \\
\hline & & & & & $12 / 6 / 60$ & 80.3 & $1,352.7$ & RCFCD \\
\hline & & & & & $7 / 11 / 61$ & 74.5 & $1,358.5$ & RCFCD \\
\hline & & & & & $12 / 28 / 61$ & 72.4 & $1,360.6$ & RCFCD \\
\hline & & & & & $8 / 6 / 62$ & 73.9 & $1,359.1$ & RCFCD \\
\hline & & & & & $12 / 5 / 62$ & 73.9 & $1,359.1$ & RCFCD \\
\hline & & & & & $8 / 6 / 63$ & 74.6 & $1,358.4$ & RCFCD \\
\hline & & & & & $12 / 4 / 63$ & 74.6 & $1,358.4$ & RCFCD \\
\hline & & & & & $6 / 8 / 64$ & 74.7 & $1,358.3$ & RCFCD \\
\hline & & & & & $12 / 23 / 64$ & 75.4 & $1,357.6$ & RCFCD \\
\hline & & & & & $7 / 13 / 65$ & 75.7 & $1,357.3$ & RCFCD \\
\hline & & & & & $12 / 20 / 65$ & 75.8 & $1,357.2$ & RCFCD \\
\hline & & & & & $7 / 7 / 66$ & 76.2 & $1,356.8$ & RCFCD \\
\hline & & & & & $12 / 13 / 66$ & 75.9 & $1,357.1$ & RCFCD \\
\hline & & & & & $5 / 2 / 67$ & 75.7 & $1,357.3$ & RCFCD \\
\hline
\end{tabular}


Table 3. Water-level data for selected wells in the Winchester, Menifee, and south Perris ground-water subbasins, California-Continued

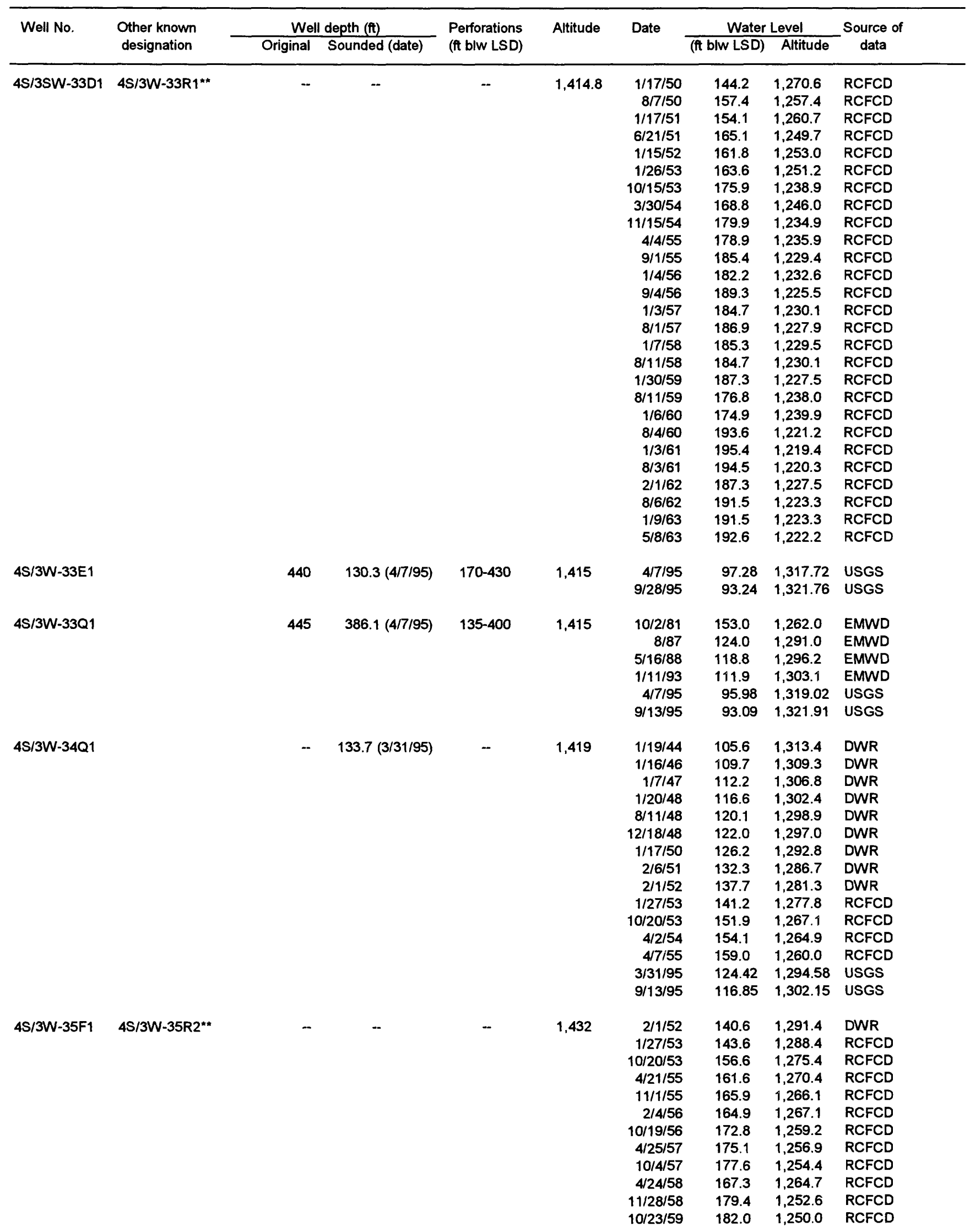


Table 3. Water-level data for selected wells in the Winchester, Menifee, and south Perris ground-water subbasins, CaliforniaContinued

\begin{tabular}{|c|c|c|c|c|c|c|c|c|c|}
\hline \multirow[t]{2}{*}{ Well No. } & \multirow{2}{*}{$\begin{array}{l}\text { Other known } \\
\text { designation }\end{array}$} & \multicolumn{2}{|c|}{ Well depth (ft) } & \multirow{2}{*}{$\begin{array}{l}\text { Perforations } \\
\text { (ft blw LSD) }\end{array}$} & \multirow[t]{2}{*}{ Altitude } & \multirow[t]{2}{*}{ Date } & \multicolumn{2}{|c|}{ Water Level } & \multirow{2}{*}{$\begin{array}{c}\text { Source of } \\
\text { data }\end{array}$} \\
\hline & & Original & Sounded (date) & & & & (ft blw LSD) & Altitude & \\
\hline & & & & & & $3 / 18 / 60$ & 178.4 & $1,253.6$ & RCFCD \\
\hline & & & & & & $10 / 11 / 60$ & 183.7 & $1,248.3$ & RCFCD \\
\hline & & & & & & $3 / 23 / 61$ & 183.5 & $1,248.5$ & RCFCD \\
\hline & & & & & & $11 / 2 / 61$ & 212.5 & $1,219.5$ & RCFCD \\
\hline & & & & & & $3 / 21 / 62$ & 196.7 & 1.235 .3 & RCFCD \\
\hline & & & & & & $10 / 16 / 62$ & 212.0 & $1,220.0$ & RCFCD \\
\hline & & & & & & $3 / 26 / 63$ & 212.1 & $1,219.9$ & RCFCD \\
\hline & & & & & & $10 / 21 / 63$ & 213.1 & $1,218.9$ & RCFCD \\
\hline & & & & & & $3 / 24 / 64$ & 213.8 & $1,218.2$ & RCFCD \\
\hline & & & & & & $10 / 2 / 64$ & 211.2 & $1,220.8$ & RCFCD \\
\hline & & & & & & $4 / 15 / 65$ & 212.4 & $1,219.6$ & RCFCD \\
\hline & & & & & & $11 / 3 / 65$ & 207.1 & $1,224.9$ & RCFCD \\
\hline & & & & & & $10 / 25 / 66$ & 222.6 & $1,209.4$ & RCFCD \\
\hline & & & & & & $10 / 26 / 67$ & 220.4 & $1,211.6$ & RCFCD \\
\hline & & & & & & $11 / 8 / 68$ & 223.7 & $1,208.3$ & RCFCD \\
\hline & & & & & & $4 / 7 / 69$ & 209.9 & $1,222.1$ & RCFCD \\
\hline & & & & & & $11 / 6 / 69$ & 221.2 & 1.210 .8 & RCFCD \\
\hline & & & & & & $4 / 8 / 70$ & 218.4 & $1,213.6$ & RCFCD \\
\hline & & & & & & $10 / 8 / 70$ & 210.8 & $1,221.2$ & RCFCD \\
\hline & & & & & & $4 / 6 / 71$ & 217.8 & $1,214.2$ & RCFCD \\
\hline & & & & & & $10 / 14 / 71$ & 208.5 & $1,223.5$ & RCFCD \\
\hline \multirow[t]{10}{*}{$5 S / 2 W-19 N 1$} & & 358 & $290.5(5 / 3 / 95)$ & $96-312$ & 1,459 & $9 / 18 / 87$ & 29.40 & 1.429 .60 & USGS \\
\hline & & & & & & $3 / 18 / 92$ & 37.79 & $1,421.21$ & USGS \\
\hline & & & & & & $9 / 3 / 92$ & 42.47 & $1,416.53$ & USGS \\
\hline & & & & & & $12 / 29 / 92$ & 40.03 & $1,418.97$ & USGS \\
\hline & & & & & & $5 / 13 / 93$ & 37.53 & $1,421.47$ & USGS \\
\hline & & & & & & $9 / 10 / 93$ & 34.34 & $1,424.66$ & USGS \\
\hline & & & & & & $12 / 29 / 93$ & 40.03 & $1,418.97$ & USGS \\
\hline & & & & & & $2 / 10 / 94$ & 33.70 & $1,425.30$ & USGS \\
\hline & & & & & & $3 / 15 / 94$ & 33.25 & $1,425.75$ & USGS \\
\hline & & & & & & $11 / 18 / 94$ & 32.77 & $1,426.23$ & USGS \\
\hline \multirow[t]{4}{*}{$5 S / 2 W-19 N 2$} & & 373 & $360.35(5 / 16 / 95)$ & $127-141$ & 1,458 & $5 / 16 / 95$ & 33.43 & $1,424.57$ & USGS \\
\hline & & & & $157-217$ & & & & & \\
\hline & & & & $231-251$ & & & & & \\
\hline & & & & $265-365$ & & & & & \\
\hline \multirow[t]{3}{*}{$5 S / 2 W-27 N 1$} & & 105 & $58(5 / 13 / 93)$ & $40-105$ & 1,491 & $5 / 12 / 93$ & 9.27 & $1,481.73$ & USGS \\
\hline & & & & & & $12 / 30 / 93$ & 11.27 & $1,479.73$ & USGS \\
\hline & & & & & & $6 / 16 / 94$ & 8.59 & $1,482.41$ & USGS \\
\hline $5 S / 2 W-28 C 1$ & & 300 & - & no casing & 1,475 & $5 / 22 / 95$ & 22.68 & $1,452.32$ & USGS \\
\hline 5S/2W-28D1 & & - & $154.9(5 / 16 / 95)$ & - & 1,463 & $5 / 16 / 95$ & 10.98 & $1,452.02$ & USGS \\
\hline \multirow[t]{5}{*}{$5 S / 2 W-28 E 1$} & & 455 & $400.3(12 / 3 / 93)$ & $395-400$ & 1,459 & $12 / 3 / 93$ & 21.20 & $1,437.80$ & USGS \\
\hline & & & & & & $2 / 10 / 94$ & 9.53 & $1,449.47$ & USGS \\
\hline & & & & & & $6 / 16 / 94$ & 9.38 & $1,449.62$ & USGS \\
\hline & & & & & & $6 / 21 / 94$ & 9.19 & 1.449 .81 & USGS \\
\hline & & & & & & $11 / 17 / 94$ & 9.54 & $1,449.46$ & USGS \\
\hline \multirow[t]{5}{*}{$5 S / 2 W-28 E 2$} & & 455 & $311.5(12 / 3 / 93)$ & $306-311$ & 1,459 & $12 / 3 / 93$ & 7.08 & $1,451.92$ & USGS \\
\hline & & & & & & $2 / 10 / 94$ & 6.87 & $1,452.13$ & USGS \\
\hline & & & & & & $6 / 16 / 94$ & 7.68 & $1,451.32$ & USGS \\
\hline & & & & & & $6 / 21 / 94$ & 7.66 & $1,451.34$ & USGS \\
\hline & & & & & & $11 / 17 / 94$ & 8.45 & $1,450.55$ & USGS \\
\hline \multirow[t]{5}{*}{$5 S / 2 W-28 E 3$} & & 455 & $233.8(12 / 3 / 93)$ & $228-233$ & 1,459 & $12 / 3 / 93$ & 25.67 & $1,433.33$ & USGS \\
\hline & & & & & & $2 / 10 / 94$ & 9.94 & $1,449.06$ & USGS \\
\hline & & & & & & $6 / 16 / 94$ & 10.39 & $1,448.61$ & USGS \\
\hline & & & & & & $6 / 21 / 94$ & 10.50 & $1,448.50$ & USGS \\
\hline & & & & & & $11 / 17 / 94$ & 7.83 & $1,451.17$ & USGS \\
\hline \multirow[t]{2}{*}{$5 S / 2 W-29 J 1$} & & 100 & $62.5(6 / 2 / 95)$ & $40-60$ & 1,455 & $6 / 2 / 95$ & 5.17 & $1,449.83$ & USGS \\
\hline & & & & & & $9 / 13 / 95$ & 8.49 & $1,446.51$ & USGS \\
\hline
\end{tabular}


Table 3. Water-level data for selected wells in the Winchester, Menifee, and south Perris ground-water subbasins, California-Continued

\begin{tabular}{|c|c|c|c|c|c|c|c|c|c|}
\hline \multirow[t]{2}{*}{ Well No. } & \multirow{2}{*}{$\begin{array}{l}\text { Other known } \\
\text { designation }\end{array}$} & \multicolumn{2}{|c|}{ Well depth (ft) } & \multirow{2}{*}{$\begin{array}{l}\text { Perforations } \\
\text { (ft blw LSD) }\end{array}$} & \multirow[t]{2}{*}{ Altitude } & \multirow[t]{2}{*}{ Date } & \multicolumn{2}{|c|}{ Water Level } & \multirow{2}{*}{$\begin{array}{c}\text { Source of } \\
\text { data }\end{array}$} \\
\hline & & Original & Sounded (date) & & & & (ft blw LSD) & Altitude & \\
\hline $5 S / 2 W-29 L 2$ & & & 85 R (7/3/94) & -- & 1,458 & $5 / 16 / 95$ & 13.60 & $1,444.40$ & USGS \\
\hline $5 S / 2 W-29 L 3$ & & 200 & 12 & $\begin{array}{l}50-70,100- \\
0,160-180\end{array}$ & 1,455 & $\begin{array}{l}5 / 15 / 95 \\
8 / 14 / 95\end{array}$ & $\begin{array}{l}7.86 \\
9.85\end{array}$ & $\begin{array}{l}1,447.14 \\
1,445.15\end{array}$ & $\begin{array}{l}\text { USGS } \\
\text { USGS }\end{array}$ \\
\hline $5 S / 2 W-29 N 1$ & & - & $113.5(12 / 30 / 92)$ & - & 1,450 & $\begin{array}{r}12 / 30 / 92 \\
5 / 13 / 93 \\
2 / 10 / 94 \\
11 / 17 / 94 \\
6 / 23 / 95 \\
7 / 12 / 95 \\
9 / 13 / 95\end{array}$ & $\begin{array}{r}15.02 \\
5.64 \\
8.00 \\
8.57 \\
6.39 \\
6.83 \\
8.16\end{array}$ & $\begin{array}{l}1,434.98 \\
1,444.36 \\
1,442.00 \\
1,441.43 \\
1,443.61 \\
1,443.17 \\
1,441.84\end{array}$ & $\begin{array}{l}\text { USGS } \\
\text { USGS } \\
\text { USGS } \\
\text { USGS } \\
\text { USGS } \\
\text { USGS } \\
\text { USGS }\end{array}$ \\
\hline $5 S / 2 W-30 A 1$ & Winchester \#5 & 70 & $71.2(5 / 23 / 95)$ & $50-70$ & 1,475 & $\begin{array}{r}12 / 93 \\
3 / 94 \\
9 / 94 \\
3 / 95 \\
5 / 23 / 95 \\
6 / 95 \\
7 / 6 / 95 \\
9 / 13 / 95\end{array}$ & $\begin{array}{l}29.2 \\
28.6 \\
29.7 \\
19.3 \\
18.79 \\
20.8 \\
23.08 \\
30.35\end{array}$ & $\begin{array}{l}1,445.8 \\
1,446.4 \\
1,445.3 \\
1,455.7 \\
1,456.21 \\
1,454.2 \\
1,451.92 \\
1,444.65\end{array}$ & $\begin{array}{l}\text { EMWD } \\
\text { EMWD } \\
\text { EMWD } \\
\text { EMWD } \\
\text { USGS } \\
\text { EMWD } \\
\text { USGS } \\
\text { USGS }\end{array}$ \\
\hline $5 S / 2 W-30 B 1$ & Winchester \#4 & 70 & $70.4(5 / 23 / 95)$ & $50-70$ & 1,468 & $\begin{array}{r}12 / 93 \\
3 / 94 \\
9 / 94 \\
3 / 95 \\
5 / 23 / 95 \\
7 / 6 / 95 \\
9 / 13 / 95\end{array}$ & $\begin{array}{l}24.7 \\
21.8 \\
20.9 \\
15.6 \\
14.63 \\
16.24 \\
20.92\end{array}$ & $\begin{array}{l}1,443.3 \\
1,446.2 \\
1,447.1 \\
1,452.4 \\
1,453.37 \\
1,451.76 \\
1,447.08\end{array}$ & $\begin{array}{l}\text { EMWD } \\
\text { EMWD } \\
\text { EMWD } \\
\text { EMWD } \\
\text { USGS } \\
\text { USGS } \\
\text { USGS }\end{array}$ \\
\hline $5 S / 2 W-30 B 2$ & Winchester \#3 & 70 & $70.7(6 / 2 / 95)$ & $50-70$ & 1,457 & $\begin{array}{r}12 / 93 \\
3 / 94 \\
9 / 94 \\
3 / 95 \\
6 / 95 \\
6 / 2 / 95 \\
7 / 6 / 95 \\
9 / 13 / 95\end{array}$ & $\begin{array}{l}16.8 \\
15.7 \\
14.4 \\
12.1 \\
10.4 \\
10.37 \\
10.64 \\
12.37\end{array}$ & $\begin{array}{l}1,440.2 \\
1,441.3 \\
1,442.6 \\
1,444.9 \\
1,446.6 \\
1,446.63 \\
1,446.36 \\
1,444.63\end{array}$ & $\begin{array}{l}\text { EMWD } \\
\text { EMWD } \\
\text { EMWD } \\
\text { EMWD } \\
\text { EMWD } \\
\text { USGS } \\
\text { USGS } \\
\text { USGS }\end{array}$ \\
\hline $5 S / 2 W-30 C 1$ & & 370 & $355.8(5 / 16 / 95)$ & $\begin{array}{l}130-190 \\
210-230 \\
270-370\end{array}$ & 1,452 & $\begin{array}{l}5 / 16 / 95 \\
9 / 13 / 95\end{array}$ & $\begin{array}{r}8.50 \\
15.12\end{array}$ & $\begin{array}{l}1,443.50 \\
1,436.88\end{array}$ & $\begin{array}{l}\text { USGS } \\
\text { USGS }\end{array}$ \\
\hline $5 S 2 W-30 D 2$ & & - & - & -- & 1,457 & $\begin{array}{r}1 / 23 / 51 \\
1 / 24 / 52 \\
2 / 3 / 53 \\
12 / 21 / 53\end{array}$ & $\begin{array}{l}38.7 \\
41.7 \\
42.4 \\
43.6\end{array}$ & $\begin{array}{l}1,418.3 \\
1,415.3 \\
1,414.6 \\
1,413.4\end{array}$ & $\begin{array}{l}\text { DWR } \\
\text { DWR } \\
\text { DWR } \\
\text { DWR }\end{array}$ \\
\hline $5 S / 2 W-30 G 2$ & Winchester \#1 & 70 & $70.7(5 / 23 / 95)$ & $50-70$ & 1,447 & $\begin{array}{r}12 / 93 \\
3 / 94 \\
9 / 94 \\
3 / 95 \\
5 / 23 / 95 \\
6 / 95 \\
7 / 7 / 95 \\
9 / 13 / 95\end{array}$ & $\begin{array}{l}10.6 \\
9.0 \\
8.8 \\
4.2 \\
5.65 \\
6.2 \\
6.95 \\
9.84\end{array}$ & $\begin{array}{l}1,436.4 \\
1,438.0 \\
1,438.2 \\
1,442.8 \\
1,441.35 \\
1,440.8 \\
1,440.05 \\
1,437.16\end{array}$ & $\begin{array}{l}\text { EMWD } \\
\text { EMWD } \\
\text { EMWD } \\
\text { EMWD } \\
\text { USGS } \\
\text { EMWD } \\
\text { USGS } \\
\text { USGS }\end{array}$ \\
\hline $5 S / 2 W-30 G 3$ & Winchester \#2 & 72 & $71.4(5 / 23 / 95)$ & $52-72$ & 1,449 & $\begin{array}{r}12 / 93 \\
3 / 94 \\
9 / 94 \\
3 / 95 \\
5 / 23 / 95 \\
6 / 95 \\
7 / 6 / 95 \\
9 / 13 / 95\end{array}$ & $\begin{array}{c}16.7 \\
11.3 \\
11.5 \\
6.7 \\
6.96 \\
7.5 \\
8.00 \\
13.33\end{array}$ & $\begin{array}{l}1,432.3 \\
1,437.7 \\
1,437.5 \\
1,442.3 \\
1,442.04 \\
1,441.5 \\
1,441.00 \\
1,435.67\end{array}$ & $\begin{array}{l}\text { EMWD } \\
\text { EMWD } \\
\text { EMWD } \\
\text { EMWD } \\
\text { USGS } \\
\text { EMWD } \\
\text { USGS } \\
\text { USGS }\end{array}$ \\
\hline
\end{tabular}


Table 3. Water-level data for selected wells in the Winchester, Menifee, and south Perris ground-water subbasins, CaliforniaContinued

\begin{tabular}{|c|c|c|c|c|c|c|c|c|c|}
\hline \multirow[t]{2}{*}{ Well No. } & \multirow{2}{*}{$\begin{array}{l}\text { Other known } \\
\text { designation }\end{array}$} & \multicolumn{2}{|c|}{ Well depth (ft) } & \multirow{2}{*}{$\begin{array}{l}\text { Perforations } \\
\text { (ft blw LSD) }\end{array}$} & \multirow[t]{2}{*}{ Altitude } & \multirow[t]{2}{*}{ Date } & \multicolumn{2}{|c|}{ Water Level } & \multirow{2}{*}{$\begin{array}{c}\text { Source of } \\
\text { data }\end{array}$} \\
\hline & & Original & Sounded (date) & & & & $\overline{\text { (ft blw LSD) }}$ & Altitude & \\
\hline \multirow[t]{8}{*}{$5 \mathrm{~S} / 2 \mathrm{~W}-30 \mathrm{H} 1$} & Winchester \#6 & 70 & $69.1(5 / 11 / 95)$ & $50-70$ & $1,462.5$ & $12 / 93$ & 22.4 & $1,440.1$ & EMWD \\
\hline & & & & & & $3 / 94$ & 18.0 & $1,444.5$ & EMWD \\
\hline & & & & & & 9/94 & 18.6 & $1,443.9$ & EMWD \\
\hline & & & & & & 3/95 & 10.6 & $1,451.9$ & EMWD \\
\hline & & & & & & $5 / 11 / 95$ & 9.56 & $1,452.94$ & USGS \\
\hline & & & & & & $6 / 95$ & 10.9 & $1,451.6$ & EMWD \\
\hline & & & & & & $7 / 6 / 95$ & 12.56 & $1,449.94$ & USGS \\
\hline & & & & & & $9 / 11 / 95$ & 19.53 & $1,442.97$ & USGS \\
\hline \multirow[t]{7}{*}{$5 \mathrm{~S} / 2 \mathrm{~W}-30 \mathrm{H} 2$} & Winchester \#7 & 70 & $69.9(5 / 11 / 95)$ & $50-70$ & 1,459 & $12 / 93$ & 19.5 & $1,439.5$ & EMWD \\
\hline & & & & & & $3 / 94$ & 16.2 & $1,442.8$ & EMWD \\
\hline & & & & & & 9/94 & 13.8 & $1,445.2$ & EMWD \\
\hline & & & & & & 3/95 & 10.1 & $1,448.9$ & EMWD \\
\hline & & & & & & $5 / 11 / 95$ & 9.20 & $1,449.80$ & USGS \\
\hline & & & & & & $6 / 95$ & 9.8 & $1,449.2$ & EMWD \\
\hline & & & & & & $9 / 11 / 95$ & 16.59 & $1,442.41$ & USGS \\
\hline \multirow[t]{8}{*}{$5 \mathrm{~S} / 2 \mathrm{~W}-30 \mathrm{H} 3$} & Winchester \#8 & 70 & $70.9(5 / 23 / 95)$ & $50-70$ & 1,453 & $12 / 93$ & 15.1 & $1,437.9$ & EMWD \\
\hline & & & & & & $3 / 94$ & 8.0 & $1,445.0$ & EMWD \\
\hline & & & & & & 9/94 & 9.6 & 1.443 .4 & EMWD \\
\hline & & & & & & 3/95 & 3.8 & $1,449.2$ & EMWD \\
\hline & & & & & & $5 / 23 / 95$ & 5.10 & $1,447.90$ & USGS \\
\hline & & & & & & $6 / 95$ & 5.2 & $1,447.8$ & EMWD \\
\hline & & & & & & $7 / 6 / 95$ & 5.65 & $1,447.35$ & USGS \\
\hline & & & & & & $9 / 11 / 95$ & 12.93 & $1,440.07$ & USGS \\
\hline \multirow[t]{8}{*}{$5 S / 2 W-31 H 1$} & $5 S / 2 W-31 R 1^{\star *}$ & - & - & - & $1,457.1^{*}$ & $1 / 13 / 41$ & $26.0^{*}$ & $1,431.1$ & DWR \\
\hline & & & & & & $1 / 15 / 43$ & $21.2^{\star}$ & $1,435.9$ & DWR \\
\hline & & & & & & $1 / 17 / 44$ & $18.7^{\star}$ & $1,438.4$ & DWR \\
\hline & & & & & & $1 / 9 / 45$ & $19.5^{\star}$ & $1,437.6$ & DWR \\
\hline & & & & & & $1 / 10 / 46$ & $22.9^{*}$ & $1,434.2$ & DWR \\
\hline & & & & & & $1 / 9 / 47$ & $26.0^{\star}$ & $1,431.1$ & DWR \\
\hline & & & & & & $1 / 22 / 48$ & $26.5^{\star}$ & $1,430.6$ & DWR \\
\hline & & & & & & $2 / 6 / 53$ & $22.0^{\star}$ & $1,435.1$ & DWR \\
\hline \multirow[t]{5}{*}{$5 S / 2 W-31 H 2$} & $5 S / 2 W-31 R 2^{\star \star}$ & - & $104 R$ (1953) & - & 1,455 & $1 / 18 / 50$ & 28.1 & $1,426.9$ & DWR \\
\hline & & & & & & $1 / 24 / 51$ & 30.1 & $1,424.9$ & DWR \\
\hline & & & & & & 1/17/52 & 30.1 & $1,424.9$ & DWR \\
\hline & & & & & & $2 / 6 / 53$ & 28.9 & $1,426.1$ & DWR \\
\hline & & & & & & $12 / 20 / 53$ & 32.2 & $1,422.8$ & DWR \\
\hline \multirow[t]{11}{*}{$5 S / 2 W-32 A$} & $5 S / 2 W-32 R 3^{* *}$ & - & - & -- & $1,454.7$ & $1 / 8 / 42$ & 15.1 & $1,439.6$ & DWR \\
\hline & & & & & & $1 / 15 / 43$ & 19.6 & $1,435.1$ & DWR \\
\hline & & & & & & $1 / 9 / 45$ & 13.0 & $1,441.7$ & DWR \\
\hline & & & & & & $1 / 10 / 46$ & 15.2 & $1,439.5$ & DWR \\
\hline & & & & & & $1 / 9 / 47$ & 13.0 & $1,441.7$ & DWR \\
\hline & & & & & & $1 / 22 / 48$ & 17.5 & $1,437.2$ & DWR \\
\hline & & & & & & $12 / 16 / 48$ & 18.5 & $1,436.2$ & DWR \\
\hline & & & & & & $1 / 18 / 50$ & 23.3 & $1,431.4$ & DWR \\
\hline & & & & & & $1 / 24 / 51$ & 26.0 & $1,428.7$ & DWR \\
\hline & & & & & & $1 / 17 / 52$ & 27.5 & $1,427.2$ & DWR \\
\hline & & & & & & $2 / 11 / 53$ & 24.4 & $1,430.3$ & DWR \\
\hline $5 S / 2 W-32 G$ & $5 S / 2 W-32 R 1^{\star \star}$ & 90 & - & - & $1,457.6$ & $1 / 8 / 42$ & 14.9 & $1,442.7$ & DWR \\
\hline & & & & & & $1 / 15 / 43$ & 18.3 & $1,439.3$ & DWR \\
\hline & & & & & & $1 / 17 / 44$ & 15.0 & $1,442.6$ & DWR \\
\hline & & & & & & $1 / 9 / 45$ & 15.3 & $1,442.3$ & DWR \\
\hline & & & & & & $1 / 10 / 46$ & 16.8 & $1,440.8$ & DWR \\
\hline & & & & & & $1 / 9 / 47$ & 18.1 & $1,439.5$ & DWR \\
\hline & & & & & & $1 / 22 / 48$ & 20.6 & $1,437.0$ & DWR \\
\hline & & & & & & $12 / 11 / 48$ & 24.6 & $1,433.0$ & DWR \\
\hline & & & & & & $1 / 18 / 50$ & 24.6 & $1,433.0$ & DWR \\
\hline & & & & & & $1 / 24 / 51$ & 26.6 & $1,431.0$ & DWR \\
\hline & & & & & & $1 / 17 / 52$ & 28.4 & $1,429.2$ & DWR, de \\
\hline
\end{tabular}


Table 3. Water-level data for selected wells in the Winchester, Menifee, and south Perris ground-water subbasins, CaliforniaContinued

\begin{tabular}{|c|c|c|c|c|c|c|c|c|c|}
\hline \multirow[t]{2}{*}{ Well No. } & \multirow{2}{*}{$\begin{array}{l}\text { Other known } \\
\text { designation }\end{array}$} & \multicolumn{2}{|c|}{ Well depth (ft) } & \multirow{2}{*}{$\begin{array}{l}\text { Perforations } \\
\text { (ft blw LSD) }\end{array}$} & \multirow[t]{2}{*}{ Altitude } & \multirow[t]{2}{*}{ Date } & \multicolumn{2}{|c|}{ Water Level } & \multirow{2}{*}{$\begin{array}{c}\text { Source of } \\
\text { data }\end{array}$} \\
\hline & & Original & Sounded (date) & & & & (ft blw LSD) & Altitude & \\
\hline \multirow[t]{7}{*}{$5 S / 2 W-33 C 1$} & & 438 & $71.2(5 / 16 / 95)$ & $44-54$ & 1,462 & $12 / 30 / 92$ & 16.44 & $1,445.56$ & USGS \\
\hline & & & & $62-73$ & & $5 / 13 / 93$ & 8.59 & $1,453.41$ & USGS \\
\hline & & & & $75-85$ & & $2 / 10 / 94$ & 9.49 & $1,452.51$ & USGS \\
\hline & & & & $96-110$ & & $6 / 16 / 94$ & 10.31 & $1,451.69$ & USGS \\
\hline & & & & $124-133$ & & $11 / 17 / 94$ & 11.52 & $1,450.48$ & USGS \\
\hline & & & & $216-240$ & & $5 / 16 / 95$ & 8.22 & $1,453.78$ & USGS \\
\hline & & & & $287-403$ & & $9 / 13 / 95$ & 10.45 & $1,451.55$ & USGS \\
\hline \multirow[t]{6}{*}{ 5S/2W-33D1 } & & 270 & $145.1(5 / 16 / 95)$ & -- & 1,458 & $5 / 13 / 93$ & 7.32 & $1,450.68$ & USGS \\
\hline & & & & & & $12 / 30 / 93$ & 15.30 & $1,442.70$ & USGS \\
\hline & & & & & & $2 / 10 / 94$ & 8.40 & $1,449.60$ & USGS \\
\hline & & & & & & $6 / 16 / 94$ & 9.31 & $1,448.69$ & USGS \\
\hline & & & & & & $11 / 17 / 94$ & 10.71 & $1,447.29$ & USGS \\
\hline & & & & & & $5 / 16 / 95$ & 6.13 & $1,451.87$ & USGS \\
\hline 5S/2W-33D1 & & & & & & $9 / 13 / 95$ & 8.44 & $1,449.56$ & USGS \\
\hline \multirow[t]{9}{*}{$5 S / 3 W-2 M 1$} & & 300 & $229.0(4 / 28 / 95)$ & -- & 1,445 & $1 / 8 / 42$ & 129.0 & $1,316.0$ & DWR \\
\hline & & & & & & $1 / 13 / 43$ & 131.3 & $1,313.7$ & DWR \\
\hline & & & & & & $1 / 19 / 44$ & 134.9 & $1,310.1$ & DWR \\
\hline & & & & & & $1 / 17 / 46$ & 141.5 & $1,303.5$ & DWR \\
\hline & & & & & & $1 / 8 / 47$ & 144.1 & $1,300.9$ & DWR \\
\hline & & & & & & $1 / 22 / 48$ & 147.0 & $1,298.0$ & DWR \\
\hline & & & & & & $1 / 24 / 52$ & 164.7 & $1,280.3$ & DWR \\
\hline & & & & & & $4 / 28 / 95$ & 140.31 & $1,304.69$ & USGS \\
\hline & & & & & & $9 / 13 / 95$ & 136.64 & $1,308.36$ & USGS \\
\hline \multirow[t]{10}{*}{$5 S / 3 W-2 R 1$} & & 99 & - & - & 1,533 & $1 / 8 / 42$ & 22.1 & $1,510.9$ & DWR \\
\hline & & & & & & $1 / 13 / 43$ & 22.5 & $1,510.5$ & DWR \\
\hline & & & & & & $1 / 19 / 44$ & 20.0 & $1,513.0$ & DWR \\
\hline & & & & & & $1 / 11 / 45$ & 19.7 & $1,513.3$ & DWR \\
\hline & & & & & & $1 / 17 / 46$ & 21.3 & $1,511.7$ & DWR \\
\hline & & & & & & $1 / 8 / 47$ & 23.3 & $1,509.7$ & DWR \\
\hline & & & & & & $1 / 22 / 48$ & 25.1 & $1,507.9$ & DWR \\
\hline & & & & & & $1 / 26 / 51$ & 29.5 & $1,503.5$ & DWR \\
\hline & & & & & & $1 / 24 / 52$ & 34.4 & $1,498.6$ & DWR \\
\hline & & & & & & $2 / 9 / 53$ & 44.9 & $1,488.1$ & DWR \\
\hline \multirow[t]{6}{*}{$5 S / 3 W-3 C 1$} & B-6 & 260 & $250.9(3 / 31 / 95)$ & $230-250$ & 1,420 & $3 / 94$ & 120.9 & $1,299.1$ & EMWD \\
\hline & & & & & & $9 / 94$ & 117.8 & $1,302.2$ & EMWD \\
\hline & & & & & & $3 / 95$ & 113.9 & $1,306.1$ & EMWD \\
\hline & & & & & & $3 / 31 / 95$ & 113.61 & $1,306.39$ & USGS \\
\hline & & & & & & $6 / 95$ & 111.1 & $1,308.9$ & EMWD \\
\hline & & & & & & $9 / 11 / 95$ & 109.26 & $1,310.74$ & USGS \\
\hline \multirow[t]{6}{*}{$5 S / 3 W-3 L 1$} & B-7 & 260 & $252.0(3 / 31 / 95)$ & $230-250$ & 1,425 & $3 / 94$ & 116.3 & $1,308.7$ & EMWD \\
\hline & & & & & & 9/94 & 113.7 & $1,311.3$ & EMWD \\
\hline & & & & & & $3 / 95$ & 110.0 & $1,315.0$ & EMWD \\
\hline & & & & & & $3 / 31 / 95$ & 109.73 & $1,315.27$ & USGS \\
\hline & & & & & & $6 / 95$ & 107.6 & $1,317.4$ & EMWD \\
\hline & & & & & & $9 / 11 / 95$ & 105.98 & $1,319.02$ & USGS \\
\hline $5 S / 3 W-3 N 1$ & $A-1$ & 865 & $577.8(5 / 3 / 95)$ & $290-310$ & 1,415 & $3 / 94$ & 100.2 & $1,314.8$ & EMWD \\
\hline & & & & $555-575$ & & 9/94 & 99.2 & $1,315.8$ & EMWD \\
\hline & & & & & & $3 / 95$ & 94.9 & $1,320.1$ & EMWD \\
\hline & & & & & & $3 / 31 / 95$ & 94.64 & $1,320.36$ & USGS \\
\hline & & & & & & $5 / 3 / 95$ & 94.02 & $1,320.98$ & USGS \\
\hline & & & & & & $9 / 13 / 95$ & 91.92 & $1,323.08$ & USGS \\
\hline 5S/3W-3R1 & & 540 & -- & $180-540$ & 1,435 & $1 / 11 / 93$ & 142.50 & $1,292.5$ & EMWD \\
\hline $5 S / 3 W-4 A 1$ & B-5 & 260 & $209.4(4 / 14 / 95)$ & $190-210$ & 1,415 & $3 / 94$ & 108.2 & $1,306.8$ & EMWD \\
\hline & & & & & & $9 / 94$ & 105.1 & $1,309.9$ & EMWD \\
\hline & & & & & & 3/95 & 101.6 & $1,313.4$ & EMWD \\
\hline & & & & & & 4/14/95 & 101.10 & $1,313.90$ & USGS \\
\hline & & & & & & $6 / 95$ & 99.3 & $1,315.7$ & EMWD \\
\hline & & & & & & $9 / 11 / 95$ & 97.75 & $1,317.25$ & USGS \\
\hline
\end{tabular}


Table 3. Water-level data for selected wells in the Winchester, Menifee, and south Perris ground-water subbasins, CaliforniaContinued

\begin{tabular}{|c|c|c|c|c|c|c|c|c|c|}
\hline \multirow[t]{2}{*}{ Well No. } & \multirow{2}{*}{$\begin{array}{l}\text { Other known } \\
\text { designation }\end{array}$} & \multicolumn{2}{|c|}{ Well depth (ft) } & \multirow{2}{*}{$\begin{array}{l}\text { Perforations } \\
\text { (ft blw LSD) }\end{array}$} & \multirow[t]{2}{*}{ Altitude } & \multirow[t]{2}{*}{ Date } & \multicolumn{2}{|c|}{ Water Level } & \multirow{2}{*}{ - $\begin{array}{c}\text { Source of } \\
\text { data }\end{array}$} \\
\hline & & Original & Sounded (date) & & & & (ft b/w LSD) & Altitude & \\
\hline \multirow[t]{6}{*}{$5 S / 3 W-4 M 1$} & B-1 & 260 & $251.3(4 / 13 / 95)$ & $230-250$ & 1,412 & $3 / 94$ & 93.8 & $1,318.2$ & EMWD \\
\hline & & & & & & $9 / 94$ & 93.1 & $1,318.9$ & EMWD \\
\hline & & & & & & $3 / 95$ & 88.3 & $1,323.7$ & EMWD \\
\hline & & & & & & 4/13/95 & 87.70 & $1,324.30$ & USGS \\
\hline & & & & & & $6 / 26 / 95$ & 88.55 & $1,323.45$ & USGS \\
\hline & & & & & & $9 / 13 / 95$ & 85.30 & $1,326.70$ & USGS \\
\hline \multirow[t]{2}{*}{$5 S / 3 W-5 A 1$} & $5 S / 3 W-5 R 3^{* *}$ & -- & - & -- & 1,413 & $1 / 11 / 50$ & 131.5 & $1,281.5$ & DWR \\
\hline & & & & & & $1 / 26 / 51$ & 139.9 & $1,273.1$ & RCFCD \\
\hline \multirow[t]{2}{*}{$5 S / 3 W-5 A 1$} & & & & & & $1 / 24 / 52$ & 150.5 & $1,262.5$ & RCFCD \\
\hline & & & & & & $2 / 9 / 53$ & 152.7 & $1,260.3$ & DWR, sealed \\
\hline \multirow[t]{12}{*}{$5 S / 3 W-5 B 2$} & & - & -. & -. & 1,413 & $3 / 13 / 42$ & 98.0 & $1,315.0$ & DWR \\
\hline & & & & & & $1 / 13 / 43$ & 104.4 & $1,308.6$ & DWR \\
\hline & & & & & & $1 / 19 / 44$ & 110.6 & $1,302.4$ & DWR \\
\hline & & & & & & $1 / 16 / 45$ & 106.6 & $1,306.4$ & DWR \\
\hline & & & & & & $1 / 8 / 46$ & 105.7 & $1,307.3$ & DWR \\
\hline & & & & & & $1 / 7 / 47$ & 111.8 & $1,301.2$ & DWR \\
\hline & & & & & & $1 / 15 / 48$ & 116.6 & $1,296.4$ & DWR \\
\hline & & & & & & $12 / 16 / 48$ & 126.0 & $1,287.0$ & DWR \\
\hline & & & & & & $1 / 11 / 50$ & 131.0 & $1,282.0$ & DWR \\
\hline & & & & & & $1 / 26 / 51$ & 140.1 & $1,272.9$ & DWR \\
\hline & & & & & & $1 / 24 / 52$ & 157.5 & $1,255.5$ & DWR \\
\hline & & & & & & $2 / 9 / 53$ & 152.7 & $1,260.3$ & DWR, sealed \\
\hline \multirow[t]{15}{*}{$5 S / 3 W-5 M 1$} & $5 S / 3 W-5 R 1^{* *}$ & -- & - & - & $1,414.8$ & $1 / 7 / 42$ & 21.7 & $1,393.1$ & DWR \\
\hline & & & & & & $1 / 13 / 43$ & 27.8 & $1,387.0$ & DWR \\
\hline & & & & & & $1 / 18 / 44$ & 25.6 & $1,389.2$ & DWR \\
\hline & & & & & & $1 / 11 / 45$ & 21.7 & $1,393.1$ & DWR \\
\hline & & & & & & $1 / 8 / 46$ & 21.8 & $1,393.0$ & DWR \\
\hline & & & & & & $1 / 8 / 47$ & 23.9 & $1,390.9$ & DWR \\
\hline & & & & & & $1 / 15 / 48$ & 23.6 & $1,391.2$ & DWR \\
\hline & & & & & & $12 / 16 / 48$ & 25.6 & $1,389.2$ & DWR \\
\hline & & & & & & $1 / 11 / 50$ & 26.7 & $1,388.1$ & DWR \\
\hline & & & & & & $1 / 26 / 51$ & 27.0 & $1,387.8$ & DWR \\
\hline & & & & & & $1 / 30 / 52$ & 28.0 & $1,386.8$ & DWR \\
\hline & & & & & & $2 / 11 / 53$ & 27.9 & $1,386.9$ & DWR \\
\hline & & & & & & $12 / 21 / 53$ & 28.8 & $1,386.0$ & DWR \\
\hline & & & & & & $11 / 9 / 54$ & 24.6 & $1,390.2$ & DWR \\
\hline & & & & & & $4 / 10 / 55$ & 27.8 & $1,387.0$ & RCFCD, collapsed \\
\hline \multirow[t]{7}{*}{$5 S / 3 W-6 G 1$} & $5 S / 3 W-6 R 1^{* *}$ & 56 & - & -- & 1,443 & $1 / 8 / 42$ & 44.8 & $1,398.2$ & DWR \\
\hline & & & & & & $1 / 13 / 43$ & 45.8 & $1,397.2$ & DWR \\
\hline & & & & & & $1 / 15 / 48$ & 44.6 & $1,398.4$ & RCFCD \\
\hline & & & & & & $1 / 26 / 51$ & 48.9 & $1,394.1$ & RCFCD \\
\hline & & & & & & $1 / 30 / 52$ & 49.6 & $1,393.4$ & DWR \\
\hline & & & & & & $1 / 23 / 53$ & 49.7 & $1,393.3$ & DWR \\
\hline & & & & & & $12 / 21 / 53$ & 49.9 & $1,393.1$ & DWR,collapsed \\
\hline $5 S / 3 W-6 P 1$ & $5 S / 3 W-6 R 2^{\star \star}$ & -- & - & - & 1,435 & $1 / 7 / 42$ & 25.5 & $1,409.5$ & DWR \\
\hline & & & & & & $1 / 13 / 43$ & 26.9 & $1,408.1$ & DWR \\
\hline & & & & & & $1 / 18 / 44$ & 27.5 & $1,407.5$ & DWR \\
\hline & & & & & & $1 / 11 / 45$ & 24.4 & $1,410.6$ & DWR \\
\hline & & & & & & $1 / 8 / 46$ & 24.3 & $1,410.7$ & DWR \\
\hline & & & & & & $1 / 8 / 47$ & 26.2 & $1,408.8$ & DWR \\
\hline & & & & & & $1 / 15 / 48$ & 28.9 & $1,406.1$ & DWR \\
\hline & & & & & & $12 / 16 / 48$ & 31.2 & $1,403.8$ & DWR \\
\hline & & & & & & $1 / 11 / 50$ & 30.9 & $1,404.1$ & DWR \\
\hline & & & & & & $1 / 26 / 51$ & 35.4 & $1,399.6$ & DWR \\
\hline & & & & & & $1 / 30 / 52$ & 37.8 & $1,397.2$ & DWR \\
\hline & & & & & & $1 / 23 / 53$ & 36.1 & $1,398.9$ & DWR \\
\hline & & & & & & $12 / 21 / 53$ & 37.8 & $1,397.2$ & DWR, destroyed \\
\hline $5 S / 3 W-7 J 1$ & $5 S / 3 W-7 R 2^{\star \star}$ & - & - & - & 1,407 & $1 / 7 / 42$ & 12.0 & $1,395.0$ & RCFCD \\
\hline & & & & & & $1 / 13 / 43$ & 14.8 & $1,392.2$ & RCFCD \\
\hline
\end{tabular}


Table 3. Water-level data for selected wells in the Winchester, Menifee, and south Perris ground-water subbasins, California-Continued

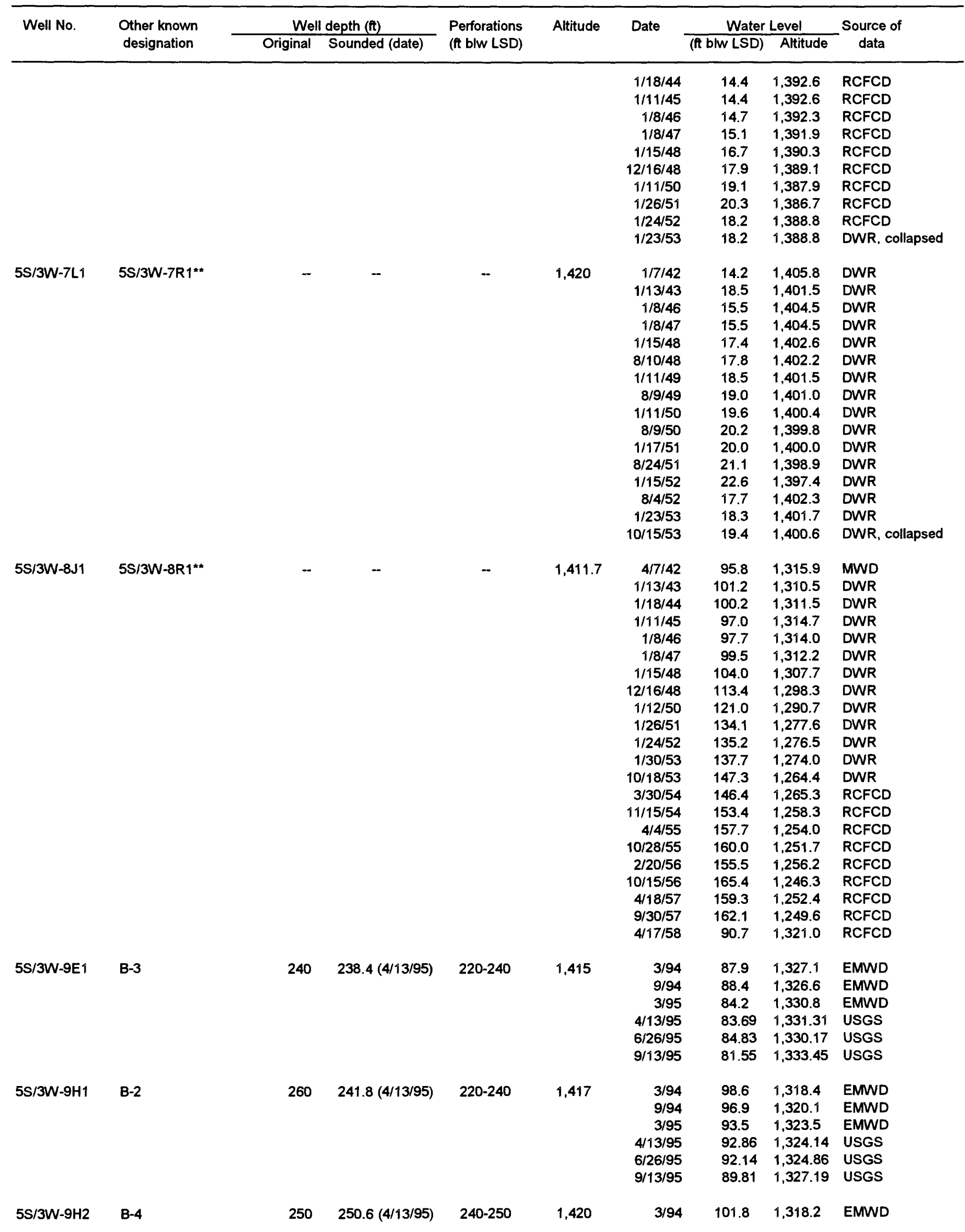


Tab/e 3. Water-level data for selected wells in the Winchester, Menifee, and south Perris ground-water subbasins, CaliforniaContinued

\begin{tabular}{|c|c|c|c|c|c|c|c|c|c|}
\hline \multirow[t]{2}{*}{ Well No. } & \multirow{2}{*}{$\begin{array}{l}\text { Other known } \\
\text { designation }\end{array}$} & \multicolumn{2}{|c|}{ Well depth (ft) } & \multirow{2}{*}{$\begin{array}{l}\text { Perforations } \\
\text { (ft blw LSD) }\end{array}$} & \multirow[t]{2}{*}{ Altitude } & \multirow[t]{2}{*}{ Date } & \multicolumn{2}{|c|}{ Water Level } & \multirow{2}{*}{$\begin{array}{c}\text { Source of } \\
\text { data }\end{array}$} \\
\hline & & Original & Sounded (date) & & & & (ft blw LSD) & Altitude & \\
\hline & & & & & & 9/94 & 99.2 & $1,320.8$ & EMWD \\
\hline & & & & & & $3 / 95$ & 93.2 & $1,326.8$ & EMWD \\
\hline & & & & & & $4 / 13 / 95$ & 96.00 & $1,324.00$ & USGS \\
\hline & & & & & & $6 / 26 / 95$ & 96.28 & $1,323.72$ & USGS \\
\hline & & & & & & $9 / 13 / 95$ & 93.60 & $1,326.40$ & USGS \\
\hline $5 S / 3 W-9 Q 1$ & & 600 & - & $180-600$ & 1,421 & $4 / 19 / 95$ & 88.30 & $1,332.70$ & USGS \\
\hline \multirow[t]{7}{*}{ 5S/3W-9R1 } & $5 S / 3 W-9 R 2 * *$ & - & -- & -- & $1,421.3$ & $1 / 7 / 42$ & 105.3 & $1,316.0$ & DWR \\
\hline & & & & & & $1 / 13 / 43$ & 109.1 & $1,312.2$ & DWR \\
\hline & & & & & & $1 / 18 / 44$ & 109.0 & $1,312.3$ & DWR \\
\hline & & & & & & $1 / 11 / 45$ & 109.2 & $1,312.1$ & DWR \\
\hline & & & & & & $1 / 17 / 46$ & 109.0 & $1,312.3$ & DWR \\
\hline & & & & & & $1 / 14 / 50$ & 133.0 & $1,288.3$ & DWR \\
\hline & & & & & & $1 / 29 / 52$ & 143.2 & $1,278.1$ & DWR, destroyed \\
\hline \multirow[t]{6}{*}{ 5S/3W-10M1 } & & -- & -- & - & $1,423.5$ & $1 / 13 / 42$ & 109.9 & $1,313.6$ & RCFCD \\
\hline & & & & & & $1 / 13 / 43$ & 115.3 & $1,308.2$ & RCFCD \\
\hline & & & & & & $1 / 17 / 46$ & 119.4 & $1,304.1$ & RCFCD \\
\hline & & & & & & $1 / 14 / 50$ & 134.7 & $1,288.8$ & RCFCD \\
\hline & & & & & & $1 / 26 / 51$ & 146.3 & $1,277.2$ & DWR \\
\hline & & & & & & $1 / 31 / 52$ & 149.3 & $1,274.2$ & DWR, destroyed \\
\hline \multirow[t]{3}{*}{ 5S/3W-10M2 } & & - & $333.7(5 / 5 / 95)$ & - & 1,422 & $1 / 12 / 93$ & 113.1 & $1,308.9$ & EMWD \\
\hline & & & & & & $2 / 28 / 95$ & 97.8 & $1,324.2$ & EMWD \\
\hline & & & & & & $5 / 5 / 95$ & 96.22 & $1,325.78$ & USGS \\
\hline \multirow[t]{2}{*}{$5 S / 3 W-10 N 1$} & & - & - & -- & 1,425 & $6 / 2 / 95$ & 97.98 & $1,327.02$ & USGS \\
\hline & & & & & & $9 / 13 / 95$ & 95.63 & $1,329.37$ & USGS \\
\hline 5S/3W-11D1 & & 227 & - & - & 1,465 & $5 / 5 / 95$ & 134.26 & $1,330.74$ & USGS \\
\hline \multirow[t]{2}{*}{$5 S / 3 W-11 E 2$} & $5 S / 3 W-11 R 4^{\star *}$ & 400 & - & -- & 1,464 & $1 / 31 / 52$ & 206.7 & $1,257.3$ & DWR \\
\hline & & & & & & $2 / 11 / 53$ & 219.2 & $1,244.8$ & DWR \\
\hline $5 S / 3 W-11 E 3$ & $5 S / 3 W-11 R 5^{\star \star}$ & 600 & - & - & 1.463 & 1/31/52 & 206.6 & $1,256.4$ & DWR \\
\hline \multirow[t]{9}{*}{$5 S / 3 W-11 K 1$} & $5 S / 3 W-11 R 2^{\star \star}$ & - & - & - & 1,482 & $1 / 13 / 42$ & 171.2 & $1,310.8$ & DWR \\
\hline & & & & & & $1 / 13 / 43$ & 187.6 & $1,294.4$ & DWR \\
\hline & & & & & & $1 / 19 / 44$ & 198.4 & $1,283.6$ & DWR \\
\hline & & & & & & $1 / 11 / 45$ & 201.8 & $1,280.2$ & DWR \\
\hline & & & & & & $1 / 17 / 46$ & 197.3 & $1,284.7$ & DWR \\
\hline & & & & & & $1 / 8 / 47$ & 198.4 & $1,283.6$ & DWR \\
\hline & & & & & & $1 / 21 / 48$ & 198.1 & $1,283.9$ & DWR \\
\hline & & & & & & $1 / 24 / 52$ & 213.3 & $1,268.7$ & DWR \\
\hline & & & & & & $2 / 11 / 53$ & 214.6 & $1,267.4$ & DWR \\
\hline \multirow[t]{8}{*}{$5 S / 3 W-11 R 1$} & & 135 & - & - & 1,476 & $1 / 7 / 42$ & 68.0 & $1,408.0$ & DWR \\
\hline & & & & & & $1 / 13 / 43$ & 70.2 & $1,405.8$ & DWR \\
\hline & & & & & & $1 / 18 / 44$ & 71.5 & $1,404.5$ & DWR \\
\hline & & & & & & $1 / 11 / 45$ & 73.2 & $1,402.8$ & DWR \\
\hline & & & & & & $1 / 16 / 46$ & 73.3 & $1,402.7$ & DWR \\
\hline & & & & & & $1 / 8 / 47$ & 71.6 & $1,404.4$ & DWR \\
\hline & & & & & & $1 / 20 / 48$ & 73.3 & $1,402.7$ & DWR \\
\hline & & & & & & $3 / 16 / 95$ & 52.9 & $1,423.1$ & EMWD \\
\hline \multirow[t]{4}{*}{$5 S / 3 W-13 C 1$} & & -- & - & - & 1,489 & $5 / 16 / 88$ & 56.0 & 1.433 .0 & EMWD \\
\hline & & & & & & $1 / 12 / 93$ & 62.8 & $1,426.2$ & EMWD \\
\hline & & & & & & $4 / 2 / 93$ & 62.4 & $1,426.6$ & EMWD \\
\hline & & & & & & $2 / 28 / 95$ & 61.6 & $1,427.4$ & EMWD \\
\hline \multirow[t]{3}{*}{$5 S / 3 W-13 N 1$} & & 433 & $142.0(5 / 22 / 95)$ & $250-433$ & 1,475 & $5 / 22 / 95$ & 52.02 & $1,422.98$ & USGS \\
\hline & & & & & & $6 / 23 / 95$ & 52.01 & $1,422.99$ & USGS \\
\hline & & & & & & $9 / 28 / 95$ & 52.10 & $1,422.90$ & USGS \\
\hline $5 S / 3 W-14 L 1$ & & 200 & - & $80-200$ & 1,444 & $6 / 5 / 95$ & 71.83 & $1,372.17$ & USGS \\
\hline
\end{tabular}


Table 3. Water-level data for selected wells in the Winchester, Menifee, and south Perris ground-water subbasins, CaliforniaContinued

\begin{tabular}{|c|c|c|c|c|c|c|c|c|c|}
\hline \multirow[t]{2}{*}{ Well No. } & \multirow{2}{*}{$\begin{array}{l}\text { Other known } \\
\text { designation }\end{array}$} & \multicolumn{2}{|c|}{ Well depth (ft) } & \multirow{2}{*}{$\begin{array}{l}\text { Perforations } \\
\text { (ft blw LSD) }\end{array}$} & \multirow[t]{2}{*}{ Altitude } & \multirow[t]{2}{*}{ Date } & \multicolumn{2}{|c|}{ Water Level } & \multirow{2}{*}{$\begin{array}{c}\text { Source of } \\
\text { data }\end{array}$} \\
\hline & & Original & Sounded (date) & & & & (ft blw LSD) & Altitude & \\
\hline $5 S / 3 W-15 H 1$ & & 150 & - & $110-150$ & 1,429 & $\begin{array}{r}11 / 19 / 93 \\
5 / 5 / 95\end{array}$ & $\begin{array}{l}110.20 \\
101.32\end{array}$ & $\begin{array}{l}1,318.80 \\
1,327.68\end{array}$ & $\begin{array}{l}\text { USGS } \\
\text { USGS }\end{array}$ \\
\hline 5S/3W-15P1 & & 185 & - & $118-185$ & 1,428 & 6/6/95 & 86.38 & $1,341.62$ & USGS \\
\hline $5 S / 3 W-15 Q 1$ & & 380 & -- & $\begin{array}{l}180-220 \\
300-380\end{array}$ & 1,439 & $5 / 22 / 95$ & 33.49 & $1,405.51$ & USGS \\
\hline $5 S / 3 W-24 C 1$ & & 505 & -- & 265-505 & 1,480 & $\begin{array}{r}1 / 4 / 94 \\
3 / 13 / 95\end{array}$ & $\begin{array}{l}72.56 \\
69.50\end{array}$ & $\begin{array}{l}1,407.44 \\
1,410.50\end{array}$ & $\begin{array}{l}\text { USGS } \\
\text { EMWD }\end{array}$ \\
\hline $5 S / 3 W-24 F 1$ & & 680 & $680.9(12 / 3 / 94)$ & $\begin{array}{l}309-348 \\
387-426 \\
563-582 \\
621-641 \\
660-680\end{array}$ & 1,475 & $\begin{array}{r}12 / 3 / 93 \\
2 / 10 / 94 \\
12 / 15 / 94 \\
6 / 23 / 95\end{array}$ & $\begin{array}{l}68.34 \\
62.86 \\
69.21 \\
92.40\end{array}$ & $\begin{array}{l}1,406.66 \\
1,412.14 \\
1,405.79 \\
1,382.60\end{array}$ & $\begin{array}{l}\text { USGS } \\
\text { USGS } \\
\text { USGS } \\
\text { USGS }\end{array}$ \\
\hline $5 S / 3 W-24 F 2$ & & 729 & $691(12 / 3 / 93)$ & $686-691$ & 1,475 & $\begin{array}{r}12 / 3 / 93 \\
2 / 10 / 94 \\
11 / 18 / 94 \\
12 / 15 / 94 \\
6 / 23 / 95 \\
9 / 13 / 95\end{array}$ & $\begin{array}{l}64.22 \\
66.58 \\
76.47 \\
69.25 \\
65.77 \\
75.11\end{array}$ & $\begin{array}{l}1,410.78 \\
1,408.42 \\
1,398.53 \\
1,405.75 \\
1,409.23 \\
1,399.89\end{array}$ & $\begin{array}{l}\text { USGS } \\
\text { USGS } \\
\text { USGS } \\
\text { USGS } \\
\text { USGS } \\
\text { USGS }\end{array}$ \\
\hline $5 S / 3 W-24 F 3$ & & 729 & $403.8(12 / 3 / 93)$ & $399-404$ & 1,475 & $\begin{array}{r}12 / 3 / 93 \\
2 / 10 / 94 \\
11 / 18 / 94 \\
12 / 15 / 94 \\
6 / 23 / 95 \\
9 / 13 / 95\end{array}$ & $\begin{array}{r}69.90 \\
68.21 \\
83.52 \\
72.76 \\
108.69 \\
99.99\end{array}$ & $\begin{array}{l}1,405.10 \\
1,406.79 \\
1,391.48 \\
1,402.24 \\
1,366.31 \\
1,375.01\end{array}$ & $\begin{array}{l}\text { USGS } \\
\text { USGS } \\
\text { USGS } \\
\text { USGS } \\
\text { USGS } \\
\text { USGS }\end{array}$ \\
\hline $5 S / 3 W-24 F 4$ & & 729 & $155.3(12 / 3 / 93)$ & $150-155$ & 1,475 & $\begin{array}{r}12 / 3 / 93 \\
2 / 10 / 94 \\
11 / 18 / 94 \\
12 / 15 / 94 \\
6 / 23 / 95 \\
9 / 13 / 95\end{array}$ & $\begin{array}{l}58.06 \\
57.40 \\
57.44 \\
57.06 \\
57.00 \\
56.86\end{array}$ & $\begin{array}{l}1,416.94 \\
1,417.60 \\
1,417.56 \\
1,417.94 \\
1,418.00 \\
1,418.14\end{array}$ & $\begin{array}{l}\text { USGS } \\
\text { USGS } \\
\text { USGS } \\
\text { USGS } \\
\text { USGS } \\
\text { USGS }\end{array}$ \\
\hline $5 S / 3 W-25 G 1$ & & - & -- & -- & 1,453 & $4 / 9 / 93$ & 34.3 & $1,418.7$ & EMWD \\
\hline $5 S / 3 W-25 K 1$ & & - & -- & - & 1,445 & $\begin{array}{r}12 / 30 / 92 \\
5 / 13 / 93 \\
12 / 30 / 93 \\
2 / 10 / 94 \\
6 / 16 / 94 \\
11 / 17 / 94 \\
6 / 23 / 95 \\
9 / 13 / 95\end{array}$ & $\begin{array}{l}27.40 \\
18.05 \\
27.40 \\
18.78 \\
19.18 \\
21.37 \\
16.50 \\
18.81\end{array}$ & $\begin{array}{l}1,417.60 \\
1,426.95 \\
1,417.60 \\
1,426.22 \\
1,425.82 \\
1,423.63 \\
1,428.50 \\
1,426.19\end{array}$ & $\begin{array}{l}\text { USGS } \\
\text { USGS } \\
\text { USGS } \\
\text { USGS } \\
\text { USGS } \\
\text { USGS } \\
\text { USGS } \\
\text { USGS }\end{array}$ \\
\hline $5 S / 3 W-28 M 1$ & MC1-430' & 445 & $430(8 / 21 / 90)$ & $425-430$ & 1,420 & $\begin{array}{r}1 / 31 / 91 \\
4 / 5 / 91 \\
6 / 13 / 91 \\
6 / 14 / 91 \\
8 / 30 / 91 \\
1 / 30 / 92 \\
1 / 31 / 92 \\
6 / 11 / 92 \\
8 / 19 / 92 \\
8 / 28 / 92 \\
4 / 24 / 95\end{array}$ & $\begin{array}{l}72.89 \\
71.15 \\
69.08 \\
69.18 \\
69.13 \\
67.83 \\
67.86 \\
63.95 \\
62.79 \\
62.71 \\
42.96\end{array}$ & $\begin{array}{l}1,347.11 \\
1,348.85 \\
1,350.92 \\
1,350.82 \\
1,350.87 \\
1,352.17 \\
1,352.14 \\
1,356.05 \\
1,357.21 \\
1,357.29 \\
1,377.04\end{array}$ & $\begin{array}{l}\text { USGS } \\
\text { USGS } \\
\text { USGS } \\
\text { USGS } \\
\text { USGS } \\
\text { USGS } \\
\text { USGS } \\
\text { USGS } \\
\text { USGS } \\
\text { USGS } \\
\text { USGS }\end{array}$ \\
\hline $5 S / 3 W-28 M 2$ & MC1-370' & 445 & $370(8 / 21 / 90)$ & $365-370$ & 1,420 & $\begin{array}{r}1 / 31 / 91 \\
4 / 5 / 91 \\
6 / 13 / 91 \\
6 / 14 / 91\end{array}$ & $\begin{array}{l}72.52 \\
70.90 \\
68.84 \\
68.96\end{array}$ & $\begin{array}{l}1,347.48 \\
1,349.10 \\
1,351.16 \\
1,351.04\end{array}$ & $\begin{array}{l}\text { USGS } \\
\text { USGS } \\
\text { USGS } \\
\text { USGS }\end{array}$ \\
\hline
\end{tabular}


Table 3. Water-level data for selected wells in the Winchester, Menifee, and south Perris ground-water subbasins, CaliforniaContinued

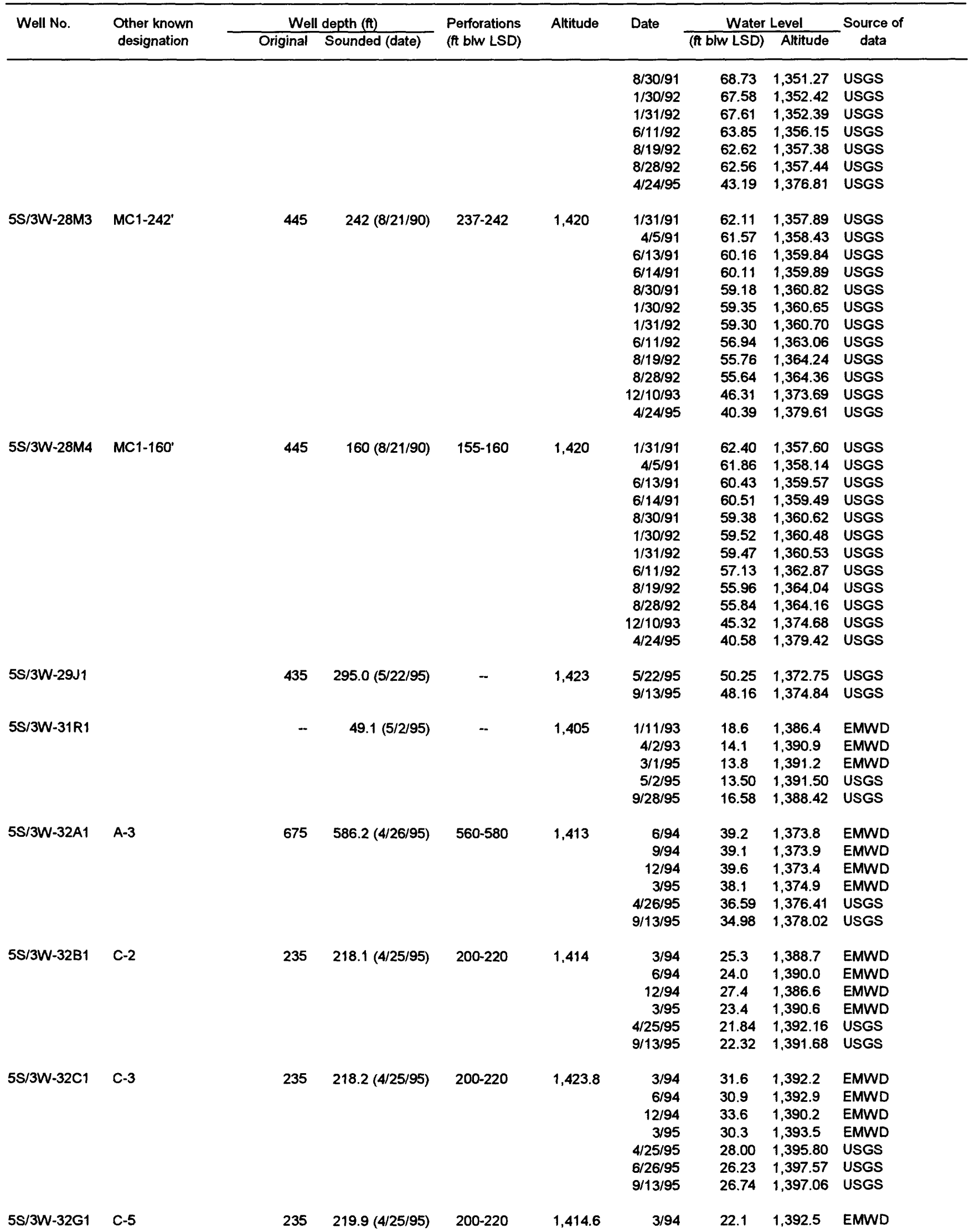


Table 3. Water-level data for selected wells in the Winchester, Menifee, and south Perris ground-water subbasins, CaliforniaContinued

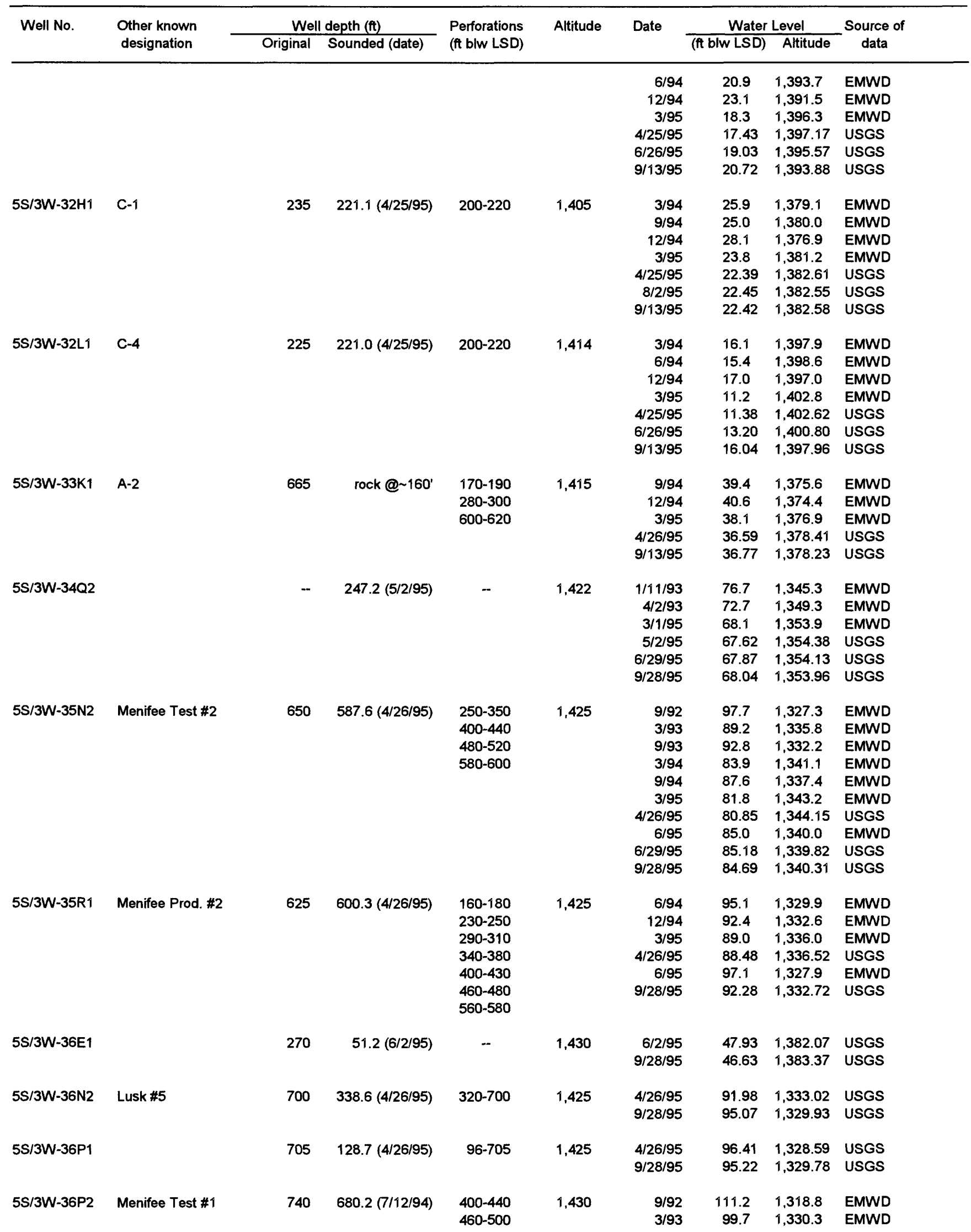


Table 3. Water-level data for selected wells in the Winchester, Menifee, and south Perris ground-water subbasins, CaliforniaContinued

\begin{tabular}{|c|c|c|c|c|c|c|c|c|c|}
\hline \multirow[t]{2}{*}{ Well No. } & \multirow{2}{*}{$\begin{array}{l}\text { Other known } \\
\text { designation }\end{array}$} & \multicolumn{2}{|c|}{ Well depth (ft) } & \multirow{2}{*}{$\begin{array}{l}\text { Perforations } \\
\text { (ft blw LSD) }\end{array}$} & \multirow[t]{2}{*}{ Altitude } & \multirow[t]{2}{*}{ Date } & \multicolumn{2}{|c|}{ Water Level } & \multirow{2}{*}{$\begin{array}{c}\text { Source of } \\
\text { data }\end{array}$} \\
\hline & & Original & Sounded (date) & & & & (ft blw LSD) & Altitude & \\
\hline & & & & $520-560$ & & $9 / 93$ & 103.2 & $1,326.8$ & EMWD \\
\hline & & & & $580-620$ & & $3 / 94$ & 91.7 & $1,338.3$ & EMWD \\
\hline & & & & $640-680$ & & $9 / 94$ & 103.2 & $1,326.8$ & EMWD \\
\hline & & & & & & $3 / 95$ & 92.1 & $1,337.9$ & EMWD \\
\hline & & & & & & $6 / 95$ & 101.5 & $1,328.5$ & EMWD \\
\hline & & & & & & $7 / 12 / 94$ & 104.71 & $1,325.29$ & USGS \\
\hline & & & & & & $9 / 28 / 95$ & 96.73 & $1,333.27$ & USGS \\
\hline $5 S / 3 W-36 Q 2$ & & - & - & - & 1,427 & $2 / 6 / 89$ & 87.60 & $1,339.40$ & EMWD \\
\hline \multirow[t]{2}{*}{ 6S/3W-1E1 } & & - & $272.7(5 / 10 / 98)$ & - & 1,428 & $5 / 10 / 95$ & 93.43 & $1,334.57$ & USGS \\
\hline & & & & & & $9 / 28 / 95$ & 96.83 & $1,331.17$ & USGS \\
\hline $6 \mathrm{~S} / 3 \mathrm{~W}-1 \mathrm{H} 1$ & & - & $369.5(6 / 2 / 95)$ & - & 1.430 & $6 / 2 / 95$ & 109.59 & $1,320.41$ & USGS \\
\hline \multirow[t]{13}{*}{$6 S / 3 W-1 J 1$} & & - & - & - & $1,429.5^{\star}$ & $8 / 16 / 68$ & $184.4^{*}$ & $1,245.1$ & RCFCD \\
\hline & & & & & & $1 / 8 / 69$ & $182.3^{*}$ & $1,247.2$ & RCFCD \\
\hline & & & & & & $8 / 6 / 69$ & $180.8^{\star}$ & $1,248.7$ & RCFCD \\
\hline & & & & & & $1 / 7 / 70$ & $178.3^{*}$ & $1,251.2$ & RCFCD \\
\hline & & & & & & $8 / 10 / 70$ & $181.5^{\star}$ & $1,248.0$ & RCFCD \\
\hline & & & & & & $1 / 11 / 71$ & $183.5^{\star}$ & $1,246.0$ & RCFCD \\
\hline & & & & & & $6 / 8 / 71$ & $179.6^{\star}$ & $1,249.9$ & RCFCD \\
\hline & & & & & & $1 / 11 / 72$ & $180.8^{\star}$ & $1,248.7$ & RCFCD \\
\hline & & & & & & $9 / 15 / 72$ & $179.7^{\star}$ & $1,249.8$ & RCFCD \\
\hline & & & & & & $1 / 16 / 73$ & $173.0^{*}$ & $1,256.5$ & RCFCD \\
\hline & & & & & & $8 / 13 / 73$ & $165.5^{\star}$ & $1,264.0$ & RCFCD \\
\hline & & & & & & $1 / 14 / 74$ & $160.0^{\star}$ & $1,269.5$ & RCFCD \\
\hline & & & & & & $6 / 12 / 74$ & $164.3^{\star}$ & $1,265.2$ & RCFCD \\
\hline \multirow[t]{2}{*}{$6 S / 3 W-1 L 1$} & & - & $109.2(5 / 10 / 95)$ & - & 1,427 & $5 / 10 / 95$ & 99.87 & $1,327.13$ & USGS \\
\hline & & & & & & $9 / 28 / 95$ & dry & - & USGS \\
\hline \multirow[t]{6}{*}{$6 S / 3 W-2 A 1$} & Menifee Prod. \#1 & 600 & $577.0(4 / 26 / 95)$ & $180-200$ & 1,425 & $1 / 94$ & 101.4 & $1,323.6$ & EMWD \\
\hline & & & & $300-360$ & & $9 / 94$ & 106.2 & $1,318.8$ & EMWD \\
\hline & & & & $380-440$ & & $3 / 95$ & 95.3 & $1,329.7$ & EMWD \\
\hline & & & & $540-560$ & & $4 / 26 / 95$ & 93.97 & $1,331.03$ & USGS \\
\hline & & & & & & $6 / 95$ & 105.2 & $1,319.8$ & EMWD \\
\hline & & & & & & $9 / 28 / 95$ & 98.27 & $1,326.73$ & USGS \\
\hline \multirow[t]{6}{*}{ 6S/3W-2D1 } & Menifee Prod. \#3 & 685 & $632.5(5 / 2 / 95)$ & $200-240$ & 1,425 & $6 / 94$ & 93.1 & $1,331.9$ & EMWD \\
\hline & & & & $280-310$ & & $12 / 94$ & 89.5 & $1,335.5$ & EMWD \\
\hline & & & & $330-350$ & & $3 / 95$ & 86.9 & $1,338.1$ & EMWD \\
\hline & & & & $410-430$ & & $5 / 2 / 95$ & 87.62 & $1,337.38$ & USGS \\
\hline & & & & $470-500$ & & $9 / 28 / 95$ & 90.27 & $1,334.73$ & USGS \\
\hline & & & & $570-610$ & & & & & \\
\hline \multirow[t]{5}{*}{$6 S / 3 W-2 E 1$} & Menifee Prod. \#4 & 695 & $651.3(4 / 26 / 95)$ & $220-300$ & 1,425 & $6 / 94$ & 98.2 & $1,326.8$ & EMWD \\
\hline & & & & $360-400$ & & $12 / 94$ & 93.6 & $1,331.4$ & EMWD \\
\hline & & & & $440-520$ & & $3 / 95$ & 90.7 & $1,334.3$ & EMWD \\
\hline & & & & $540-600$ & & $4 / 26 / 95$ & 89.79 & $1,335.21$ & USGS \\
\hline & & & & $620-640$ & & $9 / 28 / 95$ & 95.27 & $1,329.73$ & USGS \\
\hline \multirow[t]{12}{*}{$6 S / 3 W-2 F 1$} & 6S/3W-2R1** & - & - & - & 1,425 & $1 / 7 / 42$ & 46.0 & $1,379.0$ & DWR \\
\hline & & & & & & $1 / 14 / 43$ & 47.2 & $1,377.8$ & DWR \\
\hline & & & & & & $1 / 9 / 45$ & 44.2 & $1,380.8$ & DWR \\
\hline & & & & & & $1 / 10 / 46$ & 44.8 & $1,380.2$ & DWR \\
\hline & & & & & & $1 / 8 / 47$ & 46.4 & $1,378.6$ & RCFCD \\
\hline & & & & & & $1 / 19 / 48$ & 49.1 & $1,375.9$ & DWR \\
\hline & & & & & & $12 / 16 / 48$ & 52.7 & $1,372.3$ & RCFCD \\
\hline & & & & & & $1 / 13 / 50$ & 55.6 & $1,369.4$ & DWR \\
\hline & & & & & & $1 / 31 / 51$ & 52.0 & $1,373.0$ & DWR \\
\hline & & & & & & $1 / 21 / 52$ & 62.2 & $1,362.8$ & DWR \\
\hline & & & & & & $1 / 23 / 53$ & 67.5 & $1,357.5$ & DWR \\
\hline & & & & & & $12 / 20 / 53$ & 68.3 & $1,356.7$ & DWR, destroyed \\
\hline $6 S / 3 W-2 G 1$ & Menifee Lake \#2, & 625 & $\cdots$ & $100-560$ & 1,426 & $6 / 5 / 95$ & 89.81 & $1,336.19$ & USGS \\
\hline
\end{tabular}


Table 3. Water-level data for selected wells in the Winchester, Menifee, and south Perris ground-water subbasins, CaliforniaContinued

\begin{tabular}{|c|c|c|c|c|c|c|c|c|c|}
\hline \multirow[t]{2}{*}{ Well No. } & \multirow{2}{*}{$\begin{array}{l}\text { Other known } \\
\text { designation }\end{array}$} & \multicolumn{2}{|c|}{ Well depth (ft) } & \multirow{2}{*}{$\begin{array}{l}\text { Perforations } \\
\text { (ft blw LSD) }\end{array}$} & \multirow[t]{2}{*}{ Altitude } & \multirow[t]{2}{*}{ Date } & \multicolumn{2}{|c|}{ Water Level } & \multirow{2}{*}{$\begin{array}{c}\text { Source of } \\
\text { data }\end{array}$} \\
\hline & & Original & Sounded (date) & & & & (ft blw LSD) & Altitude & \\
\hline & Lusk \#2 & & & $580-620$ & & & & & \\
\hline $6 S / 3 W-2 G 2$ & $\begin{array}{l}\text { Menifee Lake \#3 } \\
\text { Lusk \#3 }\end{array}$ & 622 & - & - & 1,428 & $6 / 5 / 95$ & 92.74 & $1,335.26$ & USGS \\
\hline $6 S / 3 W-2 H 2$ & $\begin{array}{l}\text { Menifee Lake \#1, } \\
\text { Lusk \#1 }\end{array}$ & 565 & - & - & 1,428 & $6 / 2 / 95$ & 95.26 & $1,332.74$ & USGS \\
\hline $6 S / 3 W-2 J 1$ & $\begin{array}{l}\text { Menifee Lake \#4, } \\
\text { Lusk \#4 }\end{array}$ & 514 & - & - & 1,428 & 6/5/95 & 94.62 & $1,333.38$ & USGS \\
\hline \multirow[t]{5}{*}{$6 S / 3 W-3 C 1$} & & 600 & - & $370-400$ & 1,425 & $1 / 10 / 74$ & 145.0 & $1,280.0$ & RCFCD \\
\hline & & & & $430-470$ & & $3 / 5 / 75$ & 167.3 & $1,257.7$ & EMWD \\
\hline & & & & $480-490$ & & $9 / 9 / 86$ & 67.0 & $1,358.0$ & EMWD \\
\hline & & & & $530-540$ & & $3 / 21 / 91$ & 73.0 & $1,352.0$ & EMWD \\
\hline & & & & $570-580$ & & $9 / 28 / 95$ & 65.55 & $1,359.45$ & USGS \\
\hline \multirow[t]{16}{*}{$6 S / 3 W-3 H 1$} & $6 S / 3 W-3 R 1^{* *}$ & - & $100 \mathrm{R}(4 / 3 / 56)$ & - & $1,425.4$ & $1 / 19 / 48$ & 53.5 & $1,371.9$ & DWR \\
\hline & & & & & & $9 / 8 / 48$ & 60.5 & $1,364.9$ & DWR \\
\hline & & & & & & $1 / 11 / 49$ & 58.7 & $1,366.7$ & DWR \\
\hline & & & & & & $8 / 9 / 49$ & 62.2 & $1,363.2$ & DWR \\
\hline & & & & & & $1 / 13 / 50$ & 61.6 & $1,363.8$ & DWR \\
\hline & & & & & & $8 / 9 / 50$ & 67.0 & $1,358.4$ & DWR \\
\hline & & & & & & $1 / 17 / 51$ & 65.8 & 1.359 .6 & DWR \\
\hline & & & & & & $8 / 29 / 51$ & 71.9 & $1,353.5$ & DWR \\
\hline & & & & & & $4 / 1 / 52$ & 72.0 & $1,353.4$ & DWR \\
\hline & & & & & & $10 / 15 / 52$ & 72.7 & $1,352.7$ & DWR \\
\hline & & & & & & $8 / 10 / 53$ & 77.3 & $1,348.1$ & DWR \\
\hline & & & & & & $1 / 6 / 54$ & 82.1 & $1,343.3$ & DWR \\
\hline & & & & & & $7 / 7 / 54$ & 85.4 & $1,340.0$ & DWR \\
\hline & & & & & & $1 / 4 / 55$ & 90.6 & $1,334.8$ & DWR \\
\hline & & & & & & $5 / 3 / 55$ & 87.4 & $1,338.0$ & DWR \\
\hline & & & & & & $1 / 5 / 56$ & 92.0 & $1,333.4$ & DWR, destroyed \\
\hline \multirow[t]{32}{*}{$6 S / 3 W-2 F 1$} & $6 S / 3 W-3 R 3^{\star \star \star}$ & 544 & $468 \mathrm{R}$ (date unk) & -- & 1.430 & $1 / 3 / 58$ & 135.2 & $1,294.8$ & RCFCD \\
\hline & & & & & & $8 / 12 / 58$ & 144.0 & $1,286.0$ & RCFCD \\
\hline & & & & & & $3 / 4 / 59$ & 144.2 & 1.285 .8 & RCFCD \\
\hline & & & & & & $6 / 4 / 59$ & 150.1 & $1,279.9$ & RCFCD \\
\hline & & & & & & $1 / 8 / 60$ & 154.0 & $1,276.0$ & RCFCD \\
\hline & & & & & & $12 / 5 / 60$ & 157.6 & 1.272 .4 & RCFCD \\
\hline & & & & & & $11 / 2 / 61$ & 164.9 & $1,265.1$ & RCFCD \\
\hline & & & & & & $12 / 28 / 61$ & 163.7 & $1,266.3$ & RCFCD \\
\hline & & & & & & $4 / 26 / 62$ & 162.3 & $1,267.7$ & RCFCD \\
\hline & & & & & & $12 / 5 / 62$ & 168.1 & $1,261.9$ & RCFCD \\
\hline & & & & & & $3 / 27 / 63$ & 166.9 & 1.263 .1 & RCFCD \\
\hline & & & & & & $10 / 25 / 63$ & 168.4 & $1,261.6$ & RCFCD \\
\hline & & & & & & $2 / 10 / 64$ & 160.0 & $1,270.0$ & RCFCD \\
\hline & & & & & & $5 / 14 / 64$ & 163.9 & $1,266.1$ & RCFCD \\
\hline & & & & & & $11 / 9 / 64$ & 168.9 & 1.261 .1 & RCFCD \\
\hline & & & & & & $4 / 16 / 65$ & 163.5 & $1,266.5$ & RCFCD \\
\hline & & & & & & $12 / 20 / 65$ & 162.9 & $1,267.1$ & RCFCD \\
\hline & & & & & & $3 / 9 / 66$ & 158.7 & $1,271.3$ & RCFCD \\
\hline & & & & & & $12 / 15 / 66$ & 163.3 & $1,266.7$ & RCFCD \\
\hline & & & & & & $3 / 6 / 67$ & 161.3 & $1,268.7$ & RCFCD \\
\hline & & & & & & $8 / 7 / 67$ & 186.3 & 1.243 .7 & RCFCD \\
\hline & & & & & & $1 / 8 / 68$ & 164.2 & $1,265.8$ & RCFCD \\
\hline & & & & & & $6 / 11 / 68$ & 188.0 & $1,242.0$ & RCFCD \\
\hline & & & & & & $12 / 13 / 68$ & 171.4 & $1,258.6$ & RCFCD \\
\hline & & & & & & $4 / 9 / 69$ & 163.5 & $1,266.5$ & RCFCD \\
\hline & & & & & & $10 / 2 / 69$ & 171.2 & $1,258.8$ & RCFCD \\
\hline & & & & & & $3 / 10 / 70$ & 166.3 & $1,263.7$ & RCFCD \\
\hline & & & & & & $9 / 11 / 70$ & 174.3 & $1,255.7$ & RCFCD \\
\hline & & & & & & $2 / 5 / 71$ & 161.0 & $1,269.0$ & RCFCD \\
\hline & & & & & & $9 / 3 / 71$ & 178.6 & $1,251.4$ & RCFCD \\
\hline & & & & & & $1 / 7 / 72$ & 164.2 & $1,265.8$ & RCFCD \\
\hline & & & & & & $9 / 14 / 72$ & 161.0 & $1,269.0$ & RCFCD \\
\hline
\end{tabular}


Table 3. Water-level data for selected wells in the Winchester, Menifee, and south Perris ground-water subbasins, CaliforniaContinued

\begin{tabular}{|c|c|c|c|c|c|c|c|c|c|}
\hline \multirow[t]{2}{*}{ Well No. } & \multirow{2}{*}{$\begin{array}{l}\text { Other known } \\
\text { designation }\end{array}$} & \multicolumn{2}{|c|}{ Well depth (ft) } & \multirow{2}{*}{$\begin{array}{l}\text { Perforations } \\
\text { (ft blw LSD) }\end{array}$} & \multirow[t]{2}{*}{ Altitude } & \multirow[t]{2}{*}{ Date } & \multicolumn{2}{|c|}{ Water Level } & \multirow{2}{*}{$\begin{array}{c}\text { Source of } \\
\text { data }\end{array}$} \\
\hline & & Original & Sounded (date) & & & & (ft blw LSD) & Altitude & \\
\hline & & & & & & $1 / 8 / 73$ & 159.3 & $1,270.7$ & RCFCD \\
\hline & & & & & & $8 / 10 / 73$ & 148.8 & $1,281.2$ & RCFCD \\
\hline & & & & & & $1 / 3 / 74$ & 143.6 & $1,286.4$ & RCFCD \\
\hline & & & & & & $9 / 28 / 95$ & 82.20 & $1,347.80$ & USGS \\
\hline 6S/3W-3L1 & & $200 \mathrm{R}$ & - & -- & 1,460 & $6 / 5 / 95$ & 48.40 & $1,411.60$ & USGS \\
\hline \multirow[t]{4}{*}{ 6S/3W-3L.3 } & $6 S / 3 W-3 R 2^{\star \star}$ & - & - & - & 1,460 & $1 / 7 / 42$ & 47.1 & $1,412.9$ & DWR \\
\hline & & & & & & $1 / 14 / 43$ & 49.1 & $1,410.9$ & DWR \\
\hline & & & & & & $1 / 9 / 45$ & 48.1 & 1.411 .9 & DWR \\
\hline & & & & & & $1 / 10 / 46$ & 50.1 & $1,409.9$ & DWR \\
\hline \multirow[t]{12}{*}{$6 S / 3 W-4 A 1$} & $6 S / 3 W-4 R 1^{\star \star *}$ & -- & -- & -- & 1,438 & $1 / 19 / 48$ & 57.6 & $1,380.4$ & RCFCD \\
\hline & & & & & & $8 / 11 / 48$ & 63.3 & $1,374.7$ & RCFCD \\
\hline & & & & & & $1 / 11 / 49$ & 61.0 & $1,377.0$ & RCFCD \\
\hline & & & & & & $8 / 9 / 49$ & 61.4 & $1,376.6$ & RCFCD \\
\hline & & & & & & $3 / 14 / 50$ & 62.9 & $1,375.1$ & RCFCD \\
\hline & & & & & & $8 / 9 / 50$ & 65.5 & $1,372.5$ & RCFCD \\
\hline & & & & & & $1 / 17 / 51$ & 65.4 & $1,372.6$ & RCFCD \\
\hline & & & & & & $1 / 23 / 52$ & 67.2 & $1,370.8$ & DWR \\
\hline & & & & & & $10 / 15 / 52$ & 64.6 & $1,373.4$ & RCFCD \\
\hline & & & & & & $1 / 23 / 53$ & 72.6 & $1,365.4$ & DWR \\
\hline & & & & & & $10 / 16 / 53$ & 67.1 & $1,370.9$ & RCFCD \\
\hline & & & & & & $1 / 6 / 54$ & 63.3 & $1,374.7$ & DWR, abandoned \\
\hline \multirow[t]{4}{*}{$6 S / 3 W-4 K 1$} & $6 S / 3 W-4 R 2^{\star * *}$ & - & - & -- & 1,452 & $1 / 23 / 52$ & 29.2 & $1,422.8$ & DWR \\
\hline & & & & & & $1 / 23 / 53$ & 26.1 & $1,425.9$ & DWR \\
\hline & & & & & & $12 / 20 / 53$ & 27.5 & $1,424.5$ & DWR \\
\hline & & & & & & $3 / 15 / 63$ & 30.6 & 1.421 .4 & DWR \\
\hline \multirow[t]{24}{*}{$6 S / 3 W-2 F 1$} & $6 S / 3 W-5 R 1^{\star \star}$ & - & - & -- & 1,400 & $1 / 7 / 42$ & 24.3 & $1,375.9$ & DWR \\
\hline & & & & & & $1 / 14 / 43$ & 30.4 & $1,369.8$ & DWR \\
\hline & & & & & & $1 / 18 / 44$ & 28.2 & $1,372.0$ & DWR \\
\hline & & & & & & $1 / 9 / 45$ & 24.3 & $1,375.9$ & DWR \\
\hline & & & & & & $1 / 10 / 46$ & 27.7 & $1,372.5$ & DWR \\
\hline & & & & & & $1 / 8 / 47$ & 30.8 & $1,369.4$ & RCFCD \\
\hline & & & & & & $1 / 19 / 48$ & 39.2 & $1,361.0$ & RCFCD \\
\hline & & & & & & $10 / 11 / 48$ & 41.9 & $1,358.3$ & RCFCD \\
\hline & & & & & & $1 / 11 / 49$ & 43.1 & $1,357.1$ & RCFCD \\
\hline & & & & & & $5 / 11 / 49$ & 44.8 & $1,355.4$ & RCFCD \\
\hline & & & & & & $12 / 20 / 53$ & 56.3 & $1,343.9$ & RCFCD \\
\hline & & & & & & 4/10/55 & 49.7 & $1,350.5$ & RCFCD \\
\hline & & & & & & $10 / 28 / 55$ & 54.5 & $1,345.7$ & RCFCD \\
\hline & & & & & & $2 / 20 / 56$ & 56.9 & $1,343.3$ & RCFCD \\
\hline & & & & & & $10 / 15 / 56$ & 60.3 & $1,339.9$ & RCFCD \\
\hline & & & & & & $4 / 18 / 57$ & 62.1 & $1,338.1$ & RCFCD \\
\hline & & & & & & $9 / 30 / 57$ & 62.9 & $1,337.3$ & RCFCD \\
\hline & & & & & & $4 / 14 / 58$ & 52.2 & $1,348.0$ & RCFCD \\
\hline & & & & & & $11 / 10 / 58$ & 55.4 & 1.344 .8 & RCFCD \\
\hline & & & & & & $3 / 12 / 59$ & 54.4 & $1,345.8$ & RCFCD \\
\hline & & & & & & $10 / 23 / 59$ & 58.2 & $1,342.0$ & RCFCD \\
\hline & & & & & & $3 / 18 / 60$ & 58.1 & $1,342.1$ & RCFCD \\
\hline & & & & & & $10 / 24 / 60$ & 59.4 & $1,340.8$ & RCFCD \\
\hline & & & & & & $3 / 23 / 61$ & 58.0 & $1,342.2$ & RCFCD, destroyed \\
\hline $6 S / 3 S-6 A 1$ & & 144 & -- & -- & $1,400.2$ & $1 / 23 / 52$ & 54.0 & $1,346.2$ & DWR \\
\hline
\end{tabular}


Table 4. Water-level altitudes for Sun City storage pond no. 9, August-September, 1995

[Water-level altitude in feet above sea level, rounded to the nearest hundredth]

\begin{tabular}{|c|c|c|c|c|c|c|c|c|}
\hline Date & $\begin{array}{c}\text { Time } \\
\text { (24 hour) }\end{array}$ & $\begin{array}{l}\text { Water-level } \\
\text { altitude }\end{array}$ & Date & $\begin{array}{c}\text { Time } \\
\text { (24 hour) }\end{array}$ & $\begin{array}{l}\text { Water-level } \\
\text { altitude }\end{array}$ & Date & $\begin{array}{c}\text { Time } \\
\text { (24 hour) }\end{array}$ & $\begin{array}{c}\text { Water-level } \\
\text { altitude }\end{array}$ \\
\hline \multirow[t]{17}{*}{$8 / 3 / 95$} & 0700 & $1,396.28$ & $8 / 5 / 95$ & 1700 & $1,396.37$ & $8 / 8 / 95$ & 0300 & $1,396.42$ \\
\hline & 0800 & $1,396.28$ & & 1800 & $1,396.39$ & & 0400 & $1,396.42$ \\
\hline & 0900 & $1,396.29$ & & 1900 & $1,396.39$ & & 0500 & $1,396.42$ \\
\hline & 1000 & $1,396.29$ & & 2000 & $1,396.39$ & & 0600 & $1,396.42$ \\
\hline & 1100 & $1,396.29$ & & 2100 & $1,396.39$ & & 0700 & $1,396.41$ \\
\hline & 1200 & $1,396.29$ & & 2200 & $1,396.38$ & & 0800 & $1,396.41$ \\
\hline & 1300 & $1,396.30$ & & 2300 & $1,396.38$ & & 0900 & $1,396.42$ \\
\hline & 1400 & $1,396.31$ & $8 / 6 / 95$ & 0000 & $1,396.38$ & & 1000 & $1,396.42$ \\
\hline & 1500 & $1,396.32$ & & 0100 & $1,396.38$ & & 1100 & $1,396.42$ \\
\hline & 1600 & $1,396.32$ & & 0200 & $1,396.38$ & & 1200 & $1,396.42$ \\
\hline & 1700 & $1,396.33$ & & 0300 & $1,396.38$ & & 1300 & $1,396.41$ \\
\hline & 1800 & $1,396.32$ & & 0400 & $1,396.39$ & & 1400 & $1,396.42$ \\
\hline & 1900 & $1,396.32$ & & 0500 & $1,396.39$ & & 1500 & $1,396.40$ \\
\hline & 2000 & $1,396.32$ & & 0600 & $1,396.39$ & & 1600 & $1,396.46$ \\
\hline & 2100 & $1,396.31$ & & 0700 & $1,396.39$ & & 1700 & $1,396.44$ \\
\hline & 2200 & $1,396.32$ & & 0800 & $1,396.38$ & & 1800 & $1,396.45$ \\
\hline & 2300 & $1,396.32$ & & 0900 & $1,396.39$ & & 1900 & $1,396.42$ \\
\hline \multirow[t]{24}{*}{$8 / 4 / 95$} & 0000 & $1,396.32$ & & 1000 & $1,396.39$ & & 2000 & $1,396.42$ \\
\hline & 0100 & $1,396.33$ & & 1100 & $1,396.39$ & & 2100 & $1,396.42$ \\
\hline & 0200 & $1,396.33$ & & 1200 & $1,396.39$ & & 2200 & $1,396.42$ \\
\hline & 0300 & $1,396.33$ & & 1300 & $1,396.40$ & & 2300 & $1,396.42$ \\
\hline & 0400 & $1,396.33$ & & 1400 & $1,396.39$ & $8 / 9 / 95$ & 0000 & $1,396.42$ \\
\hline & 0500 & $1,396.33$ & & 1500 & $1,396.40$ & & 0100 & $1,396.41$ \\
\hline & 0600 & $1,396.33$ & & 1600 & $1,396.40$ & & 0200 & $1,396.41$ \\
\hline & 0700 & $1,396.33$ & & 1700 & $1,396.40$ & & 0300 & $1,396.41$ \\
\hline & 0800 & $1,396.33$ & & 1800 & $1,396.40$ & & 0400 & $1,396.41$ \\
\hline & 0900 & $1,396.34$ & & 1900 & $1,396.41$ & & 0500 & $1,396.41$ \\
\hline & 1000 & $1,396.33$ & & 2000 & $1,396.41$ & & 0600 & $1,396.41$ \\
\hline & 1100 & $1,396.34$ & & 2100 & $1,396.41$ & & 0700 & $1,396.41$ \\
\hline & 1200 & $1,396.36$ & & 2200 & $1,396.40$ & & 0800 & $1,396.41$ \\
\hline & 1300 & $1,396.36$ & & 2300 & $1,396.40$ & & 0900 & $1,396.41$ \\
\hline & 1400 & $1,396.36$ & $8 / 7 / 95$ & 0000 & $1,396.40$ & & 1000 & $1,396.41$ \\
\hline & 1530 & $1,396.35$ & & 0100 & $1,396.40$ & & 1100 & $1,396.41$ \\
\hline & 1600 & $1,396.37$ & & 0200 & $1,396.40$ & & 1200 & $1,396.41$ \\
\hline & 1700 & $1,396.37$ & & 0300 & $1,396.40$ & & 1300 & $1,396.41$ \\
\hline & 1800 & $1,396.37$ & & 0400 & $1,396.40$ & & 1400 & $1,396.44$ \\
\hline & 1900 & $1,396.36$ & & 0500 & $1,396.40$ & & 1500 & $1,396.43$ \\
\hline & 2000 & $1,396.37$ & & 0600 & $1,396.41$ & & 1600 & $1,396.43$ \\
\hline & 2100 & $1,396.37$ & & 0700 & $1,396.40$ & & 1700 & $1,396.41$ \\
\hline & 2200 & $1,396.37$ & & 0800 & $1,396.40$ & & 1800 & $1,396.42$ \\
\hline & 2300 & $1,396.36$ & & 0900 & $1,396.40$ & & 1900 & $1,396.40$ \\
\hline \multirow[t]{17}{*}{$8 / 5 / 95$} & 0000 & $1,396.36$ & & 1000 & $1,396.41$ & & 2000 & $1,396.40$ \\
\hline & 0100 & $1,396.36$ & & 1100 & $1,396.41$ & & 2100 & $1,396.40$ \\
\hline & 0200 & $1,396.37$ & & 1200 & $1,396.43$ & & 2200 & $1,396.40$ \\
\hline & 0300 & $1,396.36$ & & 1300 & $1,396.40$ & & 2300 & $1,396.39$ \\
\hline & 0400 & $1,396.36$ & & 1400 & $1,396.41$ & $8 / 10 / 95$ & 0000 & $1,396.39$ \\
\hline & 0500 & $1,396.37$ & & 1500 & $1,396.43$ & & 0100 & $1,396.39$ \\
\hline & 0600 & $1,396.36$ & & 1600 & $1,396.43$ & & 0200 & $1,396.39$ \\
\hline & 0700 & $1,396.37$ & & 1700 & $1,396.44$ & & 0300 & $1,396.39$ \\
\hline & 0800 & $1,396.36$ & & 1800 & $1,396.43$ & & 0400 & $1,396.39$ \\
\hline & 0900 & $1,396.37$ & & 1900 & $1,396.43$ & & 0500 & $1,396.39$ \\
\hline & 1000 & $1,396.37$ & & 2000 & $1,396.43$ & & 0600 & $1,396.39$ \\
\hline & 1100 & $1,396.37$ & & 2100 & $1,396.42$ & & 0700 & $1,396.38$ \\
\hline & 1200 & $1,396.38$ & & 2200 & $1,396.42$ & & 0800 & $1,396.39$ \\
\hline & 1300 & $1,396.37$ & & 2300 & $1,396.42$ & & 0900 & $1,396.39$ \\
\hline & 1400 & $1,396.39$ & $8 / 8 / 95$ & 0000 & $1,396.42$ & & 1000 & $1,396.39$ \\
\hline & 1500 & $1,396.37$ & & 0100 & $1,396.41$ & & 1100 & $1,396.39$ \\
\hline & 1600 & $1,396.39$ & & 0200 & $1,396.42$ & & 1200 & $1,396.40$ \\
\hline
\end{tabular}


Table 4. Water-level altitudes for Sun City storage pond no. 9, August-September, 1995--Continued

\begin{tabular}{|c|c|c|c|c|c|c|c|c|}
\hline Date & $\begin{array}{c}\text { Time } \\
\text { (24 hour) }\end{array}$ & $\begin{array}{l}\text { Water-level } \\
\text { altitude }\end{array}$ & Date & $\begin{array}{c}\text { Time } \\
\text { (24 hour) }\end{array}$ & $\begin{array}{l}\text { Water-level } \\
\text { altitude }\end{array}$ & Date & $\begin{array}{c}\text { Time } \\
\text { (24 hour) }\end{array}$ & $\begin{array}{c}\text { Water-level } \\
\text { altitude }\end{array}$ \\
\hline \multirow[t]{11}{*}{$8 / 10 / 95$} & 1300 & $1,396.41$ & $8 / 12 / 95$ & 2300 & $1,396.42$ & $8 / 15 / 95$ & 0900 & $1,396.49$ \\
\hline & 1400 & $1,396.39$ & $8 / 13 / 95$ & 0000 & $1,396.42$ & & 1000 & $1,396.50$ \\
\hline & 1500 & $1,396.40$ & & 0100 & $1,396.42$ & & 1100 & $1,396.50$ \\
\hline & 1600 & $1,396.40$ & & 0200 & $1,396.42$ & & 1200 & $1,396.49$ \\
\hline & 1700 & $1,396.40$ & & 0300 & $1,396.43$ & & 1300 & $1,396.49$ \\
\hline & 1800 & $1,396.39$ & & 0400 & $1,396.43$ & & 1400 & $1,396.50$ \\
\hline & 1900 & $1,396.39$ & & 0500 & $1,396.43$ & & 1500 & $1,396.48$ \\
\hline & 2000 & $1,396.39$ & & 0600 & $1,396.44$ & & 1600 & $1,396.49$ \\
\hline & 2100 & $1,396.39$ & & 0700 & $1,396.44$ & & 1700 & $1,396.50$ \\
\hline & 2200 & $1,396.39$ & & 0800 & $1,396.43$ & & 1800 & $1,396.49$ \\
\hline & 2300 & $1,396.39$ & & 0900 & $1,396.44$ & & 1900 & $1,396.50$ \\
\hline \multirow[t]{24}{*}{$8 / 11 / 95$} & 0000 & $1,396.40$ & & 1000 & $1,396.44$ & & 2000 & $1,396.50$ \\
\hline & 0100 & $1,396.39$ & & 1100 & $1,396.44$ & & 2100 & $1,396.50$ \\
\hline & 0200 & $1,396.39$ & & 1200 & $1,396.44$ & & 2200 & $1,396.50$ \\
\hline & 0300 & $1,396.39$ & & 1300 & $1,396.44$ & & 2300 & $1,396.50$ \\
\hline & 0400 & $1,396.39$ & & 1400 & $1,396.45$ & $8 / 16 / 95$ & 0000 & $1,396.50$ \\
\hline & 0500 & $1,396.39$ & & 1500 & $1,396.45$ & & 0100 & $1,396.50$ \\
\hline & 0600 & $1,396.40$ & & 1600 & $1,396.45$ & & 0200 & $1,396.50$ \\
\hline & 0700 & $1,396.40$ & & 1700 & $1,396.46$ & & 0300 & $1,396.51$ \\
\hline & 0800 & $1,396.39$ & & 1800 & $1,396.44$ & & 0400 & $1,396.51$ \\
\hline & 0900 & $1,396.39$ & & 1900 & $1,396.45$ & & 0500 & $1,396.52$ \\
\hline & 1000 & $1,396.39$ & & 2000 & $1,396.45$ & & 0600 & $1,396.52$ \\
\hline & 1100 & $1,396.39$ & & 2100 & $1,396.45$ & & 0700 & $1,396.53$ \\
\hline & 1200 & $1,396.39$ & & 2200 & $1,396.45$ & & 0800 & $1,396.53$ \\
\hline & 1300 & $1,396.40$ & & 2300 & $1,396.44$ & & 0900 & $1,396.54$ \\
\hline & 1400 & $1,396.39$ & $8 / 14 / 95$ & 0000 & $1,396.45$ & & 1000 & $1,396.54$ \\
\hline & 1500 & $1,396.39$ & & 0100 & $1,396.44$ & & 1100 & $1,396.54$ \\
\hline & 1600 & $1,396.44$ & & 0200 & $1,396.45$ & & 1200 & $1,396.55$ \\
\hline & 1700 & $1,396.41$ & & 0300 & $1,396.45$ & & 1300 & $1,396.56$ \\
\hline & 1800 & $1,396.41$ & & 0400 & $1,396.45$ & & 1400 & $1,396.55$ \\
\hline & 1900 & $1,396.42$ & & 0500 & $1,396.46$ & & 1500 & $1,396.56$ \\
\hline & 2000 & $1,396.42$ & & 0600 & $1,396.46$ & & 1600 & $1,396.56$ \\
\hline & 2100 & $1,396.40$ & & 0700 & $1,396.46$ & & 1700 & $1,396.56$ \\
\hline & 2200 & $1,396.41$ & & 0800 & $1,396.46$ & & 1800 & $1,396.54$ \\
\hline & 2300 & $1,396.41$ & & 0900 & $1,396.46$ & & 1900 & $1,396.56$ \\
\hline \multirow[t]{23}{*}{$8 / 12 / 95$} & 0000 & $1,396.41$ & & 1000 & $1,396.46$ & & 2000 & $1,396.56$ \\
\hline & 0100 & $1,396.40$ & & 1100 & $1,396.47$ & & 2100 & $1,396.56$ \\
\hline & 0200 & $1,396.40$ & & 1200 & $1,396.47$ & & 2200 & $1,396.57$ \\
\hline & 0300 & $1,396.40$ & & 1300 & $1,396.47$ & & 2300 & $1,396.58$ \\
\hline & 0400 & $1,396.41$ & & 1400 & $1,396.47$ & $8 / 17 / 95$ & 0000 & $1,396.58$ \\
\hline & 0500 & $1,396.41$ & & 1500 & $1,396.46$ & & 0100 & $1,396.58$ \\
\hline & 0600 & $1,396.41$ & & 1600 & $1,396.46$ & & 0200 & $1,396.59$ \\
\hline & 0700 & $1,396.41$ & & 1700 & $1,396.47$ & & 0300 & $1,396.60$ \\
\hline & 0800 & $1,396.40$ & & 1800 & $1,396.47$ & & 0400 & $1,396.60$ \\
\hline & 0900 & $1,396.40$ & & 1900 & $1,396.48$ & & 0500 & $1,396.61$ \\
\hline & 1000 & $1,396.42$ & & 2000 & $1,396.47$ & & 0600 & $1,396.61$ \\
\hline & 1100 & $1,396.41$ & & 2100 & $1,396.47$ & & 0700 & $1,396.61$ \\
\hline & 1200 & $1,396.41$ & & 2200 & $1,396.47$ & & 0800 & $1,396.61$ \\
\hline & 1300 & $1,396.41$ & & 2300 & $1,396.47$ & & 0900 & $1,396.62$ \\
\hline & 1400 & $1,396.43$ & $8 / 15 / 95$ & 0000 & $1,396.48$ & & 1000 & $1,396.62$ \\
\hline & 1500 & $1,396.41$ & & 0100 & $1,396.47$ & & 1100 & $1,396.62$ \\
\hline & 1600 & $1,396.42$ & & 0200 & $1,396.47$ & & 1200 & $1,396.61$ \\
\hline & 1700 & $1,396.42$ & & 0300 & $1,396.48$ & & 1300 & $1,396.59$ \\
\hline & 1800 & $1,396.42$ & & 0400 & $1,396.48$ & & 1400 & $1,396.60$ \\
\hline & 1900 & $1,396.43$ & & 0500 & $1,396.48$ & & 1500 & $1,396.63$ \\
\hline & 2000 & $1,396.42$ & & 0600 & $1,396.48$ & & 1600 & $1,396.61$ \\
\hline & 2100 & $1,396.42$ & & 0700 & $1,396.49$ & & 1700 & $1,396.60$ \\
\hline & 2200 & $1,396.42$ & & 0800 & $1,396.49$ & & 1800 & $1,396.61$ \\
\hline
\end{tabular}


Table 4. Water-level altitudes for Sun City storage pond no. 9, August-September, 1995--Continued

\begin{tabular}{|c|c|c|c|c|c|c|c|c|}
\hline Date & $\begin{array}{c}\text { Time } \\
\text { (24 hour) }\end{array}$ & $\begin{array}{c}\text { Water-level } \\
\text { altitude }\end{array}$ & Date & $\begin{array}{c}\text { Time } \\
\text { (24 hour) }\end{array}$ & $\begin{array}{c}\text { Water-level } \\
\text { altitude }\end{array}$ & Date & $\begin{array}{c}\text { Time } \\
\text { (24 hour) }\end{array}$ & $\begin{array}{l}\text { Water-leve } \\
\text { altitude }\end{array}$ \\
\hline \multirow[t]{5}{*}{$8 / 17 / 95$} & 1900 & $1,396.59$ & $8 / 20 / 95$ & 0500 & $1,396.78$ & $8 / 22 / 95$ & 1500 & $1,396.88$ \\
\hline & 2000 & $1,396.58$ & & 0600 & $1,396.78$ & & 1600 & $1,396.86$ \\
\hline & 2100 & $1,396.59$ & & 0700 & $1,396.78$ & & 1700 & $1,396.87$ \\
\hline & 2200 & $1,396.60$ & & 0800 & $1,396.78$ & & 1800 & $1,396.85$ \\
\hline & 2300 & $1,396.60$ & & 0900 & $1,396.79$ & & 1900 & $1,396.85$ \\
\hline \multirow[t]{24}{*}{ 8/18/95 } & 0000 & $1,396.62$ & & 1000 & $1,396.78$ & & 2000 & $1,396.86$ \\
\hline & 0100 & $1,396.62$ & & 1100 & $1,396.79$ & & 2100 & $1,396.85$ \\
\hline & 0200 & $1,396.62$ & & 1200 & $1,396.79$ & & 2200 & $1,396.85$ \\
\hline & 0300 & $1,396.63$ & & 1300 & $1,396.80$ & & 2300 & $1,396.85$ \\
\hline & 0400 & $1,396.64$ & & 1400 & $1,396.81$ & $8 / 23 / 95$ & 0000 & $1,396.85$ \\
\hline & 0500 & $1,396.64$ & & 1500 & $1,396.84$ & & 0100 & $1,396.85$ \\
\hline & 0600 & $1,396.65$ & & 1600 & $1,396.81$ & & 0200 & $1,396.85$ \\
\hline & 0700 & $1,396.65$ & & 1700 & $1,396.80$ & & 0300 & $1,396.85$ \\
\hline & 0800 & $1,396.65$ & & 1800 & $1,396.78$ & & 0400 & $1,396.86$ \\
\hline & 0900 & $1,396.66$ & & 1900 & $1,396.77$ & & 0500 & $1,396.86$ \\
\hline & 1000 & $1,396.66$ & & 2000 & $1,396.77$ & & 0600 & $1,396.85$ \\
\hline & 1100 & $1,396.67$ & & 2100 & $1,396.76$ & & 0700 & $1,396.86$ \\
\hline & 1200 & $1,396.67$ & & 2200 & $1,396.77$ & & 0800 & $1,396.85$ \\
\hline & 1300 & $1,396.67$ & & 2300 & $1,396.77$ & & 0900 & $1,396.86$ \\
\hline & 1400 & $1,396.68$ & $8 / 21 / 95$ & 0000 & $1,396.78$ & & 1000 & $1,396.86$ \\
\hline & 1500 & $1,396.68$ & & 0100 & $1,396.78$ & & 1100 & $1,396.87$ \\
\hline & 1600 & $1,396.70$ & & 0200 & $1,396.79$ & & 1200 & $1,396.85$ \\
\hline & 1700 & $1,396.70$ & & 0300 & $1,396.79$ & & 1300 & $1,396.86$ \\
\hline & 1800 & $1,396.72$ & & 0400 & $1,396.79$ & & 1400 & $1,396.85$ \\
\hline & 1900 & $1,396.72$ & & 0500 & $1,396.80$ & & 1500 & $1,396.87$ \\
\hline & 2000 & $1,396.73$ & & 0600 & $1,396.80$ & & 1600 & $1,396.87$ \\
\hline & 2100 & $1,396.72$ & & 0700 & $1,396.79$ & & 1700 & $1,396.90$ \\
\hline & 2200 & $1,396.72$ & & 0800 & $1,396.77$ & & 1800 & $1,396.87$ \\
\hline & 2300 & $1,396.72$ & & 0900 & $1,396.78$ & & 1900 & $1,396.87$ \\
\hline \multirow[t]{24}{*}{ 8/19/95 } & 0000 & $1,396.72$ & & 1000 & $1,396.79$ & & 2000 & $1,396.87$ \\
\hline & 0100 & $1,396.73$ & & 1100 & $1,396.79$ & & 2100 & $1,396.86$ \\
\hline & 0200 & $1,396.73$ & & 1200 & $1,396.81$ & & 2200 & $1,396.86$ \\
\hline & 0300 & $1,396.73$ & & 1300 & $1,396.80$ & & 2300 & $1,396.86$ \\
\hline & 0400 & $1,396.73$ & & 1400 & $1,396.81$ & $8 / 24 / 95$ & 0000 & $1,396.86$ \\
\hline & 0500 & $1,396.73$ & & 1500 & $1,396.83$ & & 0100 & $1,396.86$ \\
\hline & 0600 & $1,396.73$ & & 1600 & $1,396.83$ & & 0200 & $1,396.86$ \\
\hline & 0700 & $1,396.74$ & & 1700 & $1,396.82$ & & 0300 & $1,396.86$ \\
\hline & 0800 & $1,396.73$ & & 1800 & $1,396.82$ & & 0400 & $1,396.86$ \\
\hline & 0900 & $1,396.74$ & & 1900 & $1,396.83$ & & 0500 & $1,396.86$ \\
\hline & 1000 & $1,396.74$ & & 2000 & $1,396.83$ & & 0600 & $1,396.86$ \\
\hline & 1100 & $1,396.74$ & & 2100 & $1,396.83$ & & 0700 & $1,396.86$ \\
\hline & 1200 & $1,396.75$ & & 2200 & $1,396.83$ & & 0800 & $1,396.86$ \\
\hline & 1300 & $1,396.76$ & & 2300 & $1,396.83$ & & 0900 & $1,396.86$ \\
\hline & 1400 & $1,396.75$ & $8 / 22 / 95$ & 0000 & $1,396.83$ & & 1000 & $1,396.86$ \\
\hline & 1500 & $1,396.76$ & & 0100 & $1,396.83$ & & 1100 & $1,396.86$ \\
\hline & 1600 & $1,396.76$ & & 0200 & $1,396.83$ & & 1200 & $1,396.87$ \\
\hline & 1700 & $1,396.77$ & & 0300 & $1,396.83$ & & 1300 & $1,396.87$ \\
\hline & 1800 & $1,396.77$ & & 0400 & $1,396.83$ & & 1400 & $1,396.87$ \\
\hline & 1900 & $1,396.78$ & & 0500 & $1,396.84$ & & 1500 & $1,396.87$ \\
\hline & 2000 & $1,396.78$ & & 0600 & $1,396.84$ & & 1600 & $1,396.88$ \\
\hline & 2100 & $1,396.78$ & & 0700 & $1,396.84$ & & 1700 & $1,396.86$ \\
\hline & 2200 & $1,396.77$ & & 0800 & $1,396.84$ & & 1800 & $1,396.87$ \\
\hline & 2300 & $1,396.78$ & & 0900 & $1,396.84$ & & 1900 & $1,396.87$ \\
\hline \multirow[t]{5}{*}{$8 / 20 / 95$} & 0000 & $1,396.78$ & & 1000 & $1,396.84$ & & 2000 & $1,396.87$ \\
\hline & 0100 & $1,396.78$ & & 1100 & $1,396.84$ & & 2100 & $1,396.87$ \\
\hline & 0200 & $1,396.78$ & & 1200 & $1,396.84$ & & 2200 & $1,396.87$ \\
\hline & 0300 & $1,396.78$ & & 1300 & $1,396.85$ & & 2300 & $1,396.87$ \\
\hline & 0400 & $1,396.79$ & & 1400 & $1,396.85$ & $8 / 25 / 95$ & 0000 & $1,396.87$ \\
\hline
\end{tabular}


Tab/e 4. Water-level altitudes for Sun City storage pond no. 9, August-September, 1995--Continued

\begin{tabular}{|c|c|c|c|c|c|c|c|c|}
\hline Date & $\begin{array}{c}\text { Time } \\
\text { (24 hour) }\end{array}$ & $\begin{array}{c}\text { Water-level } \\
\text { altitude }\end{array}$ & Date & $\begin{array}{c}\text { Time } \\
\text { (24 hour) }\end{array}$ & $\begin{array}{c}\text { Water-level } \\
\text { altitude }\end{array}$ & Date & $\begin{array}{c}\text { Time } \\
\text { (24 hour) }\end{array}$ & $\begin{array}{l}\text { Water-level } \\
\text { altitude }\end{array}$ \\
\hline \multirow[t]{23}{*}{$8 / 25 / 95$} & 0100 & $1,396.87$ & $8 / 27 / 95$ & 1100 & $1,396.84$ & $8 / 29 / 95$ & 2100 & $1,396.87$ \\
\hline & 0200 & $1,396.87$ & & 1200 & $1,396.85$ & & 2200 & $1,396.87$ \\
\hline & 0300 & $1,396.87$ & & 1300 & $1,396.85$ & & 2300 & $1,396.87$ \\
\hline & 0400 & $1,396.87$ & & 1400 & $1,396.84$ & $8 / 30 / 95$ & 0000 & $1,396.87$ \\
\hline & 0500 & $1,396.87$ & & 1500 & $1,396.86$ & & 0100 & $1,396.86$ \\
\hline & 0600 & $1,396.87$ & & 1600 & $1,396.85$ & & 0200 & $1,396.86$ \\
\hline & 0700 & $1,396.87$ & & 1700 & $1,396.87$ & & 0300 & $1,396.87$ \\
\hline & 0800 & $1,396.86$ & & 1800 & $1,396.86$ & & 0400 & $1,396.87$ \\
\hline & 0900 & $1,396.86$ & & 1900 & $1,396.87$ & & 0500 & $1,396.87$ \\
\hline & 1000 & $1,396.87$ & & 2000 & $1,396.86$ & & 0600 & $1,396.87$ \\
\hline & 1100 & $1,396.87$ & & 2100 & $1,396.86$ & & 0700 & $1,396.87$ \\
\hline & 1200 & $1,396.87$ & & 2200 & $1,396.85$ & & 0800 & $1,396.86$ \\
\hline & 1300 & $1,396.88$ & & 2300 & $1,396.86$ & & 0900 & $1,396.87$ \\
\hline & 1400 & $1,396.88$ & $8 / 28 / 95$ & 0000 & $1,396.86$ & & 1000 & $1,396.87$ \\
\hline & 1500 & $1,396.87$ & & 0100 & $1,396.86$ & & 1100 & $1,396.87$ \\
\hline & 1600 & $1,396.87$ & & 0200 & $1,396.86$ & & 1200 & $1,396.87$ \\
\hline & 1700 & $1,396.87$ & & 0300 & $1,396.86$ & & 1300 & $1,396.87$ \\
\hline & 1800 & $1,396.88$ & & 0400 & $1,396.86$ & & 1400 & $1,396.88$ \\
\hline & 1900 & $1,396.88$ & & 0500 & $1,396.86$ & & 1500 & $1,396.88$ \\
\hline & 2000 & $1,396.87$ & & 0600 & $1,396.86$ & & 1600 & $1,396.88$ \\
\hline & 2100 & $1,396.86$ & & 0700 & $1,396.86$ & & 1700 & $1,396.88$ \\
\hline & 2200 & $1,396.85$ & & 0800 & $1,396.85$ & & 1800 & $1,396.90$ \\
\hline & 2300 & $1,396.82$ & & 0900 & $1,396.86$ & & 1900 & $1,396.89$ \\
\hline \multirow[t]{24}{*}{$8 / 26 / 95$} & 0000 & $1,396.81$ & & 1000 & $1,396.86$ & & 2000 & $1,396.89$ \\
\hline & 0100 & $1,396.79$ & & 1100 & $1,396.85$ & & 2100 & $1,396.87$ \\
\hline & 0200 & $1,396.79$ & & 1200 & $1,396.83$ & & 2200 & $1,396.89$ \\
\hline & 0300 & $1,396.78$ & & 1300 & $1,396.80$ & & 2300 & $1,396.88$ \\
\hline & 0400 & $1,396.78$ & & 1400 & $1,396.82$ & $8 / 31 / 95$ & 0000 & $1,396.89$ \\
\hline & 0500 & $1,396.77$ & & 1500 & $1,396.84$ & & 0100 & $1,396.88$ \\
\hline & 0600 & $1,396.77$ & & 1600 & $1,396.86$ & & 0200 & $1,396.88$ \\
\hline & 0700 & $1,396.76$ & & 1700 & $1,396.88$ & & 0300 & $1,396.88$ \\
\hline & 0800 & $1,396.75$ & & 1800 & $1,396.86$ & & 0400 & $1,396.88$ \\
\hline & 0900 & $1,396.75$ & & 1900 & $1,396.86$ & & 0500 & $1,396.87$ \\
\hline & 1000 & $1,396.75$ & & 2000 & $1,396.86$ & & 0600 & $1,396.88$ \\
\hline & 1100 & $1,396.75$ & & 2100 & $1,396.86$ & $8 / 31 / 95$ & 0700 & $1,396.87$ \\
\hline & 1200 & $1,396.76$ & & 2200 & $1,396.86$ & & 0800 & $1,396.86$ \\
\hline & 1300 & $1,396.77$ & & 2300 & $1,396.86$ & & 0900 & $1,396.87$ \\
\hline & 1400 & $1,396.77$ & $8 / 29 / 95$ & 0000 & $1,396.86$ & & 1000 & $1,396.87$ \\
\hline & 1500 & $1,396.78$ & & 0100 & $1,396.85$ & & 1100 & $1,396.87$ \\
\hline & 1600 & $1,396.79$ & & 0200 & $1,396.85$ & & 1200 & $1,396.87$ \\
\hline & 1700 & $1,396.80$ & & 0300 & $1,396.86$ & & 1300 & $1,396.88$ \\
\hline & 1800 & $1,396.82$ & & 0400 & $1,396.86$ & & 1400 & $1,396.87$ \\
\hline & 1900 & $1,396.82$ & & 0500 & $1,396.86$ & & 1500 & $1,396.86$ \\
\hline & 2000 & $1,396.83$ & & 0600 & $1,396.86$ & & 1600 & $1,396.90$ \\
\hline & 2100 & $1,396.83$ & & 0700 & $1,396.86$ & & 1700 & $1,396.89$ \\
\hline & 2200 & $1,396.83$ & & 0800 & $1,396.85$ & & 1800 & $1,396.89$ \\
\hline & 2300 & $1,396.83$ & & 0900 & $1,396.86$ & & 1900 & $1,396.88$ \\
\hline \multirow[t]{11}{*}{$8 / 27 / 95$} & 0000 & $1,396.83$ & & 1000 & $1,396.86$ & & 2000 & $1,396.88$ \\
\hline & 0100 & $1,396.83$ & & 1100 & $1,396.86$ & & 2100 & $1,396.87$ \\
\hline & 0200 & $1,396.83$ & & 1200 & $1,396.86$ & & 2200 & $1,396.88$ \\
\hline & 0300 & $1,396.83$ & & 1300 & $1,396.85$ & & 2300 & $1,396.87$ \\
\hline & 0400 & $1,396.84$ & & 1400 & $1,396.86$ & 9/1/95 & 0000 & $1,396.87$ \\
\hline & 0500 & $1,396.84$ & & 1500 & $1,396.85$ & & 0100 & $1,396.88$ \\
\hline & 0600 & $1,396.84$ & & 1600 & $1,396.86$ & & 0200 & $1,396.88$ \\
\hline & 0700 & $1,396.84$ & & 1700 & $1,396.89$ & & 0300 & $1,396.88$ \\
\hline & 0800 & $1,396.83$ & & 1800 & $1,396.89$ & & 0400 & $1,396.88$ \\
\hline & 0900 & $1,396.84$ & & 1900 & $1,396.88$ & & 0500 & $1,396.89$ \\
\hline & 1000 & $1,396.84$ & & 2000 & $1,396.86$ & & 0600 & $1,396.88$ \\
\hline
\end{tabular}


Table 4. Water-level altitudes for Sun City storage pond no. 9, August-September, 1995--Continued

\begin{tabular}{|c|c|c|c|c|c|c|c|c|}
\hline Date & $\begin{array}{c}\text { Time } \\
\text { (24 hour) }\end{array}$ & $\begin{array}{c}\text { Water-level } \\
\text { altitude }\end{array}$ & Date & $\begin{array}{c}\text { Time } \\
\text { (24 hour) }\end{array}$ & $\begin{array}{l}\text { Water-level } \\
\text { altitude }\end{array}$ & Date & $\begin{array}{c}\text { Time } \\
\text { (24 hour) }\end{array}$ & $\begin{array}{l}\text { Water-level } \\
\text { altitude }\end{array}$ \\
\hline \multirow[t]{17}{*}{ 9/1/95 } & 0700 & $1,396.88$ & 9/3/95 & 1700 & $1,396.92$ & 9/6/95 & 0300 & $1,396.91$ \\
\hline & 0800 & $1,396.87$ & & 1800 & $1,396.92$ & & 0400 & $1,396.91$ \\
\hline & 0900 & $1,396.88$ & & 1900 & $1,396.92$ & & 0500 & $1,396.91$ \\
\hline & 1000 & $1,396.87$ & & 2000 & $1,396.91$ & & 0600 & $1,396.91$ \\
\hline & 1100 & $1,396.87$ & & 2100 & $1,396.90$ & & 0700 & $1,396.91$ \\
\hline & 1200 & $1,396.88$ & & 2200 & $1,396.91$ & & 0800 & $1,396.91$ \\
\hline & 1300 & $1,396.88$ & & 2300 & $1,396.90$ & & 0900 & $1,396.91$ \\
\hline & 1400 & $1,396.89$ & 9/4/95 & 0000 & $1,396.90$ & & 1000 & $1,396.91$ \\
\hline & 1500 & $1,396.87$ & & 0100 & $1,396.90$ & & 1100 & $1,396.92$ \\
\hline & 1600 & $1,396.87$ & & 0200 & $1,396.90$ & & 1200 & $1,396.92$ \\
\hline & 1700 & $1,396.88$ & & 0300 & $1,396.90$ & & 1300 & $1,396.91$ \\
\hline & 1800 & $1,396.88$ & & 0400 & $1,396.90$ & & 1400 & $1,396.92$ \\
\hline & 1900 & $1,396.87$ & & 0500 & $1,396.90$ & & 1500 & $1,396.93$ \\
\hline & 2000 & $1,396.88$ & & 0600 & $1,396.90$ & & 1600 & $1,396.92$ \\
\hline & 2100 & $1,396.88$ & & 0700 & $1,396.90$ & & 1700 & $1,396.91$ \\
\hline & 2200 & $1,396.88$ & & 0800 & $1,396.90$ & & 1800 & $1,396.92$ \\
\hline & 2300 & $1,396.87$ & & 0900 & $1,396.90$ & & 1900 & $1,396.94$ \\
\hline \multirow[t]{24}{*}{ 9/2/95 } & 0000 & $1,396.87$ & & 1000 & $1,396.90$ & & 2000 & $1,396.92$ \\
\hline & 0100 & $1,396.87$ & & 1100 & $1,396.90$ & & 2100 & $1,396.92$ \\
\hline & 0200 & $1,396.87$ & & 1200 & $1,396.91$ & & 2200 & $1,396.92$ \\
\hline & 0300 & $1,396.88$ & & 1300 & $1,396.91$ & & 2300 & $1,396.92$ \\
\hline & 0400 & $1,396.88$ & & 1400 & $1,396.92$ & $9 / 7 / 95$ & 0000 & $1,396.92$ \\
\hline & 0500 & $1,396.88$ & & 1500 & $1,396.90$ & & 0100 & $1,396.92$ \\
\hline & 0600 & $1,396.89$ & & 1600 & $1,396.91$ & & 0200 & $1,396.92$ \\
\hline & 0700 & $1,396.88$ & & 1700 & $1,396.91$ & & 0300 & $1,396.92$ \\
\hline & 0800 & $1,396.88$ & & 1800 & $1,396.92$ & & 0400 & $1,396.92$ \\
\hline & 0900 & $1,396.88$ & & 1900 & $1,396.92$ & & 0500 & $1,396.92$ \\
\hline & 1000 & $1,396.89$ & & 2000 & $1,396.91$ & & 0600 & $1,396.92$ \\
\hline & 1100 & $1,396.88$ & & 2100 & $1,396.91$ & & 0700 & $1,396.92$ \\
\hline & 1200 & $1,396.88$ & & 2200 & $1,396.91$ & & 0800 & $1,396.92$ \\
\hline & 1300 & $1,396.89$ & & 2300 & $1,396.91$ & & 0900 & $1,396.92$ \\
\hline & 1400 & $1,396.92$ & 9/5/95 & 0000 & $1,396.91$ & & 1000 & $1,396.92$ \\
\hline & 1500 & $1,396.89$ & & 0100 & $1,396.91$ & & 1100 & $1,396.92$ \\
\hline & 1600 & $1,396.91$ & & 0200 & $1,396.91$ & & 1200 & $1,396.92$ \\
\hline & 1700 & $1,396.87$ & & 0300 & $1,396.91$ & & 1300 & $1,396.93$ \\
\hline & 1800 & $1,396.89$ & & 0400 & $1,396.91$ & & 1400 & $1,396.90$ \\
\hline & 1900 & $1,396.89$ & & 0500 & $1,396.91$ & & 1500 & $1,396.93$ \\
\hline & 2000 & $1,396.89$ & & 0600 & $1,396.92$ & & 1600 & $1,396.90$ \\
\hline & 2100 & $1,396.89$ & & 0700 & $1,396.92$ & & 1700 & $1,396.93$ \\
\hline & 2200 & $1,396.89$ & & 0800 & $1,396.91$ & & 1800 & $1,396.95$ \\
\hline & 2300 & $1,396.90$ & & 0900 & $1,396.91$ & & 1900 & $1,396.93$ \\
\hline \multirow[t]{17}{*}{$9 / 3 / 95$} & 0000 & $1,396.89$ & & 1000 & $1,396.92$ & & 2000 & $1,396.93$ \\
\hline & 0100 & $1,396.89$ & & 1100 & $1,396.92$ & & 2100 & $1,396.92$ \\
\hline & 0200 & $1,396.89$ & & 1200 & $1,396.91$ & & 2200 & $1,396.92$ \\
\hline & 0300 & $1,396.89$ & & 1300 & $1,396.90$ & & 2300 & $1,396.92$ \\
\hline & 0400 & $1,396.90$ & & 1400 & $1,396.92$ & 9/8/95 & 0000 & $1,396.92$ \\
\hline & 0500 & $1,396.89$ & & 1500 & $1,396.92$ & & 0100 & $1,396.92$ \\
\hline & 0600 & $1,396.89$ & & 1600 & $1,396.92$ & & 0200 & $1,396.92$ \\
\hline & 0700 & $1,396.89$ & & 1700 & $1,396.91$ & & 0300 & $1,396.92$ \\
\hline & 0800 & $1,396.89$ & & 1800 & $1,396.93$ & & 0400 & $1,396.92$ \\
\hline & 0900 & $1,396.89$ & & 1900 & $1,396.92$ & & 0500 & $1,396.92$ \\
\hline & 1000 & $1,396.89$ & & 2000 & $1,396.91$ & & 0600 & $1,396.92$ \\
\hline & 1100 & $1,396.90$ & & 2100 & $1,396.91$ & & 0700 & $1,396.92$ \\
\hline & 1200 & $1,396.90$ & & 2200 & $1,396.92$ & & 0800 & $1,396.92$ \\
\hline & 1300 & $1,396.90$ & & 2300 & $1,396.91$ & & 0900 & $1,396.92$ \\
\hline & 1400 & $1,396.90$ & $9 / 6 / 95$ & 0000 & $1,396.91$ & & 1000 & $1,396.93$ \\
\hline & 1500 & $1,396.88$ & & 0100 & $1,396.91$ & & 1100 & $1,396.92$ \\
\hline & 1600 & $1,396.91$ & & 0200 & $1,396.91$ & & 1200 & $1,396.94$ \\
\hline
\end{tabular}


Table 4. Water-level altitudes for Sun City storage pond no. 9, August-September, 1995--Continued

\begin{tabular}{|c|c|c|c|c|c|c|c|c|}
\hline Date & $\begin{array}{c}\text { Time } \\
\text { (24 hour) }\end{array}$ & $\begin{array}{c}\text { Water-level } \\
\text { altitude }\end{array}$ & Date & $\begin{array}{c}\text { Time } \\
\text { (24 hour) }\end{array}$ & $\begin{array}{l}\text { Water-level } \\
\text { altitude }\end{array}$ & Date & $\begin{array}{c}\text { Time } \\
\text { (24 hour) }\end{array}$ & $\begin{array}{l}\text { Water-level } \\
\text { altitude }\end{array}$ \\
\hline \multirow[t]{11}{*}{ 9/8/95 } & 1300 & $1,396.92$ & $9 / 10 / 95$ & 1900 & $1,396.96$ & $9 / 13 / 95$ & 0100 & $1,396.95$ \\
\hline & 1400 & $1,396.93$ & & 2000 & $1,396.94$ & & 0300 & $1,396.95$ \\
\hline & 1500 & $1,396.92$ & & 2100 & $1,396.95$ & & 0400 & $1,396.95$ \\
\hline & 1600 & $1,396.90$ & & 2200 & $1,396.94$ & & 0500 & $1,396.95$ \\
\hline & 1700 & $1,396.93$ & & 2300 & $1,396.94$ & & 0600 & $1,396.95$ \\
\hline & 1800 & $1,396.96$ & $9 / 11 / 95$ & 0000 & $1,396.94$ & & 0700 & $1,396.95$ \\
\hline & 1900 & $1,396.93$ & & 0100 & $1,396.94$ & & 0800 & $1,396.95$ \\
\hline & 2000 & $1,396.93$ & & 0200 & $1,396.94$ & & 0900 & $1,396.95$ \\
\hline & 2100 & $1,396.93$ & & 0300 & $1,396.94$ & & 1000 & $1,396.95$ \\
\hline & 2200 & $1,396.93$ & & 0400 & $1,396.94$ & & 1100 & $1,396.96$ \\
\hline & 2300 & $1,396.93$ & & 0500 & $1,396.94$ & & 1200 & $1,396.96$ \\
\hline \multirow[t]{24}{*}{ 9/9/95 } & 0000 & $1,396.93$ & & 0600 & $1,396.94$ & & 1300 & $1,396.96$ \\
\hline & 0100 & $1,396.93$ & & 0700 & $1,396.94$ & & 1400 & $1,396.96$ \\
\hline & 0200 & $1,396.92$ & & 0800 & $1,396.93$ & & 1500 & $1,396.97$ \\
\hline & 0300 & $1,396.93$ & & 0900 & $1,396.94$ & & 1600 & $1,396.97$ \\
\hline & 0400 & $1,396.93$ & & 1000 & $1,396.93$ & & 1700 & $1,396.97$ \\
\hline & 0500 & $1,396.93$ & & 1100 & $1,396.94$ & & 1800 & $1,396.97$ \\
\hline & 0600 & $1,396.93$ & & 1200 & $1,396.94$ & & 1900 & $1,396.97$ \\
\hline & 0700 & $1,396.92$ & & 1300 & $1,396.94$ & & 2000 & $1,396.96$ \\
\hline & 0800 & $1,396.92$ & & 1400 & $1,396.94$ & & 2100 & $1,396.96$ \\
\hline & 0900 & $1,396.93$ & & 1500 & $1,396.95$ & & 2200 & $1,396.96$ \\
\hline & 1000 & $1,396.93$ & & 1600 & $1,396.94$ & & 2300 & $1,396.96$ \\
\hline & 1100 & $1,396.92$ & & 1700 & $1,396.96$ & $9 / 14 / 95$ & 0000 & $1,396.96$ \\
\hline & 1200 & $1,396.93$ & & 1800 & $1,396.97$ & & 0100 & $1,396.96$ \\
\hline & 1300 & $1,396.93$ & & 1900 & $1,396.96$ & & 0200 & $1,396.96$ \\
\hline & 1400 & $1,396.93$ & & 2000 & $1,396.94$ & & 0300 & $1,396.96$ \\
\hline & 1500 & $1,396.94$ & & 2100 & $1,396.95$ & & 0400 & $1,396.96$ \\
\hline & 1600 & $1,396.94$ & & 2200 & $1,396.94$ & & 0500 & $1,396.96$ \\
\hline & 1700 & $1,396.94$ & & 2300 & $1,396.95$ & & 0600 & $1,396.96$ \\
\hline & 1800 & $1,396.94$ & $9 / 12 / 95$ & 0000 & $1,396.95$ & & 0700 & $1,396.96$ \\
\hline & 1900 & $1,396.94$ & & 0100 & $1,396.95$ & & 0800 & $1,396.96$ \\
\hline & 2000 & $1,396.94$ & & 0200 & $1,396.94$ & & 0900 & $1,396.96$ \\
\hline & 2100 & $1,396.94$ & & 0300 & $1,396.95$ & & 1000 & $1,396.96$ \\
\hline & 2200 & $1,396.93$ & & 0400 & $1,396.94$ & & 1100 & $1,396.96$ \\
\hline & 2300 & $1,396.93$ & & 0500 & $1,396.95$ & & 1200 & $1,396.97$ \\
\hline \multirow[t]{19}{*}{$9 / 10 / 95$} & 0000 & $1,396.93$ & & 0600 & $1,396.95$ & & 1300 & $1,396.96$ \\
\hline & 0100 & $1,396.93$ & & 0700 & $1,396.94$ & & 1400 & $1,396.97$ \\
\hline & 0200 & $1,396.93$ & & 0800 & $1,396.94$ & & 1500 & $1,396.98$ \\
\hline & 0300 & $1,396.93$ & & 0900 & $1,396.94$ & & 1600 & $1,397.02$ \\
\hline & 0400 & $1,396.93$ & & 1000 & $1,396.94$ & & 1700 & $1,396.97$ \\
\hline & 0500 & $1,396.93$ & & 1100 & $1,396.94$ & & 1800 & $1,396.99$ \\
\hline & 0600 & $1,396.93$ & & 1200 & $1,396.94$ & & 1900 & $1,396.98$ \\
\hline & 0700 & $1,396.93$ & & 1300 & $1,396.95$ & & 2000 & $1,396.97$ \\
\hline & 0800 & $1,396.92$ & & 1400 & $1,396.95$ & & 2100 & $1,396.98$ \\
\hline & 0900 & $1,396.93$ & & 1500 & $1,396.96$ & & 2300 & $1,396.97$ \\
\hline & 1000 & $1,396.93$ & & 1600 & $1,396.96$ & $9 / 15 / 95$ & 0000 & $1,396.97$ \\
\hline & 1100 & $1,396.93$ & & 1700 & $1,396.97$ & & 0100 & $1,396.97$ \\
\hline & 1200 & $1,396.93$ & & 1800 & $1,396.95$ & & 0200 & $1,396.97$ \\
\hline & 1300 & $1,396.94$ & & 1900 & $1,396.97$ & & 0300 & $1,396.97$ \\
\hline & 1400 & $1,396.94$ & & 2000 & $1,396.95$ & & 0400 & $1,396.97$ \\
\hline & 1500 & $1,396.95$ & & 2100 & $1,396.96$ & & 0500 & $1,396.97$ \\
\hline & 1600 & $1,396.93$ & & 2200 & $1,396.95$ & & 0600 & $1,396.97$ \\
\hline & 1700 & $1,396.94$ & & 2300 & $1,396.95$ & & 0700 & $1,396.97$ \\
\hline & 1800 & $1,396.94$ & 9/13/95 & 0000 & $1,396.95$ & & 0800 & $1,396.97$ \\
\hline
\end{tabular}


Table 5. Water-level altitudes for Sun City and Winchester observation wells, April-September 1995

[Location of wells shown in figure 8 . Perforated interval for all Sun City wells except well 32A1 is 200-220 feet. Perforated interval for well 32A is 560-580 feet. Measuring points for Sun City wells were surveyed; therefore, water-level altitudes are reported to hundredths of a foot. Perforated interval for all Winchester wells is $50-70$ feet. $\mathrm{ft}$, foot; - - , no data]

\begin{tabular}{|c|c|c|c|c|c|c|c|c|c|}
\hline \multicolumn{10}{|c|}{ SUN CITY SITE } \\
\hline $\begin{array}{l}\text { Pond no. } 9 \\
\text { water-level } \\
\text { altitude (ft) }\end{array}$ & Well name & Date & $\begin{array}{l}\text { Water-level } \\
\text { altitude }(\mathrm{ft})\end{array}$ & $\begin{array}{c}\text { Water-level } \\
\text { change }\end{array}$ & & Well name & Date & $\begin{array}{l}\text { Water-level } \\
\text { altitude }(\mathrm{ft})\end{array}$ & $\begin{array}{c}\text { Water-level } \\
\text { change }\end{array}$ \\
\hline \multirow{12}{*}{$1,396.96$} & $5 S / 3 W-32 L 1$ & $4 / 25 / 95$ & $1,402.62$ & & & $5 S / 3 W-32 B 1$ & $4 / 25 / 95$ & $1,392.16$ & \\
\hline & & $6 / 26 / 95$ & $1,400.80$ & & & & $6 / 26 / 95$ & - & \\
\hline & & 9/13/95 & $1,397.96$ & & & & $9 / 13 / 95$ & $1,391.68$ & \\
\hline & & $4 / 25-9 / 13$ & & $-4.66 \mathrm{ft}$ & & & $4 / 25-9 / 13$ & & $-0.48 \mathrm{ft}$ \\
\hline & $5 S / 3 W-32 C 1$ & $4 / 25 / 95$ & $1,395.80$ & & & $5 S / 3 W-32 \mathrm{H} 1$ & $4 / 25 / 95$ & $1,382.61$ & \\
\hline & & 6/26/95 & $1,397.51$ & & & & $6 / 26 / 95$ & - - & \\
\hline & & $9 / 13 / 95$ & $1,397.06$ & & & & $9 / 13 / 95$ & $1,382.58$ & \\
\hline & & $4 / 25-9 / 13$ & & $+1.26 \mathrm{ft}$ & & & $4 / 25-9 / 13$ & & $-0.03 \mathrm{ft}$ \\
\hline & $5 S / 3 W-32 G 1$ & $4 / 25 / 95$ & $1,397.17$ & & & $5 S / 3 W-32 A 1$ & $4 / 25 / 95$ & $1,376.41$ & \\
\hline & & 6/26/95 & $1,394.37$ & & & & $6 / 26 / 95$ & -- & \\
\hline & & $9 / 13 / 95$ & $1,393.88$ & & & & $9 / 13 / 95$ & $1,378.02$ & \\
\hline & & $4 / 25-9 / 13$ & & $-3.29 \mathrm{ft}$ & & & $4 / 25-9 / 13$ & & $+1.61 \mathrm{ft}$ \\
\hline \multicolumn{10}{|c|}{ WINCHESTER SITE } \\
\hline $\begin{array}{c}\text { Pond B } \\
\text { approximate } \\
\text { water-level } \\
\text { altitude (ft) }\end{array}$ & & & & & $\begin{array}{c}\text { Pond A } \\
\text { approximate } \\
\text { water-level } \\
\text { altitude (ft) }\end{array}$ & & & & \\
\hline 1,450 & $5 \mathrm{~S} / 2 \mathrm{~W}-30 \mathrm{H} 3$ & $5 / 23 / 95$ & $1,447.9$ & & 1,455 & $5 \mathrm{~S} / 2 \mathrm{~W}-30 \mathrm{H} 2$ & $5 / 11 / 95$ & $1,449.8$ & \\
\hline 1,448 & & $6 / 195$ & $1,447.8$ & & 1,452 & & $6 / 195$ & $1,449.2$ & \\
\hline $1,449.5$ & & $7 / 6 / 95$ & $1,447.4$ & & $1,448.5$ & & $7 / 6 / 95$ & - & \\
\hline \multirow[t]{17}{*}{$1,438.5$} & & 9/11/95 & $1,440.1$ & & $1,437.5$ & & $9 / 11 / 95$ & $1,442.4$ & \\
\hline & & $5 / 23-9 / 11$ & & $-7.8 \mathrm{ft}$ & & & $5 / 11-9 / 11$ & & $-7.4 \mathrm{ft}$ \\
\hline & $5 S / 2 W-30 G 2$ & $5 / 23 / 95$ & $1,441.4$ & & & $5 \mathrm{~S} / 2 \mathrm{~W}-30 \mathrm{H} 1$ & $5 / 11 / 95$ & $1,452.9$ & \\
\hline & & 6/ 195 & $1,440.8$ & & & & $6 / / 95$ & $1,451.6$ & \\
\hline & & $7 / 7 / 95$ & $1,440.1$ & & & & $7 / 6 / 95$ & $1,449.9$ & \\
\hline & & 9/13/95 & $1,437.2$ & & & & $9 / 11 / 95$ & $1,443.0$ & \\
\hline & & $5 / 23-9 / 13$ & & $-4.2 \mathrm{ft}$ & & & $5 / 11-9 / 11$ & & $-9.9 \mathrm{ft}$ \\
\hline & $5 S / 2 W-30 G 3$ & $5 / 23 / 95$ & $1,442.0$ & & & $5 S / 2 W-30 A 1$ & $5 / 23 / 95$ & $1,456.2$ & \\
\hline & & $6 / 195$ & $1,441.5$ & & & & $6 / / 95$ & $1,454.2$ & \\
\hline & & 7/6/95 & $1,441.0$ & & & & $7 / 6 / 95$ & $1,451.9$ & \\
\hline & & 9/13/95 & $1,435.7$ & & & & $9 / 13 / 95$ & $1,444.7$ & \\
\hline & & $5 / 23-9 / 13$ & & $-6.3 \mathrm{ft}$ & & & $5 / 23-9 / 13$ & & $-11.5 \mathrm{ft}$ \\
\hline & $5 \mathrm{~S} / 2 \mathrm{~W}-30 \mathrm{~B} 2$ & $5 / 23 / 95$ & -- & & & $5 S / 2 W-30 B 1$ & 5/23/95 & $1,453.4$ & \\
\hline & & 6/2/95 & $1,446.6$ & & & & $6 / / 95$ & -- & \\
\hline & & 7/6/95 & $1,446.4$ & & & & $7 / 6 / 95$ & $1,451.8$ & \\
\hline & & 9/13/95 & $1,444.6$ & & & & $9 / 13 / 95$ & $1,447.1$ & \\
\hline & & $5 / 23-9 / 13$ & & $-2.0 \mathrm{ft}$ & & & $5 / 23-9 / 13$ & & $-6.3 \mathrm{ft}$ \\
\hline
\end{tabular}


Table 6. Conversion of water-level altitude to pond volume for Pond No. 9, Sun City Regional Water Reclamation Facility

\begin{tabular}{|c|c|c|}
\hline $\begin{array}{l}\text { Water-level } \\
\text { altitude, in } \\
\text { feet }\end{array}$ & $\begin{array}{c}\text { Volume, } \\
\text { in millions } \\
\text { of gallons }\end{array}$ & $\begin{array}{c}\text { Freeboard, } \\
\text { in feet }\end{array}$ \\
\hline 1390.0 & DRY & 20.0 \\
\hline 1390.5 & 0.8 & 19.5 \\
\hline 1391.0 & 1.5 & 19.0 \\
\hline 1391.5 & 2.3 & 18.5 \\
\hline 1392.0 & 3.0 & 18.0 \\
\hline 1392.5 & 3.8 & 17.5 \\
\hline 1393.0 & 4.5 & 17.0 \\
\hline 1393.5 & 5.4 & 16.5 \\
\hline 1394.0 & 6.2 & 16.0 \\
\hline 1393.5 & 7.0 & 15.5 \\
\hline 1395.0 & 7.8 & 15.0 \\
\hline 1395.5 & 8.7 & 14.5 \\
\hline 1396.0 & 9.6 & 14.0 \\
\hline 1396.5 & 10.5 & 13.5 \\
\hline 1397.0 & 11.3 & 13.0 \\
\hline 1397.5 & 12.2 & 12.5 \\
\hline 1398.0 & 13.0 & 12.0 \\
\hline 1398.5 & 13.9 & 11.5 \\
\hline 1399.0 & 14.8 & 11.0 \\
\hline 1399.5 & 15.7 & 10.5 \\
\hline 1400.0 & 16.6 & 10.0 \\
\hline 1400.5 & 17.7 & 9.5 \\
\hline 1401.0 & 18.4 & 9.0 \\
\hline 1401.5 & 19.4 & 8.5 \\
\hline 1402.0 & 20.4 & 8.0 \\
\hline 1402.5 & 21.6 & 7.5 \\
\hline 1403.0 & 22.5 & 7.0 \\
\hline 1403.5 & 23.7 & 6.5 \\
\hline 1404.0 & 24.8 & 6.0 \\
\hline 1404.5 & 26.1 & 5.5 \\
\hline 1405.0 & 27.3 & 5.0 \\
\hline 1405.5 & 28.5 & 4.5 \\
\hline 1406.0 & 29.7 & 4.0 \\
\hline 1406.5 & 30.9 & 3.5 \\
\hline 1407.0 & 32.1 & 3.0 \\
\hline 1407.5 & 33.4 & 2.5 \\
\hline 1408.0 & 34.7 & 2.0 \\
\hline 1408.5 & 35.8 & 1.5 \\
\hline 1409.0 & 37.0 & 1.0 \\
\hline
\end{tabular}




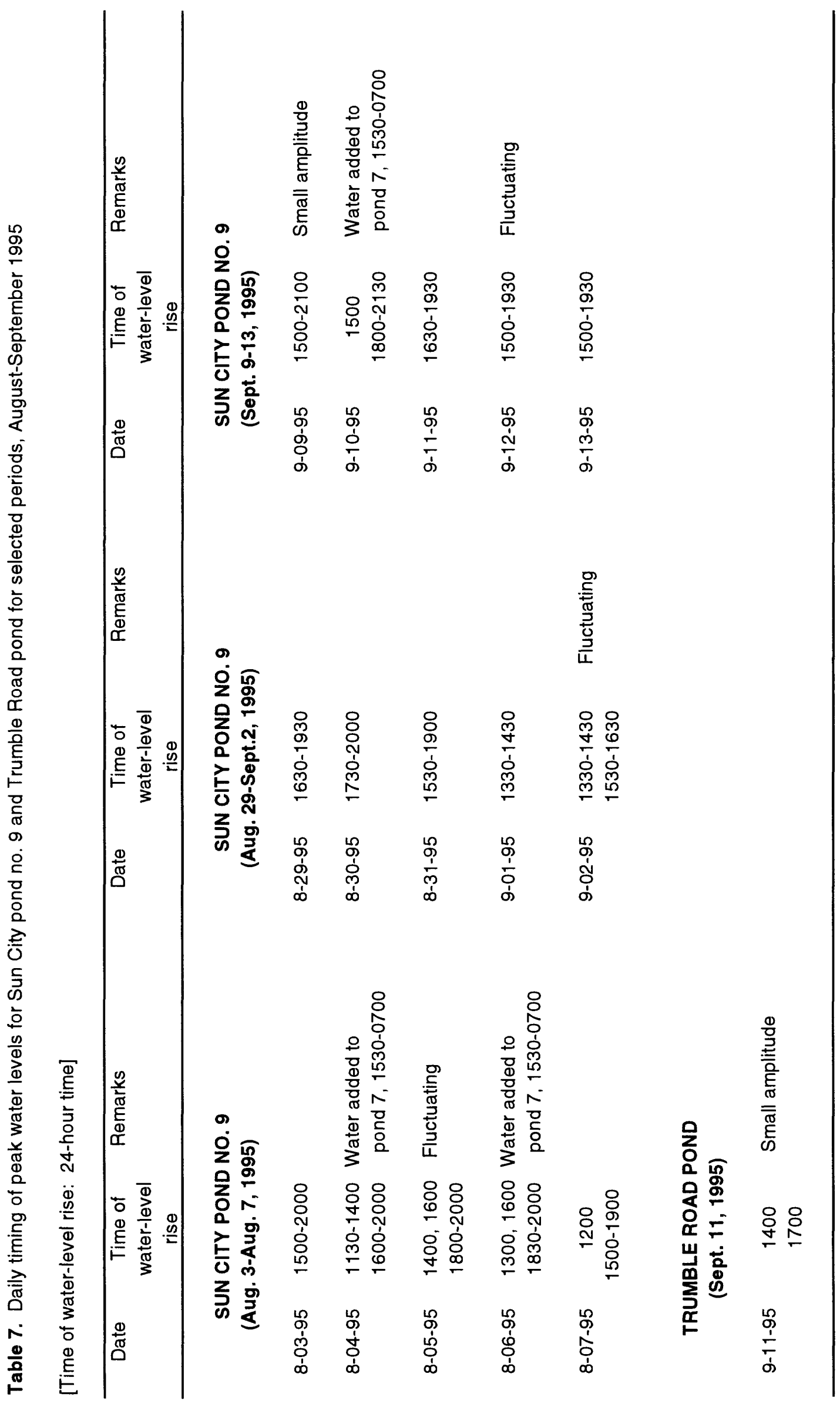


Table 8. Water-level altitudes for Trumble Road pond, July-September, 1995

[Water-level altitude in feet above sea level, rounded to the nearest hundredth]

\begin{tabular}{|c|c|c|c|c|c|c|c|c|}
\hline Date & $\begin{array}{c}\text { Time } \\
\text { (24 hour) }\end{array}$ & $\begin{array}{l}\text { Water-level } \\
\text { altitude }\end{array}$ & Date & $\begin{array}{c}\text { Time } \\
\text { (24 hour) }\end{array}$ & $\begin{array}{l}\text { Water-level } \\
\text { altitude }\end{array}$ & Date & $\begin{array}{c}\text { Time } \\
\text { (24 hour) }\end{array}$ & $\begin{array}{l}\text { Water-level } \\
\text { altitude }\end{array}$ \\
\hline \multirow[t]{3}{*}{$7 / 22 / 95$} & 2130 & $1,415.56$ & $7 / 25 / 95$ & 0600 & $1,414.89$ & $7 / 27 / 95$ & 1500 & $1,414.29$ \\
\hline & 2200 & $1,415.56$ & & 0700 & $1,414.86$ & & 1600 & $1,414.26$ \\
\hline & 2300 & $1,415.53$ & & 0800 & $1,414.87$ & & 1700 & $1,414.27$ \\
\hline \multirow[t]{24}{*}{ 7/23/95 } & 0000 & $1,415.51$ & & 0900 & $1,414.87$ & & 1800 & $1,414.25$ \\
\hline & 0115 & $1,415.49$ & & 1000 & $1,414.86$ & & 1900 & $1,414.21$ \\
\hline & 0200 & $1,415.47$ & & 1100 & $1,414.85$ & & 2000 & $1,414.21$ \\
\hline & 0300 & $1,415.46$ & & 1200 & $1,414.85$ & & 2100 & $1,414.18$ \\
\hline & 0400 & $1,415.44$ & & 1300 & $1,414.84$ & & 2200 & $1,414.17$ \\
\hline & 0500 & $1,415.43$ & & 1400 & $1,414.85$ & & 2300 & $1,414.15$ \\
\hline & 0600 & $1,415.42$ & & 1500 & $1,414.83$ & $7 / 28 / 95$ & 0000 & $1,414.13$ \\
\hline & 0700 & $1,415.40$ & & 1600 & $1,414.82$ & & 0100 & $1,414.12$ \\
\hline & 0800 & $1,415.38$ & & 1715 & $1,414.82$ & & 0200 & $1,414.10$ \\
\hline & 0900 & $1,415.37$ & & 1800 & $1,414.81$ & & 0300 & $1,414.08$ \\
\hline & 1000 & $1,415.36$ & & 1900 & $1,414.81$ & & 0400 & $1,414.07$ \\
\hline & 1100 & $1,415.35$ & & 2000 & $1,414.80$ & & 0500 & $1,414.06$ \\
\hline & 1200 & $1,415.34$ & & 2100 & $1,414.79$ & & 0600 & $1,414.04$ \\
\hline & 1300 & $1,415.33$ & & 2200 & $1,414.78$ & & 0700 & $1,414.02$ \\
\hline & 1400 & $1,415.31$ & & 2300 & $1,414.77$ & & 0800 & $1,414.01$ \\
\hline & 1500 & $1,415.30$ & $7 / 26 / 95$ & 0000 & $1,414.76$ & & 0900 & $1,414.00$ \\
\hline & 1600 & $1,415.27$ & & 0100 & $1,414.76$ & & 1000 & $1,413.99$ \\
\hline & 1700 & $1,415.27$ & & 0200 & $1,414.75$ & & 1100 & $1,413.97$ \\
\hline & 1800 & $1,415.24$ & & 0300 & $1,414.74$ & & 1200 & $1,413.98$ \\
\hline & 1900 & $1,415.24$ & & 0400 & $1,414.75$ & & 1300 & $1,413.96$ \\
\hline & 2000 & $1,415.20$ & & 0500 & $1,414.73$ & & 1400 & $1,413.96$ \\
\hline & 2100 & $1,415.21$ & & 0600 & $1,414.72$ & & 1500 & $1,413.94$ \\
\hline & 2200 & $1,415.18$ & & 0700 & $1,414.72$ & & 1600 & $1,413.92$ \\
\hline & 2300 & $1,415.17$ & & 0800 & $1,414.71$ & & 1700 & $1,413.90$ \\
\hline \multirow[t]{24}{*}{$7 / 24 / 95$} & 0000 & $1,415.16$ & & 0900 & $1,414.71$ & & 1800 & $1,413.88$ \\
\hline & 0100 & $1,415.14$ & & 1000 & $1,414.69$ & & 1900 & $1,413.86$ \\
\hline & 0200 & $1,415.13$ & & 1100 & $1,414.68$ & & 2015 & $1,413.83$ \\
\hline & 0300 & $1,415.11$ & & 1200 & $1,414.67$ & & 2100 & $1,413.83$ \\
\hline & 0400 & $1,415.10$ & & 1300 & $1,414.67$ & & 2200 & $1,413.81$ \\
\hline & 0500 & $1,415.09$ & & 1400 & $1,414.65$ & & 2300 & $1,413.78$ \\
\hline & 0600 & $1,415.07$ & & 1500 & $1,414.63$ & $7 / 29 / 95$ & 0000 & $1,413.76$ \\
\hline & 0700 & $1,415.05$ & & 1600 & $1,414.63$ & & 0100 & $1,413.75$ \\
\hline & 0800 & $1,415.04$ & & 1700 & $1,414.62$ & & 0200 & $1,413.74$ \\
\hline & 0900 & $1,415.03$ & & 1800 & $1,414.60$ & & 0300 & $1,413.72$ \\
\hline & 1000 & $1,415.02$ & & 1900 & $1,414.57$ & & 0400 & $1,413.70$ \\
\hline & 1100 & $1,415.01$ & & 2000 & $1,414.55$ & & 0500 & $1,413.68$ \\
\hline & 1200 & $1,415.00$ & & 2100 & $1,414.54$ & & 0600 & $1,413.67$ \\
\hline & 1300 & $1,414.99$ & & 2200 & $1,414.53$ & & 0700 & $1,413.65$ \\
\hline & 1400 & $1,414.99$ & & 2300 & $1,414.51$ & & 0800 & $1,413.63$ \\
\hline & 1500 & $1,414.99$ & $7 / 27 / 95$ & 0000 & $1,414.49$ & & 0900 & $1,413.62$ \\
\hline & 1600 & $1,414.98$ & & 0100 & $1,414.48$ & & 1000 & $1,413.60$ \\
\hline & 1700 & $1,414.97$ & & 0200 & $1,414.46$ & & 1100 & $1,413.58$ \\
\hline & 1800 & $1,414.96$ & & 0300 & $1,414.45$ & & 1200 & $1,413.57$ \\
\hline & 1900 & $1,414.96$ & & 0400 & $1,414.44$ & & 1300 & $1,413.56$ \\
\hline & 2000 & $1,414.94$ & & 0500 & $1,414.42$ & & 1400 & $1,413.54$ \\
\hline & 2100 & $1,414.94$ & & 0600 & $1,414.41$ & & 1500 & $1,413.53$ \\
\hline & 2200 & $1,414.94$ & & 0700 & $1,414.39$ & & 1600 & $1,413.50$ \\
\hline & 2300 & $1,414.92$ & & 0800 & $1,414.37$ & & 1700 & $1,413.50$ \\
\hline \multirow[t]{6}{*}{$7 / 25 / 95$} & 0000 & $1,414.92$ & & 0900 & $1,414.36$ & & 1800 & $1,413.47$ \\
\hline & 0100 & $1,414.91$ & & 1000 & $1,414.35$ & & 1900 & $1,413.46$ \\
\hline & 0200 & $1,414.91$ & & 1100 & $1,414.33$ & & 2000 & $1,413.44$ \\
\hline & 0300 & $1,414.90$ & & 1200 & $1,414.33$ & & 2100 & $1,413.44$ \\
\hline & 0400 & $1,414.89$ & & 1300 & $1,414.32$ & & 2200 & $1,413.42$ \\
\hline & 0500 & $1,414.89$ & & 1400 & $1,414.30$ & & 2300 & $1,413.41$ \\
\hline
\end{tabular}


Table 8. Water-level altitudes for Trumble Road pond, July-September, 1995--Continued

\begin{tabular}{|c|c|c|c|c|c|c|c|c|}
\hline Date & $\begin{array}{c}\text { Time } \\
\text { (24 hour) }\end{array}$ & $\begin{array}{c}\text { Water-level } \\
\text { altitude }\end{array}$ & Date & $\begin{array}{c}\text { Time } \\
\text { (24 hour) }\end{array}$ & $\begin{array}{c}\text { Water-level } \\
\text { altitude }\end{array}$ & Date & $\begin{array}{c}\text { Time } \\
\text { (24 hour) }\end{array}$ & $\begin{array}{c}\text { Water-level } \\
\text { altitude }\end{array}$ \\
\hline \multirow[t]{24}{*}{$7 / 30 / 95$} & 0000 & $1,413.41$ & $8 / 1 / 95$ & 0900 & $1,412.75$ & $8 / 3 / 95$ & 1800 & $1,411.94$ \\
\hline & 0100 & $1,413.41$ & & 1000 & $1,412.75$ & & 1900 & $1,411.94$ \\
\hline & 0200 & $1,413.40$ & & 1100 & $1,412.73$ & & 2000 & $1,411.92$ \\
\hline & 0300 & $1,413.40$ & & 1200 & $1,412.72$ & & 2100 & $1,411.90$ \\
\hline & 0400 & $1,413.39$ & & 1300 & $1,412.71$ & & 2200 & $1,411.88$ \\
\hline & 0500 & $1,413.39$ & & 1400 & $1,412.68$ & & 2300 & $1,411.87$ \\
\hline & 0600 & $1,413.38$ & & 1500 & $1,412.67$ & $8 / 4 / 95$ & 0000 & $1,411.86$ \\
\hline & 0700 & $1,413.37$ & & 1600 & $1,412.65$ & & 0100 & $1,411.84$ \\
\hline & 0800 & $1,413.37$ & & 1700 & $1,412.65$ & & 0200 & $1,411.83$ \\
\hline & 0900 & $1,413.37$ & & 1800 & $1,412.61$ & & 0300 & $1,411.82$ \\
\hline & 1000 & $1,413.37$ & & 1900 & $1,412.59$ & & 0400 & $1,411.81$ \\
\hline & 1100 & $1,413.36$ & & 2000 & $1,412.57$ & & 0500 & $1,411.79$ \\
\hline & 1200 & $1,413.37$ & & 2100 & $1,412.56$ & & 0600 & $1,411.78$ \\
\hline & 1300 & $1,413.35$ & & 2200 & $1,412.54$ & & 0700 & $1,411.76$ \\
\hline & 1400 & $1,413.35$ & & 2300 & $1,412.53$ & & 0800 & $1,411.75$ \\
\hline & 1500 & $1,413.34$ & $8 / 2 / 95$ & 0000 & $1,412.51$ & & 0900 & $1,411.74$ \\
\hline & 1600 & $1,413.35$ & & 0100 & $1,412.49$ & & 1000 & $1,411.73$ \\
\hline & 1700 & $1,413.32$ & & 0200 & $1,412.48$ & & 1100 & $1,411.73$ \\
\hline & 1800 & $1,413.33$ & & 0300 & $1,412.46$ & & 1145 & $1,411.70$ \\
\hline & 1900 & $1,413.29$ & & 0400 & $1,412.45$ & & 1300 & $1,411.71$ \\
\hline & 2000 & $1,413.30$ & & 0500 & $1,412.44$ & & 1400 & $1,411.70$ \\
\hline & 2100 & $1,413.28$ & & 0600 & $1,412.42$ & & 1500 & $1,411.68$ \\
\hline & 2200 & $1,413.27$ & & 0700 & $1,412.40$ & & 1600 & $1,411.65$ \\
\hline & 2300 & $1,413.26$ & & 0800 & $1,412.39$ & & 1700 & $1,411.66$ \\
\hline \multirow[t]{24}{*}{$7 / 31 / 95$} & 0000 & $1,413.26$ & & 0900 & $1,412.38$ & & 1800 & $1,411.64$ \\
\hline & 0100 & $1,413.25$ & & 1000 & $1,412.37$ & & 1900 & $1,411.63$ \\
\hline & 0200 & $1,413.25$ & & 1100 & $1,412.34$ & & 2000 & $1,411.61$ \\
\hline & 0300 & $1,413.24$ & & 1200 & $1,412.35$ & & 2100 & $1,411.59$ \\
\hline & 0400 & $1,413.23$ & & 1300 & $1,412.34$ & & 2200 & $1,411.58$ \\
\hline & 0500 & $1,413.23$ & & 1400 & $1,412.32$ & & 2300 & $1,411.56$ \\
\hline & 0600 & $1,413.22$ & & 1500 & $1,412.31$ & $8 / 5 / 95$ & 0000 & $1,411.55$ \\
\hline & 0700 & $1,413.21$ & & 1600 & $1,412.30$ & & 0100 & $1,411.55$ \\
\hline & 0800 & $1,413.20$ & & 1700 & $1,412.27$ & & 0200 & $1,411.52$ \\
\hline & 0900 & $1,413.20$ & & 1800 & $1,412.26$ & & 0300 & $1,411.52$ \\
\hline & 1000 & $1,413.19$ & & 1900 & $1,412.23$ & & 0400 & $1,411.50$ \\
\hline & 1100 & $1,413.18$ & & 2000 & $1,412.23$ & & 0500 & $1,411.49$ \\
\hline & 1200 & $1,413.18$ & & 2100 & $1,412.20$ & & 0600 & $1,411.47$ \\
\hline & 1300 & $1,413.14$ & & 2200 & $1,412.20$ & & 0700 & $1,411.44$ \\
\hline & 1400 & $1,413.12$ & & 2300 & $1,412.18$ & & 0800 & $1,411.43$ \\
\hline & 1500 & $1,413.10$ & $8 / 3 / 95$ & 0000 & $1,412.17$ & & 0900 & $1,411.44$ \\
\hline & 1600 & $1,413.07$ & & 0100 & $1,412.15$ & & 1000 & $1,411.46$ \\
\hline & 1700 & $1,413.05$ & & 0200 & $1,412.15$ & & 1100 & $1,411.44$ \\
\hline & 1800 & $1,413.03$ & & 0300 & $1,412.13$ & & 1200 & $1,411.46$ \\
\hline & 1900 & $1,413.00$ & & 0400 & $1,412.11$ & & 1300 & $1,411.44$ \\
\hline & 2000 & $1,412.97$ & & 0500 & $1,412.11$ & & 1400 & $1,411.43$ \\
\hline & 2100 & $1,412.95$ & & 0600 & $1,412.09$ & & 1500 & $1,411.43$ \\
\hline & 2200 & $1,412.93$ & & 0700 & $1,412.06$ & & 1600 & $1,411.41$ \\
\hline & 2300 & $1,412.92$ & & 0800 & $1,412.06$ & & 1700 & $1,411.39$ \\
\hline \multirow[t]{9}{*}{$8 / 1 / 95$} & 0000 & $1,412.90$ & & 0900 & $1,412.05$ & & 1800 & $1,411.38$ \\
\hline & 0100 & $1,412.88$ & & 1000 & $1,412.06$ & & 1900 & $1,411.35$ \\
\hline & 0200 & $1,412.86$ & & 1100 & $1,412.02$ & & 2000 & $1,411.33$ \\
\hline & 0300 & $1,412.85$ & & 1200 & $1,412.03$ & & 2100 & $1,411.32$ \\
\hline & 0400 & $1,412.84$ & & 1300 & $1,412.02$ & & 2200 & $1,411.29$ \\
\hline & 0500 & $1,412.82$ & & 1400 & $1,412.02$ & & 2300 & $1,411.27$ \\
\hline & 0600 & $1,412.80$ & & 1500 & $1,411.99$ & $8 / 6 / 95$ & 0000 & $1,411.27$ \\
\hline & 0700 & $1,412.78$ & & 1600 & $1,411.98$ & & 0100 & $1,411.25$ \\
\hline & 0800 & $1,412.76$ & & 1700 & $1,411.95$ & & 0200 & $1,411.24$ \\
\hline
\end{tabular}


Table 8. Water-level altitudes for Trumble Road pond, July-September, 1995--Continued

\begin{tabular}{|c|c|c|c|c|c|c|c|c|}
\hline Date & $\begin{array}{c}\text { Time } \\
\text { (24 hour) }\end{array}$ & $\begin{array}{c}\text { Water-level } \\
\text { altitude }\end{array}$ & Date & $\begin{array}{c}\text { Time } \\
\text { (24 hour) }\end{array}$ & $\begin{array}{c}\text { Water-level } \\
\text { altitude }\end{array}$ & Date & $\begin{array}{c}\text { Time } \\
\text { (24 hour) }\end{array}$ & $\begin{array}{c}\text { Water-level } \\
\text { altitude }\end{array}$ \\
\hline \multirow[t]{21}{*}{$8 / 6 / 95$} & 0300 & $1,411.23$ & $8 / 8 / 95$ & 1200 & $1,410.91$ & $8 / 10 / 95$ & 2100 & $1,409.90$ \\
\hline & 0400 & $1,411.21$ & & 1300 & $1,410.90$ & & 2200 & $1,409.87$ \\
\hline & 0500 & $1,411.20$ & & 1400 & $1,410.89$ & & 2300 & $1,409.84$ \\
\hline & 0600 & $1,411.19$ & & 1500 & $1,410,89$ & $8 / 11 / 95$ & 0000 & $1,409.82$ \\
\hline & 0700 & $1,411.16$ & & 1600 & $1,410.86$ & & 0100 & $1,409.79$ \\
\hline & 0800 & $1,411.16$ & & 1700 & $1,410,86$ & & 0200 & $1,409.77$ \\
\hline & 0900 & $1,411.15$ & & 1800 & $1,410.86$ & & 0300 & $1,409.74$ \\
\hline & 1000 & $1,411.15$ & & 1900 & $1,410.84$ & & 0400 & $1,409.72$ \\
\hline & 1100 & $1,411.16$ & & 2000 & $1,410.83$ & & 0500 & $1,409.69$ \\
\hline & 1200 & $1,411.18$ & & 2100 & $1,410.80$ & & 0600 & $1,409.67$ \\
\hline & 1300 & $1,411.18$ & & 2200 & $1,410.79$ & & 0700 & $1,409.62$ \\
\hline & 1400 & $1,411.21$ & & 2300 & $1,410.78$ & & 0800 & $1,409.59$ \\
\hline & 1500 & $1,411.20$ & $8 / 9 / 95$ & 0000 & $1,410.77$ & & 0900 & $1,409.61$ \\
\hline & 1600 & $1,411.20$ & & 0100 & $1,410.78$ & & 1000 & $1,409.56$ \\
\hline & 1700 & $1,411.18$ & & 0200 & $1,410.77$ & & 1100 & $1,409.51$ \\
\hline & 1800 & $1,411.19$ & & 0300 & $1,410.76$ & & 1200 & $1,409.55$ \\
\hline & 1900 & $1,411.20$ & & 0400 & $1,410.76$ & & 1300 & $1,409.49$ \\
\hline & 2000 & $1,411.19$ & & 0500 & $1,410.76$ & & 1400 & $1,409.47$ \\
\hline & 2100 & $1,411.18$ & & 0600 & $1,410.76$ & & 1500 & $1,409.46$ \\
\hline & 2200 & $1,411.19$ & & 0700 & $1,410.75$ & & 1600 & $1,409.43$ \\
\hline & 2300 & $1,411.18$ & & 0800 & $1,410.75$ & & 1700 & $1,409.41$ \\
\hline \multirow[t]{24}{*}{$8 / 7 / 95$} & 0000 & $1,411.18$ & & 0900 & $1,410.73$ & & 1800 & $1,409.39$ \\
\hline & 0100 & $1,411.16$ & & 1000 & $1,410.71$ & & 1900 & $1,409.36$ \\
\hline & 0200 & $1,411.17$ & & 1100 & $1,410.70$ & & 2000 & $1,409.32$ \\
\hline & 0300 & $1,411.16$ & & 1200 & $1,410.68$ & & 2100 & $1,409.28$ \\
\hline & 0400 & $1,411.16$ & & 1300 & $1,410.66$ & & 2200 & $1,409.26$ \\
\hline & 0500 & $1,411.15$ & & 1400 & $1,410.65$ & & 2300 & $1,409.23$ \\
\hline & 0600 & $1,411.15$ & & 1500 & $1,410.63$ & $8 / 12 / 95$ & 0000 & $1,409.21$ \\
\hline & 0700 & $1,411.13$ & & 1600 & $1,410.60$ & & 0100 & $1,409.19$ \\
\hline & 0800 & $1,411.12$ & & 1700 & $1,410.59$ & & 0200 & $1,409.16$ \\
\hline & 0900 & $1,411.11$ & & 1800 & $1,410.54$ & & 0300 & $1,409.13$ \\
\hline & 1000 & $1,411.12$ & & 1900 & $1,410.53$ & & 0400 & $1,409.10$ \\
\hline & 1100 & $1,411.11$ & & 2000 & $1,410.49$ & & 0500 & $1,409.08$ \\
\hline & 1200 & $1,411.13$ & & 2100 & $1,410.47$ & & 0600 & $1,409.05$ \\
\hline & 1300 & $1,411.12$ & & 2200 & $1,410.45$ & & 0700 & $1,409.01$ \\
\hline & 1400 & $1,411.11$ & & 2300 & $1,410.42$ & & 0800 & $1,409.01$ \\
\hline & 1500 & $1,411.12$ & $8 / 10 / 95$ & 0000 & $1,410.40$ & & 0900 & $1,408.96$ \\
\hline & 1600 & $1,411.11$ & & 0100 & $1,410.38$ & & 1000 & $1,408.93$ \\
\hline & 1700 & $1,411.08$ & & 0200 & $1,410.35$ & & 1100 & $1,408.91$ \\
\hline & 1800 & $1,411.07$ & & 0300 & $1,410.33$ & & 1200 & $1,408.88$ \\
\hline & 1900 & $1,411.06$ & & 0400 & $1,410.30$ & & 1300 & $1,408.87$ \\
\hline & 2000 & $1,411.06$ & & 0500 & $1,410.28$ & & 1400 & $1,408.83$ \\
\hline & 2100 & $1,411.04$ & & 0600 & $1,410.26$ & & 1500 & $1,408.83$ \\
\hline & 2200 & $1,411.03$ & & 0700 & $1,410.21$ & & 1600 & $1,408.81$ \\
\hline & 2300 & $1,411.03$ & & 0800 & $1,410.21$ & & 1700 & $1,408.78$ \\
\hline \multirow[t]{12}{*}{$8 / 8 / 95$} & 0000 & $1,411.02$ & & 0900 & $1,410.19$ & & 1800 & $1,408.73$ \\
\hline & 0100 & $1,411.01$ & & 1000 & $1,410.16$ & & 1900 & $1,408.72$ \\
\hline & 0200 & $1,411.00$ & & 1100 & $1,410.16$ & & 2000 & $1,408.68$ \\
\hline & 0300 & $1,410.99$ & & 1200 & $1,410.15$ & & 2100 & $1,408.67$ \\
\hline & 0400 & $1,410.98$ & & 1300 & $1,410.13$ & & 2200 & $1,408.64$ \\
\hline & 0500 & $1,410.97$ & & 1400 & $1,410.09$ & & 2300 & $1,408.62$ \\
\hline & 0600 & $1,410.96$ & & 1500 & $1,410.08$ & $8 / 13 / 95$ & 0000 & $1,408.58$ \\
\hline & 0700 & $1,410.94$ & & 1600 & $1,410.04$ & & 0100 & $1,408.56$ \\
\hline & 0800 & $1,410.93$ & & 1700 & $1,410.00$ & & 0200 & $1,408.53$ \\
\hline & 0900 & $1,410.93$ & & 1800 & $1,409.98$ & & 0300 & $1,408.51$ \\
\hline & 1000 & $1,410.93$ & & 1900 & $1,409.95$ & & 0400 & $1,408.49$ \\
\hline & 1100 & $1,410.95$ & & 2000 & $1,409.92$ & & 0500 & $1,408.46$ \\
\hline
\end{tabular}


Table 8. Water-level altitudes for Trumble Road pond, July-September, 1995--Continued

\begin{tabular}{|c|c|c|c|c|c|c|c|c|}
\hline Date & $\begin{array}{c}\text { Time } \\
\text { (24 hour) }\end{array}$ & $\begin{array}{c}\text { Water-level } \\
\text { altitude }\end{array}$ & Date & $\begin{array}{c}\text { Time } \\
\text { (24 hour) }\end{array}$ & $\begin{array}{c}\text { Water-level } \\
\text { altitude }\end{array}$ & Date & $\begin{array}{c}\text { Time } \\
\text { (24 hour) }\end{array}$ & $\begin{array}{c}\text { Water-level } \\
\text { altitude }\end{array}$ \\
\hline \multirow[t]{18}{*}{$8 / 13 / 95$} & 0600 & $1,408.42$ & $8 / 15 / 95$ & 1500 & $1,407.03$ & $8 / 18 / 95$ & 0000 & $1,405.66$ \\
\hline & 0700 & $1,408.39$ & & 1600 & $1,406.97$ & & 0100 & $1,405.64$ \\
\hline & 0800 & $1,408.37$ & & 1700 & $1,406.93$ & & 0200 & $1,405.61$ \\
\hline & 0900 & $1,408.34$ & & 1800 & $1,406.90$ & & 0300 & $1,405.60$ \\
\hline & 1000 & $1,408.33$ & & 1900 & $1,406.86$ & & 0400 & $1,405.56$ \\
\hline & 1100 & $1,408.33$ & & 2000 & $1,406.83$ & & 0500 & $1,405.54$ \\
\hline & 1200 & $1,408.31$ & & 2100 & $1,406.80$ & & 0600 & $1,405.52$ \\
\hline & 1300 & $1,408.28$ & & 2200 & $1,406.76$ & & 0700 & $1,405.47$ \\
\hline & 1400 & $1,408.23$ & & 2300 & $1,406.73$ & & 0800 & $1,405.45$ \\
\hline & 1500 & $1,408.21$ & $8 / 16 / 95$ & 0000 & $1,406.70$ & & 0900 & $1,405.43$ \\
\hline & 1600 & $1,408.21$ & & 0100 & $1,406.68$ & & 1000 & $1,405.43$ \\
\hline & 1700 & $1,408.15$ & & 0200 & $1,406.65$ & & 1045 & $1,405.42$ \\
\hline & 1800 & $1,408.16$ & & 0300 & $1,406.62$ & $8 / 30 / 95$ & 1130 & $1,399.60$ \\
\hline & 1900 & $1,408.12$ & & 0400 & $1,406.59$ & & 1445 & $1,399.55$ \\
\hline & 2000 & $1,408.10$ & & 0500 & $1,406.56$ & & 1545 & $1,399.54$ \\
\hline & 2100 & $1,408.08$ & & 0600 & $1,406.54$ & & 1600 & $1,399.49$ \\
\hline & 2200 & $1,408.05$ & & 0700 & $1,406.50$ & & 1700 & $1,399.48$ \\
\hline & 2300 & $1,408.02$ & & 0800 & $1,406.48$ & & 1800 & $1,399.48$ \\
\hline \multirow[t]{24}{*}{$8 / 14 / 95$} & 0000 & $1,407.99$ & & 0900 & $1,406.46$ & & 1900 & $1,399.48$ \\
\hline & 0100 & $1,407.97$ & & 1000 & $1,406.45$ & & 2000 & $1,399.46$ \\
\hline & 0200 & $1,407.94$ & & 1100 & $1,406.42$ & & 2100 & $1,399.44$ \\
\hline & 0300 & $1,407.91$ & & 1200 & $1,406.41$ & & 2200 & $1,399.44$ \\
\hline & 0400 & $1,407.88$ & & 1300 & $1,406.40$ & & 2300 & $1,399.41$ \\
\hline & 0500 & $1,407.86$ & & 1400 & $1,406.40$ & $8 / 31 / 95$ & 0000 & $1,399.40$ \\
\hline & 0600 & $1,407.82$ & & 1500 & $1,406.38$ & & 0100 & $1,399.38$ \\
\hline & 0700 & $1,407.78$ & & 1600 & $1,406.35$ & & 0200 & $1,399.37$ \\
\hline & 0800 & $1,407.77$ & & 1700 & $1,406.34$ & & 0300 & $1,399.36$ \\
\hline & 0900 & $1,407.74$ & & 1800 & $1,406.32$ & & 0400 & $1,399.34$ \\
\hline & 1000 & $1,407.75$ & & 1900 & $1,406.29$ & & 0500 & $1,399.33$ \\
\hline & 1100 & $1,407.71$ & & 2000 & $1,406.27$ & & 0600 & $1,399.31$ \\
\hline & 1200 & $1,407.71$ & & 2100 & $1,406.23$ & & 0700 & $1,399.29$ \\
\hline & 1300 & $1,407.67$ & & 2200 & $1,406.21$ & & 0800 & $1,399.28$ \\
\hline & 1400 & $1,407.65$ & & 2300 & $1,406.19$ & & 0900 & $1,399.26$ \\
\hline & 1500 & $1,407.61$ & $8 / 17 / 95$ & 0000 & $1,406.17$ & & 1000 & $1,399.24$ \\
\hline & 1600 & $1,407.60$ & & 0100 & $1,406.14$ & & 1100 & $1,399.23$ \\
\hline & 1700 & $1,407.56$ & & 0200 & $1,406.11$ & & 1200 & $1,399.22$ \\
\hline & 1800 & $1,407.54$ & & 0300 & $1,406.09$ & & 1300 & $1,399.21$ \\
\hline & 1900 & $1,407.52$ & & 0400 & $1,406.07$ & & 1400 & $1,399.19$ \\
\hline & 2000 & $1,407.50$ & & 0500 & $1,406.04$ & & 1500 & $1,399.18$ \\
\hline & 2100 & $1,407.47$ & & 0600 & $1,406.02$ & & 1600 & $1,399.16$ \\
\hline & 2200 & $1,407.44$ & & 0700 & $1,405.98$ & & 1700 & $1,399.15$ \\
\hline & 2300 & $1,407.41$ & & 0800 & $1,405.96$ & & 1800 & $1,399.14$ \\
\hline \multirow[t]{15}{*}{$8 / 15 / 95$} & 0000 & $1,407.38$ & & 0900 & $1,405.94$ & & 1900 & $1,399.11$ \\
\hline & 0100 & $1,407.35$ & & 1000 & $1,405.93$ & & 2000 & $1,399.10$ \\
\hline & 0200 & $1,407.33$ & & 1100 & $1,405.90$ & & 2100 & $1,399.09$ \\
\hline & 0300 & $1,407.32$ & & 1200 & $1,405.91$ & & 2200 & $1,399.08$ \\
\hline & 0400 & $1,407.28$ & & 1300 & $1,405.87$ & & 2300 & $1,399.06$ \\
\hline & 0500 & $1,407.26$ & & 1400 & $1,405.85$ & 9/1/95 & 0000 & $1,399.04$ \\
\hline & 0600 & $1,407.23$ & & 1500 & $1,405.84$ & & 0100 & $1,399.03$ \\
\hline & 0700 & $1,407.19$ & & 1600 & $1,405.83$ & & 0200 & $1,399.01$ \\
\hline & 0800 & $1,407.16$ & & 1700 & $1,405.81$ & & 0300 & $1,399.01$ \\
\hline & 0900 & $1,407.15$ & & 1800 & $1,405.76$ & & 0400 & $1,398.99$ \\
\hline & 1000 & $1,407.12$ & & 1900 & $1,405.77$ & & 0500 & $1,398.98$ \\
\hline & 1100 & $1,407.11$ & & 2000 & $1,405.75$ & & 0600 & $1,398.96$ \\
\hline & 1200 & $1,407.08$ & & 2100 & $1,405.71$ & & 0700 & $1,398.93$ \\
\hline & 1300 & $1,407.06$ & & 2200 & $1,405.71$ & & 0900 & $1,398.91$ \\
\hline & 1400 & $1,407.01$ & & 2300 & $1,405.68$ & & 1100 & $1,398.89$ \\
\hline
\end{tabular}


Table 8. Water-level altitudes for Trumble Road pond, July-September, 1995--Continued

\begin{tabular}{|c|c|c|c|c|c|c|c|c|}
\hline Date & $\begin{array}{c}\text { Time } \\
\text { (24 hour) }\end{array}$ & $\begin{array}{c}\text { Water-level } \\
\text { altitude }\end{array}$ & Date & $\begin{array}{c}\text { Time } \\
\text { (24 hour) }\end{array}$ & $\begin{array}{l}\text { Water-level } \\
\text { altitude }\end{array}$ & Date & $\begin{array}{c}\text { Time } \\
\text { (24 hour) }\end{array}$ & $\begin{array}{l}\text { Water-level } \\
\text { altitude }\end{array}$ \\
\hline \multirow[t]{12}{*}{ 9/1/95 } & 1200 & $1,398.88$ & 9/3/95 & 2100 & $1,397.99$ & 9/6/95 & 0600 & $1,397.09$ \\
\hline & 1300 & $1,398.87$ & & 2200 & $1,397.98$ & & 0700 & $1,397.07$ \\
\hline & 1400 & $1,398.84$ & & 2300 & $1,397.96$ & & 0800 & $1,397.03$ \\
\hline & 1500 & $1,398.82$ & $9 / 4 / 95$ & 0000 & $1,397.95$ & & 0900 & $1,397.04$ \\
\hline & 1600 & $1,398.81$ & & 0100 & $1,397.93$ & & 1000 & $1,397.02$ \\
\hline & 1700 & $1,398.80$ & & 0200 & $1,397.92$ & & 1100 & $1,397.02$ \\
\hline & 1800 & $1,398.78$ & & 0300 & $1,397.90$ & & 1200 & $1,396.98$ \\
\hline & 1900 & $1,398.76$ & & 0400 & $1,397.88$ & & 1300 & $1,396.99$ \\
\hline & 2000 & $1,398.74$ & & 0500 & $1,397.87$ & & 1400 & $1,396.96$ \\
\hline & 2100 & $1,398.74$ & & 0600 & $1,397,85$ & & 1500 & $1,396.96$ \\
\hline & 2200 & $1,398.72$ & & 0700 & $1,397.82$ & & 1600 & $1,396.93$ \\
\hline & 2300 & $1,398.71$ & & 0800 & $1,397.81$ & & 1700 & $1,396.92$ \\
\hline \multirow[t]{24}{*}{ 9/2/95 } & 0000 & $1,398.69$ & & 0900 & $1,397.79$ & & 1800 & $1,396.90$ \\
\hline & 0100 & $1,398.68$ & & 1000 & $1,397.79$ & & 1900 & $1,396.88$ \\
\hline & 0200 & $1,398.66$ & & 1100 & $1,397.77$ & & 2000 & $1,396.87$ \\
\hline & 0300 & $1,398.65$ & & 1200 & $1,397.75$ & & 2100 & $1,396.85$ \\
\hline & 0400 & $1,398.63$ & & 1300 & $1,397.73$ & & 2200 & $1,396.84$ \\
\hline & 0500 & $1,398.62$ & & 1400 & $1,397.73$ & & 2300 & $1,396.82$ \\
\hline & 0600 & $1,398.60$ & & 1500 & $1,397.71$ & 9/7/95 & 0000 & $1,396.81$ \\
\hline & 0700 & $1,398.59$ & & 1600 & $1,397.70$ & & 0100 & $1,396.79$ \\
\hline & 0800 & $1,398.56$ & & 1700 & $1,397.68$ & & 0200 & $1,396.78$ \\
\hline & 0900 & $1,398.55$ & & 1800 & $1,397.64$ & & 0300 & $1,396.76$ \\
\hline & 1000 & $1,398.53$ & & 1900 & $1,397.65$ & & 0400 & $1,396.74$ \\
\hline & 1100 & $1,398.52$ & & 2000 & $1,397.62$ & & 0500 & $1,396.73$ \\
\hline & 1200 & $1,398.51$ & & 2100 & $1,397.61$ & & 0600 & $1,396.71$ \\
\hline & 1300 & $1,398.50$ & & 2200 & $1,397.60$ & & 0700 & $1,396.70$ \\
\hline & 1400 & $1,398.47$ & & 2300 & $1,397.57$ & & 0800 & $1,396.67$ \\
\hline & 1500 & $1,398.45$ & $9 / 5 / 95$ & 0000 & $1,397.56$ & & 0900 & $1,396.63$ \\
\hline & 1600 & $1,398.44$ & & 0100 & $1,397.55$ & & 1000 & $1,396.64$ \\
\hline & 1700 & $1,398.43$ & & 0200 & $1,397.53$ & & 1100 & $1,396.61$ \\
\hline & 1800 & $1,398.42$ & & 0300 & $1,397.52$ & & 1200 & $1,396.60$ \\
\hline & 1900 & $1,398.40$ & & 0400 & $1,397.50$ & & 1300 & $1,396.60$ \\
\hline & 2000 & $1,398.40$ & & 0500 & $1,397.49$ & & 1400 & $1,396.60$ \\
\hline & 2100 & $1,398.38$ & & 0600 & $1,397.47$ & & 1500 & $1,396.56$ \\
\hline & 2200 & $1,398.36$ & & 0700 & $1,397.44$ & & 1600 & $1,396.53$ \\
\hline & 2300 & $1,398.34$ & & 0800 & $1,397.43$ & & 1700 & $1,396.51$ \\
\hline \multirow[t]{21}{*}{ 9/3/95 } & 0000 & $1,398.32$ & & 0900 & $1,397.42$ & & 1800 & $1,396.48$ \\
\hline & 0100 & $1,398.31$ & & 1000 & $1,397.40$ & & 1900 & $1,396.48$ \\
\hline & 0200 & $1,398.29$ & & 1100 & $1,397.39$ & & 2000 & $1,396.47$ \\
\hline & 0300 & $1,398.28$ & & 1200 & $1,397.39$ & & 2100 & $1,396.46$ \\
\hline & 0400 & $1,398.27$ & & 1300 & $1,397.37$ & & 2200 & $1,396.43$ \\
\hline & 0500 & $1,398.25$ & & 1400 & $1,397.34$ & & 2300 & $1,396.41$ \\
\hline & 0600 & $1,398.23$ & & 1500 & $1,397.33$ & 9/8/95 & 0000 & $1,396.40$ \\
\hline & 0700 & $1,398.21$ & & 1600 & $1,397.32$ & & 0100 & $1,396.38$ \\
\hline & 0830 & $1,398.18$ & & 1700 & $1,397.29$ & & 0200 & $1,396.36$ \\
\hline & 0900 & $1,398.17$ & & 1800 & $1,397.28$ & & 0300 & $1,396.35$ \\
\hline & 1000 & $1,398.16$ & & 1900 & $1,397.27$ & & 0400 & $1,396.33$ \\
\hline & 1100 & $1,398.15$ & & 2000 & $1,397.26$ & & 0500 & $1,396.31$ \\
\hline & 1200 & $1,398.13$ & & 2100 & $1,397.23$ & & 0600 & $1,396.30$ \\
\hline & 1300 & $1,398.12$ & & 2200 & $1,397.21$ & & 0700 & $1,396.26$ \\
\hline & 1400 & $1,398.11$ & & 2300 & $1,397.20$ & & 0800 & $1,396.24$ \\
\hline & 1500 & $1,398.10$ & $9 / 6 / 95$ & 0000 & $1,397.19$ & & 0900 & $1,396.24$ \\
\hline & 1600 & $1,398.08$ & & 0100 & $1,397.17$ & & 1000 & $1,396.23$ \\
\hline & 1700 & $1,398.05$ & & 0200 & $1,397.16$ & & 1100 & $1,396.20$ \\
\hline & 1800 & $1,398.02$ & & 0300 & $1,397.14$ & & 1200 & $1,396.19$ \\
\hline & 1900 & $1,398.03$ & & 0400 & $1,397.12$ & & 1300 & $1,396.19$ \\
\hline & 2000 & $1,398.01$ & & 0500 & $1,397.11$ & & 1400 & $1,396.15$ \\
\hline
\end{tabular}


Table 8. Water-level altitudes for Trumble Road pond, July-September, 1995--Continued

\begin{tabular}{|c|c|c|c|c|c|c|c|c|}
\hline Date & $\begin{array}{c}\text { Time } \\
\text { (24 hour) }\end{array}$ & $\begin{array}{c}\text { Water-level } \\
\text { altitude }\end{array}$ & Date & $\begin{array}{c}\text { Time } \\
\text { (24 hour) }\end{array}$ & $\begin{array}{l}\text { Water-level } \\
\text { altitude }\end{array}$ & Date & $\begin{array}{c}\text { Time } \\
\text { (24 hour) }\end{array}$ & $\begin{array}{l}\text { Water-level } \\
\text { altitude }\end{array}$ \\
\hline \multirow[t]{9}{*}{ 9/8/95 } & 1500 & $1,396.14$ & 9/11/95 & 0000 & $1,395.23$ & 9/13/95 & 0900 & $1,394.50$ \\
\hline & 1600 & $1,396.16$ & & 0100 & $1,395.22$ & & 1000 & $1,394.49$ \\
\hline & 1700 & $1,396.14$ & & 0200 & $1,395.20$ & & 1100 & $1,394.47$ \\
\hline & 1800 & $1,396.11$ & & 0300 & $1,395.18$ & & 1200 & $1,394.45$ \\
\hline & 1900 & $1,396.09$ & & 0400 & $1,395.16$ & & 1300 & $1,394.43$ \\
\hline & 2000 & $1,396.09$ & & 0500 & $1,395.15$ & & 1400 & $1,394.43$ \\
\hline & 2100 & $1,396.07$ & & 0600 & $1,395.13$ & & 1500 & $1,394.41$ \\
\hline & 2200 & $1,396.05$ & & 0700 & $1,395.08$ & & 1600 & $1,394.38$ \\
\hline & 2300 & $1,396.04$ & & 0800 & $1,395.09$ & & 1700 & $1,394.37$ \\
\hline \multirow[t]{24}{*}{ 9/9/95 } & 0000 & $1,396.02$ & & 0900 & $1,395.08$ & & 1800 & $1,394.34$ \\
\hline & 0100 & $1,396.01$ & & 1000 & $1,395.09$ & & 1900 & $1,394.33$ \\
\hline & 0200 & $1,396.00$ & & 1100 & $1,395.08$ & & 2000 & $1,394.31$ \\
\hline & 0300 & $1,395.98$ & & 1200 & $1,395.09$ & & 2100 & $1,394.28$ \\
\hline & 0400 & $1,395.97$ & & 1300 & $1,395.09$ & & 2200 & $1,394.25$ \\
\hline & 0500 & $1,395.95$ & & 1400 & $1,395.10$ & & 2300 & $1,394.23$ \\
\hline & 0600 & $1,395.94$ & & 1500 & $1,395.09$ & $9 / 14 / 95$ & 0000 & $1,394.21$ \\
\hline & 0700 & $1,395.89$ & & 1600 & $1,395.09$ & & 0100 & $1,394.19$ \\
\hline & 0800 & $1,395.89$ & & 1700 & $1,395.10$ & & 0200 & $1,394.17$ \\
\hline & 0900 & $1,395.88$ & & 1800 & $1,395.07$ & & 0300 & $1,394.15$ \\
\hline & 1000 & $1,395.86$ & & 1900 & $1,395.08$ & & 0400 & $1,394.13$ \\
\hline & 1100 & $1,395.86$ & & 2000 & $1,395.07$ & & 0500 & $1,394.11$ \\
\hline & 1200 & $1,395.84$ & & 2100 & $1,395.07$ & & 0600 & $1,394.08$ \\
\hline & 1300 & $1,395.83$ & & 2200 & $1,395.07$ & & 0700 & $1,394.04$ \\
\hline & 1400 & $1,395.81$ & & 2300 & $1,395.06$ & & 0800 & $1,394.01$ \\
\hline & 1500 & $1,395.82$ & $9 / 12 / 95$ & 0000 & $1,395.06$ & & 0900 & $1,394.01$ \\
\hline & 1600 & $1,395.78$ & & 0100 & $1,395.06$ & & 1000 & $1,393.99$ \\
\hline & 1700 & $1,395.78$ & & 0200 & $1,395.05$ & & 1100 & $1,393.97$ \\
\hline & 1800 & $1,395.75$ & & 0300 & $1,395.05$ & & 1200 & $1,393.97$ \\
\hline & 1900 & $1,395.74$ & & 0400 & $1,395.05$ & & 1300 & $1,393.95$ \\
\hline & 2000 & $1,395.72$ & & 0500 & $1,395.04$ & & 1400 & $1,393.97$ \\
\hline & 2100 & $1,395.70$ & & 0600 & $1,395.04$ & & 1500 & $1,393.88$ \\
\hline & 2200 & $1,395.68$ & & 0700 & $1,395.02$ & & 1600 & $1,393.88$ \\
\hline & 2300 & $1,395.66$ & & 0800 & $1,395.01$ & & 1700 & $1,393.87$ \\
\hline \multirow[t]{24}{*}{ 9/10/95 } & 0000 & $1,395.65$ & & 0900 & $1,395.02$ & & 1800 & $1,393.84$ \\
\hline & 0100 & $1,395.63$ & & 1000 & $1,395.00$ & & 1900 & $1,393.82$ \\
\hline & 0200 & $1,395.61$ & & 1100 & $1,394.99$ & & 2000 & $1,393.80$ \\
\hline & 0300 & $1,395.60$ & & 1200 & $1,394.98$ & & 2100 & $1,393.77$ \\
\hline & 0400 & $1,395.57$ & & 1300 & $1,394.96$ & & 2200 & $1,393.76$ \\
\hline & 0500 & $1,395.56$ & & 1400 & $1,394.94$ & & 2300 & $1,393.73$ \\
\hline & 0600 & $1,395.54$ & & 1500 & $1,394.92$ & 9/15/95 & 0000 & $1,393.70$ \\
\hline & 0700 & $1,395.50$ & & 1600 & $1,394.88$ & & 0100 & $1,393.69$ \\
\hline & 0800 & $1,395.49$ & & 1700 & $1,394.88$ & & 0200 & $1,393.67$ \\
\hline & 0900 & $1,395.48$ & & 1800 & $1,394.85$ & & 0300 & $1,393.64$ \\
\hline & 1000 & $1,395.47$ & & 1900 & $1,394.83$ & & 0400 & $1,393.63$ \\
\hline & 1100 & $1,395.47$ & & 2000 & $1,394.81$ & & 0500 & $1,393.60$ \\
\hline & 1200 & $1,395.43$ & & 2100 & $1,394.78$ & & 0600 & $1,393.59$ \\
\hline & 1300 & $1,395.40$ & & 2200 & $1,394.76$ & & 0700 & $1,393.54$ \\
\hline & 1400 & $1,395.43$ & & 2300 & $1,394.73$ & & 0800 & $1,393.53$ \\
\hline & 1500 & $1,395.40$ & $9 / 13 / 95$ & 0000 & $1,394.72$ & & 0900 & $1,393.50$ \\
\hline & 1600 & $1,395.38$ & 256 & 0100 & $1,394.70$ & & 1000 & $1,393.48$ \\
\hline & 1700 & $1,395.36$ & & 0200 & $1,394.68$ & & 1100 & $1,393.47$ \\
\hline & 1800 & $1,395.34$ & & 0300 & $1,394.65$ & & 1200 & $1,393.46$ \\
\hline & 1900 & $1,395.33$ & & 0400 & $1,394.63$ & & 1300 & $1,393.43$ \\
\hline & 2000 & $1,395.30$ & & 0500 & $1,394.61$ & & 1400 & $1,393.40$ \\
\hline & 2100 & $1,395.28$ & & 0600 & $1,394.59$ & & 1500 & $1,393.42$ \\
\hline & 2200 & $1,395.27$ & & 0700 & $1,394.55$ & & 1600 & $1,393.40$ \\
\hline & 2300 & $1,395.24$ & & 0800 & $1,394.52$ & & 1700 & $1,393.36$ \\
\hline
\end{tabular}


Table 8. Water-level altitudes for Trumble Road pond, July-September, 1995--Continued

\begin{tabular}{|c|c|c|c|c|c|c|c|c|}
\hline Date & $\begin{array}{c}\text { Time } \\
\text { (24 hour) }\end{array}$ & $\begin{array}{l}\text { Water-level } \\
\text { altitude }\end{array}$ & Date & $\begin{array}{c}\text { Time } \\
\text { (24 hour) }\end{array}$ & $\begin{array}{c}\text { Water-level } \\
\text { altitude }\end{array}$ & Date & $\begin{array}{c}\text { Time } \\
\text { (24 hour) }\end{array}$ & $\begin{array}{c}\text { Water-level } \\
\text { altitude }\end{array}$ \\
\hline \multirow[t]{6}{*}{$9 / 15 / 95$} & 1800 & $1,393.35$ & $9 / 18 / 95$ & 0300 & $1,392.10$ & 9/20/95 & 1200 & $1,390.69$ \\
\hline & 1900 & $1,393.32$ & & 0400 & $1,392.08$ & & 1300 & $1,390.67$ \\
\hline & 2000 & $1,393.30$ & & 0500 & $1,392.06$ & & 1400 & $1,390.64$ \\
\hline & 2100 & $1,393.27$ & & 0600 & $1,392.03$ & & 1500 & $1,390.62$ \\
\hline & 2200 & $1,393.25$ & & 0700 & $1,392.00$ & & 1600 & $1,390.60$ \\
\hline & 2300 & $1,393.23$ & & 0800 & $1,391.96$ & & 1700 & $1,390.58$ \\
\hline \multirow[t]{24}{*}{ 9/16/95 } & 0000 & $1,393.21$ & & 0900 & $1,391.94$ & & 1800 & $1,390.55$ \\
\hline & 0100 & $1,393.19$ & & 1000 & $1,391.94$ & & 1900 & $1,390.53$ \\
\hline & 0200 & $1,393.17$ & & 1100 & $1,391.91$ & & 2000 & $1,390.50$ \\
\hline & 0300 & $1,393.14$ & & 1200 & $1,391.89$ & & 2100 & $1,390.47$ \\
\hline & 0400 & $1,393.12$ & & 1300 & $1,391.91$ & & 2200 & $1,390.45$ \\
\hline & 0500 & $1,393.10$ & & 1400 & $1,391.87$ & & 2300 & $1,390.43$ \\
\hline & 0600 & $1,393.09$ & & 1500 & $1,391.85$ & 9/21/95 & 0000 & $1,390.41$ \\
\hline & 0700 & $1,393.06$ & & 1600 & $1,391.82$ & & 0100 & $1,390.39$ \\
\hline & 0800 & $1,393.04$ & & 1700 & $1,391.80$ & & 0200 & $1,390.37$ \\
\hline & 0900 & $1,392.98$ & & 1800 & $1,391.76$ & & 0300 & $1,390.34$ \\
\hline & 1000 & $1,392.99$ & & 1900 & $1,391.74$ & & 0400 & $1,390.32$ \\
\hline & 1100 & $1,392.98$ & & 2000 & $1,391.71$ & & 0500 & $1,390.30$ \\
\hline & 1200 & $1,392.94$ & & 2100 & $1,391.68$ & & 0600 & $1,390.28$ \\
\hline & 1300 & $1,392.94$ & & 2200 & $1,391.65$ & & 0700 & $1,390.24$ \\
\hline & 1400 & $1,392.91$ & & 2300 & $1,391.63$ & & 0800 & $1,390.20$ \\
\hline & 1500 & $1,392.91$ & $9 / 19 / 95$ & 0000 & $1,391.60$ & & 0900 & $1,390.18$ \\
\hline & 1600 & $1,392.89$ & & 0100 & $1,391.58$ & & 1000 & $1,390.17$ \\
\hline & 1700 & $1,392.87$ & & 0200 & $1,391.55$ & & 1100 & $1,390.16$ \\
\hline & 1800 & $1,392.83$ & & 0300 & $1,391.53$ & & 1200 & $1,390.17$ \\
\hline & 1900 & $1,392.82$ & & 0400 & $1,391.50$ & & 1300 & $1,390.14$ \\
\hline & 2000 & $1,392.80$ & & 0500 & $1,391.48$ & & 1400 & $1,390.10$ \\
\hline & 2100 & $1,392.77$ & & 0600 & $1,391.45$ & & 1500 & $1,390.09$ \\
\hline & 2200 & $1,392.75$ & & 0700 & $1,391.41$ & & 1600 & $1,390.08$ \\
\hline & 2300 & $1,392.72$ & & 0800 & $1,391.37$ & & 1700 & $1,390.04$ \\
\hline \multirow[t]{24}{*}{ 9/17/95 } & 0000 & $1,392.70$ & & 0900 & $1,391.35$ & & 1800 & $1,390.03$ \\
\hline & 0100 & $1,392.68$ & & 1000 & $1,391.34$ & & 1900 & $1,390.00$ \\
\hline & 0200 & $1,392.66$ & & 1100 & $1,391.31$ & & 2000 & $1,389.98$ \\
\hline & 0300 & $1,392.63$ & & 1200 & $1,391.29$ & & 2100 & $1,389.96$ \\
\hline & 0400 & $1,392.61$ & & 1300 & $1,391.28$ & & 2200 & $1,389.93$ \\
\hline & 0500 & $1,392.59$ & & 1400 & $1,391.25$ & & 2300 & $1,389.91$ \\
\hline & 0600 & $1,392.57$ & & 1500 & $1,391.22$ & $9 / 22 / 95$ & 0000 & $1,389.89$ \\
\hline & 0700 & $1,392.54$ & & 1600 & $1,391.21$ & & 0100 & $1,389.86$ \\
\hline & 0800 & $1,392.51$ & & 1700 & $1,391.18$ & & 0200 & $1,389.84$ \\
\hline & 0900 & $1,392.48$ & & 1800 & $1,391.15$ & & 0300 & $1,389.82$ \\
\hline & 1000 & $1,392.47$ & & 1900 & $1,391.12$ & & 0400 & $1,389.80$ \\
\hline & 1100 & $1,392.44$ & & 2000 & $1,391.09$ & & 0500 & $1,389.78$ \\
\hline & 1200 & $1,392.45$ & & 2100 & $1,391.07$ & & 0600 & $1,389.76$ \\
\hline & 1300 & $1,392.42$ & & 2200 & $1,391.04$ & & 0700 & $1,389.72$ \\
\hline & 1400 & $1,392.40$ & & 2300 & $1,391.02$ & & 0800 & $1,389.68$ \\
\hline & 1500 & $1,392.37$ & $9 / 20 / 95$ & 0000 & $1,390.99$ & & 0900 & $1,389.66$ \\
\hline & 1600 & $1,392.36$ & & 0100 & $1,390.97$ & & 1000 & $1,389.64$ \\
\hline & 1700 & $1,392.33$ & & 0200 & $1,390.94$ & & 1100 & $1,389.64$ \\
\hline & 1800 & $1,392.31$ & & 0300 & $1,390.92$ & & 1200 & $1,389.60$ \\
\hline & 1900 & $1,392.29$ & & 0400 & $1,390.89$ & & 1300 & $1,389.58$ \\
\hline & 2000 & $1,392.26$ & & 0500 & $1,390.86$ & & 1400 & $1,389.57$ \\
\hline & 2100 & $1,392.24$ & & 0600 & $1,390.84$ & & 1500 & $1,389.54$ \\
\hline & 2200 & $1,392.21$ & & 0700 & $1,390.81$ & & 1600 & $1,389.52$ \\
\hline & 2300 & $1,392.19$ & & 0800 & $1,390.77$ & & 1700 & $1,389.49$ \\
\hline \multirow[t]{3}{*}{ 9/18/95 } & 0000 & $1,392.17$ & & 0900 & $1,390.73$ & & 1800 & $1,389.45$ \\
\hline & 0100 & $1,392.15$ & & 1000 & $1,390.72$ & & 1900 & $1,389.43$ \\
\hline & 0200 & $1,392.12$ & & 1100 & $1,390.71$ & & 2000 & $1,389.39$ \\
\hline
\end{tabular}


Tab/e 8. Water-level altitudes for Trumble Road pond, July-September, 1995--Continued

\begin{tabular}{|c|c|c|c|c|c|}
\hline Date & $\begin{array}{c}\text { Time } \\
\text { (24 hour) }\end{array}$ & $\begin{array}{c}\text { Water-level } \\
\text { altitude }\end{array}$ & Date & $\begin{array}{c}\text { Time } \\
\text { (24 hour) }\end{array}$ & $\begin{array}{c}\text { Water-level } \\
\text { altitude }\end{array}$ \\
\hline \multirow[t]{3}{*}{$9 / 22 / 95$} & 2100 & $1,389.36$ & $9 / 25 / 95$ & 0400 & $1,387.91$ \\
\hline & 2200 & $1,389.33$ & & 0500 & $1,387.88$ \\
\hline & 2300 & $1,389.30$ & & 0600 & $1,387.85$ \\
\hline \multirow[t]{24}{*}{$9 / 23 / 95$} & 0000 & $1,389.27$ & & 0700 & $1,387.82$ \\
\hline & 0100 & $1,389.25$ & & 0800 & $1,387.79$ \\
\hline & 0200 & $1,389.21$ & & 0900 & $1,387.78$ \\
\hline & 0300 & $1,389.19$ & & 1000 & $1,387.74$ \\
\hline & 0400 & $1,389.16$ & & 1100 & $1,387.77$ \\
\hline & 0500 & $1,389.13$ & & 1200 & $1,387.70$ \\
\hline & 0600 & $1,389.11$ & & 1300 & $1,387.77$ \\
\hline & 0700 & $1,389.07$ & & 1400 & $1,387.69$ \\
\hline & 0800 & $1,389.02$ & & 1500 & $1,387.66$ \\
\hline & 0900 & $1,389.00$ & & 1600 & $1,387.63$ \\
\hline & 1000 & $1,388.98$ & & 1700 & $1,387.60$ \\
\hline & 1100 & $1,388.95$ & & 1800 & $1,387.57$ \\
\hline & 1200 & $1,388.99$ & & 1900 & $1,387.54$ \\
\hline & 1300 & $1,388.94$ & & 2000 & $1,387.51$ \\
\hline & 1400 & $1,388.91$ & & 2100 & $1,387.48$ \\
\hline & 1500 & $1,388.89$ & $9 / 26 / 95$ & 2200 & $1,387.45$ \\
\hline & 1600 & $1,388.84$ & & 2300 & $1,387.42$ \\
\hline & 1700 & $1,388.81$ & & 0000 & $1,387.40$ \\
\hline & 1800 & $1,388.78$ & & 0100 & $1,387.37$ \\
\hline & 1900 & $1,388.75$ & & 0200 & $1,387.34$ \\
\hline & 2000 & $1,388.71$ & & 0300 & $1,387.31$ \\
\hline & 2100 & $1,388.68$ & & 0400 & $1,387.28$ \\
\hline & 2200 & $1,388.65$ & & 0500 & $1,387.25$ \\
\hline & 2300 & $1,388.62$ & & 0600 & $1,387.21$ \\
\hline \multirow[t]{24}{*}{$9 / 24 / 95$} & 0000 & $1,388.60$ & & 0700 & $1,387.19$ \\
\hline & 0100 & $1,388.56$ & & 0800 & $1,387.15$ \\
\hline & 0200 & $1,388.54$ & & 0900 & $1,387.11$ \\
\hline & 0300 & $1,388.51$ & & 1000 & $1,387.05$ \\
\hline & 0400 & $1,388.48$ & & 1100 & $1,387.05$ \\
\hline & 0500 & $1,388.45$ & & 1200 & $1,387.03$ \\
\hline & 0600 & $1,388.42$ & & 1300 & $1,386.99$ \\
\hline & 0700 & $1,388.38$ & & 1400 & $1,386.95$ \\
\hline & 0800 & $1,388.35$ & & 1500 & $1,386.92$ \\
\hline & 0900 & $1,388.32$ & & 1600 & $1,386.88$ \\
\hline & 1000 & $1,388.32$ & & 1700 & $1,386.84$ \\
\hline & 1100 & $1,388.31$ & & 1800 & $1,386.81$ \\
\hline & 1200 & $1,388.29$ & & 1900 & $1,386.77$ \\
\hline & 1300 & $1,388.23$ & & 2000 & $1,386.73$ \\
\hline & 1400 & $1,388.25$ & & 2100 & $1,386.69$ \\
\hline & 1500 & $1,388.24$ & $9 / 27 / 95$ & 2200 & $1,386.65$ \\
\hline & 1600 & $1,388.20$ & & 2300 & $1,386.61$ \\
\hline & 1700 & $1,388.18$ & & 0000 & $1,386.57$ \\
\hline & 1800 & $1,388.15$ & & 0100 & $1,386.53$ \\
\hline & 1900 & $1,388.13$ & & 0200 & $1,386.49$ \\
\hline & 2000 & $1,388.10$ & & 0300 & $1,386.45$ \\
\hline & 2100 & $1,388.07$ & & 0400 & $1,386.41$ \\
\hline & 2200 & $1,388.05$ & & 0500 & $1,386.37$ \\
\hline & 2300 & $1,388.02$ & & 0600 & $1,386.33$ \\
\hline \multirow[t]{4}{*}{$9 / 25 / 95$} & 0000 & $1,388.00$ & & 0700 & $1,386.29$ \\
\hline & 0100 & $1,387.98$ & & 0800 & $1,386.24$ \\
\hline & 0200 & $1,387.96$ & & 0900 & $1,386.20$ \\
\hline & 0300 & $1,387.93$ & & & \\
\hline
\end{tabular}


Table 9. Water-level altitudes for Winchester pond B (west pond), June-September, 1995

[Water-level altitude in feet above sea level, rounded to the nearest hundredth]

\begin{tabular}{|c|c|c|c|c|c|c|c|c|}
\hline Date & $\begin{array}{c}\text { Time } \\
\text { (24 hour) }\end{array}$ & $\begin{array}{c}\text { Water-level } \\
\text { altitude }\end{array}$ & Date & $\begin{array}{c}\text { Time } \\
\text { (24 hour) }\end{array}$ & $\begin{array}{c}\text { Water-level } \\
\text { altitude }\end{array}$ & Date & $\begin{array}{c}\text { Time } \\
\text { (24 hour) }\end{array}$ & $\begin{array}{l}\text { Water-level } \\
\text { altitude }\end{array}$ \\
\hline \multirow[t]{9}{*}{$6 / 21 / 95$} & 1515 & $1,448.41$ & $6 / 24 / 95$ & 0100 & $1,448.30$ & $6 / 26 / 95$ & 1300 & $1,449.61$ \\
\hline & 1600 & $1,448.40$ & & 0200 & $1,448.29$ & & 1400 & $1,449.61$ \\
\hline & 1700 & $1,448.39$ & & 0300 & $1,448.28$ & & 1500 & $1,449.62$ \\
\hline & 1800 & $1,448.40$ & & 0400 & $1,448.26$ & & 1600 & $1,449.62$ \\
\hline & 1900 & $1,448.37$ & & 0500 & $1,448.25$ & & 1700 & $1,449.61$ \\
\hline & 2000 & $1,448.37$ & & 0600 & $1,448.24$ & & 1800 & $1,449.63$ \\
\hline & 2100 & $1,448.36$ & & 0700 & $1,448.23$ & & 1900 & $1,449.61$ \\
\hline & 2200 & $1,448.35$ & & 0800 & $1,448.23$ & & 2000 & $1,449.59$ \\
\hline & 2300 & $1,448.33$ & & 0900 & $1,448.27$ & & 2100 & $1,449.61$ \\
\hline \multirow[t]{24}{*}{$6 / 22 / 95$} & 0000 & $1,448.33$ & & 1000 & $1,448.30$ & & 2200 & $1,449.60$ \\
\hline & 0100 & $1,448.32$ & & 1100 & $1,448.33$ & & 2300 & $1,449.60$ \\
\hline & 0200 & $1,448.32$ & & 1200 & $1,448.36$ & $6 / 27 / 95$ & 0000 & $1,449.60$ \\
\hline & 0300 & $1,448.32$ & & 1300 & $1,448.40$ & & 0100 & $1,449.60$ \\
\hline & 0400 & $1,448.32$ & & 1400 & $1,448.44$ & & 0200 & $1,449.60$ \\
\hline & 0500 & $1,448.32$ & & 1500 & $1,448.48$ & & 0300 & $1,449.60$ \\
\hline & 0600 & $1,448.32$ & & 1600 & $1,448.52$ & & 0400 & $1,449.61$ \\
\hline & 0700 & $1,448.31$ & & 1700 & $1,448.56$ & & 0500 & $1,449.61$ \\
\hline & 0800 & $1,448.32$ & & 1800 & $1,448.59$ & & 0600 & $1,449.61$ \\
\hline & 0900 & $1,448.32$ & & 1900 & $1,448.64$ & & 0700 & $1,449.61$ \\
\hline & 1000 & $1,448.33$ & & 2000 & $1,448.68$ & & 0800 & $1,449.61$ \\
\hline & 1100 & $1,448.33$ & & 2100 & $1,448.72$ & & 0900 & $1,449.61$ \\
\hline & 1200 & $1,448.34$ & & 2200 & $1,448.74$ & & 1000 & $1,449.61$ \\
\hline & 1300 & $1,448.34$ & & 2300 & $1,448.78$ & & 1100 & $1,449.61$ \\
\hline & 1400 & $1,448.36$ & $6 / 25 / 95$ & 0000 & $1,448.80$ & & 1200 & $1,449.61$ \\
\hline & 1500 & $1,448,35$ & & 0100 & $1,448.83$ & & 1300 & $1,449.61$ \\
\hline & 1600 & $1,448.37$ & & 0200 & $1,448.86$ & & 1400 & $1,449.61$ \\
\hline & 1700 & $1,448.37$ & & 0300 & $1,448.90$ & & 1500 & $1,449.60$ \\
\hline & 1800 & $1,448.38$ & & 0400 & $1,448.93$ & & 1600 & $1,449.60$ \\
\hline & 1900 & $1,448.37$ & & 0500 & $1,448.96$ & & 1700 & $1,449.58$ \\
\hline & 2000 & $1,448.38$ & & 0600 & $1,448.98$ & & 1800 & $1,449.58$ \\
\hline & 2100 & $1,448.38$ & & 0700 & $1,449.01$ & & 1900 & $1,449.59$ \\
\hline & 2200 & $1,448.37$ & & 0800 & $1,449.03$ & & 2000 & $1,449.58$ \\
\hline & 2300 & $1,448.36$ & & 0900 & $1,449.06$ & & 2100 & $1,449.58$ \\
\hline \multirow[t]{24}{*}{ 6/23/95 } & 0000 & $1,448.35$ & & 1000 & $1,449.09$ & & 2200 & $1,449.57$ \\
\hline & 0100 & $1,448.33$ & & 1100 & $1,449.12$ & & 2300 & $1,449.56$ \\
\hline & 0200 & $1,448.33$ & & 1200 & $1,449.15$ & $6 / 28 / 95$ & 0000 & $1,449.55$ \\
\hline & 0300 & $1,448.32$ & & 1300 & $1,449.18$ & & 0100 & $1,449.55$ \\
\hline & 0400 & $1,448.31$ & & 1400 & $1,449.22$ & & 0200 & $1,449.55$ \\
\hline & 0500 & $1,448.30$ & & 1500 & $1,449.25$ & & 0300 & $1,449.54$ \\
\hline & 0600 & $1,448.29$ & & 1600 & $1,449.29$ & & 0400 & $1,449.54$ \\
\hline & 0700 & $1,448.28$ & & 1700 & $1,449.31$ & & 0500 & $1,449.54$ \\
\hline & 0800 & $1,448.28$ & & 1800 & $1,449.34$ & & 0600 & $1,449.54$ \\
\hline & 0900 & $1,448.29$ & & 1900 & $1,449.36$ & & 0700 & $1,449.54$ \\
\hline & 1000 & $1,448.29$ & & 2000 & $1,449.40$ & & 0800 & $1,449.54$ \\
\hline & 1100 & $1,448.30$ & & 2100 & $1,449.43$ & & 0900 & $1,449.54$ \\
\hline & 1200 & $1,448.30$ & & 2200 & $1,449.45$ & & 1000 & $1,449.55$ \\
\hline & 1300 & $1,448.31$ & & 2300 & $1,449.47$ & & 1100 & $1,449.55$ \\
\hline & 1400 & $1,448.31$ & $6 / 26 / 95$ & 0000 & $1,449.49$ & & 1200 & $1,449.54$ \\
\hline & 1500 & $1,448.30$ & & 0100 & $1,449.51$ & & 1300 & $1,449.53$ \\
\hline & 1600 & $1,448.32$ & & 0200 & $1,449.53$ & & 1400 & $1,449.52$ \\
\hline & 1700 & $1,448.31$ & & 0300 & $1,449.55$ & & 1500 & $1,449.53$ \\
\hline & 1800 & $1,448.31$ & & 0400 & $1,449.57$ & & 1600 & $1,449.51$ \\
\hline & 1900 & $1,448.32$ & & 0500 & $1,449.60$ & & 1700 & $1,449.50$ \\
\hline & 2000 & $1,448.31$ & & 0600 & $1,449.62$ & & 1800 & $1,449.49$ \\
\hline & 2100 & $1,448.32$ & & 0700 & $1,449.64$ & & 1900 & $1,449.49$ \\
\hline & 2200 & $1,448.32$ & & 0800 & $1,449.65$ & & 2000 & $1,449.49$ \\
\hline & 2300 & $1,448.32$ & & 1100 & $1,449.63$ & & 2100 & $1,449.49$ \\
\hline $6 / 24 / 95$ & 0000 & $1,448.31$ & & 1200 & $1,449.62$ & & 2200 & $1,449.48$ \\
\hline
\end{tabular}


Table 9. Water-level altitudes for Winchester pond B (west pond), June-September, 1995--Continued

\begin{tabular}{|c|c|c|c|c|c|c|c|c|}
\hline Date & $\begin{array}{c}\text { Time } \\
\text { (24 hour) }\end{array}$ & $\begin{array}{l}\text { Water-level } \\
\text { altitude }\end{array}$ & Date & $\begin{array}{c}\text { Time } \\
\text { (24 hour) }\end{array}$ & $\begin{array}{l}\text { Water-level } \\
\text { altitude }\end{array}$ & Date & $\begin{array}{c}\text { Time } \\
\text { (24 hour) }\end{array}$ & $\begin{array}{l}\text { Water-level } \\
\text { altitude }\end{array}$ \\
\hline $6 / 28 / 95$ & 2300 & $1,449.47$ & $7 / 15 / 95$ & 0600 & $1,449.68$ & $7 / 17 / 95$ & 1600 & $1,449.47$ \\
\hline \multirow[t]{12}{*}{ 6/29/95 } & 0000 & $1,449.46$ & & 0700 & $1,449.66$ & & 1700 & $1,449.44$ \\
\hline & 0100 & $1,449.46$ & & 0800 & $1,449.65$ & & 1800 & $1,449.44$ \\
\hline & 0200 & $1,449.45$ & & 0900 & $1,449.65$ & & 1900 & $1,449.41$ \\
\hline & 0300 & $1,449.44$ & & 1000 & $1,449.65$ & & 2000 & $1,449.40$ \\
\hline & 0400 & $1,449.44$ & & 1100 & $1,449.65$ & & 2100 & $1,449.40$ \\
\hline & 0500 & $1,449.43$ & & 1200 & $1,449.65$ & & 2200 & $1,449.38$ \\
\hline & 0600 & $1,449.42$ & & 1300 & $1,449.65$ & & 2300 & $1,449.38$ \\
\hline & 0700 & $1,449.41$ & & 1400 & $1,449.66$ & $7 / 18 / 95$ & 0000 & $1,449.36$ \\
\hline & 0800 & $1,449.41$ & & 1500 & $1,449.65$ & & 0100 & $1,449.35$ \\
\hline & 0900 & $1,449.41$ & & 1600 & $1,449.66$ & & 0200 & $1,449.35$ \\
\hline & 1000 & $1,449.40$ & & 1700 & $1,449.64$ & & 0300 & $1,449.34$ \\
\hline & 1100 & $1,449.40$ & & 1800 & $1,449.66$ & & 0400 & $1,449.34$ \\
\hline \multirow[t]{15}{*}{$7 / 13 / 95$} & 0900 & $1,450.00$ & & 1900 & $1,449.65$ & & 0500 & $1,449.33$ \\
\hline & 1000 & $1,450.02$ & & 2000 & $1,449.65$ & & 0600 & $1,449.32$ \\
\hline & 1100 & $1,450.04$ & & 2100 & $1,449.66$ & & 0700 & $1,449.31$ \\
\hline & 1200 & $1,450.04$ & & 2200 & $1,449.66$ & & 0800 & $1,449.30$ \\
\hline & 1300 & $1,450.05$ & & 2300 & $1,449.65$ & & 0900 & $1,449.29$ \\
\hline & 1400 & $1,450.06$ & $7 / 16 / 95$ & 0000 & $1,449.66$ & & 1000 & $1,449.30$ \\
\hline & 1500 & $1,450.03$ & & 0100 & $1,449.65$ & & 1100 & $1,449.30$ \\
\hline & 1600 & $1,450.00$ & & 0200 & $1,449.65$ & & 1200 & $1,449.28$ \\
\hline & 1700 & $1,449.97$ & & 0300 & $1,449.65$ & & 1300 & $1,449.27$ \\
\hline & 1800 & $1,449.97$ & & 0400 & $1,449.65$ & & 1400 & $1,449.25$ \\
\hline & 1900 & $1,449.97$ & & 0500 & $1,449.65$ & & 1500 & $1,449.23$ \\
\hline & 2000 & $1,449.96$ & & 0600 & $1,449.65$ & & 1600 & $1,449.22$ \\
\hline & 2100 & $1,449.95$ & & 0700 & $1,449.64$ & & 1700 & $1,449.19$ \\
\hline & 2200 & $1,449.94$ & & 0800 & $1,449.65$ & & 1800 & $1,449.19$ \\
\hline & 2300 & $1,449.95$ & & 0900 & $1,449.65$ & & 1900 & $1,449.17$ \\
\hline \multirow[t]{24}{*}{$7 / 14 / 95$} & 0000 & $1,449.95$ & & 1000 & $1,449.64$ & & 2000 & 1.449 .16 \\
\hline & 0100 & $1,449.93$ & & 1100 & $1,449.66$ & & 2100 & $1,449.14$ \\
\hline & 0200 & $1,449.93$ & & 1200 & $1,449.64$ & & 2200 & $1,449.14$ \\
\hline & 0300 & $1,449.94$ & & 1300 & $1,449.65$ & & 2300 & $1,449.16$ \\
\hline & 0400 & $1,449.94$ & & 1400 & $1,449.65$ & $7 / 19 / 95$ & 0000 & $1,449.15$ \\
\hline & 0500 & $1,449.93$ & & 1500 & $1,449.64$ & & 0100 & $1,449.14$ \\
\hline & 0600 & $1,449.93$ & & 1600 & $1,449.64$ & & 0200 & $1,449.14$ \\
\hline & 0700 & $1,449.93$ & & 1700 & $1,449.64$ & & 0300 & $1,449.15$ \\
\hline & 0800 & $1,449.93$ & & 1800 & $1,449.65$ & & 0400 & $1,449.15$ \\
\hline & 0900 & $1,449.92$ & & 1900 & $1,449.65$ & & 0500 & $1,449.14$ \\
\hline & 1000 & $1,449.93$ & & 2000 & $1,449.64$ & & 0600 & $1,449.14$ \\
\hline & 1100 & $1,449.93$ & & 2100 & $1,449.64$ & & 0700 & $1,449.14$ \\
\hline & 1200 & $1,449.91$ & & 2200 & $1,449.63$ & & 0800 & $1,449.14$ \\
\hline & 1300 & $1,449.90$ & & 2300 & $1,449.64$ & & 0900 & $1,449.14$ \\
\hline & 1400 & $1,449.87$ & $7 / 17 / 95$ & 0000 & $1,449.63$ & & 1000 & $1,449.14$ \\
\hline & 1500 & $1,449.87$ & & 0100 & $1,449.63$ & & 1100 & $1,449.15$ \\
\hline & 1600 & $1,449.86$ & & 0200 & $1,449.63$ & & 1200 & $1,449.12$ \\
\hline & 1700 & $1,449.83$ & & 0300 & $1,449.63$ & & 1300 & $1,449.10$ \\
\hline & 1800 & $1,449.81$ & & 0400 & $1,449.63$ & & 1400 & $1,449.08$ \\
\hline & 1900 & $1,449.79$ & & 0500 & $1,449.62$ & & 1500 & $1,449.08$ \\
\hline & 2000 & $1,449.78$ & & 0600 & $1,449.62$ & & 1600 & $1,449.06$ \\
\hline & 2100 & $1,449.77$ & & 0700 & $1,449.61$ & & 1700 & $1,449.04$ \\
\hline & 2200 & $1,449.76$ & & 0800 & $1,449.60$ & & 1800 & $1,448.96$ \\
\hline & 2300 & $1,449.75$ & & 0900 & $1,449.59$ & & 1900 & $1,448.99$ \\
\hline \multirow[t]{6}{*}{$7 / 15 / 95$} & 0000 & $1,449.72$ & & 1000 & $1,449.59$ & & 2000 & $1,448.99$ \\
\hline & 0100 & $1,449.72$ & & 1100 & $1,449.56$ & & 2100 & $1,449.00$ \\
\hline & 0200 & $1,449.72$ & & 1200 & $1,449.55$ & & 2200 & $1,448.99$ \\
\hline & 0300 & $1,449.70$ & & 1300 & $1,449.53$ & & 2300 & $1,448.98$ \\
\hline & 0400 & $1,449.69$ & & 1400 & $1,449.50$ & $7 / 20 / 95$ & 0000 & $1,448.99$ \\
\hline & 0500 & $1,449.68$ & & 1500 & $1,449.47$ & & 0100 & $1,448.99$ \\
\hline
\end{tabular}


Table 9. Water-level altitudes for Winchester pond B (west pond), June-September, 1995--Continued

\begin{tabular}{|c|c|c|c|c|c|c|c|c|}
\hline Date & $\begin{array}{c}\text { Time } \\
\text { (24 hour) }\end{array}$ & $\begin{array}{c}\text { Water-level } \\
\text { altitude }\end{array}$ & Date & $\begin{array}{c}\text { Time } \\
\text { (24 hour) }\end{array}$ & $\begin{array}{c}\text { Water-level } \\
\text { altitude }\end{array}$ & Date & $\begin{array}{c}\text { Time } \\
\text { (24 hour) }\end{array}$ & $\begin{array}{l}\text { Water-level } \\
\text { altitude }\end{array}$ \\
\hline \multirow[t]{22}{*}{ 7/20/95 } & 0200 & $1,448.99$ & $7 / 22 / 95$ & 1200 & $1,448.53$ & $7 / 24 / 95$ & 2200 & $1,448.32$ \\
\hline & 0300 & $1,448.98$ & & 1300 & $1,448.53$ & & 2300 & $1,448.33$ \\
\hline & 0400 & $1,448.99$ & & 1400 & $1,448.52$ & $7 / 25 / 95$ & 0000 & $1,448.33$ \\
\hline & 0500 & $1,448.98$ & & 1500 & $1,448.53$ & & 0100 & $1,448.32$ \\
\hline & 0600 & $1,448.98$ & & 1600 & $1,448.55$ & & 0200 & $1,448.32$ \\
\hline & 0700 & $1,448.97$ & & 1700 & $1,448.55$ & & 0300 & $1,448.32$ \\
\hline & 0800 & $1,448.96$ & & 1800 & $1,448.54$ & & 0400 & $1,448.32$ \\
\hline & 0900 & $1,448.95$ & & 1900 & $1,448.51$ & & 0500 & $1,448.31$ \\
\hline & 1000 & 1.448 .95 & & 2000 & $1,448.50$ & & 0600 & $1,448.31$ \\
\hline & 1100 & $1,448.95$ & & 2100 & $1,448.50$ & & 0700 & $1,448.30$ \\
\hline & 1200 & $1,448.94$ & & 2200 & $1,448.50$ & & 0800 & $1,448.30$ \\
\hline & 1300 & $1,448.93$ & & 2300 & $1,448.50$ & & 0900 & $1,448.30$ \\
\hline & 1400 & $1,448.86$ & $7 / 23 / 95$ & 0000 & $1,448.49$ & & 1000 & $1,448.29$ \\
\hline & 1500 & $1,448.87$ & & 0100 & $1,448.49$ & & 1100 & $1,448.28$ \\
\hline & 1600 & $1,448.84$ & & 0200 & $1,448.48$ & & 1200 & $1,448.26$ \\
\hline & 1700 & $1,448.86$ & & 0300 & $1,448.48$ & & 1300 & $1,448.25$ \\
\hline & 1800 & $1,448.81$ & & 0400 & $1,448.47$ & & 1400 & $1,448.23$ \\
\hline & 1900 & $1,448.82$ & & 0500 & $1,448.47$ & & 1500 & $1,448.21$ \\
\hline & 2000 & $1,448.79$ & & 0600 & $1,448.47$ & & 1600 & $1,448.20$ \\
\hline & 2100 & $1,448.78$ & & 0700 & $1,448.46$ & & 1700 & $1,448.21$ \\
\hline & 2200 & $1,448.78$ & & 0800 & $1,448.46$ & & 1800 & $1,448.17$ \\
\hline & 2300 & $1,448.77$ & & 0900 & $1,448.47$ & & 1900 & $1,448.17$ \\
\hline \multirow[t]{24}{*}{ 7/21/95 } & 0000 & $1,448.77$ & & 1000 & $1,448.46$ & & 2000 & $1,448.15$ \\
\hline & 0100 & $1,448.77$ & & 1100 & $1,448.46$ & & 2100 & $1,448.13$ \\
\hline & 0200 & $1,448.77$ & & 1200 & $1,448.46$ & & 2200 & $1,448.13$ \\
\hline & 0300 & $1,448.77$ & & 1300 & $1,448.47$ & & 2300 & $1,448.11$ \\
\hline & 0400 & $1,448.76$ & & 1400 & $1,448.47$ & $7 / 26 / 95$ & 0000 & $1,448.11$ \\
\hline & 0500 & $1,448.76$ & & 1500 & $1,448.48$ & & 0100 & $1,448.09$ \\
\hline & 0600 & $1,448.76$ & & 1600 & $1,448.46$ & & 0200 & $1,448.09$ \\
\hline & 0700 & $1,448.75$ & & 1700 & $1,448.47$ & & 0300 & $1,448.08$ \\
\hline & 0800 & $1,448.75$ & & 1800 & $1,448.47$ & & 0400 & $1,448.08$ \\
\hline & 0900 & $1,448.74$ & & 1900 & $1,448.49$ & & 0500 & $1,448.07$ \\
\hline & 1000 & $1,448.74$ & & 2000 & $1,448.48$ & & 0600 & $1,448.06$ \\
\hline & 1100 & $1,448.73$ & & 2100 & $1,448.48$ & & 0700 & $1,448.05$ \\
\hline & 1200 & $1,448.71$ & & 2200 & $1,448.48$ & & 0800 & $1,448.05$ \\
\hline & 1300 & $1,448.69$ & & 2300 & $1,448.48$ & & 0900 & $1,448.04$ \\
\hline & 1400 & $1,448.68$ & $7 / 24 / 95$ & 0000 & $1,448.48$ & & 1000 & $1,448.04$ \\
\hline & 1500 & $1,448.68$ & & 0100 & $1,448.48$ & & 1100 & $1,448.04$ \\
\hline & 1600 & $1,448.66$ & & 0200 & $1,448.47$ & & 1200 & $1,448.03$ \\
\hline & 1700 & $1,448.63$ & & 0300 & $1,448.48$ & & 1300 & $1,448.01$ \\
\hline & 1800 & $1,448.59$ & & 0400 & $1,448.47$ & & 1400 & $1,448.00$ \\
\hline & 1900 & $1,448.58$ & & 0500 & $1,448.47$ & & 1500 & $1,447.97$ \\
\hline & 2000 & $1,448.61$ & & 0600 & $1,448.47$ & & 1600 & $1,447.99$ \\
\hline & 2100 & $1,448.59$ & & 0700 & $1,448.46$ & & 1700 & $1,447.98$ \\
\hline & 2200 & $1,448.60$ & & 0800 & $1,448.45$ & & 1800 & $1,447.98$ \\
\hline & 2300 & $1,448.59$ & & 0900 & $1,448.44$ & & 1900 & $1,447.96$ \\
\hline \multirow[t]{12}{*}{$7 / 22 / 95$} & 0000 & $1,448.58$ & & 1000 & $1,448.44$ & & 2000 & $1,447.95$ \\
\hline & 0100 & $1,448.59$ & & 1100 & $1,448.43$ & & 2100 & $1,447.95$ \\
\hline & 0200 & $1,448.58$ & & 1200 & $1,448.42$ & & 2200 & $1,447.95$ \\
\hline & 0300 & $1,448.57$ & & 1300 & $1,448.41$ & & 2300 & $1,447.95$ \\
\hline & 0400 & $1,448.57$ & & 1400 & $1,448.40$ & $7 / 27 / 95$ & 0000 & $1,447.94$ \\
\hline & 0500 & $1,448.56$ & & 1500 & $1,448.38$ & & 0100 & $1,447.94$ \\
\hline & 0600 & $1,448.55$ & & 1600 & $1,448.35$ & & 0200 & $1,447.94$ \\
\hline & 0700 & $1,448.54$ & & 1700 & $1,448.37$ & & 0300 & $1,447.94$ \\
\hline & 0800 & $1,448.53$ & & 1800 & $1,448.34$ & & 0400 & $1,447.94$ \\
\hline & 0900 & $1,448.53$ & & 1900 & $1,448.32$ & & 0500 & $1,447.93$ \\
\hline & 1000 & $1,448.53$ & & 2000 & $1,448.35$ & & 0600 & $1,447.93$ \\
\hline & 1100 & $1,448.53$ & & 2100 & $1,448.33$ & & 0700 & $1,447.93$ \\
\hline
\end{tabular}


Table 9. Water-level altitudes for Winchester pond B (west pond), June-September, 1995--Continued

\begin{tabular}{|c|c|c|c|c|c|c|c|c|}
\hline Date & $\begin{array}{c}\text { Time } \\
\text { (24 hour) }\end{array}$ & $\begin{array}{c}\text { Water-level } \\
\text { altitude }\end{array}$ & Date & $\begin{array}{c}\text { Time } \\
\text { (24 hour) }\end{array}$ & $\begin{array}{c}\text { Water-level } \\
\text { altitude }\end{array}$ & Date & $\begin{array}{c}\text { Time } \\
\text { (24 hour) }\end{array}$ & $\begin{array}{c}\text { Water-level } \\
\text { altitude }\end{array}$ \\
\hline \multirow[t]{16}{*}{$7 / 27 / 95$} & 0800 & $1,447.93$ & $7 / 29 / 95$ & 1800 & $1,447.54$ & $8 / 1 / 95$ & 0400 & $1,447.24$ \\
\hline & 0900 & $1,447.93$ & & 1900 & $1,447.53$ & & 0500 & $1,447.23$ \\
\hline & 1000 & $1,447.93$ & & 2000 & $1,447.52$ & & 0600 & $1,447.22$ \\
\hline & 1100 & $1,447.92$ & & 2100 & $1,447.52$ & & 0700 & $1,447.22$ \\
\hline & 1200 & $1,447.91$ & & 2200 & $1,447.49$ & & 0800 & $1,447.22$ \\
\hline & 1300 & $1,447.90$ & & 2300 & $1,447.48$ & & 0900 & $1,447.22$ \\
\hline & 1400 & $1,447.87$ & $7 / 30 / 95$ & 0000 & $1,447.48$ & & 1000 & $1,447.22$ \\
\hline & 1500 & $1,447.87$ & & 0100 & $1,447.47$ & & 1100 & $1,447.23$ \\
\hline & 1600 & $1,447.86$ & & 0200 & $1,447.46$ & & 1200 & $1,447.22$ \\
\hline & 1700 & $1,447.84$ & & 0300 & $1,447.46$ & & 1300 & $1,447.21$ \\
\hline & 1800 & $1,447.85$ & & 0400 & $1,447.45$ & & 1400 & $1,447.20$ \\
\hline & 1900 & $1,447.87$ & & 0500 & $1,447.45$ & & 1500 & $1,447.19$ \\
\hline & 2000 & $1,447.79$ & & 0600 & $1,447.44$ & & 1600 & $1,447.17$ \\
\hline & 2100 & $1,447.82$ & & 0700 & $1,447.42$ & & 1700 & $1,447.16$ \\
\hline & 2200 & $1,447.81$ & & 0800 & $1,447.41$ & & 1800 & $1,447.16$ \\
\hline & 2300 & $1,447.80$ & & 0900 & $1,447.41$ & & 1900 & $1,447.16$ \\
\hline \multirow[t]{24}{*}{$7 / 28 / 95$} & 0000 & $1,447.80$ & & 1000 & $1,447.41$ & & 2000 & $1,447.17$ \\
\hline & 0100 & $1,447.78$ & & 1100 & $1,447.40$ & & 2100 & $1,447.14$ \\
\hline & 0200 & $1,447.78$ & & 1200 & $1,447.39$ & & 2200 & $1,447.16$ \\
\hline & 0300 & $1,447.77$ & & 1300 & $1,447.39$ & & 2300 & $1,447.15$ \\
\hline & 0400 & $1,447.77$ & & 1400 & $1,447.40$ & $8 / 2 / 95$ & 0000 & $1,447.16$ \\
\hline & 0500 & $1,447.76$ & & 1500 & $1,447.42$ & & 0100 & $1,447.16$ \\
\hline & 0600 & $1,447.75$ & & 1600 & $1,447.40$ & & 0200 & $1,447.15$ \\
\hline & 0700 & $1,447.73$ & & 1700 & $1,447.39$ & & 0300 & $1,447.15$ \\
\hline & 0800 & $1,447.73$ & & 1800 & $1,447.40$ & & 0400 & $1,447.15$ \\
\hline & 0900 & $1,447.72$ & & 1900 & $1,447.39$ & & 0500 & $1,447.14$ \\
\hline & 1000 & $1,447.71$ & & 2000 & $1,447.40$ & & 0600 & $1,447.14$ \\
\hline & 1100 & $1,447.71$ & & 2100 & $1,447.38$ & & 0700 & $1,447.14$ \\
\hline & 1200 & $1,447.71$ & & 2200 & $1,447.39$ & & 0800 & $1,447.13$ \\
\hline & 1300 & $1,447.68$ & & 2300 & $1,447.38$ & & 0900 & $1,447.13$ \\
\hline & 1400 & $1,447.68$ & $7 / 31 / 95$ & 0000 & $1,447.39$ & & 1000 & $1,447.12$ \\
\hline & 1500 & $1,447.66$ & & 0100 & $1,447.38$ & & 1100 & $1,447.11$ \\
\hline & 1600 & $1,447.68$ & & 0200 & $1,447.38$ & & 1200 & $1,447.10$ \\
\hline & 1700 & $1,447.67$ & & 0300 & $1,447.38$ & & 1300 & $1,447.10$ \\
\hline & 1800 & $1,447.66$ & & 0400 & $1,447.38$ & & 1400 & $1,447.08$ \\
\hline & 1900 & $1,447.65$ & & 0500 & $1,447.38$ & & 1500 & $1,447.06$ \\
\hline & 2000 & $1,447.64$ & & 0600 & $1,447.38$ & & 1600 & $1,447.05$ \\
\hline & 2100 & $1,447.63$ & & 0700 & $1,447.37$ & & 1700 & $1,447.07$ \\
\hline & 2200 & $1,447.64$ & & 0800 & $1,447.37$ & & 1800 & $1,447.04$ \\
\hline & 2300 & $1,447.64$ & & 0900 & $1,447.37$ & & 1900 & $1,447.05$ \\
\hline \multirow[t]{18}{*}{$7 / 29 / 95$} & 0000 & $1,447.64$ & & 1000 & $1,447.37$ & & 2000 & $1,447.05$ \\
\hline & 0100 & $1,447.63$ & & 1100 & $1,447.36$ & & 2100 & $1,447.04$ \\
\hline & 0200 & $1,447.63$ & & 1200 & $1,447.36$ & & 2200 & $1,447.04$ \\
\hline & 0300 & $1,447.63$ & & 1300 & $1,447.35$ & & 2300 & $1,447.04$ \\
\hline & 0400 & $1,447.63$ & & 1400 & $1,447.35$ & $8 / 3 / 95$ & 0000 & $1,447.03$ \\
\hline & 0500 & $1,447.62$ & & 1500 & $1,447.33$ & & 0100 & $1,447.03$ \\
\hline & 0600 & $1,447.62$ & & 1600 & $1,447.31$ & & 0200 & $1,447.03$ \\
\hline & 0700 & $1,447.61$ & & 1700 & $1,447.29$ & & 0300 & $1,447.03$ \\
\hline & 0800 & $1,447.61$ & & 1800 & $1,447.26$ & & 0400 & $1,447.03$ \\
\hline & 0900 & $1,447.60$ & & 1900 & $1,447.27$ & & 0500 & $1,447.03$ \\
\hline & 1000 & $1,447.59$ & & 2000 & $1,447.27$ & & 0600 & $1,447.03$ \\
\hline & 1100 & $1,447.59$ & & 2100 & $1,447.26$ & & 0700 & $1,447.02$ \\
\hline & 1200 & $1,447.59$ & & 2200 & $1,447.26$ & & 0800 & $1,447.01$ \\
\hline & 1300 & $1,447.58$ & & 2300 & $1,447.26$ & & 0900 & $1,447.01$ \\
\hline & 1400 & $1,447.57$ & $8 / 1 / 95$ & 0000 & $1,447.26$ & & 1000 & $1,447.01$ \\
\hline & 1500 & $1,447.56$ & & 0100 & $1,447.26$ & & 1100 & $1,447.00$ \\
\hline & 1600 & $1,447.55$ & & 0200 & $1,447.25$ & & 1200 & $1,447.00$ \\
\hline & 1700 & $1,447.56$ & & 0300 & $1,447.24$ & & 1300 & $1,447.00$ \\
\hline
\end{tabular}


Table 9. Water-level altitudes for Winchester pond B (west pond), June-September, 1995--Continued

\begin{tabular}{|c|c|c|c|c|c|c|c|c|}
\hline Date & $\begin{array}{c}\text { Time } \\
\text { (24 hour) }\end{array}$ & $\begin{array}{l}\text { Water-level } \\
\text { altitude }\end{array}$ & Date & $\begin{array}{c}\text { Time } \\
\text { (24 hour) }\end{array}$ & $\begin{array}{l}\text { Water-level } \\
\text { altitude }\end{array}$ & Date & $\begin{array}{c}\text { Time } \\
\text { (24 hour) }\end{array}$ & $\begin{array}{c}\text { Water-level } \\
\text { altitude }\end{array}$ \\
\hline \multirow[t]{10}{*}{$8 / 3 / 95$} & 1400 & $1,447.00$ & $8 / 5 / 95$ & 2300 & $1,446.59$ & $8 / 8 / 95$ & 0900 & $1,446.31$ \\
\hline & 1500 & $1,447.02$ & $8 / 6 / 95$ & 0000 & $1,446.59$ & & 1000 & $1,446.31$ \\
\hline & 1600 & $1,447.01$ & & 0100 & $1,446.58$ & & 1100 & $1,446.31$ \\
\hline & 1700 & $1,446.97$ & & 0200 & $1,446.58$ & & 1200 & $1,446.30$ \\
\hline & 1800 & $1,446.97$ & & 0300 & $1,446.57$ & & 1300 & $1,446.29$ \\
\hline & 1900 & $1,446.97$ & & 0400 & $1,446.56$ & & 1400 & $1,446.28$ \\
\hline & 2000 & $1,446.96$ & & 0500 & $1,446.55$ & & 1500 & $1,446.27$ \\
\hline & 2100 & $1,446.95$ & & 0600 & $1,446.54$ & & 1600 & $1,446.29$ \\
\hline & 2200 & $1,446.95$ & & 0700 & $1,446.52$ & & 1700 & $1,446.26$ \\
\hline & 2300 & $1,446.94$ & & 0800 & $1,446.51$ & & 1800 & $1,446.26$ \\
\hline \multirow[t]{25}{*}{$8 / 4 / 95$} & 0000 & $1,446.96$ & & 0900 & $1,446.49$ & & 1900 & $1,446.25$ \\
\hline & 0100 & $1,446.96$ & & 1000 & $1,446.49$ & & 2000 & $1,446.23$ \\
\hline & 0200 & $1,446.96$ & & 1100 & $1,446.48$ & & 2100 & $1,446.21$ \\
\hline & 0300 & $1,446.97$ & & 1200 & $1,446.48$ & & 2200 & $1,446.21$ \\
\hline & 0400 & $1,446.98$ & & 1300 & $1,446.49$ & & 2300 & $1,446.20$ \\
\hline & 0500 & $1,446.98$ & & 1400 & $1,446.48$ & $8 / 9 / 95$ & 0000 & $1,446.19$ \\
\hline & 0600 & $1,446.98$ & & 1500 & $1,446.45$ & & 0100 & $1,446.18$ \\
\hline & 0700 & $1,446.99$ & & 1600 & $1,446.47$ & & 0200 & $1,446.17$ \\
\hline & 0800 & $1,446.99$ & & 1700 & $1,446.45$ & & 0300 & $1,446.16$ \\
\hline & 0900 & $1,446.98$ & & 1800 & $1,446.46$ & & 0400 & $1,446.15$ \\
\hline & 1000 & $1,446.97$ & & 1900 & $1,446.47$ & & 0500 & $1,446.13$ \\
\hline & 1100 & $1,446.96$ & & 2000 & $1,446.49$ & & 0600 & $1,446.12$ \\
\hline & 1200 & $1,446.97$ & & 2100 & $1,446.49$ & & 0700 & $1,446.10$ \\
\hline & 1300 & $1,446.95$ & & 2200 & $1,446.48$ & & 0800 & $1,446.10$ \\
\hline & 1345 & $1,446.92$ & & 2300 & $1,446.48$ & & 0900 & $1,446.09$ \\
\hline & 1430 & $1,446.94$ & $8 / 7 / 95$ & 0000 & $1,446.48$ & & 1000 & $1,446.09$ \\
\hline & 1500 & $1,446.89$ & & 0100 & $1,446.49$ & & 1100 & $1,446.08$ \\
\hline & 1600 & $1,446.90$ & & 0200 & $1,446.49$ & & 1200 & $1,446.07$ \\
\hline & 1700 & $1,446.86$ & & 0300 & $1,446.49$ & & 1300 & $1,446.06$ \\
\hline & 1800 & $1,446.87$ & & 0400 & $1,446.49$ & & 1400 & $1,446.04$ \\
\hline & 1900 & $1,446.84$ & & 0500 & $1,446.49$ & & 1500 & $1,446.05$ \\
\hline & 2000 & $1,446.85$ & & 0600 & $1,446.49$ & & 1600 & $1,446.04$ \\
\hline & 2100 & $1,446.82$ & & 0700 & $1,446.49$ & & 1700 & $1,446.00$ \\
\hline & 2200 & $1,446.82$ & & 0800 & $1,446.48$ & & 1800 & $1,446.03$ \\
\hline & 2300 & $1,446.82$ & & 0900 & $1,446.48$ & & 1900 & $1,446.01$ \\
\hline \multirow[t]{23}{*}{$8 / 5 / 95$} & 0000 & $1,446.81$ & & 1000 & $1,446.48$ & & 2000 & $1,445.98$ \\
\hline & 0100 & $1,446.81$ & & 1100 & $1,446.47$ & & 2100 & $1,445.97$ \\
\hline & 0200 & $1,446.80$ & & 1200 & $1,446.47$ & & 2200 & $1,445.97$ \\
\hline & 0300 & $1,446.80$ & & 1300 & $1,446.45$ & & 2300 & $1,445.97$ \\
\hline & 0400 & $1,446.78$ & & 1400 & $1,446.43$ & $8 / 10 / 95$ & 0000 & $1,445.96$ \\
\hline & 0500 & $1,446.78$ & & 1500 & $1,446.42$ & & 0100 & $1,445.95$ \\
\hline & 0600 & $1,446.77$ & & 1600 & $1,446.41$ & & 0200 & $1,445.95$ \\
\hline & 0700 & $1,446.74$ & & 1700 & $1,446.42$ & & 0300 & $1,445.94$ \\
\hline & 0800 & $1,446.74$ & & 1800 & $1,446.42$ & & 0400 & $1,445.94$ \\
\hline & 0900 & $1,446.73$ & & 1900 & $1,446.40$ & & 0500 & $1,445.93$ \\
\hline & 1000 & $1,446.72$ & & 2000 & $1,446.39$ & & 0600 & $1,445.92$ \\
\hline & 1100 & $1,446.70$ & & 2100 & $1,446.37$ & & 0700 & $1,445.92$ \\
\hline & 1200 & $1,446.69$ & & 2200 & $1,446.37$ & & 0800 & $1,445.91$ \\
\hline & 1300 & $1,446.67$ & & 2300 & $1,446.36$ & & 0900 & $1,445.90$ \\
\hline & 1400 & $1,446.67$ & $8 / 8 / 95$ & 0000 & $1,446.35$ & & 1000 & $1,445.90$ \\
\hline & 1500 & $1,446.65$ & & 0100 & $1,446.35$ & & 1100 & $1,445.90$ \\
\hline & 1600 & $1,446.64$ & & 0200 & $1,446.36$ & & 1200 & $1,445.88$ \\
\hline & 1700 & $1,446.63$ & & 0300 & $1,446.34$ & & 1300 & $1,445.87$ \\
\hline & 1800 & $1,446.62$ & & 0400 & $1,446.34$ & & 1400 & $1,445.86$ \\
\hline & 1900 & $1,446.61$ & & 0500 & $1,446.33$ & & 1500 & $1,445.84$ \\
\hline & 2000 & $1,446.60$ & & 0600 & $1,446.33$ & & 1600 & $1,445.86$ \\
\hline & 2100 & $1,446.59$ & & 0700 & $1,446.32$ & & 1700 & $1,445.82$ \\
\hline & 2200 & $1,446.59$ & & 0800 & $1,446.32$ & & 1800 & $1,445.80$ \\
\hline
\end{tabular}


Table 9. Water-level altitudes for Winchester pond B (west pond), June-September, 1995--Continued

\begin{tabular}{|c|c|c|c|c|c|c|c|c|}
\hline Date & $\begin{array}{c}\text { Time } \\
\text { (24 hour) }\end{array}$ & $\begin{array}{l}\text { Water-level } \\
\text { altitude }\end{array}$ & Date & $\begin{array}{c}\text { Time } \\
\text { (24 hour) }\end{array}$ & $\begin{array}{l}\text { Water-level } \\
\text { altitude }\end{array}$ & Date & $\begin{array}{c}\text { Time } \\
\text { (24 hour) }\end{array}$ & $\begin{array}{l}\text { Water-level } \\
\text { altitude }\end{array}$ \\
\hline \multirow[t]{5}{*}{$8 / 10 / 95$} & 1900 & $1,445.80$ & $8 / 13 / 95$ & 0500 & $1,445.45$ & $8 / 15 / 95$ & 1500 & $1,445.14$ \\
\hline & 2000 & $1,445.79$ & & 0600 & $1,445.44$ & & 1600 & $1,445.11$ \\
\hline & 2100 & $1,445.78$ & & 0700 & $1,445.42$ & & 1700 & $1,445.14$ \\
\hline & 2200 & $1,445.78$ & & 0800 & $1,445.41$ & & 1800 & $1,445.10$ \\
\hline & 2300 & $1,445.78$ & & 0900 & $1,445.41$ & & 1900 & $1,445.09$ \\
\hline \multirow[t]{24}{*}{$8 / 11 / 95$} & 0000 & $1,445.77$ & & 1000 & $1,445.40$ & & 2000 & $1,445.10$ \\
\hline & 0100 & $1,445.77$ & & 1100 & $1,445.40$ & & 2100 & $1,445.07$ \\
\hline & 0200 & $1,445.77$ & & 1200 & $1,445.40$ & & 2200 & $1,445.07$ \\
\hline & 0300 & $1,445.76$ & & 1300 & $1,445.39$ & & 2300 & $1,445.06$ \\
\hline & 0400 & $1,445.75$ & & 1400 & $1,445.40$ & $8 / 16 / 95$ & 0000 & $1,445.05$ \\
\hline & 0500 & $1,445.75$ & & 1500 & $1,445.39$ & & 0100 & $1,445.05$ \\
\hline & 0600 & $1,445.74$ & & 1600 & $1,445.37$ & & 0200 & $1,445.04$ \\
\hline & 0700 & $1,445.73$ & & 1700 & $1,445.39$ & & 0300 & $1,445.04$ \\
\hline & 0800 & $1,445.72$ & & 1800 & $1,445.37$ & & 0400 & $1,445.03$ \\
\hline & 0900 & $1,445.73$ & & 1900 & $1,445.37$ & & 0500 & $1,445.03$ \\
\hline & 1000 & $1,445.72$ & & 2000 & $1,445.40$ & & 0600 & $1,445.02$ \\
\hline & 1100 & $1,445.71$ & & 2100 & $1,445.38$ & & 0700 & $1,445.00$ \\
\hline & 1200 & $1,445.72$ & & 2200 & $1,445.37$ & & 0800 & $1,445.00$ \\
\hline & 1300 & $1,445.71$ & & 2300 & $1,445.37$ & & 0900 & $1,444.99$ \\
\hline & 1400 & $1,445.71$ & $8 / 14 / 95$ & 0000 & $1,445.37$ & & 1000 & $1,444.99$ \\
\hline & 1500 & $1,445.67$ & & 0100 & $1,445.38$ & & 1100 & $1,444.97$ \\
\hline & 1600 & $1,445.67$ & & 0200 & $1,445.37$ & & 1200 & $1,444.96$ \\
\hline & 1700 & $1,445.66$ & & 0300 & $1,445.37$ & & 1300 & $1,444.95$ \\
\hline & 1800 & $1,445.64$ & & 0400 & $1,445.37$ & & 1400 & $1,444.90$ \\
\hline & 1900 & $1,445.66$ & & 0500 & $1,445.37$ & & 1500 & $1,444.94$ \\
\hline & 2000 & $1,445.66$ & & 0600 & $1,445.37$ & & 1600 & $1,444.92$ \\
\hline & 2100 & $1,445.65$ & & 0700 & $1,445.36$ & & 1700 & 1.444 .91 \\
\hline & 2200 & $1,445.65$ & & 0800 & $1,445.35$ & & 1800 & 1.444 .91 \\
\hline & 2300 & $1,445.65$ & & 0900 & $1,445.35$ & & 1900 & $1,444.85$ \\
\hline \multirow[t]{24}{*}{$8 / 12 / 95$} & 0000 & $1,445.64$ & & 1000 & $1,445.35$ & & 2000 & $1,444.89$ \\
\hline & 0100 & $1,445.64$ & & 1100 & $1,445.36$ & & 2100 & $1,444.88$ \\
\hline & 0200 & $1,445.64$ & & 1200 & $1,445.33$ & & 2200 & $1,444.91$ \\
\hline & 0300 & $1,445.63$ & & 1300 & $1,445.34$ & & 2300 & $1,444.89$ \\
\hline & 0400 & $1,445.63$ & & 1400 & $1,445.33$ & $8 / 17 / 95$ & 0000 & $1,444.90$ \\
\hline & 0500 & $1,445.62$ & & 1500 & $1,445.29$ & & 0100 & $1,444.91$ \\
\hline & 0600 & $1,445.61$ & & 1600 & $1,445.29$ & & 0200 & $1,444.91$ \\
\hline & 0700 & $1,445.61$ & & 1700 & $1,445.27$ & & 0300 & $1,444.91$ \\
\hline & 0800 & $1,445.60$ & & 1800 & $1,445.27$ & & 0400 & $1,444.91$ \\
\hline & 0900 & $1,445.60$ & & 1900 & $1,445.25$ & & 0500 & $1,444.91$ \\
\hline & 1000 & $1,445.59$ & & 2000 & $1,445.25$ & & 0600 & $1,444.91$ \\
\hline & 1100 & $1,445.59$ & & 2100 & $1,445.23$ & & 0700 & $1,444.90$ \\
\hline & 1200 & $1,445.60$ & & 2200 & $1,445.24$ & & 0800 & $1,444.91$ \\
\hline & 1300 & $1,445.62$ & & 2300 & $1,445.25$ & & 0900 & $1,444.90$ \\
\hline & 1400 & $1,445.58$ & $8 / 15 / 95$ & 0000 & $1,445.24$ & & 1000 & $1,444.90$ \\
\hline & 1500 & $1,445.58$ & & 0100 & $1,445.23$ & & 1100 & $1,444.90$ \\
\hline & 1600 & $1,445.60$ & & 0200 & $1,445.24$ & & 1200 & $1,444.90$ \\
\hline & 1700 & $1,445.51$ & & 0300 & $1,445.23$ & & 1300 & $1,444.88$ \\
\hline & 1800 & $1,445.51$ & & 0400 & $1,445.23$ & & 1400 & $1,444.86$ \\
\hline & 1900 & $1,445.52$ & & 0500 & $1,445.23$ & & 1500 & $1,444.89$ \\
\hline & 2000 & $1,445.50$ & & 0600 & $1,445.22$ & & 1600 & $1,444.83$ \\
\hline & 2100 & $1,445.50$ & & 0700 & $1,445.21$ & & 1700 & $1,444.83$ \\
\hline & 2200 & $1,445.49$ & & 0800 & $1,445.20$ & & 1800 & $1,444.82$ \\
\hline & 2300 & $1,445.49$ & & 0900 & $1,445.21$ & & 1900 & $1,444.80$ \\
\hline \multirow[t]{5}{*}{$8 / 13 / 95$} & 0000 & $1,445.48$ & & 1000 & $1,445.21$ & & 2000 & $1,444.80$ \\
\hline & 0100 & $1,445.47$ & & 1100 & $1,445.21$ & & 2100 & $1,444.79$ \\
\hline & 0200 & $1,445.46$ & & 1200 & $1,445.21$ & & 2200 & $1,444.79$ \\
\hline & 0300 & $1,445.46$ & & 1300 & $1,445.16$ & & 2300 & $1,444.79$ \\
\hline & 0400 & $1,445.45$ & & 1400 & $1,445.17$ & $8 / 18 / 95$ & 0000 & $1,444.79$ \\
\hline
\end{tabular}


Table 9. Water-level altitudes for Winchester pond B (west pond), June-September, 1995--Continued

\begin{tabular}{|c|c|c|c|c|c|c|c|c|}
\hline Date & $\begin{array}{c}\text { Time } \\
\text { (24 hour) }\end{array}$ & $\begin{array}{c}\text { Water-level } \\
\text { altitude }\end{array}$ & Date & $\begin{array}{c}\text { Time } \\
\text { (24 hour) }\end{array}$ & $\begin{array}{l}\text { Water-level } \\
\text { altitude }\end{array}$ & Date & $\begin{array}{c}\text { Time } \\
\text { (24 hour) }\end{array}$ & $\begin{array}{c}\text { Water-level } \\
\text { altitude }\end{array}$ \\
\hline \multirow[t]{23}{*}{ 8/18/95 } & 0100 & $1,444.78$ & $8 / 20 / 95$ & 1100 & $1,444.12$ & $8 / 22 / 95$ & 2100 & $1,443.55$ \\
\hline & 0200 & $1,444.77$ & & 1200 & $1,444.10$ & & 2200 & $1,443.54$ \\
\hline & 0300 & $1,444.76$ & & 1300 & $1,444.10$ & & 2300 & $1,443.51$ \\
\hline & 0400 & $1,444.75$ & & 1400 & $1,444.09$ & $8 / 23 / 95$ & 0000 & $1,443.51$ \\
\hline & 0500 & $1,444.74$ & & 1500 & $1,444.11$ & & 0100 & $1,443.50$ \\
\hline & 0600 & $1,444.74$ & & 1600 & $1,444.11$ & & 0200 & $1,443.49$ \\
\hline & 0700 & $1,444.73$ & & 1700 & $1,444.11$ & & 0300 & $1,443.48$ \\
\hline & 0800 & $1,444.73$ & & 1800 & $1,444.10$ & & 0400 & $1,443.45$ \\
\hline & 0900 & $1,444.72$ & & 1900 & $1,444.08$ & & 0500 & $1,443.44$ \\
\hline & 1000 & $1,444.71$ & & 2000 & $1,444.09$ & & 0600 & $1,443.43$ \\
\hline & 1100 & $1,444.70$ & & 2100 & $1,444.08$ & & 0700 & $1,443.40$ \\
\hline & 1200 & $1,444.68$ & & 2200 & $1,444.08$ & & 0800 & $1,443.39$ \\
\hline & 1300 & $1,444.67$ & & 2300 & $1,444.08$ & & 0900 & $1,443.38$ \\
\hline & 1400 & $1,444.66$ & $8 / 21 / 95$ & 0000 & $1,444.07$ & & 1000 & $1,443.36$ \\
\hline & 1500 & $1,444.63$ & & 0100 & $1,444.07$ & & 1100 & $1,443.36$ \\
\hline & 1600 & $1,444.61$ & & 0200 & $1,444.07$ & & 1200 & $1,443.31$ \\
\hline & 1700 & $1,444.62$ & & 0300 & $1,444.07$ & & 1300 & $1,443.33$ \\
\hline & 1800 & $1,444.58$ & & 0400 & $1,444.07$ & & 1400 & $1,443.30$ \\
\hline & 1900 & $1,444.55$ & & 0500 & $1,444.07$ & & 1500 & $1,443.28$ \\
\hline & 2000 & $1,444.56$ & & 0600 & $1,444.06$ & & 1600 & $1,443.27$ \\
\hline & 2100 & $1,444.53$ & & 0700 & $1,444.06$ & & 1700 & $1,443.26$ \\
\hline & 2200 & $1,444.53$ & & 0800 & $1,444.05$ & & 1800 & $1,443.24$ \\
\hline & 2300 & $1,444.53$ & & 0900 & $1,444.05$ & & 1900 & $1,443.19$ \\
\hline \multirow[t]{24}{*}{$8 / 19 / 95$} & 0000 & $1,444.51$ & & 1000 & $1,444.05$ & & 2000 & $1,443.18$ \\
\hline & 0100 & $1,444.51$ & & 1100 & $1,444.04$ & & 2100 & $1,443.16$ \\
\hline & 0200 & $1,444.49$ & & 1200 & $1,444.03$ & & 2200 & $1,443.15$ \\
\hline & 0300 & $1,444.48$ & & 1300 & $1,444.03$ & & 2300 & $1,443.13$ \\
\hline & 0400 & $1,444.48$ & & 1400 & $1,443.99$ & $8 / 24 / 95$ & 0000 & $1,443.11$ \\
\hline & 0500 & $1,444.46$ & & 1500 & $1,443.99$ & & 0100 & $1,443.10$ \\
\hline & 0600 & $1,444.45$ & & 1600 & $1,443.96$ & & 0200 & $1,443.09$ \\
\hline & 0700 & $1,444.43$ & & 1700 & $1,443.96$ & & 0300 & $1,443.07$ \\
\hline & 0800 & $1,444.43$ & & 1800 & $1,443.94$ & & 0400 & $1,443.05$ \\
\hline & 0900 & $1,444.42$ & & 1900 & $1,443.91$ & & 0500 & $1,443.04$ \\
\hline & 1000 & $1,444.42$ & & 2000 & $1,443.89$ & & 0600 & $1,443.03$ \\
\hline & 1100 & $1,444.40$ & & 2100 & $1,443.88$ & & 0700 & $1,443.00$ \\
\hline & 1200 & $1,444.39$ & & 2200 & $1,443.87$ & & 0800 & $1,442.99$ \\
\hline & 1300 & $1,444.39$ & & 2300 & $1,443.86$ & & 0900 & $1,442.97$ \\
\hline & 1400 & $1,444.37$ & $8 / 22 / 95$ & 0000 & $1,443.85$ & & 1000 & $1,442.96$ \\
\hline & 1500 & $1,444.31$ & & 0100 & $1,443.84$ & & 1100 & $1,442.96$ \\
\hline & 1600 & $1,444.36$ & & 0200 & $1,443.82$ & & 1200 & $1,442.94$ \\
\hline & 1700 & $1,444.33$ & & 0300 & $1,443.81$ & & 1300 & $1,442.92$ \\
\hline & 1800 & $1,444.32$ & & 0400 & $1,443.80$ & & 1400 & $1,442.87$ \\
\hline & 1900 & $1,444.29$ & & 0500 & $1,443.79$ & & 1500 & $1,442.85$ \\
\hline & 2000 & $1,444.29$ & & 0600 & $1,443.77$ & & 1600 & $1,442.84$ \\
\hline & 2100 & $1,444.28$ & & 0700 & $1,443.75$ & & 1700 & $1,442.81$ \\
\hline & 2200 & $1,444.27$ & & 0800 & $1,443.75$ & & 1800 & $1,442.83$ \\
\hline & 2300 & $1,444.25$ & & 0900 & $1,443.74$ & & 1900 & $1,442.80$ \\
\hline \multirow[t]{11}{*}{$8 / 20 / 95$} & 0000 & $1,444.24$ & & 1000 & $1,443.73$ & & 2000 & $1,442.78$ \\
\hline & 0100 & $1,444.23$ & & 1100 & $1,443.71$ & 8/30/95 & 1315 & $1,440.63$ \\
\hline & 0200 & $1,444.22$ & & 1200 & $1,443.70$ & & 1400 & $1,440.57$ \\
\hline & 0300 & $1,444.21$ & & 1300 & $1,443.70$ & & 1500 & $1,440.54$ \\
\hline & 0400 & $1,444.19$ & & 1400 & $1,443.68$ & & 1600 & $1,440.53$ \\
\hline & 0500 & $1,444.18$ & & 1500 & $1,443.63$ & & 1700 & $1,440.54$ \\
\hline & 0600 & $1,444.17$ & & 1600 & $1,443.70$ & & 1800 & $1,440.48$ \\
\hline & 0700 & $1,444.15$ & & 1700 & $1,443.62$ & & 1900 & $1,440.49$ \\
\hline & 0800 & $1,444.15$ & & 1800 & $1,443.58$ & & 2000 & $1,440.45$ \\
\hline & 0900 & $1,444.13$ & & 1900 & $1,443.57$ & & 2100 & $1,440.43$ \\
\hline & 1000 & $1,444.12$ & & 2000 & $1,443.56$ & & 2200 & $1,440.42$ \\
\hline
\end{tabular}


Table 9. Water-level altitudes for Winchester pond B (west pond), June-September, 1995--Continued

\begin{tabular}{|c|c|c|c|c|c|c|c|c|}
\hline Date & $\begin{array}{c}\text { Time } \\
\text { (24 hour) }\end{array}$ & $\begin{array}{c}\text { Water-level } \\
\text { altitude }\end{array}$ & Date & $\begin{array}{c}\text { Time } \\
\text { (24 hour) }\end{array}$ & $\begin{array}{l}\text { Water-level } \\
\text { altitude }\end{array}$ & Date & $\begin{array}{c}\text { Time } \\
\text { (24 hour) }\end{array}$ & $\begin{array}{c}\text { Water-leve } \\
\text { altitude }\end{array}$ \\
\hline $8 / 30 / 95$ & 2300 & $1,440.40$ & $9 / 2 / 95$ & 0400 & $1,439.50$ & 9/4/95 & 1000 & $1,439.29$ \\
\hline \multirow[t]{24}{*}{$8 / 31 / 95$} & 0000 & $1,440.39$ & & 0500 & $1,439.48$ & & 1100 & $1,439.32$ \\
\hline & 0100 & $1,440.38$ & & 0600 & $1,439.45$ & & 1200 & $1,439.31$ \\
\hline & 0200 & $1,440.36$ & & 0700 & $1,439.43$ & & 1300 & $1,439.30$ \\
\hline & 0300 & $1,440.35$ & & 0800 & $1,439.40$ & & 1400 & $1,439.30$ \\
\hline & 0400 & $1,440.34$ & & 0900 & $1,439.37$ & & 1500 & $1,439.29$ \\
\hline & 0500 & $1,440.33$ & & 1000 & $1,439.36$ & & 1600 & $1,439.31$ \\
\hline & 0600 & $1,440.32$ & & 1100 & $1,439.37$ & & 1700 & $1,439.31$ \\
\hline & 0700 & $1,440.30$ & & 1200 & $1,439.36$ & & 1800 & $1,439.30$ \\
\hline & 0800 & $1,440.28$ & & 1300 & $1,439.36$ & & 1900 & $1,439.33$ \\
\hline & 0900 & $1,440.27$ & & 1400 & $1,439.32$ & & 2000 & $1,439.30$ \\
\hline & 1000 & $1,440.24$ & & 1600 & $1,439.30$ & & 2100 & $1,439.30$ \\
\hline & 1100 & $1,440.22$ & & 1700 & $1,439.30$ & & 2200 & $1,439.30$ \\
\hline & 1200 & $1,440.19$ & & 1800 & $1,439.31$ & & 2300 & $1,439.30$ \\
\hline & 1300 & $1,440.18$ & & 1900 & $1,439.32$ & 9/5/95 & 0000 & $1,439.30$ \\
\hline & 1400 & $1,440.16$ & & 2000 & $1,439.31$ & & 0100 & $1,439.30$ \\
\hline & 1500 & $1,440.12$ & & 2100 & $1,439.30$ & & 0200 & $1,439.30$ \\
\hline & 1600 & $1,440.13$ & & 2200 & $1,439.31$ & & 0300 & $1,439.30$ \\
\hline & 1700 & $1,440.11$ & & 2300 & $1,439.31$ & & 0400 & $1,439.29$ \\
\hline & 1800 & $1,440.09$ & $9 / 3 / 95$ & 0000 & $1,439.31$ & & 0500 & $1,439.29$ \\
\hline & 1900 & $1,440.05$ & & 0100 & $1,439.31$ & & 0600 & $1,439.29$ \\
\hline & 2000 & $1,440.04$ & & 0200 & $1,439.30$ & & 0700 & $1,439.28$ \\
\hline & 2100 & $1,440.02$ & & 0300 & $1,439.31$ & & 0800 & $1,439.28$ \\
\hline & 2200 & $1,440.00$ & & 0400 & $1,439.30$ & & 0900 & $1,439.29$ \\
\hline & 2300 & $1,440.00$ & & 0500 & $1,439.30$ & & 1000 & $1,439.28$ \\
\hline \multirow[t]{24}{*}{$9 / 1 / 95$} & 0000 & $1,439.98$ & & 0600 & $1,439.30$ & & 1100 & $1,439.27$ \\
\hline & 0100 & $1,439.96$ & & 0700 & $1,439.30$ & & 1200 & $1,439.25$ \\
\hline & 0200 & $1,439.95$ & & 0800 & $1,439.29$ & & 1300 & $1,439.22$ \\
\hline & 0300 & $1,439.94$ & & 0900 & $1,439.30$ & & 1400 & $1,439.23$ \\
\hline & 0400 & $1,439.92$ & & 1000 & $1,439.30$ & & 1500 & $1,439.20$ \\
\hline & 0500 & $1,439.91$ & & 1100 & $1,439.30$ & & 1600 & $1,439.20$ \\
\hline & 0600 & $1,439.90$ & & 1200 & $1,439.30$ & & 1700 & $1,439.21$ \\
\hline & 0700 & $1,439.87$ & & 1300 & $1,439.30$ & & 1800 & $1,439.17$ \\
\hline & 0800 & $1,439.86$ & & 1400 & $1,439.31$ & & 1900 & $1,439.15$ \\
\hline & 0900 & $1,439.83$ & & 1500 & $1,439.31$ & & 2000 & $1,439.14$ \\
\hline & 1000 & $1,439.81$ & & 1600 & $1,439.32$ & & 2100 & $1,439.13$ \\
\hline & 1100 & $1,439.81$ & & 1700 & $1,439.33$ & & 2200 & $1,439.13$ \\
\hline & 1200 & $1,439.77$ & & 1800 & $1,439.33$ & & 2300 & $1,439.12$ \\
\hline & 1300 & $1,439.75$ & & 1900 & $1,439.32$ & $9 / 6 / 95$ & 0000 & $1,439.11$ \\
\hline & 1400 & $1,439.78$ & & 2000 & $1,439.31$ & & 0100 & $1,439.11$ \\
\hline & 1500 & $1,439.74$ & & 2100 & $1,439.31$ & & 0200 & $1,439.09$ \\
\hline & 1600 & $1,439.70$ & & 2200 & $1,439.31$ & & 0300 & $1,439.08$ \\
\hline & 1700 & $1,439.68$ & & 2300 & $1,439.30$ & & 0400 & $1,439.08$ \\
\hline & 1800 & $1,439.65$ & $9 / 4 / 95$ & 0000 & $1,439.30$ & & 0500 & $1,439.07$ \\
\hline & 1900 & $1,439.63$ & & 0100 & $1,439.30$ & & 0600 & $1,439.05$ \\
\hline & 2000 & $1,439.62$ & & 0200 & $1,439.30$ & & 0700 & $1,439.03$ \\
\hline & 2100 & $1,439.61$ & & 0300 & $1,439.30$ & & 0800 & $1,439.03$ \\
\hline & 2200 & $1,439.60$ & & 0400 & $1,439.30$ & & 0900 & $1,439.05$ \\
\hline & 2300 & $1,439.58$ & & 0500 & $1,439.29$ & & 1000 & $1,439.05$ \\
\hline \multirow[t]{4}{*}{$9 / 2 / 95$} & 0000 & $1,439.56$ & & 0600 & $1,439.30$ & & 1100 & $1,439.06$ \\
\hline & 0100 & $1,439.55$ & & 0700 & $1,439.28$ & $\cdot$ & 1130 & 1.439 .03 \\
\hline & 0200 & $1,439.54$ & & 0800 & $1,439.29$ & & & \\
\hline & 0300 & $1,439.53$ & & 0930 & $1,439.30$ & & & \\
\hline
\end{tabular}

